\title{
One star, two star, red star, blue star: an updated planetary nebula central star distance catalogue from Gaia EDR3
}

\author{
N. Chornay and N. A. Walton
}

Institute of Astronomy, University of Cambridge, Madingley Road, Cambridge, CB3 0HA, United Kingdom e-mail: njc89@ast.cam.ac.uk, e-mail: naw@ast.cam.ac.uk

September 2021

\begin{abstract}
Context. Planetary nebulae (PNe) are a brief but important phase of stellar evolution. The study of Galactic PNe has historically been hampered by uncertain distances, but the parallaxes of PN central stars (CSPNe) measured by Gaia are improving the situation. Aims. Gaia's Early Data Release 3 (EDR3) offers higher astrometric precision and greater completeness compared to previous releases. Taking advantage of these improvements requires that the CSPNe in the catalogue be accurately identified.

Methods. We applied our automated technique based on the likelihood ratio method to cross-match known PNe with sources in Gaia EDR3, using an empirically derived position and colour distribution to score candidate matches.

Results. We present a catalogue of over 2000 sources in Gaia EDR3 that our method has identified as likely CSPNe or compact nebula detections. We show how the more precise parallaxes of these sources compare to previous PN statistical distances and introduce an approach to combining them to produce tighter distance constraints. We also discuss Gaia's handling of close companions and bright nebulae.

Conclusions. Gaia is unlocking new avenues for the study of PNe. The catalogue presented here will remain valid for the upcoming Gaia Data Release 3 (DR3) and thus provide a valuable resource for years to come.
\end{abstract}

Key words. planetary nebulae: general - parallaxes - Methods: statistical

\section{Introduction}

Planetary nebulae (PNe) are a brief but enigmatic stage in the evolution of low- and intermediate-mass stars towards the ends of their lives. Central stars of $\mathrm{PNe}(\mathrm{CSPNe})$ are some of the rarest objects observed by Gaia (Gaia Collaboration et al. 2016), a European Space Agency (ESA) mission conducting precision astrometry and photometry for nearly two billion sources in the Milky Way and beyond. Gaia shows great potential for the study of PNe: parallaxes inform distance estimates and in turn CSPN absolute magnitudes and evolutionary phase; proper motions add more dimensions to dynamics and relate PNe to their parent stellar populations, constraining ages and masses; and repeated photometric observations enable the study of variability and binarity.

Unlocking Gaia's potential requires a complete and accurate cross-match of Gaia sources with CSPNe. Chornay \& Walton (2020, hereinafter CW20) used an automated approach to generate an extensive catalogue of candidate CSPNe in Gaia Data Release 2 (DR2, Gaia Collaboration et al. 2018). The nature of Gaia's iterative release process necessitates updates to catalogues based on Gaia data, as the set of sources and their identifications changes between releases (Torra et al. 2021). Moreover, when the Gaia data is itself used for cross-matching, better data translates into improved accuracy. In this work we present a new catalogue of candidate CSPNe and compact PNe in Gaia Early Data Release 3 (EDR3, Gaia Collaboration et al.2021) based on the same methodology as CW20. With a completely new Gaia catalogue not expected for some time, this will provide a valuable resource for the study of Galactic PNe for years to come.

\section{Methods}

\subsection{Input catalogues}

Our PN positions come from the Hong Kong/AAO/Strasbourg $\mathrm{H} \alpha$ (HASH) PN catalogue (Parker et al. 2016, as of 5 July 2021), which now contains 2670 "true" PNe (those with spectroscopic confirmation; an $8 \%$ increase since CW20) and $3800 \mathrm{PNe}$ in total (including likely and possible identifications). We saw in CW20 that three very extended PNe had CSPNe more than $60^{\prime \prime}$ away from the HASH positions, outside of our search radius. To address this we adopt positions for Sh 2-188 from Weidmann \& Gamen (2011) and for FP J1824-0319 and FP J0905-3033 from Parker et al. (2006). Gaia EDR3 is likewise larger than DR2. The longer survey duration (12 more months, for 34 months in total), along with improved calibration, results in higher completeness (7\% more sources overall) and greater astrometric precision. The set of sources in EDR3 is distinct from that of DR2 not only in that more sources are present; some may have disappeared or changed identifiers (Torra et al.|2021). Gaia Data Release 3 (DR3) will include the same sources as EDR3 but with additional data products.

\subsection{Cross-matching}

We use the same method employed in CW20, and here provide a brief review, pointing the reader to that work for a more detailed background. The aim is to automatically match CSPNe or compact/stellar-like nebulae to Gaia sources. The expectation is that these sources will be close to the catalogued position of the PN, but the possibility of non-detections, background inter- 
lopers, and positional inaccuracies necessitates a more nuanced approach than simple nearest-neighbour matching.

The likelihood ratio method of Sutherland \& Saunders (1992) is well-suited to this aim. Given a candidate match at angular separation $r$, the approach compares the probability of finding a genuine match at $r$ to finding a background object. Our implementation assumes a uniform background density in each field, and a radially symmetric positional distribution of genuine CSPNe parameterised by the radius of the PN (allowing larger $\mathrm{PNe}$ to have more offset CSPNe, albeit with lower probability). We also employ the colour distributions of true CSPNe and background sources, parameterised based on the photometric excess factor (Evans et al. 2018), with the effect of down-weighting colours for sources whose colour is potentially contaminated by nearby sources or background. Distributions are derived iteratively, using sources with secure identifications based on colour to empirically determine the positional distribution of genuine CSPNe, and in turn using the positionally secure sources to update the colour distribution. This works because CSPNe are often much bluer than typical Gaia sources and therefore can be selected purely based on colour with relatively high confidence.

We made a few small changes to the algorithm's treatment of the $G_{\mathrm{BP}}-G_{\mathrm{RP}}$ colours. In deriving the initial and final colour distributions, we only considered sources with $G$ brighter than 19 , which have relatively precise colours. Then in computing the likelihood ratios for individual sources we convolved the densities with the photometric uncertainties, after accounting for the smoothing already applied by the kernel density estimation. This lessens the impact of faint sources with extreme and likely spurious colours. We also adopted the prescription of Riello et al. (2021) for describing a locus of photometric excess values of well-behaved sources. The photometric excess is no longer used by Gaia as a filter for published photometry, though for large excess values the photometry is still not very discriminative, as it likely dominated by the background.

We retrieved all Gaia EDR3 sources within a 60" radius of the PN positions from HASH. All of these were used in calculating the background density and colour distribution (the latter only for $G<19$ ), while only the subset of sources within half each PN angular size (taken from HASH) plus $2^{\prime \prime}$ were considered as candidates. For each PN, the set of candidate likelihood ratios were used to calculate reliabilities (the probability that a given candidate is the correct match).

\section{Catalogue}

The improved method applied to EDR3 produces a bimodal distribution of top match reliabilities similar to that from CW20 for Gaia DR2 (Fig 1. lower left; for clarity only "true" PNe are shown). Most sources have reliabilities close to their values from CW20; these are near the diagonal (region C) in upper left of Fig. 11 Points in regions A and E represent the most significant changes, typically due to removed or new sources respectively. Counts of PNe in these regions are in Table 1, including "likely" and "possible" PNe. In addition there are 207 new PNe in HASH since CW20 (included in Fig. 1, lower left); of these 107 have candidates with reliability $>0.2$. There are also 22 objects published in CW20 that are no longer listed as PNe in HASH (not shown).

As with the previous iteration, the method retrieves blue CSPNe even out to large separations (Fig. 1, right; the largest $\mathrm{PN}$ in the cluster at the upper left has an angular radius of nearly $\left.600^{\prime \prime}\right)$. The few sources with unphysically blue colours are scored lower due to their large photometric uncertainties.
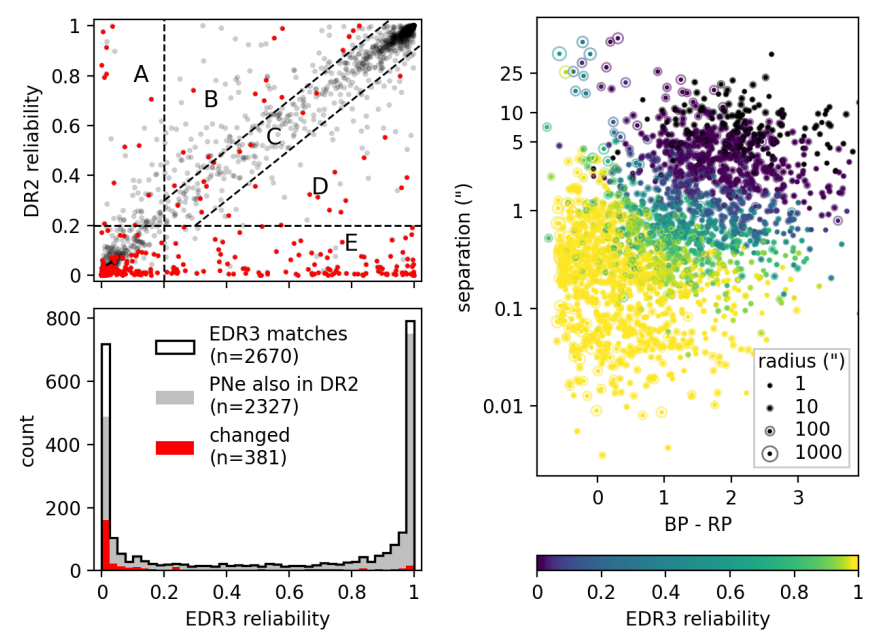

Fig. 1: Matching results for "true" PNe. The top left panel shows the reliability of the CW20 Gaia DR2 matches versus the EDR3 matches from this work for PNe present in both. Red points are $\mathrm{PNe}$ where the location of the best candidate match has changed by more than $00^{\prime \prime} 1$. All of these points are contained in the histogram in the lower left panel, which also includes PNe that were not in CW20. The upper right panel shows the separation versus colour distribution of highest-ranked candidates, colour-coded by reliability with the non-linear scale shown at the bottom right. Rings around markers indicate PN angular sizes.

Table 1: Counts of PNe occupying different regions of the scatter plot in the upper left corner of Fig. 1, representing different changes in reliability relative to the CW20 Gaia DR2 matches.

\begin{tabular}{lllllll}
\hline \hline PN status & match location & \multicolumn{5}{c}{ region } \\
& & $\mathrm{A}$ & $\mathrm{B}$ & $\mathrm{C}$ & $\mathrm{D}$ & $\mathrm{E}$ \\
\hline \multirow{2}{*}{ true } & same & 53 & 105 & 1238 & 47 & 15 \\
\multirow{3}{*}{ other } & changed & 16 & 14 & 10 & 14 & 157 \\
& same & 36 & 58 & 303 & 7 & 3 \\
& changed & 3 & 3 & 2 & 4 & 30 \\
\hline
\end{tabular}

The complete catalogue of the 2117 highest ranked candidates with reliability $>0.2$ is in Table A.1. The catalogue includes the Gaia source identifier corresponding to the best match for each PN, the reliability of that match, and its angular separation from the PN position from HASH. Copied into the catalogue are relevant data from HASH (name, PN G identifier, PN coordinates, angular size, and confirmation status) and Gaia EDR3 (source coordinates, photometry, and astrometry). We additionally include the image parameter determination (IPD, Lindegren et al.2021b) goodness-of-fit harmonic amplitude from Gaia (see Sect. 3.3 and combined statistical and parallax distance derived in this work (see Sect. 4.2).

\subsection{Individual objects}

Fig. 2 shows wide- and narrow-band imagery from the VPHAS+ survey (Drew et al.|2014) overlayed with Gaia EDR3 (and DR2) sources for four PNe chosen to exemplify significant changes encountered in Gaia EDR3. Data from VPHAS+ were not used as part of the matching, but the images, which capture stellar colours and nebula emission, provide good examples of some of the changes encountered in the updated Gaia data. 


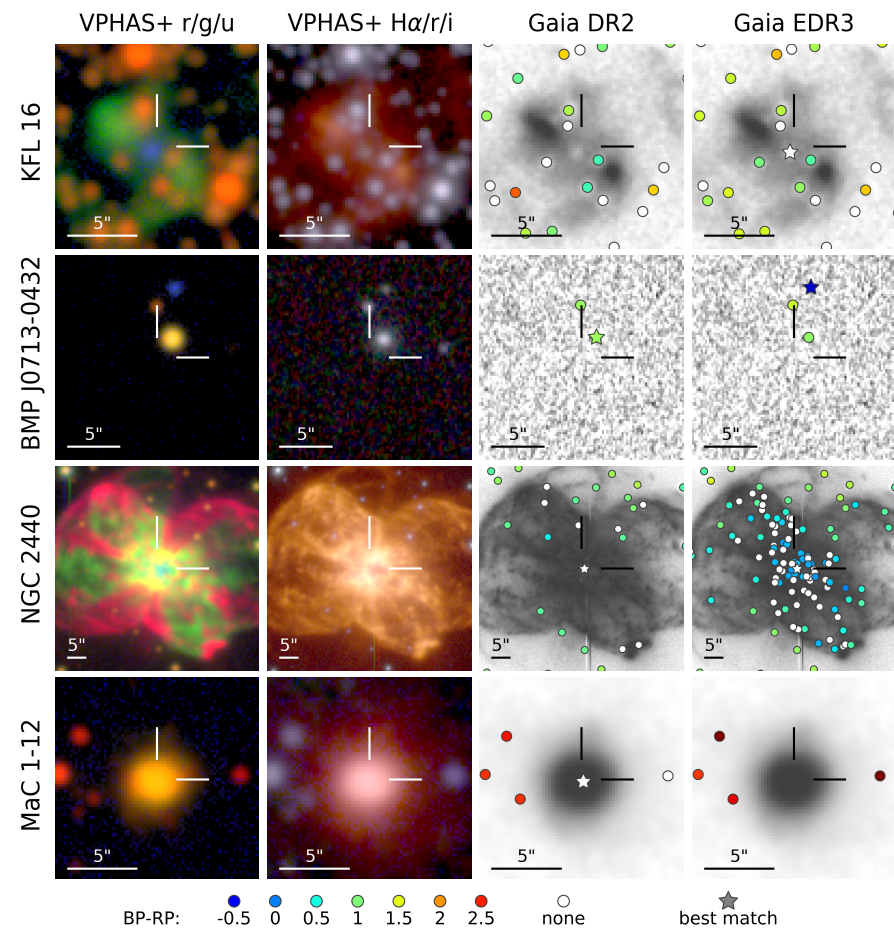

Fig. 2: VPHAS+ images of select PNe centred on their coordinates from HASH. North is up and east is to the left. The left two columns are colour images in different sets of filters, while the right two columns are quotient $\left(r^{\prime}-\mathrm{H} \alpha\right)$ images overlayed with Gaia sources coloured according to their $G_{\mathrm{BP}}-G_{\mathrm{RP}}$ colours, with the best match highlighted.

KFL 16 and BMP J0713-0432 both have new detections in EDR3. The CSPN of KFL 16 has no Gaia colour but is sufficiently central that it is selected; its blue colour is evident in the VPHAS + imagery. The CSPN of BMP J0713-0432 does exhibit a blue $G_{\mathrm{BP}}-G_{\mathrm{RP}}$ colour and as such is correctly identified despite not being the closest source to the PN position from HASH.

NGC 2440 had a clear CSPN detection in DR2. In EDR3 the central region of the PN now contains many nebula detections. The true CSPN is still selected based on its position (it is overwhelmed by the bright nebula in VPHAS + but clear in spacebased imagery), but with reduced confidence on account of the many new nearby sources. In the case of $\mathrm{MaC} 1-12$, the lone detection from DR2 has disappeared, and no matching EDR3 source is found.

\subsection{Visual binaries}

Visual binaries are astrophysically interesting because, among other reasons, characterisation of the companion can provide an estimate of the distance to the pair and thus to the PN. However close companions - whether genuine or merely a chance alignment - can provide a challenge for our matching. Of the $53 \mathrm{PNe}$ with two potential matches (two candidates with reliability $>0.2$ ), about half are pairs with separation less than $1^{\prime \prime}$. These likely include some genuine binary systems, though their blended colour photometry and degraded astrometry makes it difficult to characterise the companion star and secure its relation through astrometry (e.g. González-Santamaría et al.|2020).

Some CSPNe are visual binaries in Hubble Space Telescope (HST) imagery: ten are presented in Ciardullo et al. (1999) ("probable associations") as well as additional pairs in Benetti
Table 2: Gaia detections of known close visual binaries.

\begin{tabular}{lllllll}
\hline \hline PN & $\begin{array}{l}\text { Sep. } \\
\left({ }^{a}\right)\end{array}$ & $\begin{array}{l}\Delta V^{b} \\
(\mathrm{mag})\end{array}$ & Rel. $^{c}$ & $\begin{array}{l}G \\
(\mathrm{mag})\end{array}$ & $\begin{array}{l}\text { Mul. }^{d} \\
(\%)\end{array}$ & RUWE \\
\hline Abell 31 & 0.26 & $>6.8$ & 1.00 & 15.5 & 0 & 1.03 \\
Abell 33 & $1.80^{e}$ & 1.0 & 0.93 & 15.9 & 0 & 1.09 \\
& & & 0.07 & 16.7 & 0 & 0.98 \\
EGB 6 & 0.17 & $\ldots$ & 0.96 & 16.0 & 0 & 2.09 \\
K 1-14 & 0.36 & 2.4 & 1.00 & 16.1 & 70 & 2.41 \\
K 1-22 & $0.35^{e}$ & 0.3 & 0.83 & 16.7 & 88 & 3.47 \\
& & & 0.17 & 16.7 & 79 & 2.94 \\
K 1-27 & 0.56 & 5.1 & 1.00 & 16.0 & 0 & 0.90 \\
Mz 2 & 0.28 & -1.3 & 0.98 & 16.8 & 0 & 0.97 \\
NGC 1535 & 1.04 & 5.5 & 1.00 & 12.1 & 17 & 1.01 \\
NGC 3132 & $1.70^{e}$ & -5.7 & 0.81 & 10.0 & 0 & 1.84 \\
& & & 0.19 & 16.1 & 16 & 2.07 \\
NGC 6818 & 0.09 & 0.7 & 1.00 & 16.3 & 0 & 3.80 \\
NGC 7008 & 0.42 & -1.5 & 1.00 & 13.7 & 93 & 6.07 \\
Sp 3 & 0.31 & -3.7 & 1.00 & 13.1 & 45 & 3.02 \\
\hline
\end{tabular}

Notes. Rows with first columns blank indicate the Gaia-detected companion of the (assumed PN progenitor) in the row above. Single rows indicate that Gaia has not resolved the pair. ${ }^{(a)}$ Literature separation, except where noted. Most values are from Ciardullo et al. (1999), except for EGB 6 (Liebert et al. 2013) and NGC 6818 (Benetti et al. 2003). (b) Difference in HST $V$ magnitude between ionising star and companion (a positive difference indicates companion is fainter). ${ }^{(c)}$ Reliability. (d) IPD multipeak fraction. ${ }^{(e)}$ Gaia value, within 0 ".02 of literature HST value. ${ }^{(f)}$ The ionising star of NGC 3132 is thought to be the fainter of the pair, so highest reliability match here is not correct in that sense.

et al. (2003) and Liebert et al. (2013). Typical separations of known pairs are fractions of an arcsecond; these appear blended from the ground. With the improved resolution of Gaia EDR3, we expect that more of these will be resolved: the source separation limit has decreased to $0{ }^{\prime \prime} .18$ from $00^{\prime \prime} .4$ in DR2. however incompleteness starts to set in below $1^{\prime \prime} .5$ and is quite severe below 0'.7 (Fabricius et al. 2021).

The detections for these PNe are listed in Table 2 Even sources that not separated by Gaia can show signs of binarity if they have a nonzero IPD multipeak fraction, which indicates that some detections show multiple peaks. These can indicate a close visual binary, though depending on the separation it may only be resolved in some scan directions.

Abell 33 and NGC 3132 have companions at wide separations detectable from the ground (though the catalogue position of NGC 3132 is actually closer to that of the companion star, leading to ambiguity in our matching results). Of the closer pairs, only K 1-22 is resolved into separate sources by Gaia, possibly on account of their similar magnitudes. Another four PNe have a notable fraction of multi-peak detections, while the remaining PNe seem to have companions that are either too faint (e.g. Abell 31 and K 1-27) or too close (e.g. NGC 6818). The re-normalised unit weight error (RUWE, indicative of excess astrometric error; Lindegren 2018) values range from normal to significant, and none are labelled as duplicated sources.

\subsection{Nebula detections}

It is expected that for compact PNe Gaia may not detect the CSPN but rather the bright central region of the nebula. Gaia can also detect features in extended PNe; this was seen early on 


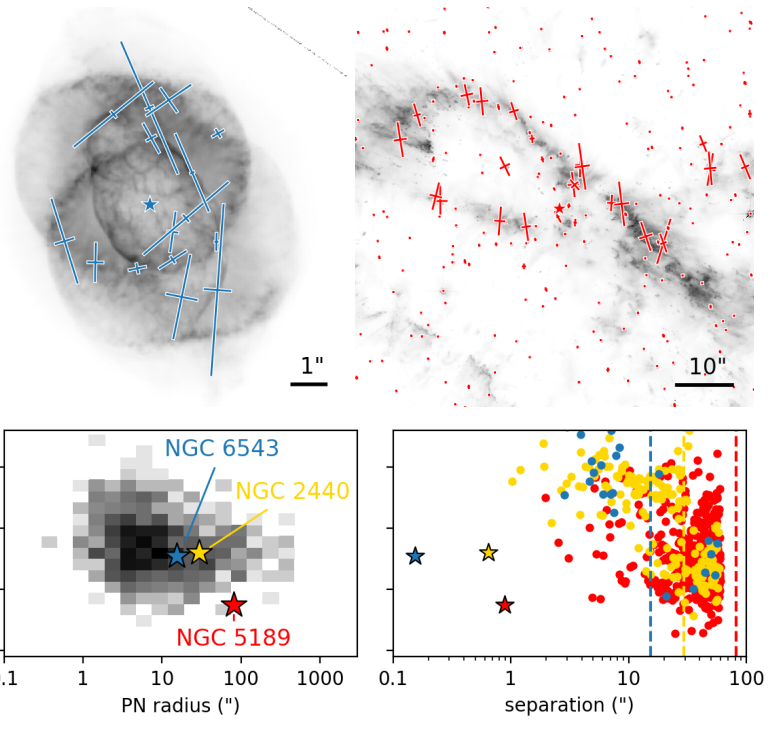

Fig. 3: Nebula detections for bright PNe. The upper images are HST narrowband images of NGC 6543 (left) and the central region of NGC 5189 (right) overlayed with Gaia EDR3 sources (coloured points and crosses). Error bars have been rotated to reflect the direction of the principle component of the error covariance matrices, and scaled by a factor of 100 for visibility. The lower plots show the distribution of IPD goodness-of-fit harmonic amplitudes. The left plot shows the distribution for the best matches versus PN angular radius on a linear colourmap for all best matches in our catalogue, with locations of the CSPNe of the PNe above (and NGC 2440 from Fig. 2) highlighted. The right plot shows all sources in the vicinity of these $\mathrm{PNe}$, plotted against angular separation on a log scale. Vertical lines indicate the PN radii; the lack of points past $60^{\prime \prime}$ is a result of our selection cutoff. (Images credit: Hubble Legacy Archive/ESA/NASA.)

in Gaia's mission for NGC 6543 (Fabricius et al. 2016) 1 1 These detections were filtered out in the first two data releases, leaving only the 11th magnitude CSPN. Now in EDR3 some have returned (Fig. 3, upper left; c.f. Fig. 14 in Fabricius et al. 2016).

A strong indicator that these sources are non-stellar is the large values of IPD goodness-of-fit harmonic amplitude (which we abbreviate to HA). This is newly included in Gaia EDR3, and measures how the astrometric goodness-of-fit varies with scan angle. A large HA suggests an elongated source, such as a galaxy or a partially resolved binary ${ }^{2}$

The lower left panel of Fig. 3 shows the HA distribution for all of our high-confidence matches ("true" PN with reliability > 0.8 ), parameterised by the angular size of the PN. Many compact PNe have larger HA values indicating likely nebular detections (compared to the values for extended PNe, whose detections we expect to be stellar). The lower right panel shows the distribution of all candidate matches for a few select objects. There is a clear separation between the stellar CSPN, nearby nebula detections (sources within the radius of the PN having high HA), and (usually more distant) field stars.

\footnotetext{
1 Also featured as Gaia's image of the week: https://www. cosmos. esa.int/web/gaia/iow_20141205.

2 HA values for the close visual binaries in the previous section are smaller than those of typical nebula detections; the largest is 0.26 for NGC 6818, the closest pair in the sample.
}

Nebula detections of extended PN appear rare, and to keep our approach simple, we do not use the HA in our matching. We do however include it in our catalogue as an approximate way to check how stellar-like a source is. Large values should be treated with caution, particularly in conjunction with a high photometric excess factor, but do not necessarily indicate a nebula detection.

We note that so far these detections are not useful for studies of nebular evolution. NGC 6543 for example has a measured expansion rate of $3.13 \pm 0.16 \mathrm{mas} \mathrm{yr}^{-1}$ (similar to other PNe; Schönberner et al. 2018), large enough for Gaia to detect as proper motion. However the nebula detections have only two-parameter solutions (positions only) with uncertainties much larger than any change due to expansion over Gaia's mission duration.

\section{Distances}

Distances to Galactic PNe have long been a key challenge in the study of PNe, with most distances reliant on statistical relations such as the $\mathrm{H} \alpha$ surface brightness to physical radius relation of Frew et al. (2016, hereinafter FPB16). The precision of the parallaxes in Gaia EDR3 should translate into improved precision in distance determinations, however leveraging these parallaxes is not entirely straightforward.

\subsection{Parallaxes compared to statistical distances}

Following Smith (2015), parallaxes can be used to evaluate the accuracy of a statistical distance scale using distance ratios, that is, the per-object product of statistical distance and parallax. An accurate statistical relation should yield a distance ratio distribution centred on unity. We use this method to compare Gaia parallaxes to the statistical distance scale of FPB16. Parallaxes are corrected for the zero point bias following Lindegren et al. (2021a). We use the sub-trend relations where applicable, and the same quality cuts as in CW20, with the aim of selecting high quality measurements without biasing the sample ("true" PNe, reliability $>0.98$, visibility_periods_used $>8$, RUWE $<$ 1.4 , parallax error $<0.2$ mas).

We find a median ratio of $1.03 \pm 0.02$ and a mean ratio of $1.11 \pm 0.04$ (uncertainties calculated via bootstrap). The overall distance scale is matched well (with some suggestion of the distances being slightly high), now with nearly twice as many objects being considered (294 compared to 160 in CW20). There is no statistically significant trend versus the PN surface brightness, implying the slope of the relation is reasonably accurate.

The effect of the parallax zero point correction (typically on the order of the median quasar parallax, -0.017 mas) is to make most parallaxes larger (implying smaller distances). Without the correction the distance ratios would have a smaller median value of 0.97 , and exhibit a small but significant trend with surface brightness, even after accounting for outliers. This demonstrates the need for caution interpreting parallaxes, particularly as a population in which systematics can have noticeable effects.

\subsection{Statistical distances as a prior}

Estimating distances from parallaxes is well known to require a proper prior (Luri et al. 2018). Bailer-Jones et al. (2021), hereinafter BJ21) adopted two different priors for their catalogue covering most of Gaia EDR3: one based on a galaxy model and another that additionally incorporates Gaia's photometry. Naturally, the effect of the adopted prior is greatest for stars with relatively large parallax uncertainties, which tends to result in 

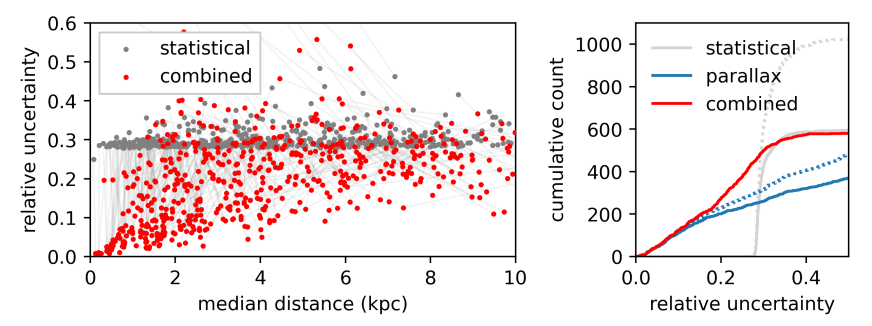

Fig. 4: Improvement over statistical distances of combined distances for "true" PNe with high-reliability matches. The left panel shows the distribution of statistical and combined distances and relative uncertainties, with lines connecting pairs of points corresponding to the same object. The right panel shows the cumulative distribution of relative uncertainties for parallaxes, statistical distances, and combined distances. The dotted lines for parallaxes and statistical distances are counts including objects with only parallaxes (not in FPB16) and all "true" PNe from FPB16 (not necessarily having parallaxes or Gaia matches) respectively.

distant, faint stars having their distances underestimated. The photometry prior aims to mitigate such effects. The rapid evolution of CSPNe makes such photometric distances less useful. However the relation between $\mathrm{H} \alpha$ surface brightness and physical size of the PN used in FPB16 can perform a similar function.

For each PN, we use the statistical distance of FPB16 and its uncertainty as a prior $P(d)$, having shown in the previous section that the FPB16 scale remains broadly consistent with the Gaia parallaxes. The Gaia parallax $\omega$ and its uncertainty $\sigma_{\omega}$ provide the likelihood $P\left(\omega \mid d, \sigma_{\omega}\right)$. We compute the posterior distance distribution on a fixed grid of $1 \mathrm{pc}$ steps between 0 and $60 \mathrm{kpc}$ (twice the largest prior distance), by simply applying Bayes' theorem, that is, taking at each distance $d$ the posterior probability to be the product $P(d) P\left(\omega \mid d, \sigma_{\omega}\right)$, normalised by the sum of these values over the domain of the prior.

Following the catalogue format adopted by BJ21, we summarise the posterior by its median and 16 th and 84 th percentiles. We publish these values in our catalogue for all 733 sources with both parallaxes and statistical distances, regardless of reliability and excess astrometric noise. Both of those should be considered when adopting the distances. There are an additional 131 sources in our catalogue that lack statistical distances but have relative parallax errors better than $20 \%$; we do not publish distances for these but expect the distances from BJ21 to not be overly influenced by their choice of prior.

Treating the width of the distributions as the difference between the 16th and 84th percentiles, the median precision improvement (relative to the median distance) over the statistical distance prior is 1.4 , with one third of the sample having its relative uncertainties improved by a factor of two or more (Fig. 4 . left). A small fraction of sources have less concentrated posteriors, typically in the case where the prior and posterior modes have a large separation. The parallaxes dominate the uncertainties below relative errors of about 0.15 (Fig. 4 , right).

The advantage of our adopted distances is that they leverage the Gaia parallaxes while smoothly transitioning to statistical distances in cases where parallaxes are unavailable or extremely uncertain. For simplicity we have only considered the mean relation of FPB16; a similar approach could be taken incorporating the sub-trends in FPB16 or even the Galactic model of BJ21. The most appropriate prior will of course depend on the application.

\section{Conclusions}

In this work we have presented a new version of our catalogue of candidate CSPNe and compact nebula detections, updated for Gaia EDR3 and including newly identified PNe from HASH. While we have made small improvements to the matching algorithm, the minimal changes required demonstrate the robustness of our original method. We have also discussed the effects of close companions and nebula detections, and introduced a novel approach to combining PN statistical distances and Gaia parallaxes.

The next Gaia data release, Gaia DR3, is expected in 2022. It is based on the exact same astrometric and photometric data as Gaia EDR3. Hence the astrometry from this Gaia EDR3 release will remain the best available for studying PNe based on their distances and kinematics until the full nominal mission Gaia data release in several years to come 3

Acknowledgements. We would like to thank the referee, W. A. Weidmann, for his comments, which have helped improve the contents and clarity of this paper. This research has made use of data from the European Space Agency (ESA) mission Gaia (https://www.cosmos.esa.int/gaia), processed by the Gaia Data Processing and Analysis Consortium (DPAC; https://www. cosmos.esa.int/web/gaia/dpac/consortium). Funding for the DPAC has been provided by national institutions, in particular the institutions participating in the Gaia Multilateral Agreement. This research has also made use of the HASH PN database (http://hashpn.space), and of Astropy (http:// WWW . astropy.org), a community-developed core Python package for Astronomy (Astropy Collaboration et al. 2013. Price-Whelan et al. 2018). Parts of this research were based on data products from observations made with ESO Telescopes at the La Silla Paranal Observatory under programme ID 177.D-3023, as part of the VST Photometric $\mathrm{H} \alpha$ Survey of the southern Galactic plane and bulge (VPHAS+, www.vphas.eu). This research was supported through the Cancer Research UK grant A24042.

\section{References}

Astropy Collaboration, Robitaille, T. P., Tollerud, E. J., et al. 2013, A\&A, 558, A33

Bailer-Jones, C. A. L., Rybizki, J., Fouesneau, M., Demleitner, M., \& Andrae, R. 2021, AJ, 161, 147

Benetti, S., Cappellaro, E., Ragazzoni, R., Sabbadin, F., \& Turatto, M. 2003, A\&A, 400, 161

Chornay, N. \& Walton, N. A. 2020, A\&A, 638, A103

Ciardullo, R., Bond, H. E., Sipior, M. S., et al. 1999, AJ, 118, 488

Drew, J. E., Gonzalez-Solares, E., Greimel, R., et al. 2014, MNRAS, 440, 2036 Evans, D. W., Riello, M., De Angeli, F., et al. 2018, A\&A, 616, A4

Fabricius, C., Bastian, U., Portell, J., et al. 2016, A\&A, 595, A3

Fabricius, C., Luri, X., Arenou, F., et al. 2021, A\&A, 649, A5

Frew, D. J., Parker, Q. A., \& Bojičić, I. S. 2016, MNRAS, 455, 1459

Gaia Collaboration, Brown, A. G. A., Vallenari, A., et al. 2018, A\&A, 616, A1

Gaia Collaboration, Brown, A. G. A., Vallenari, A., et al. 2021, A\&A, 649, A1

Gaia Collaboration, Prusti, T., de Bruijne, J. H. J., et al. 2016, A\&A, 595, A1

González-Santamaría, I., Manteiga, M., Manchado, A., et al. 2020, A\&A, 644, A173

Liebert, J., Bond, H. E., Dufour, P., et al. 2013, ApJ, 769, 32

Lindegren, L. 2018, GAIA-C3-TN-LU-LL-124

Lindegren, L., Bastian, U., Biermann, M., et al. 2021a, A\&A, 649, A4

Lindegren, L., Klioner, S. A., Hernández, J., et al. 2021b, A\&A, 649, A2

Luri, X., Brown, A. G. A., Sarro, L. M., et al. 2018, A\&A, 616, A9

Parker, Q. A., Acker, A., Frew, D. J., et al. 2006, Monthly Notices of the Royal Astronomical Society, 373, 79

Parker, Q. A., Bojičić, I. S., \& Frew, D. J. 2016, in Journal of Physics Conference Series, Vol. 728, Journal of Physics Conference Series, 032008

Price-Whelan, A. M., Sipőcz, B. M., Günther, H. M., et al. 2018, AJ, 156, 123

Riello, M., De Angeli, F., Evans, D. W., et al. 2021, A\&A, 649, A3

Schönberner, D., Balick, B., \& Jacob, R. 2018, A\&A, 609, A126

Smith, H. 2015, MNRAS, 449, 2980

Sutherland, W. \& Saunders, W. 1992, MNRAS, 259, 413

Torra, F., Castañeda, J., Fabricius, C., et al. 2021, A\&A, 649, A10

Weidmann, W. A. \& Gamen, R. 2011, A\&A, 526, A6

\footnotetext{
3 See https://www. cosmos.esa.int/web/gaia/release for the official release schedule.
} 
Appendix A: Best matches table 
Table A.1: Best matches ${ }^{4}$

\begin{tabular}{|c|c|c|c|c|c|c|c|c|c|}
\hline$\overline{\mathrm{PNG}}$ & PN name & Gaia source ID & reliability & $\begin{array}{c}\text { RA } \\
(\mathrm{deg})\end{array}$ & $\begin{array}{l}\text { Dec } \\
(\mathrm{deg})\end{array}$ & $\begin{array}{c}\mathrm{G} \\
(\mathrm{mag})\end{array}$ & $\begin{array}{c}w^{5} \\
(\mathrm{mas})\end{array}$ & $\begin{array}{l}\sigma_{a} \sqrt{6} \\
(\mathrm{mas})\end{array}$ & 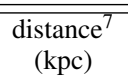 \\
\hline $000.0+02.0$ & K 6-4 & 4060525376634471808 & 0.65 & 264.42892 & -27.81947 & 19.5 & & & $\mathrm{~T}^{2}$ \\
\hline $000.0-01.0$ & JaSt 69 & 4057224264723323264 & 0.26 & 267.54157 & -29.31827 & 19.7 & -0.817 & 0.396 & \\
\hline $000.0-06.8$ & H 1-62 & 4045771305065496832 & 0.98 & 273.32482 & -32.32860 & 14.4 & 0.047 & 0.034 & $8.95_{-147}^{+1.73}$ \\
\hline $000.1+04.3$ & H $1-16$ & 4109679250049965312 & 0.98 & 262.34731 & -26.43461 & 18.7 & & & \\
\hline $000.1+17.2$ & PC 12 & 4130784921205604736 & 1.00 & 250.97408 & -18.95333 & 15.2 & 0.069 & 0.066 & $9.86_{-3,58}^{+3.01}$ \\
\hline $000.1-05.6$ & H 2-40 & 4049045783774253696 & 0.57 & 272.12813 & -31.60981 & 18.5 & 0.340 & 0.268 & $5.94_{-1.94}^{+2.01}$ \\
\hline $000.1-08.0$ & SB 1 & 4045583322840954496 & 1.00 & 274.70193 & -32.79860 & 20.0 & -0.228 & 0.616 & $\ldots$ \\
\hline $000.2+01.4$ & PBOZ 26 & 4060650446035698944 & 0.75 & 265.16073 & -28.01722 & 21.1 & ... & $\ldots$ & $\ldots$ \\
\hline $000.2+01.7$ & JaSt 19 & 4060845334555887616 & 0.88 & 264.91380 & -27.78946 & 20.8 & & & \\
\hline $000.2-01.9$ & M 2-19 & 4056495151130724224 & 0.42 & 268.44019 & -29.72971 & 16.9 & -0.164 & 0.112 & $6.41^{+1.27}$ \\
\hline $000.3+01.5$ & JaSt 23 & 4060841383089804544 & 0.97 & 265.09603 & -27.81998 & 21.1 & & & \\
\hline $000.3+02.5$ & MPA1736-2715 & 4061311767987964672 & 0.65 & 264.12350 & -27.25573 & 17.4 & -0.114 & 0.144 & $\ldots$ \\
\hline $000.3+02.7$ & $\begin{array}{l}\text { CTIOJ173557.97- } \\
271214.2\end{array}$ & 4061324584115092736 & 0.61 & 263.99148 & -27.20386 & 19.8 & & & . \\
\hline $000.3+03.2$ & PHR J1733-2655 & 4061390245613519232 & 0.66 & 263.46913 & -26.92419 & 20.4 & -0.645 & 1.101 & $\ldots$ \\
\hline $000.3+03.4$ & PHR J1733-2647 & 4061581663670658048 & 0.66 & 263.36382 & -26.80004 & 20.4 & & & \\
\hline $000.3+04.2$ & PPA J1730-2621 & 4061648012333306752 & 0.31 & 262.55146 & -26.35036 & 18.5 & 0.208 & 0.240 & \\
\hline $000.3+12.2$ & IC 4634 & 4126115570219432448 & 0.99 & 255.38992 & -21.82586 & 13.9 & 0.370 & 0.043 & $2.50^{+0.29}$ \\
\hline $000.3-01.7$ & $\begin{array}{l}\text { CTIOJ175310.09- } \\
293108.6\end{array}$ & 4056550298602369408 & 0.85 & 268.29201 & -29.51913 & 20.4 & & 0.078 & $\ldots$ \\
\hline $000.3-03.0$ & $\mathrm{~Pa} 201$ & 4056234017106233600 & 0.66 & 269.66975 & -30.16738 & 15.3 & 0.129 & 0.039 & $\ldots$ \\
\hline $000.3-03.4$ & MPA J1800-3023 & 4044204947649634432 & 0.50 & 270.04623 & -30.39709 & 18.1 & 0.326 & 0.219 & $\ldots$ \\
\hline $000.3-04.2$ & MPA J1803-3043 & 4049954873646647040 & 0.55 & 270.84046 & -30.72654 & 15.4 & 0.153 & 0.045 & $\ldots$ \\
\hline $000.3-04.2 \mathrm{a}$ & MPA J1803-3046 & 4049951270196738816 & 0.57 & 270.85747 & -30.77349 & 20.3 & & . & $\ldots$ \\
\hline $000.3-04.6$ & M 2-28 & 4049886772877581696 & 0.57 & 271.26114 & -30.97130 & 19.6 & & & $\ldots$ \\
\hline $000.4+02.2$ & PM 1-165 & 4060929374082309888 & 0.48 & 264.53102 & -27.33750 & 17.5 & 0.051 & 0.305 & \\
\hline $000.4+03.3$ & $\begin{array}{ll}\text { CTIO } & \text { J173348.40- } \\
264623.4 & \end{array}$ & 4061581526220991232 & 0.91 & 263.45167 & -26.77320 & 19.8 & -0.392 & 0.818 & 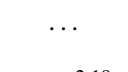 \\
\hline $000.4+04.4$ & K 5-1 & 4109691718340733568 & 0.90 & 262.46816 & -26.18713 & 19.2 & -0.164 & 0.395 & $7.03_{-2.10}^{+2.19}$ \\
\hline $000.4-01.9$ & M 2-20 & 4056503259966429696 & 0.99 & 268.60570 & -29.60244 & 17.2 & & & \\
\hline $000.4-02.9$ & M 3-19 & 4056250956464755712 & 0.48 & 269.58079 & -30.01094 & 18.3 & 0.367 & 0.189 & $4.57^{+1.51}$ \\
\hline $000.5-03.1$ & KFL 1 & 4056241095169816448 & 0.87 & 269.81499 & -30.04636 & 19.1 & -3.626 & 0.733 & $7.14_{-187}^{+1.97}$ \\
\hline $000.5-03.1 \mathrm{a}$ & MPA J1759-3007 & 4056239308511338240 & 0.64 & 269.85494 & -30.12166 & 19.1 & & & -1. \\
\hline $000.6-01.0$ & JaSt 77 & 4057342599736948992 & 0.91 & 267.79797 & -28.94061 & 16.1 & 0.336 & 0.088 & \\
\hline $000.6-01.3$ & B1 3-15 & 4056579538679085952 & 0.96 & 268.15023 & -29.11101 & 19.3 & -0.268 & 0.474 & $9.11_{-3.24}^{+3.45}$ \\
\hline $000.6-02.3$ & H 2-32 & 4056324962986093184 & 0.37 & 269.10134 & -29.63524 & 15.1 & 0.111 & 0.036 & $\ldots$ \\
\hline $000.6-03.2$ & MPA J1759-3004 & 4044231610846145408 & 0.23 & 269.98615 & -30.07469 & 19.5 & & & . \\
\hline $000.6-04.5$ & PN PM 1-206 & 4049944565824708608 & 0.93 & 271.30196 & -30.70368 & 18.3 & -0.699 & 0.345 & $\ldots$ \\
\hline $000.7+03.2$ & M 4-5 & 4061777617281328896 & 0.82 & 263.72799 & -26.59926 & 20.9 & & & $\ldots$ \\
\hline $000.7+04.7$ & H 2-11 & 4109783360108033024 & 0.85 & 262.35817 & -25.81867 & 19.1 & & & $\ldots$ \\
\hline $000.7+08.0$ & MPA J1717-2356 & 4114088875802922880 & 0.94 & 259.28771 & -23.94158 & 18.9 & 0.062 & 0.254 & $\ldots$ \\
\hline $000.7-01.5$ & JaSt 2-11 & 4056576446240230656 & 0.57 & 268.36253 & -29.13790 & 19.4 & & & \\
\hline $000.7-03.7$ & M 3-22 & 4050168629923554944 & 0.77 & 270.58023 & -30.24053 & 18.4 & 1.391 & 0.299 & $0.94^{+1.53}$ \\
\hline $000.7-06.1$ & SB 3 & 4048968994096810880 & 1.00 & 273.06065 & -31.33348 & 19.0 & -0.357 & 0.259 & $4.19_{-0.89}^{+0.96}$ \\
\hline $000.7-07.4$ & M 2-35 & 404 & 0.96 & 274.40498 & -31.94639 & 19.9 & & & \\
\hline $000.8+05.2$ & PBOZ 5 & 4109999173694500992 & 0.62 & 261.91057 & -25.37405 & 19.7 & 4.818 & 1.503 & \\
\hline $000.8-01.5$ & Sa 3-90 & 4056603178196321792 & 0.38 & 268.45721 & -28.98657 & 16.5 & 0.045 & 0.072 & $10.99_{-264}^{+2.86}$ \\
\hline $000.8-07.6$ & H 2-46 & 4045845625148544640 & 0.96 & 274.65605 & -31.91259 & 18.9 & & & $\ldots$ \\
\hline $000.9-00.7$ & JaSt 76 & 497334644864 & 0.32 & 267.73495 & -28.52350 & 17.7 & 0.119 & 0.119 & $\ldots$ \\
\hline $000.9-01.0$ & MPA J1751-2838 & 4057379398969699328 & 0.53 & 267.93021 & -28.64963 & 20.7 & & & $\ldots$ \\
\hline $000.9-01.4$ & JaSt 2-13 & 4056607713616891648 & 0.27 & 268.39898 & -28.87314 & 18.9 & -0.727 & 0.401 & \\
\hline $000.9-01.9 \mathrm{a}$ & MPA1755-2906 & 4056544903986265856 & 0.36 & 268.94630 & -29.11119 & 19.5 & & & \\
\hline $000.9-02.0$ & B1 3-13 & 4056540677880158208 & 0.65 & 269.01160 & -29.18796 & 18.0 & 0.167 & 0.183 & $7.40_{-2.68}^{+3.08}$ \\
\hline $000.9-04.2$ & PHR J1804-3016 & 4049992845542438912 & 0.85 & 271.20063 & -30.28038 & 19.4 & 3.308 & 0.693 & \\
\hline $000.9-04.8$ & M 3-23 & 4049925328633027712 & 0.98 & 271.77560 & -30.57141 & 19.2 & 0.253 & 0.383 & $4.37^{+1.25}$ \\
\hline $000.9-05.4$ & PN PM 1-214 & 869657862656 & 0.95 & 272.40835 & -30.81458 & 17.5 & 0.428 & 0.114 & 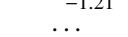 \\
\hline $001.0+01.3$ & JaSt 41 & 4060698034329272832 & 0.77 & 265.70770 & -27.35538 & 21.0 & & & $\ldots$ \\
\hline $001.0+01.4$ & JaSt 2-4 & 4060890513224820352 & 0.44 & 265.61691 & -27.22550 & 16.0 & 0.708 & 0.064 & \\
\hline $001.0+01.9$ & K $1-4$ & 4060952605654047232 & 0.93 & 265.11420 & -27.01727 & 20.2 & $\ldots$ & $\ldots$ & \\
\hline $001.0+02.2$ & PPA J1739-2652 & 4061719789876312704 & 0.72 & 264.79697 & -26.87232 & 20.0 & .. & $\ldots$ & $\ldots$ \\
\hline $001.0-01.5$ & MPA1754-2847 & 4056614001551704192 & 0.37 & 268.53925 & -28.78968 & 20.2 & & & \\
\hline $001.1+02.2$ & MPA J1739-2648 & 4060976176417193856 & 0.34 & 264.95709 & -26.81264 & 19.6 & 0.392 & 0.524 & \\
\hline $001.1-01.2$ & JaSt $2-10$ & 4057371186977715968 & 0.85 & 268.32247 & -28.60203 & 20.2 & & & \\
\hline $001.1-01.6$ & Sa 3-92 & 4056611390167199232 & 0.57 & 268.71713 & -28.81534 & 19.3 & -2.150 & 0.899 & $10.09_{-2 .}^{+2.8}$ \\
\hline $001.1-02.6$ & MPA1758-2915 & 4062332454138572416 & 0.25 & 269.71511 & -29.26625 & 18.6 & 0.500 & 0.311 & \\
\hline
\end{tabular}

\footnotetext{
${ }^{4}$ Only the most important columns are shown due to space considerations. The complete set of columns will be published on CDS, and missing columns may also be obtained by joining the table against Gaia EDR3 and HASH.

5 Parallax, not corrected for zero point.

${ }^{6}$ Parallax errror.

7 Median value of the posterior; upper and lower bounds are 84th and 16th percentiles. See Sect. 4.2
} 
Table A.1: continued.

\begin{tabular}{|c|c|c|c|c|c|c|c|c|c|}
\hline PN G & PN name & Gaia source ID & reliability & $\begin{array}{c}\text { RA } \\
\text { (deg) }\end{array}$ & $\begin{array}{l}\text { Dec } \\
\text { (deg) }\end{array}$ & $\begin{array}{c}\mathrm{G} \\
(\mathrm{mag})\end{array}$ & $\begin{array}{c}\omega \\
(\mathrm{mas})\end{array}$ & $\begin{array}{c}\sigma_{\omega} \\
(\mathrm{mas})\end{array}$ & $\begin{array}{c}\text { distance } \\
(\mathrm{kpc})\end{array}$ \\
\hline $001.1-11.5$ & PPA1835-3324 & 6735044388453345408 & 0.73 & 278.93112 & -33.41245 & 17.8 & 0.043 & 0.124 & $\ldots$ \\
\hline $001.2+00.7$ & JaSt 56 & 4060726690316450432 & 0.42 & 266.44534 & -27.51165 & 21.0 & & & \\
\hline $001.2+00.9$ & St 66 & 4057436539233503104 & 0.48 & 268.03558 & -28.39386 & 17.9 & 0.255 & 0.135 & $\ldots$ \\
\hline $001.2+01.0$ & K 5-33 & 4060777439619558528 & 0.51 & 266.12477 & -27.34456 & 18.6 & 0.279 & 0.336 & \\
\hline $001.2+02.1$ & Hen 2-262 & 4060978306741619968 & 0.90 & 265.05347 & -26.73938 & 18.7 & -0.116 & 0.404 & $5.91_{-1.71}^{+1.79}$ \\
\hline $001.2+02.8$ & PPA J1737-2621 & 4061796583872776576 & 0.48 & 264.37631 & -26.36209 & 17.0 & 0.140 & 0.085 & \\
\hline $001.2-01.2 \mathrm{a}$ & JaSt 95 & 4057385514989465216 & 0.53 & 268.39701 & -28.48084 & 18.2 & 0.638 & 0.247 & $6.13_{-301}^{+2.91}$ \\
\hline $001.2-03.0$ & H $1-47$ & 4062301564840251520 & 0.49 & 270.15676 & -29.36410 & 15.7 & 0.091 & 0.052 & $8.05_{-1.36}^{+2.01}$ \\
\hline $001.2-03.8$ & PHR J1803-2947 & 4050258102727839488 & 0.51 & 270.87408 & -29.78246 & 19.6 & & & \\
\hline $001.2-05.6$ & PHR J1811-3042 & 4049240298544263936 & 1.00 & 272.76129 & -30.70329 & 18.6 & 0.667 & 0.422 & $6.35_{-1.94}^{+1.92}$ \\
\hline $001.3+04.0$ & IRAS17301-2538 & 4062070460993016064 & 0.93 & 263.30497 & -25.67286 & 19.5 & & & \\
\hline $001.3-01.2$ & $\mathrm{Bl} \mathrm{M}$ & 4063378574972871680 & 0.69 & 268.44655 & -28.45503 & 18.0 & 0.751 & 0.297 & $4.52_{-270}^{+3.88}$ \\
\hline $001.3-05.6$ & SB 5 & 4049264242907686016 & 1.00 & 272.81422 & -30.63056 & 15.5 & -0.014 & 0.046 & -2. \\
\hline $001.4+00.5$ & [GKF2010] MN66 & 4060739369060593152 & 0.65 & 266.77174 & -27.42611 & 17.9 & 0.543 & 0.123 & $\ldots$ \\
\hline $001.4+05.3$ & H $1-15$ & 4110404068789630592 & 0.68 & 262.15676 & -24.85202 & 15.8 & 0.150 & 0.045 & 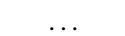 \\
\hline $001.4+06.3$ & Bica 1 & 4110871567379348480 & 0.72 & 261.22407 & -24.32237 & 20.7 & & & $\ldots$ \\
\hline $001.4-00.7$ & JaSt 82 & 4057456261747292672 & 0.23 & 268.02203 & -28.09646 & 20.6 & & . & $\ldots$ \\
\hline $001.4-03.4$ & ShWi 2-1 & 4050281914036492544 & 0.53 & 270.61021 & -29.41821 & 19.8 & & & \\
\hline $001.5+01.5$ & JaSt 46 & 4061009264777169920 & 0.78 & 265.87646 & -26.79241 & 20.0 & & & $\ldots$ \\
\hline $001.5+03.6$ & K 5-5 & 4062080223477010048 & 0.99 & 263.84212 & -25.71305 & 19.7 & -1.279 & 0.807 & $\ldots$ \\
\hline $001.5-01.0$ & JaSt 2-12 & 4063398469194112256 & 0.29 & 268.36500 & -28.19704 & 19.7 & & & - \\
\hline $001.5-01.8$ & JaSt 2-19 & 4062615891871616000 & 0.38 & 269.14080 & -28.50814 & 19.3 & & & \\
\hline $001.5-02.8$ & PPA J1800-2904 & 4062356711999251328 & 0.78 & 270.09344 & -29.07756 & 18.1 & 0.382 & 0.199 & \\
\hline $001.5-06.7$ & SwSt 1 & 4049331244394134912 & 0.97 & 274.05115 & -30.86887 & 11.8 & 0.343 & 0.110 & $3.11_{-066}^{+0.81}$ \\
\hline $001.6+01.5$ & K 6-10 & 4061013383646090624 & 0.91 & 265.82060 & -26.73819 & 19.3 & 1.054 & 0.374 & $6.13_{-3.17}^{+2.79}$ \\
\hline $001.6-01.0$ & JaSt 90 & 4063447294418877056 & 0.55 & 268.32395 & -28.07589 & 19.5 & -0.256 & 1.184 & \\
\hline $001.6-03.7$ & MPA J1804-2926 & 4050361456844630656 & 0.22 & 271.04368 & -29.44047 & 19.2 & & $\ldots$ & $\ldots$ \\
\hline $001.7+00.7$ & PBOZ 14 & 4060813345574048000 & 0.21 & 266.68372 & -27.05634 & 21.0 & & & \\
\hline $001.7+01.3$ & JaSt 52 & 4061018988593063040 & 0.82 & 266.15509 & -26.79042 & 18.1 & 0.233 & 0.225 & $6.79_{-2.41}^{+2.61}$ \\
\hline $001.7+05.7$ & H $1-14$ & 4110479900747426944 & 0.89 & 262.00726 & -24.42318 & 19.6 & -0.293 & 0.530 & $\ldots$ \\
\hline $001.7-00.3$ & $\mathrm{MC1}$ & 4063675339961746304 & 0.22 & 267.76894 & -27.61627 & 20.8 & & & 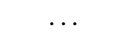 \\
\hline $001.7-01.6$ & H 2-31 & 4062657913789180672 & 0.91 & 269.00998 & -28.23675 & 17.7 & 0.067 & 0.183 & \\
\hline $001.7-04.4$ & H 1-55 & 4050131349653595392 & 0.94 & 271.81065 & -29.69017 & 16.6 & 0.036 & 0.860 & $9.68_{-3.29}^{+3.32}$ \\
\hline $001.7-04.6$ & H $1-56$ & 4050126711087206016 & 0.98 & 271.97450 & -29.74291 & 16.0 & 0.092 & 0.062 & $8.98_{-2.09}^{+2.39}$ \\
\hline $001.8-00.4$ & MB 4515 & 4063669086488636928 & 0.99 & 267.97728 & -27.60295 & 20.0 & -0.679 & 0.507 & $\ldots$ \\
\hline $001.8-00.5$ & JaSt 81 & 4063668399280242560 & 0.26 & 268.01784 & -27.61056 & 19.3 & -0.404 & 0.401 & \\
\hline $001.8-01.5$ & JaSt 2-18 & 4063411732140228096 & 0.22 & 268.97680 & -28.09676 & 20.6 & . & - & .. \\
\hline $001.8-01.5 \mathrm{a}$ & PNG001.82-01.5 & 4063410082883861376 & 0.30 & 268.93672 & -28.13914 & 21.3 & & & \\
\hline $001.8-03.7$ & PHR J1804-2913 & 4050381621840757760 & 0.95 & 271.11879 & -29.23260 & 16.2 & -0.204 & 0.099 & $18.53_{-430}^{+4.55}$ \\
\hline $001.8-05.0$ & PHR J1809-2952 & 4050066203563096704 & 0.51 & 272.44551 & -29.88147 & 18.2 & & & $\ldots$ \\
\hline $001.9+02.3$ & K 5-10 & 4061866089277230848 & 0.72 & 265.35216 & -26.06482 & 20.4 & -0.553 & 1.073 & $\ldots$ \\
\hline $001.9+08.2$ & PM 1-139 & 4114675327843171456 & 0.83 & 259.85934 & -22.80317 & 19.7 & -0.088 & 0.482 & 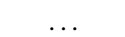 \\
\hline $001.9-02.5$ & PPA J1759-2834 & 4062669458655947648 & 0.99 & 269.96952 & -28.57989 & 19.4 & 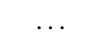 & 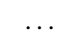 & $\ldots$ \\
\hline $002.0+02.1$ & [GKF2010] MN60 & 4061870010605672832 & 0.54 & 265.53128 & -26.03686 & 20.3 & & & $\ldots$ \\
\hline $002.0+06.6$ & PHR J1725-2338 & 4111350267272130560 & 0.53 & 261.42410 & -23.64201 & 19.0 & 0.961 & 0.541 &  \\
\hline $002.0-01.3$ & JaSt 98 & 4063434753111065472 & 0.98 & 268.94279 & -27.89395 & 20.6 & & & \\
\hline $002.0-03.4$ & PPA J1803-2855 & 4062403170636645376 & 0.96 & 270.90805 & -28.92820 & 18.5 & 0.073 & 0.269 & \\
\hline $002.0-06.2$ & M 2-33 & 4049596604655713664 & 0.97 & 273.77735 & -30.25927 & 14.7 & 0.241 & 0.042 & $4.05_{-063}^{+0.91}$ \\
\hline $002.0-13.4$ & IC 4776 & 6735622632789070976 & 0.98 & 281.46131 & -33.34285 & 13.0 & & & \\
\hline $002.1+01.7$ & $\mathrm{JaFu} 1$ & 4061102036129908480 & 0.20 & 265.98826 & -26.19801 & 20.2 & -2.261 & 2.033 & $7.21_{-2.30}^{+2.33}$ \\
\hline $002.1+03.3$ & PBOZ 24 & 4062163301005727616 & 0.22 & 264.46309 & -25.34599 & 19.8 & -0.565 & 0.467 & \\
\hline $002.1-00.9$ & K 5-35 & 4063498834054665600 & 0.47 & 268.57850 & -27.60994 & 15.9 & 0.167 & 0.215 & \\
\hline $002.1-01.1$ & MPA J1755-2741 & 4063488045115810048 & 0.86 & 268.79332 & -27.69439 & 19.5 & 1.573 & 0.565 & \\
\hline $002.1-02.2$ & M 3-20 & 4062693716586525952 & 0.86 & 269.83054 & -28.23008 & 17.7 & $\ldots$ & $\ldots$ & $\ldots$ \\
\hline $002.1-02.4$ & PPA J1800-2818 & 4062682755878853248 & 0.76 & 270.07846 & -28.30969 & 19.2 & & & \\
\hline $002.1-04.2$ & H $1-54$ & 4050346677743390080 & 0.57 & 271.78030 & -29.21834 & 15.0 & 0.349 & 0.142 & $6.61_{-2.54}^{+2.73}$ \\
\hline $002.1-08.3$ & PPA J1824-3107 & 4046186679873669504 & 1.00 & 276.09064 & -31.11937 & 17.9 & 0.275 & 0.154 & $\ldots$ \\
\hline $002.2+01.7$ & PPA J1744-2605 & 4067108221452898816 & 0.84 & 266.00695 & -26.09598 & 19.8 & & & $\ldots$ \\
\hline $002.2-01.7$ & PN PM 1-195 & 4062764085412604672 & 0.61 & 269.49290 & -27.88901 & 19.7 & 2.559 & 1.001 & 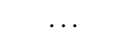 \\
\hline $002.2-02.5$ & KFL 2 & 4062681793803938688 & 0.89 & 270.24907 & -28.27195 & 19.5 & $\ldots$ & $\ldots$ & $\ldots$ \\
\hline $002.2-02.7$ & M 2-23 & 4062488485983362944 & 0.93 & 270.42767 & -28.42892 & 14.2 & & & . \\
\hline $002.2-04.3$ & K 6-40 & 4050710792186748288 & 0.21 & 272.02732 & -29.21972 & 19.9 & -0.271 & 1.237 & \\
\hline $002.2-06.3$ & H 1-63 & 4049624646596477312 & 0.99 & 274.08059 & -30.12671 & 13.4 & 0.430 & 0.598 & $8.24_{-2.55}^{+2.55}$ \\
\hline $002.2-09.4$ & Cn 1-5 & 4046842607233523840 & 1.00 & 277.29853 & -31.49978 & 15.2 & 0.276 & 0.086 & $4.21_{-0.98}^{+1.23}$ \\
\hline $002.3+02.4$ & PPA J1741-2538 & 4061894715268479872 & 0.67 & 265.45168 & -25.63846 & 19.7 & 2.309 & 1.169 & $\ldots$ \\
\hline $002.3+03.6$ & PPA J1737-2501 & 4062219616618683776 & 0.92 & 264.34986 & -25.02557 & 19.9 & 2.550 & 0.610 & \\
\hline $002.3+06.2$ & PPA J1727-2338 & 4111387680667596288 & 0.96 & 261.92383 & -23.64812 & 19.7 & -0.546 & 0.611 & \\
\hline $002.3+06.5$ & PPA J1726-2325 & 4111359780579498368 & 0.29 & 261.64101 & -23.42078 & 20.1 & $\ldots$ & $\ldots$ & $\ldots$ \\
\hline $002.3+06.5 \mathrm{a}$ & PHR J1726-2326 & 4111359406964059392 & 0.62 & 261.65176 & -23.44401 & 20.6 & & & \\
\hline $002.3-03.4$ & H 2-37 & 4062437324328722304 & 0.89 & 271.12005 & -28.62731 & 18.9 & & & \\
\hline $002.3-05.9$ & PPA J1814-2951 & 4049688658764663552 & 1.00 & 273.61125 & -29.86300 & 18.0 & 0.373 & 0.169 & \\
\hline $002.3-07.8$ & M 2-41 & 4046378407157239040 & 0.61 & 275.64371 & -30.72481 & 18.8 & -0.423 & 0.210 & $7.35_{-158}^{+1.67}$ \\
\hline
\end{tabular}


Table A.1: continued.

\begin{tabular}{|c|c|c|c|c|c|c|c|c|c|}
\hline PN G & PN name & Gaia source ID & reliability & $\begin{array}{c}\text { RA } \\
\text { (deg) }\end{array}$ & $\begin{array}{c}\text { Dec } \\
(\mathrm{deg})\end{array}$ & $\begin{array}{c}\mathrm{G} \\
(\mathrm{mag})\end{array}$ & $\begin{array}{c}\omega \\
(\mathrm{mas})\end{array}$ & $\begin{array}{c}\sigma_{\omega} \\
(\mathrm{mas})\end{array}$ & $\begin{array}{c}\text { distance } \\
(\mathrm{kpc})\end{array}$ \\
\hline $002.4+05.8$ & NGC 6369 & 4111368477921050368 & 0.78 & 262.33523 & -23.75966 & 15.5 & 0.918 & 0.049 & $1.04^{+0.06}$ \\
\hline $002.4+07.0$ & PHR J1724-2302 & 4111575014353546240 & 0.50 & 261.22175 & -23.04298 & 16.2 & 0.189 & 0.056 & $\ldots$ \\
\hline $002.4-01.7$ & PBOZ 32 & 4063524496437676288 & 0.25 & 269.55023 & -27.68933 & 20.2 & & & \\
\hline $002.4-03.1$ & PPA J1803-2826 & 4062496349974862464 & 0.92 & 270.85296 & -28.44061 & 16.5 & 0.109 & 0.080 & $\ldots$ \\
\hline $002.4-03.4$ & PHR J1804-2833 & 4062438694311477632 & 0.77 & 271.23076 & -28.55249 & 13.9 & 0.211 & 0.031 & \\
\hline $002.4-03.7$ & M 1-38 & 4050798924999155456 & 0.98 & 271.52394 & -28.67502 & 14.1 & 0.120 & 0.026 & $6.52_{-089}^{+1.13}$ \\
\hline $002.4-05.0$ & K 6-41 & 4050459652633253376 & 0.54 & 272.80225 & -29.36675 & 18.5 & -2.029 & 0.509 & \\
\hline $002.5+01.9$ & PHR J1744-2545 & 4067134609739799552 & 0.24 & 266.00100 & -25.76004 & 20.0 & 1.128 & 0.808 & $\ldots$ \\
\hline $002.5+04.9$ & PHR J1732-2408 & 4110559855799571200 & 0.73 & 263.24253 & -24.14594 & 19.7 & 0.078 & 0.542 & $\ldots$ \\
\hline $002.5-01.7$ & Pe 2-11 & 4063525217993978496 & 0.38 & 269.63008 & -27.61795 & 19.6 & & &  \\
\hline $002.6+02.3$ & PHR J1742-2525 & 4067908184848743936 & 0.44 & 265.71725 & -25.42856 & 19.8 & -0.643 & 0.465 & $\ldots$ \\
\hline $002.6+04.2$ & Th 3-27 & 4110306865077121664 & 0.90 & 263.99364 & -24.42485 & 19.4 & & & \\
\hline $002.6+04.8$ & PHR J1733-2407 & 4110558859449807488 & 0.81 & 263.40743 & -24.12952 & 16.0 & 0.007 & 0.051 & \\
\hline $002.6+05.5$ & K 5-3 & 4110623760607130624 & 0.58 & 262.67188 & -23.75011 & 19.2 & 0.200 & 0.337 & $5.54_{-1}^{+1.64}$ \\
\hline $002.6+08.1$ & H 1-11 & 4114722469423030272 & 0.96 & 260.32383 & -22.30988 & 16.8 & 0.214 & 0.074 & $5.82_{-1.49}^{+1.89}$ \\
\hline $002.6-01.7$ & PHR J1758-2729 & 4063550678552452096 & 0.24 & 269.62060 & -27.49472 & 17.9 & 0.762 & 0.154 & \\
\hline $002.6-03.4$ & M 1-37 & 4062817927079802240 & 0.96 & 271.35751 & -28.36789 & 14.8 & 0.237 & 0.043 & $4.05_{-065}^{+1.00}$ \\
\hline $002.7-04.8$ & M 1-42 & 4050550916418916864 & 0.73 & 272.77079 & -28.98309 & 18.3 & -1.359 & 0.524 & $4.31_{-1.03}^{+1.10}$ \\
\hline $002.7-11.3$ & CGMW 4-2085 & 6736781926089349760 & 1.00 & 279.53227 & -31.88254 & 19.8 & -0.122 & 0.529 & \\
\hline $002.7-52.4$ & IC $5148 / 50$ & 6574225217863069056 & 1.00 & 329.89623 & -39.38566 & 16.1 & 0.857 & 0.053 & $1.18_{-0.07}^{+0.08}$ \\
\hline $002.8+01.7$ & H 2-20 & 4067157424631394944 & 0.44 & 266.41582 & -25.66681 & 17.8 & -0.148 & 0.160 & $11.66_{-3.34}^{+3.62}$ \\
\hline $002.8-10.7$ & PHR J1835-3135 & 4046697815310010752 & 0.98 & 278.89035 & -31.59580 & 19.4 & 0.726 & 0.311 & \\
\hline $002.9+06.5$ & PM 1-149 & 4111493787901120768 & 0.91 & 261.97369 & -22.95520 & 15.6 & 0.195 & 0.039 & \\
\hline $002.9-03.9$ & H 2-39 & 4050818097742912640 & 0.99 & 272.02404 & -28.43634 & 17.8 & -0.554 & 0.216 & $10.27_{-2.39}^{+2.52}$ \\
\hline $002.9-07.0$ & PPA J182 & 4049507239329506560 & 0.46 & 275.14754 & -29.81377 & 18.8 & 0.738 & 0.562 & $20.29_{-6.05}^{+6.00}$ \\
\hline $002.9-10.2$ & PHR J1833-3115 & 4046908436166480768 & 0.87 & 278.42825 & -31.26194 & 17.6 & -0.361 & 0.185 & \\
\hline $003.0+01.1$ & Kn J1748.1-2544 & 4067190478657457920 & 0.88 & 267.02592 & -25.73627 & 19.2 & & & \\
\hline $003.0-05.0$ & PHR J1812-2848 & 4050641243893555200 & 0.90 & 273.09751 & -28.80269 & 19.0 & & & \\
\hline $003.1+01.9$ & PPA J1745-2514 & 4067924437022290176 & 0.50 & 266.41638 & -25.23500 & 19.6 & 0.190 & 0.669 & \\
\hline $003.1+02.9$ & $\mathrm{Hb} 4$ & 4068200586367730944 & 1.00 & 265.47000 & -24.70233 & 17.5 & 0.165 & 0.132 & $3.39_{-0.63}^{+0.70}$ \\
\hline $003.1+03.4$ & H 2-17 & 4068268859125380224 & 0.93 & 265.03092 & -24.42853 & 16.5 & 0.083 & 0.062 & $7.47_{-1.53}^{+1.95}$ \\
\hline $003.1+03.7$ & PHR J1738-2419 & 4068263155407067904 & 0.91 & 264.71536 & -24.32890 & 16.6 & 0.109 & 0.114 & $\ldots$ \\
\hline $003.1+04.1$ & K 5-7 & 4116324247383734656 & 0.98 & 264.33404 & -24.05816 & 20.8 & & & . \\
\hline $003.1+05.2$ & PHR1733-2327 & 4116647267584326528 & 0.80 & 263.27925 & -23.46682 & 19.3 & -2.952 & 1.038 & \\
\hline $003.1-02.1$ & PHR J1801-2718 & 4063170320596559488 & 0.95 & 270.35359 & -27.30307 & 19.4 & 0.879 & 1.137 & $3.91_{-140}^{+1.42}$ \\
\hline $003.2-04.4$ & KFL 12 & 4050866781253323264 & 0.99 & 272.62839 & -28.32308 & 17.6 & 0.211 & 0.115 & $15.77_{-569}^{+5.63}$ \\
\hline $003.2-06.2$ & M 2-36 & 4049788611227556864 & 0.76 & 274.42265 & -29.13886 & 17.0 & & & \\
\hline $003.3+66.1$ & SkAc 1 & 1227151802340968832 & 1.00 & 214.09141 & +13.87335 & 18.5 & 1.504 & 0.231 & $0.73_{-0.11}^{+0.16}$ \\
\hline $003.3-04.4$ & PPA J1810-2813 & 4050871767612742400 & 0.38 & 272.65051 & -28.22945 & 20.1 & & & \\
\hline $003.3-04.6$ & Ap 1-12 & 4050854450293755136 & 0.99 & 272.89623 & -28.37689 & 13.3 & 0.112 & 0.024 & $6.28^{+0.85}$ \\
\hline $003.3-07.5$ & KFL 19 & 4050998383326315264 & 0.99 & 275.78719 & -29.72386 & 17.7 & 0.070 & 0.111 & -0 \\
\hline $003.5+02.7$ & PHR J1743-2431 & 4068113170792152448 & 0.82 & 265.91340 & -24.53172 & 19.6 & 1.202 & 0.616 & \\
\hline $003.5+04.3$ & PPA J1737-2341 & 4116403961961160192 & 0.24 & 264.36853 & -23.69195 & 18.6 & 1.124 & 0.186 & \\
\hline $003.5-02.4$ & IC 4673 & 4063199904342190208 & 0.71 & 270.82691 & -27.10632 & 18.0 & 0.426 & 0.173 & $2.82_{-0.66}^{+0.76}$ \\
\hline $003.5-02.4 \mathrm{c}$ & IC4673a & 4063200729000157056 & 0.26 & 270.80096 & -27.08789 & 20.0 & 2.859 & 1.762 & -0. \\
\hline $003.5-02.9$ & MPA J1805-2721 & 4063136059789043968 & 0.21 & 271.30462 & -27.35236 & 16.6 & 0.096 & 0.065 & \\
\hline $003.5-04.6$ & NGC 6565 & 4050864449067582848 & 0.88 & 272.96868 & -28.17847 & 18.5 & -0.180 & 0.840 & $3.26_{-0.88}^{+0.90}$ \\
\hline $003.6+01.0$ & Kn 116 & 4067229442572424448 & 0.52 & 267.47417 & -25.24957 & 20.9 & -2.430 & 1.801 & \\
\hline $003.6+03.1$ & M 2-14 & 4068369945446105728 & 0.64 & 265.48858 & -24.18785 & 17.6 & -0.450 & 0.581 & $8.41_{-2.32}^{+2.36}$ \\
\hline $003.6+04.9$ & K 5-6 & 4116632733407368832 & 0.24 & 263.88016 & -23.19682 & 20.4 & & & \\
\hline $003.6-01.3$ & PHR J1759-2630 & 4064023026181980032 & 0.41 & 269.80029 & -26.50687 & 18.9 & 0.512 & 0.273 & $5.75_{-2.47}^{+2.61}$ \\
\hline $003.7-04.6$ & M 2-30 & 4050902790361481856 & 0.97 & 273.14330 & -27.96963 & 16.9 & & & $\ldots$ \\
\hline $003.8+00.3$ & PM 1-18 & 4067385946881204608 & 0.62 & 268.24725 & -25.45773 & 18.7 & -0.006 & 0.296 & \\
\hline $003.8+05.3$ & H 2-15 & 4116705060703628160 & 0.30 & 263.61155 & -22.88906 & 19.3 & 0.919 & 0.413 & $\cdots$ \\
\hline $003.8-04.3$ & H $1-59$ & 4050956223827379840 & 0.32 & 272.87211 & -27.77116 & 19.3 & & & \\
\hline $003.8-04.5$ & H $2-41$ & 4050910078792722816 & 0.54 & 273.09903 & -27.87069 & 18.3 & 0.329 & 0.280 & $6.80_{-2.04}^{+2.07}$ \\
\hline $003.8-17.1$ & $\mathrm{Hb} 8$ & 6756677421471688064 & 1.00 & 286.39974 & -33.19389 & 14.9 & $\ldots$ & $\ldots$ & \\
\hline $003.9+02.6$ & K 5-14 & 4068334275660862976 & 0.88 & 266.13575 & -24.22426 & 18.0 & & $\ldots$ & $\ldots$ \\
\hline $003.9-02.3$ & M 1-35 & 4063320541318246656 & 0.91 & 270.91374 & -26.72611 & 18.5 & & $\ldots$ & \\
\hline $003.9-03.1$ & KFL 7 & 4063052672857512448 & 0.91 & 271.70847 & -27.10543 & 19.4 & & & \\
\hline $003.9-14.9$ & $\mathrm{Hb} 7$ & 6759935304494857984 & 0.99 & 283.90749 & -32.26352 & 13.9 & 0.198 & 0.057 & $5.65_{-1.34}^{+1.84}$ \\
\hline $004.0+00.9$ & PN PM $1-182$ & 4067603001648428800 & 0.95 & 267.84518 & -25.03149 & 20.9 & & & $\ldots$ \\
\hline $004.0-02.5$ & PHR J1804-2643 & 4063311637847832576 & 0.26 & 271.24560 & -26.70976 & 17.7 & 0.131 & 0.127 & \\
\hline $004.0-02.6$ & PHR J1804-2645 & 4063311229824729984 & 0.26 & 271.24766 & -26.75472 & 17.1 & 0.321 & 0.168 & $5.56_{-186}^{+2.06}$ \\
\hline $004.0-03.0$ & M 2-29 & 4063244773875284224 & 0.75 & 271.67043 & -26.91567 & 14.4 & 0.053 & 0.038 & $8.77_{-1.51}^{+1.84}$ \\
\hline $004.0-04.6$ & Pa J1812.9-2741 & 4050916023029043968 & 0.48 & 273.24088 & -27.69907 & 19.4 & 0.650 & 0.415 & \\
\hline $004.0-05.8$ & Pe $1-12$ & 4052126172891146496 & 0.34 & 274.42632 & -28.28753 & 19.3 & 1.605 & 0.565 & $8.86_{-307}^{+2.84}$ \\
\hline $004.0-11.1$ & M 3-29 & 4047152806963235584 & 0.99 & 279.85756 & -30.67704 & 15.4 & 0.169 & 0.058 & $5.97_{-129}^{+1.61}$ \\
\hline $004.1+03.6$ & PPA J1741-2332 & 4116467566158377856 & 0.93 & 265.37005 & -23.53868 & 18.5 & -0.496 & 0.209 & \\
\hline $004.1+07.8$ & PHR J1726-2117 & 4120900483473337216 & 0.96 & 261.55122 & -21.29866 & 18.1 & -0.317 & 0.174 & \\
\hline
\end{tabular}


Table A.1: continued.

\begin{tabular}{|c|c|c|c|c|c|c|c|c|c|}
\hline PN G & PN name & Gaia source ID & reliability & $\begin{array}{c}\text { RA } \\
(\mathrm{deg})\end{array}$ & $\begin{array}{c}\text { Dec } \\
(\mathrm{deg})\end{array}$ & $\begin{array}{c}\mathrm{G} \\
(\mathrm{mag})\end{array}$ & $\begin{array}{c}\omega \\
(\mathrm{mas})\end{array}$ & $\begin{array}{c}\sigma_{\omega} \\
(\mathrm{mas})\end{array}$ & $\begin{array}{c}\text { distance } \\
(\mathrm{kpc})\end{array}$ \\
\hline $004.1-03.0$ & PHR J1806-2652 & 4063268348965374976 & 0.29 & 271.73353 & -26.88170 & 18.8 & 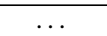 & & 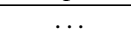 \\
\hline $004.1-03.8$ & KFL 11 & 4063000583418467968 & 0.64 & 272.55119 & -27.27644 & 19.8 & & $\ldots$ & 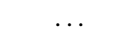 \\
\hline $004.2+01.5$ & K 6-29 & 4067690206718112512 & 0.83 & 267.41547 & -24.53705 & 20.9 & $\ldots$ & $\ldots$ & $\ldots$ \\
\hline $004.2+02.0 \mathrm{a}$ & MPA J1747-2414 & 4068463438357715456 & 0.47 & 266.90869 & -24.24058 & 20.9 & & & \\
\hline $004.2-03.2$ & KFL 10 & 4063077854365355776 & 0.59 & 272.00578 & -26.90061 & 17.2 & -0.104 & 0.109 & $14.26_{-343}^{+3.62}$ \\
\hline $004.2-04.3$ & H 1-60 & 4052470178293245312 & 0.44 & 273.10463 & -27.48700 & 16.4 & 0.283 & 0.082 & $5.59_{-191}^{+3.17}$ \\
\hline $004.2-05.9$ & M 2-37 & 4052151663642866048 & 1.00 & 274.65977 & -28.13295 & 17.3 & 0.286 & 0.151 & \\
\hline $004.3+01.8$ & H 2-24 & 4068460101018255744 & 0.41 & 267.15226 & -24.27618 & 16.2 & -0.024 & 0.142 & $5.58_{-119}^{+1.30}$ \\
\hline $004.3+02.1$ & K 5-17 & 4068510339301899264 & 0.31 & 266.88317 & -24.21879 & 19.7 & & & $\ldots$ \\
\hline $004.3+06.4$ & G $4.4+6.4$ & 4117845078118914048 & 1.00 & 262.96577 & -21.82180 & 19.3 & 1.262 & 0.377 & \\
\hline $004.3-01.4$ & PPA J1801-2553 & 4064173590585927296 & 0.99 & 270.32875 & -25.88918 & 18.7 & 0.057 & 0.307 & $\ldots$ \\
\hline $004.3-02.6$ & H $1-53$ & 4063339885864851072 & 0.34 & 271.48932 & -26.49504 & 18.9 & & & $\ldots$ \\
\hline $004.4+05.3$ & K 6-27 & 4117568654071497216 & 0.95 & 263.97380 & -22.33401 & 19.6 & 0.128 & 0.469 & \\
\hline $004.6+01.6$ & PHR1750-2406 & 4068481197909425536 & 0.23 & 267.53755 & -24.10447 & 19.3 & 0.335 & 0.286 & \\
\hline $004.6+06.0$ & H 1-24 & 4117684244606678400 & 0.94 & 263.40657 & -21.77358 & 17.6 & 0.048 & 0.150 & $6.34_{-153}^{+1.66}$ \\
\hline 004.6-09.9 & SB 9 & 4047457234247111936 & 0.99 & 278.92633 & -29.63972 & 19.4 & & & \\
\hline $004.7-05.5$ & SB 10 & 4052326176685965056 & 0.63 & 274.53691 & -27.53219 & 19.2 & 0.255 & 0.629 & $7.07_{-2.03}^{+2.04}$ \\
\hline $004.7-11.8$ & Hen 2-418 & 6737374120482481024 & 1.00 & 281.06080 & -30.32708 & 15.6 & 0.102 & 0.047 & $8.74_{-1.85}^{+2.03}$ \\
\hline $004.7-14.7$ & Fr $2-26$ & 6760041750949057536 & 0.60 & 284.09536 & -31.51318 & 17.6 & -6.967 & 0.692 & \\
\hline $004.8+02.0$ & H 2-25 & 4068552253876044416 & 0.50 & 267.25212 & -23.71524 & 16.1 & 0.169 & 0.052 & $6.96_{-198}^{+3.38}$ \\
\hline $004.8+05.7$ & PHR J1735-2146 & 4117672390472672128 & 0.38 & 263.82100 & -21.77507 & 20.8 & & & \\
\hline $004.8-05.0$ & M 3-26 & 4052445920552115200 & 0.80 & 274.04766 & -27.24941 & 17.8 & -0.194 & 0.151 & $7.52_{-166}^{+1.79}$ \\
\hline $004.8-22.7$ & Hen $2-436$ & 6744366945682210560 & 1.00 & 293.02795 & -34.21598 & 14.9 & -0.521 & 0.460 & $33.27_{-948}^{+9.51}$ \\
\hline $004.9+03.4$ & PHR J1744-2 & 4116889606820861312 & 0.47 & 266.07087 & -22.91592 & 20.7 & & & \\
\hline $004.9+04.9$ & M 1-25 & 4117211214062566528 & 0.95 & 264.62629 & -22.14418 & 16.6 & -0.515 & 0.157 & $7.43^{+1.58}$ \\
\hline $004.9-04.9$ & M 1-44 & 4052553741216415232 & 0.98 & 274.07229 & -27.07633 & 15.0 & 0.058 & 0.040 & $8.20_{-1.37}^{+1.58}$ \\
\hline $004.9-08.6$ & PPA J1831-2849 & 4048484315507463296 & 1.00 & 277.79654 & -28.82046 & 17.9 & -0.145 & 0.152 & $32.29_{-8.77}^{+8.92}$ \\
\hline $005.0+03.0$ & Pe $1-9$ & 4068846235848254208 & 0.20 & 266.40315 & -23.04064 & 19.0 & & $\ldots$ & $\ldots$ \\
\hline $005.0+04.4$ & H 1-27 & 4117160975714894848 & 0.56 & 265.07477 & -22.32173 & 17.0 & & 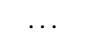 & $\ldots$ \\
\hline $005.0-03.9$ & H 2-42 & 4064555739648988160 & 0.89 & 273.09576 & -26.54848 & 19.0 & & & $\ldots$ \\
\hline $005.0-10.1$ & PPA J1837-2922 & 4047559656289337344 & 1.00 & 279.39606 & -29.37846 & 16.3 & -0.007 & 0.066 & $\ldots$ \\
\hline $005.0-14.6$ & Fr 2-27 & 6760328998365142400 & 1.00 & 284.11574 & -31.18873 & 17.4 & 0.061 & 0.090 & $\ldots$ \\
\hline $005.1+02.0$ & K 5-19 & 4068656505640715136 & 0.24 & 267.46392 & -23.46245 & 20.6 & & & $\ldots$ \\
\hline $005.1+03.2$ & PHR J1745-2254 & 4068851428434055040 & 0.59 & 266.32076 & -22.91619 & 18.4 & -0.098 & 0.175 & \\
\hline $005.1-03.0$ & H $1-58$ & 4064833739880077952 & 0.88 & 272.30763 & -26.04138 & 15.4 & -0.245 & 0.300 & $5.46_{-140}^{+1.48}$ \\
\hline $005.1-08.9$ & Hf $2-2$ & 4048497024309080064 & 0.99 & 278.12875 & -28.72233 & 17.2 & 0.284 & 0.086 & $4.00_{-0.83}^{+1.01}$ \\
\hline $005.2+04.2$ & M 3-13 & 4117171700355196672 & 0.58 & 265.40250 & -22.21750 & 16.8 & 0.143 & 0.143 & -0. \\
\hline $005.2+05.6$ & M 3-12 & 4118053199384406144 & 0.94 & 264.09431 & -21.52011 & 18.7 & -0.103 & 0.263 & $\ldots$ \\
\hline $005.2-02.4$ & PHR J180 & 4065636417718490240 & 0.81 & 271.70502 & -25.61261 & 17.6 & 0.715 & 0.130 & $\ldots$ \\
\hline $005.2-10.8$ & PPA J1840-2931 & 4047506471715127168 & 0.97 & 280.21118 & -29.52309 & 19.4 & -0.056 & 0.368 & $\ldots$ \\
\hline $005.3+02.5$ & MPA J1748-2259 & 4068882725801334144 & 0.44 & 267.05248 & -22.99410 & 18.6 & -0.014 & 0.252 & 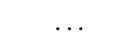 \\
\hline $005.3-02.0$ & PHR J1805-2520 & 4065659099458368384 & 0.81 & 271.36712 & -25.34069 & 20.4 & 0.543 & 0.933 & 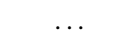 \\
\hline $005.4+04.0$ & PHR J1742-2214 & 4116986535735379200 & 0.72 & 265.72805 & -22.23781 & 20.0 & 6.184 & 1.429 & $\ldots$ \\
\hline $005.4+05.4$ & PHR J1737-2126 & 4118012895473955968 & 0.30 & 264.42300 & -21.44134 & 19.2 & 0.643 & 0.586 & $\ldots$ \\
\hline $005.4-01.9$ & PBOZ 34 & 4065731289318917632 & 0.96 & 271.35576 & -25.22663 & 20.4 & -0.530 & 1.154 & $\ldots$ \\
\hline $005.4-06.1$ & SB 12 & 4052306870817579520 & 0.96 & 275.47987 & -27.16296 & 18.3 & -0.429 & 0.252 & $\ldots$ \\
\hline $005.5+02.7$ & H 1-34 & 4068940935522394240 & 0.98 & 267.03194 & -22.77989 & 17.5 & -1.001 & 0.624 & 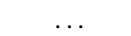 \\
\hline $005.5+06.1$ & M 3-11 & 4118277495405157632 & 0.85 & 263.83928 & -20.95652 & 16.6 & 0.014 & 0.095 & $\ldots$ \\
\hline $005.5-02.5$ & M 3-24 & 4065669098214601600 & 0.42 & 271.97463 & -25.40098 & 19.0 & 0.675 & 0.442 & $\ldots$ \\
\hline $005.5-04.0$ & H 2-44 & 4064670501058277504 & 0.21 & 273.41916 & -26.14434 & 19.3 & 0.608 & 0.429 &  \\
\hline $005.6-04.7$ & KFL 16 & 4052648539844689920 & 0.63 & 274.22507 & -26.38934 & 19.5 & & & $\ldots$ \\
\hline $005.7+04.5$ & PTB 5 & 4117204612583552256 & 0.93 & 265.41204 & -21.74268 & 19.1 & 0.272 & 0.272 & $\ldots$ \\
\hline $005.7-03.6$ & KFL 13 & 4064742892758691456 & 0.41 & 273.18613 & -25.73898 & 20.0 & & & $\ldots$ \\
\hline $005.7-05.3$ & M 2-38 & 4052583840315474560 & 0.92 & 274.85489 & -26.58878 & 18.8 & 0.302 & 0.290 & $\ldots$ \\
\hline $005.8+02.2 \mathrm{a}$ & MPA J17 & 4068900524147714688 & 0.20 & 267.58638 & -22.80679 & 17.5 & -0.158 & 0.122 & $\ldots$ \\
\hline $005.8+02.2 \mathrm{~b}$ & MPA J1750-2248b & 4068900631529845120 & 0.38 & 267.60978 & -22.81433 & 19.7 & 1.576 & 0.522 & 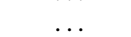 \\
\hline $005.8-09.2$ & PPA J1835-2811 & 4048631478276339840 & 1.00 & 278.83758 & -28.19793 & 18.6 & 0.694 & 0.201 & $\ldots$ \\
\hline $005.9+02.6$ & $\begin{array}{l}\text { MGE } \\
005.9143+02.6666\end{array}$ & 4068970931672721536 & 0.33 & 267.26817 & -22.49729 & 20.9 & 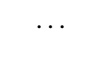 & 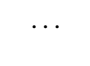 & $\ldots$ \\
\hline $005.9-04.2$ & Pa J1815.7-2551 & 4064703173079013632 & 0.93 & 273.94833 & -25.85449 & 19.1 & 0.561 & 0.476 & $\ldots$ \\
\hline $005.9-09.8$ & CGMW 4-2031 & 4048380549113958272 & 0.86 & 279.47782 & -28.45915 & 20.3 & -1.543 & 0.836 & \\
\hline $006.0+01.2$ & PHR J1754-2309 & 4070131259908465152 & 0.88 & 268.68663 & -23.15189 & 20.5 & -0.778 & 1.388 & $\ldots$ \\
\hline $006.0+02.8$ & Th $4-3$ & 4068994601118701312 & 0.32 & 267.15589 & -22.28033 & 16.1 & 0.064 & 0.065 & \\
\hline $006.0+02.9$ & MPA J1 & 4068998380797204224 & 0.23 & 267.10383 & -22.19774 & 19.2 & -0.100 & 0.360 & \\
\hline $006.0+05.6$ & PHR J1738-2052 & 4118196406426023168 & 0.35 & 264.60733 & -20.87156 & 20.2 & -2.209 & 1.396 & $\cdots$ \\
\hline $006.0-02.2$ & StDr102 & 4065796160446079104 & 0.72 & 272.01522 & -24.84988 & 18.0 & -0.047 & 0.145 & \\
\hline $006.0-03.6$ & M 2-31 & 4064771067972273920 & 0.77 & 273.31688 & -25.50148 & 17.6 & & & \\
\hline $006.0-41.9$ & PRMG 1 & 6776041195616496256 & 1.00 & 316.47322 & -37.14457 & 17.0 & 0.093 & 0.088 & $21.04_{-6.24}^{+6.34}$ \\
\hline $006.1+00.8$ & PPA J1756-2311 & 4069371359961206912 & 0.63 & 269.13818 & -23.19645 & 20.2 & 0.669 & 1.205 & $\ldots$ \\
\hline $006.1+03.5$ & PHR J1746-2150 & 4117103221360980608 & 0.40 & 266.55593 & -21.84711 & 20.0 & 1.512 & 0.694 & \\
\hline $006.1+08.3$ & M 1-20 & 4121750616461170432 & 0.95 & 262.24006 & -19.26504 & 16.7 & 0.707 & 0.482 & $7.59_{-2.42}^{+2.37}$ \\
\hline $006.2+01.0$ & HaTr 8 & 4069412793495388416 & 0.43 & 268.98296 & -22.98365 & 20.3 & 0.956 & 1.322 & $\ldots$ \\
\hline
\end{tabular}


Table A.1: continued.

\begin{tabular}{|c|c|c|c|c|c|c|c|c|c|}
\hline PN G & PN name & Gaia source ID & reliability & $\begin{array}{c}\text { RA } \\
(\mathrm{deg})\end{array}$ & $\begin{array}{c}\text { Dec } \\
(\mathrm{deg})\end{array}$ & $\begin{array}{c}\mathrm{G} \\
(\mathrm{mag})\end{array}$ & $\begin{array}{c}\omega \\
(\mathrm{mas})\end{array}$ & $\begin{array}{c}\sigma_{\omega} \\
\text { (mas) }\end{array}$ & $\begin{array}{c}\text { distance } \\
(\mathrm{kpc})\end{array}$ \\
\hline $006.2+06.9$ & PHR J1734-2000 & 4121407981117238400 & 0.98 & 263.55712 & -20.01407 & 19.8 & -0.208 & 0.436 & $\ldots$ \\
\hline $006.2-03.7$ & KFL 15 & 4064778734326138752 & 0.78 & 273.58114 & -25.34709 & 19.1 & 0.258 & 0.321 & \\
\hline $006.2-09.1$ & CGMW 4-1723 & 4048647245087934336 & 0.99 & 278.93592 & -27.85561 & 17.1 & 0.306 & 0.096 & $\ldots$ \\
\hline $006.3+02.2$ & MPA J1751-2223 & 4070418060644515072 & 0.37 & 267.91668 & -22.38827 & 19.4 & & & $\ldots$ \\
\hline $006.3+03.3$ & H 2-22 & 4117062676912301184 & 0.84 & 266.89142 & -21.78981 & 18.5 & -0.022 & 0.246 & $\ldots$ \\
\hline $006.3+04.4$ & H $2-18$ & 4117385040014126592 & 0.86 & 265.86981 & -21.16436 & 18.1 & 0.018 & 0.177 & $\ldots$ \\
\hline $006.3-04.6$ & PPA J1818-2541 & 4053063021354262912 & 0.57 & 274.50625 & -25.68436 & 19.7 & & $\ldots$ & . \\
\hline $006.4+02.0$ & M $1-31$ & 4070421084305148928 & 0.95 & 268.17269 & -22.36588 & 17.6 & $\ldots$ & . & $\ldots$ \\
\hline $006.4-04.6$ & Pe 2-13 & 4053063678434561664 & 0.24 & 274.55540 & -25.63570 & 19.9 & & & $\ldots$ \\
\hline $006.4-05.5$ & PHR J1821-2601 & 4053003445792400512 & 0.42 & 275.44230 & -26.02933 & 19.1 & 0.404 & 0.362 & $\ldots$ \\
\hline $006.5+03.4$ & PN PBOZ 29 & 4118615290396335744 & 0.95 & 266.95436 & -21.53958 & 19.2 & & & . \\
\hline $006.5+08.7$ & PHR J1728-1844 & 4122534632633972992 & 0.48 & 262.05849 & -18.74203 & 18.2 & 2.092 & 0.771 & $\ldots$ \\
\hline $006.6-01.1$ & MB 4475 & 4066158445235426048 & 0.97 & 271.31182 & -23.78590 & 20.3 & 1.543 & 1.955 & $\ldots$ \\
\hline $006.6-04.7$ & PHR J1818-2531 & 4053089242038392320 & 0.46 & 274.71148 & -25.52673 & 19.7 & & & $\ldots$ \\
\hline $006.7+03.2$ & MPA J1749-2127 & 4118575536092050176 & 0.23 & 267.26005 & -21.46871 & 20.5 & & & $\ldots$ \\
\hline $006.8+02.0$ & Pe 2-10 & 4070460529411078272 & 0.98 & 268.40458 & -21.97865 & 18.3 & -0.428 & 0.290 & \\
\hline $006.8+04.1$ & M 3-15 & 4118854193637373184 & 0.97 & 266.38213 & -20.96710 & 17.5 & 0.156 & 0.151 & $5.63_{-1.45}^{+1.59}$ \\
\hline $006.8-03.4$ & H 2-45 & 4065373776184045824 & 0.99 & 273.61977 & -24.72722 & 17.3 & 0.173 & 0.101 & $\ldots$ \\
\hline $006.8-07.5$ & PPA J1830-2637 & 4052020448007206144 & 0.73 & 277.61768 & -26.62201 & 18.5 & 0.436 & 0.234 & \\
\hline $006.8-08.6$ & $\mathrm{Al} 1$ & 4072763456463216384 & 0.72 & 278.73020 & -27.10533 & 17.4 & -0.041 & 0.103 & $9.41_{-200}^{+2.24}$ \\
\hline $006.9+01.5$ & MPA J1755-2212 & 4070344221607303296 & 0.43 & 268.90285 & -22.21332 & 21.2 & & & $\ldots$ \\
\hline $006.9+08.1$ & Kn J1731.5-1845 & 4121885822044187008 & 0.98 & 262.89834 & -18.75831 & 20.4 & -1.176 & 1.120 & $\ldots$ \\
\hline $006.9-05.1$ & MPA J1820-2524 & 4053176240876540544 & 0.89 & 275.24073 & -25.40625 & 18.1 & 0.187 & 0.189 & \\
\hline $006.9-30.2$ & Fr 2-29 & 6697958587097381248 & 1.00 & 302.40728 & -34.52542 & 12.5 & 2.877 & 0.040 & $\ldots$ \\
\hline $007.0+06.3$ & M 1-24 & 4120037195044273920 & 0.97 & 264.54833 & -19.62716 & 15.7 & 0.177 & 0.041 & $\ldots$ \\
\hline $007.0-06.0$ & H 1-66 & 4052929568083406464 & 0.86 & 276.23895 & -25.69852 & 19.7 & 1.269 & 0.874 & \\
\hline $007.0-06.8$ & Vy $2-1$ & 4052110122599119360 & 0.93 & 276.99843 & -26.11338 & 16.1 & 0.092 & 0.093 & $7.09_{-1.57}^{+1.73}$ \\
\hline $007.1+06.3$ & Kn 120 & 4120037985319136384 & 0.48 & 264.61871 & -19.54652 & 18.3 & 0.359 & 0.145 & $\ldots$ \\
\hline $007.1+07.4$ & РTB 7 & 4121634308764755328 & 1.00 & 263.62635 & -19.01695 & 19.8 & 1.392 & 1.550 & \\
\hline $007.2+03.4$ & PHR J1749-2101 & 4118662431936461824 & 0.85 & 267.30740 & -21.02886 & 20.2 & -1.439 & 0.759 & $\ldots$ \\
\hline $007.2+04.9$ & PHR J1743-2013 & 4119698137463144448 & 0.28 & 265.89147 & -20.23196 & 20.1 & -2.180 & 1.075 & $\ldots$ \\
\hline $007.2-08.1$ & PHR J1833-2632 & 4075841161280532352 & 0.62 & 278.33941 & -26.54100 & 19.3 & 0.854 & 0.341 & \\
\hline $007.3+03.5$ & РTB 10 & 4118664974555608576 & 0.53 & 267.36150 & -20.90897 & 19.7 & 1.428 & 0.567 & $\ldots$ \\
\hline $007.3+07.8$ & PHR J1733-1836 & 4121848266820444032 & 0.49 & 263.31627 & -18.60429 & 20.9 & & & $\ldots$ \\
\hline $007.3-10.1$ & PHR J1841-2716 & 4072602962097674112 & 0.95 & 280.47848 & -27.27855 & 18.4 & -0.328 & 0.294 & $\ldots$ \\
\hline $007.4+01.7$ & PHR J1755-2140 & 4070585839290632704 & 0.70 & 268.92682 & -21.67191 & 20.8 & & & $\ldots$ \\
\hline $007.4+02.5$ & Th 4-8 & 4070651775691846784 & 0.71 & 268.17793 & -21.26005 & 16.6 & 0.001 & 0.070 & $\ldots$ \\
\hline $007.4+03.1$ & $\mathrm{~Pa} 80$ & 4118707992955560448 & 0.22 & 267.67697 & -20.99284 & 21.0 & & & \\
\hline $007.4-01.8$ & PPA1809-2327 & 4066510117147168512 & 0.24 & 272.34146 & -23.46482 & 16.6 & 0.707 & 0.065 & $\ldots$ \\
\hline $007.5+07.4$ & M 1-22 & 4123340304074178432 & 0.81 & 263.79258 & -18.57242 & 19.3 & 0.886 & 0.335 & $\ldots$ \\
\hline $007.5-18.5$ & Kn 76 & 6758177189689878144 & 0.99 & 289.30990 & -30.46991 & 19.6 & 0.286 & 0.452 & 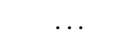 \\
\hline $007.6+06.9$ & M 1-23 & 4123152145849693696 & 0.96 & 264.34163 & -18.77827 & 18.6 & -0.119 & 0.257 & $\ldots$ \\
\hline $007.7+03.9$ & PHR J1748-2016 & 4118995686969542144 & 0.40 & 267.11395 & -20.27521 & 20.5 & & & \\
\hline $007.7-05.3$ & SB 14 & 4077240663477110912 & 0.99 & 275.92721 & -24.79114 & 16.5 & -0.119 & 0.087 & $\ldots$ \\
\hline $007.8+00.5$ & & 4069944927061605632 & 0.30 & 270.28069 & -21.93451 & 21.0 & & $\ldots$ & $\ldots$ \\
\hline $007.8-03.8$ & Sa 3-128 & 4065537362954637824 & 0.93 & 274.44795 & -24.04407 & 17.2 & & & \\
\hline $007.8-04.4$ & H 1-65 & 4065322923848085760 & 0.93 & 275.03684 & -24.25149 & 14.5 & 0.033 & 0.032 & $8.97_{-1.29}^{+1.46}$ \\
\hline $007.9+03.8$ & PHR J1748-2009 & 4118998057791972480 & 0.37 & 267.24271 & -20.15839 & 20.2 & -0.417 & 1.189 & -1 \\
\hline $007.9+04.3$ & PHR J1747-1957 & 4119065918329454080 & 0.88 & 266.81451 & -19.95778 & 19.6 & 0.375 & 0.486 & \\
\hline $007.9-12.5$ & PHR J1852-2749 & 4071869484740939520 & 1.00 & 283.21232 & -27.81904 & 19.0 & 0.124 & 0.269 & \\
\hline $008.0+03.9$ & NGC 6445 & 4119025030204237568 & 0.73 & 267.31262 & -20.00950 & 18.3 & 0.617 & 0.238 & $1.12_{-0.18}^{+0.18}$ \\
\hline $008.1-04.7$ & M 2-39 & 4089299595925528832 & 0.99 & 275.50484 & -24.17777 & 14.4 & 0.051 & 0.040 & $9.93_{-1.94}^{+2.26}$ \\
\hline $008.2+06.8$ & Hen 2-260 & 4123264059923436672 & 1.00 & 264.73904 & -18.29328 & 13.6 & 0.050 & 0.029 & $\begin{array}{l}12.07_{-2.63}^{+3.34} \\
1.63\end{array}$ \\
\hline $008.2-04.8$ & M 2-42 & 4089296950179444864 & 0.97 & 275.63368 & -24.15770 & 17.2 & 0.055 & 0.134 & $\ldots$ \\
\hline $008.3+09.6$ & РTB 26 & 4124562102059801216 & 1.00 & 262.30532 & -16.79546 & 16.6 & 0.055 & 0.062 & \\
\hline $008.3+14.8$ & $\mathrm{Kn} 41$ & 4139832699312612608 & 0.98 & 257.79189 & -13.95931 & 20.1 & -0.977 & 0.680 & $8.35_{-2.22}^{+2.27}$ \\
\hline $008.3-01.1$ & M 1-40 & 4069732858757201280 & 0.44 & 272.10812 & -22.28133 & 19.7 & & & $\ldots$ \\
\hline $008.3-05.3$ & PHR J18 & 4077291962704579584 & 1.00 & 276.18042 & -24.27525 & 18.6 & 0.175 & 0.196 & \\
\hline $008.4+08.8$ & IRAS 17292-1704 & 4124306804890009728 & 0.97 & 263.02432 & -17.11431 & 15.2 & 0.215 & 0.041 & . \\
\hline $008.4-02.8$ & PHR J1815-2300 & 4066445039812849408 & 0.45 & 273.80423 & -23.01804 & 19.9 & -0.232 & 0.962 & $\ldots$ \\
\hline $008.4-03.6$ & H 1-64 & 4089627525283072128 & 0.23 & 274.59929 & -23.41600 & 17.7 & 0.231 & 0.114 & $\ldots$ \\
\hline $008.6-02.2$ & PPA J1813-2233 & 4066661922835580288 & 0.31 & 273.29460 & -22.55767 & 19.3 & -0.119 & 0.523 & \\
\hline $008.6-02.6$ & $\mathrm{MaC} 1-11$ & 4090483254575155968 & 0.93 & 273.71210 & -22.73209 & 17.9 & 0.089 & 0.188 & $\ldots$ \\
\hline $008.8+01.6$ & PN PM 1-197 & 4071135492030995968 & 0.47 & 269.77093 & -20.45697 & 18.5 & 1.898 & 0.659 & \\
\hline $008.8+06.2$ & CBF 1 & 4123237018804802176 & 0.92 & 265.63518 & -18.16221 & 15.0 & 0.032 & 0.040 & $\ldots$ \\
\hline $008.9+03.7$ & PN PM 1-183 & 4119492494479957760 & 0.93 & 267.93718 & -19.39546 & 17.4 & -0.840 & 0.491 & 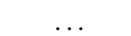 \\
\hline $008.9-05.3$ & $\mathrm{~Pa} 103$ & 4089418132675267456 & 0.33 & 276.52550 & -23.77796 & 15.7 & 0.098 & 0.050 & \\
\hline $009.0+04.1$ & Th 4-5 & 4120268165526590976 & 0.91 & 267.61814 & -19.05264 & 19.9 & 0.521 & 0.632 & $\ldots$ \\
\hline $009.0-02.4$ & PHR J1814-2220 & 4090688343507223040 & 0.83 & 273.73404 & -22.34339 & 19.7 & -0.227 & 0.469 & \\
\hline $009.1-02.7$ & PHR J1816-2222 & 4090520805520222208 & 0.26 & 274.08244 & -22.38043 & 18.8 & 0.010 & 0.228 & \\
\hline $009.3+02.5$ & Pre 14 & 4119279979437056000 & 0.67 & 269.21746 & -19.55022 & 20.9 & & & . \\
\hline $009.3+02.8$ & Th 4-9 & 4119330243430068480 & 0.90 & 269.00250 & -19.49078 & 17.1 & -0.087 & 0.154 & \\
\hline $009.3+04.1$ & Th 4-6 & 4120281909424954240 & 0.93 & 267.73847 & -18.78010 & 18.0 & 1.176 & 0.396 & \\
\hline
\end{tabular}


Table A.1: continued.

\begin{tabular}{|c|c|c|c|c|c|c|c|c|c|}
\hline PN G & PN name & Gaia source ID & reliability & $\begin{array}{c}\text { RA } \\
(\mathrm{deg})\end{array}$ & $\begin{array}{c}\text { Dec } \\
(\mathrm{deg})\end{array}$ & $\begin{array}{c}\mathrm{G} \\
(\mathrm{mag})\end{array}$ & $\begin{array}{c}\omega \\
(\mathrm{mas})\end{array}$ & $\begin{array}{c}\sigma_{\omega} \\
\text { (mas) }\end{array}$ & $\begin{array}{c}\text { distance } \\
(\mathrm{kpc})\end{array}$ \\
\hline $009.4+03.9$ & PTB 14 & 4119617048507979136 & 0.97 & 268.07425 & -18.86738 & 18.6 & -0.378 & 0.314 & \\
\hline $009.4-01.2$ & PHR J1811-2123 & 4093854116675316096 & 0.58 & 272.79436 & -21.38733 & 20.1 & & & 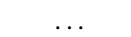 \\
\hline $009.4-03.6$ & Pre 35 & 4090538053989041024 & 0.38 & 275.05983 & -22.50408 & 16.2 & 0.035 & 0.066 & \\
\hline $009.4-05.5$ & NGC 6629 & 4089517157442187008 & 1.00 & 276.42689 & -23.20291 & 12.7 & 0.486 & 0.023 & $1.92_{-0.08}^{+0.09}$ \\
\hline $009.4-05.6$ & SB 16 & 4077444897868090496 & 0.65 & 277.08855 & -23.42388 & 19.9 & & & \\
\hline $009.4-06.6$ & PPA J1831-2356 & 4077538635493652224 & 1.00 & 277.98482 & -23.93550 & 18.4 & 0.308 & 0.242 & \\
\hline $009.4-09.8$ & M 3-32 & 4073399588285893888 & 0.95 & 281.17979 & -25.35942 & 17.5 & -0.089 & 0.146 & $8.08^{+1.91}$ \\
\hline $009.6+10.5$ & Abell 41 & 4136835641106850432 & 0.97 & 262.25843 & -15.21793 & 16.2 & 0.012 & 0.050 & $6.82_{-0.95}^{+1.05}$ \\
\hline $009.6+14.8$ & NGC 6309 & 4141505881131938560 & 1.00 & 258.51794 & -12.91051 & 16.1 & 0.383 & 0.064 & $2.58_{-0.34}^{+0.43}$ \\
\hline $009.6-10.6$ & M 3-33 & 4073217997070348032 & 1.00 & 282.05056 & -25.48125 & 15.8 & 0.164 & 0.054 & $6.41_{-145}^{+1.84}$ \\
\hline $009.7-03.9$ & PPA J1822-2223 & 4089816327689711104 & 0.97 & 275.53527 & -22.38545 & 15.7 & 0.018 & 0.040 & \\
\hline $009.8-04.6$ & H $1-67$ & 4090114299627717504 & 0.94 & 276.27067 & -22.58125 & 18.7 & 3.371 & 0.551 & $0.32_{-0.05}^{+0.08}$ \\
\hline $010.0-02.0$ & MPA J1815-2113 & 4090916943199396864 & 0.91 & 273.90406 & -21.22260 & 19.5 & 0.604 & 0.407 & \\
\hline $010.1+00.7$ & NGC 6537 & 4095023378533856256 & 0.44 & 271.30464 & -19.84325 & 16.8 & & & $\cdots$ \\
\hline $010.1+04.4$ & PTB 30 & 4120480332598055296 & 0.72 & 267.94361 & -18.06927 & 18.4 & 0.281 & 0.185 & \\
\hline $010.1+07.4$ & Sab 21 & 4124119475566179840 & 1.00 & 265.26954 & -16.41368 & 18.3 & 0.557 & 0.203 & $\ldots$ \\
\hline $010.4+00.9$ & IRAS 18021-1928 & 4095226925624354816 & 0.26 & 271.28812 & -19.46980 & 19.8 & -0.462 & 0.695 & $\ldots$ \\
\hline $010.4+04.5$ & M 2-17 & 4144530534542446976 & 0.75 & 268.02011 & -17.60170 & 18.7 & -0.781 & 0.337 & $\ldots$ \\
\hline $010.5+02.8$ & Pa J1758.5-1823 & 4143630382478662144 & 0.99 & 269.64786 & -18.39606 & 20.2 & & & . \\
\hline $010.6+02.4$ & MPA J1800-1834 & 4143424838162995328 & 0.74 & 270.03437 & -18.57609 & 19.5 & -0.160 & 0.440 & $\ldots$ \\
\hline $010.6+03.2$ & Th 4-10 & 4143700407610635904 & 0.80 & 269.27702 & -18.11139 & 17.4 & 0.320 & 0.165 & - \\
\hline $010.7+06.0$ & $\mathrm{~Pa} 79$ & 4123866283004347520 & 0.38 & 266.79525 & -16.66479 & 19.7 & -0.383 & 0.545 & $\ldots$ \\
\hline $010.7+07.4$ & Sa $2-230$ & 4124912429604974592 & 0.99 & 265.50840 & -15.93534 & 18.8 & 0.192 & 0.228 & \\
\hline $010.7-06.4$ & IC 4732 & 4077927519021635328 & 0.87 & 278.47764 & -22.64483 & 15.2 & & & \\
\hline $010.7-06.7$ & Pe 1-13 & 4077923838119276288 & 0.88 & 278.71543 & -22.72145 & 18.3 & -0.038 & 0.190 & \\
\hline $010.8-01.8$ & NGC 6578 & 4094354493205707392 & 0.98 & 274.06877 & -20.45079 & 15.4 & 0.371 & 0.040 & $2.47^{+0.25}$ \\
\hline $011.0+05.8$ & NGC 6439 & 4147847387468711040 & 0.80 & 267.08258 & -16.47905 & 18.4 & & & \\
\hline $011.0+06.2$ & M 2-15 & 4147907448379074304 & 0.96 & 266.72691 & -16.29027 & 18.4 & 0.105 & 0.259 & $7.19_{-1.94}^{+2.00}$ \\
\hline $011.0-02.9$ & PTB 31 & 4091282148480443648 & 0.99 & 275.22432 & -20.80343 & 19.4 & 0.680 & 0.363 & \\
\hline $011.0-05.1$ & M 1-47 & 4090264623539239936 & 0.97 & 277.29641 & -21.78171 & 15.4 & 0.163 & 0.048 & $6.23_{-1,32}^{+1.74}$ \\
\hline $011.1+11.5$ & M 2-13 & 4138416975017165440 & 0.95 & 262.14247 & -13.43910 & 18.2 & -0.820 & 0.378 & $10.46_{-2.66}^{+2.75}$ \\
\hline $011.1-07.9$ & SB 17 & 4079151550306831488 & 0.97 & 280.08303 & -22.90816 & 17.3 & 0.885 & 0.137 & $1.18_{-0.17}^{+0.256}$ \\
\hline $011.3+02.6$ & PN PM 1-199 & 4143751363058950144 & 0.97 & 270.17997 & -17.75533 & 19.3 & & & -0.1 \\
\hline $011.3+02.8$ & Th 4-11 & 4143767477777335296 & 0.49 & 270.03660 & -17.67872 & 14.8 & 0.244 & 0.043 & 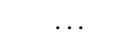 \\
\hline $011.3-09.1$ & РTB 32 & 4078149826461265920 & 1.00 & 281.29199 & -23.35920 & 18.4 & & & \\
\hline $011.3-09.4$ & My 121 & 4078224382749921024 & 0.99 & 281.64635 & -23.44676 & 13.2 & 0.212 & 0.039 & $4.36_{-0.63}^{+0.85}$ \\
\hline $011.4+17.9$ & DeHt 10 & 4335587859735196672 & 0.68 & 256.73139 & -9.78400 & 17.2 & 0.255 & 0.095 & $\ldots$ \\
\hline $011.4-07.3$ & SB 18 & 4079260195784967040 & 1.00 & 279.67933 & -22.40405 & 17.5 & -0.220 & 0.153 & \\
\hline $011.7+00.0$ & M 1-43 & 4095521422947121792 & 0.87 & 272.95371 & -18.77279 & 16.5 & 0.393 & 0.102 & $2.80_{-0.60}^{+0.87}$ \\
\hline $011.7-00.6$ & NGC 6567 & 4094749870714268544 & 1.00 & 273.43817 & -19.07615 & 14.2 & 0.350 & 0.035 & $2.63_{-022}^{+0.26}$ \\
\hline $011.7-06.6$ & M 1-55 & 4079529404360162176 & 0.98 & 279.14105 & -21.81744 & 13.9 & 0.093 & 0.036 & $7.76_{-1,35}^{+1.63}$ \\
\hline $011.7-07.0$ & Kn 77 & 4079460719236666624 & 0.87 & 279.50691 & -22.04692 & 14.5 & 0.123 & 0.037 & \\
\hline $011.7-41.3$ & Fr 2-46 & 6780966389233959168 & 1.00 & 316.44838 & -32.83117 & 13.4 & 2.312 & 0.046 &.. \\
\hline $011.8+03.7$ & PHR J1757-1649 & 4144686669576773888 & 0.99 & 269.41507 & -16.82213 & 15.6 & 0.563 & 0.034 & $\ldots$ \\
\hline $011.8-05.0$ & РTB 34 & 4091824315157913856 & 0.97 & 277.53314 & -21.08492 & 18.6 & 0.112 & 0.241 & \\
\hline $011.9+04.2$ & M $1-32$ & 4144898115027496320 & 0.97 & 269.08326 & -16.48449 & 15.6 & -0.013 & 0.049 & $5.82_{-0.69}^{+0.76}$ \\
\hline $011.9-07.4$ & $\mathrm{Kn} 78$ & 4079369008737614336 & 0.92 & 279.97521 & -22.05289 & 18.8 & 0.665 & 0.276 & $\ldots$ \\
\hline $012.0+07.4$ & PHR J1745-1453 & 4149099868648020864 & 0.99 & 266.28076 & -14.88976 & 19.7 & 0.595 & 0.405 & $\ldots$ \\
\hline $012.0-11.9$ & Kn 79 & 4075259652729340800 & 1.00 & 284.44326 & -23.82767 & 15.4 & 0.097 & 0.063 & $\ldots$ \\
\hline $012.1+01.1$ & [UHP2009] & 4095872442038835072 & 0.96 & 271.92305 & -17.85802 & 20.7 & & 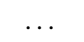 & $\ldots$ \\
\hline & G012.1175+01.1965 & & & & & & & & \\
\hline $012.1-02.1$ & PHR J1820-1926 & 4094535397244099840 & 0.80 & 275.07336 & -19.44323 & 20.3 & & & \\
\hline $012.1-11.2$ & CGMW 4-3783 & 4075514915539028224 & 1.00 & 283.77030 & -23.47025 & 15.3 & 0.152 & 0.034 & $\ldots$ \\
\hline $012.3-00.1$ & FBP2 & 4095675285854761472 & 0.21 & 273.30031 & -18.33547 & 20.0 & & & \\
\hline $012.4+02.4$ & MPA J1803-1657 & 4144211955433838592 & 0.97 & 270.97953 & -16.95618 & 20.4 & $\ldots$ & .. & .. \\
\hline $012.4+03.9$ & $\mathrm{~Pa} 84$ & 4145101906971129088 & 0.66 & 269.58410 & -16.20609 & 20.0 & & & \\
\hline $012.4+17.8$ & $\mathrm{~Pa} 12$ & 4335757356324800512 & 1.00 & 257.41095 & -9.01153 & 17.1 & 0.045 & 0.081 & \\
\hline $012.5+04.3$ & Sab 10 & 4145310023878030336 & 0.28 & 269.29378 & -15.93818 & 20.0 & 1.554 & 0.936 & $5.27_{-1.76}^{+1.72}$ \\
\hline $012.5-09.8$ & M 1-62 & 4078679481810532224 & 0.95 & 282.60852 & -22.57301 & 17.1 & 0.089 & 0.124 & $8.57_{-2.14}^{+1.29}$ \\
\hline $012.6-02.7$ & M 1-45 & 4096032936429271296 & 0.93 & 275.78326 & -19.28485 & 16.1 & 0.107 & 0.059 & $\ldots$ \\
\hline $012.8+09.6$ & $\mathrm{Kn} 42$ & 4161759920423904640 & 1.00 & 264.68630 & -12.95559 & 19.1 & 0.142 & 0.275 & \\
\hline $012.8-04.7$ & MPA J 1 & 4092038406321878784 & 0.94 & 277.84562 & -20.03561 & 16.6 & 0.148 & 0.071 & $\ldots$ \\
\hline $012.9+02.4$ & MPA J1804-1633 & 4144322280166446976 & 0.71 & 271.22049 & -16.56423 & 20.3 & & & $\ldots$ \\
\hline $012.9+02.6$ & MPA J1804-1627 & 4144373854134248960 & 0.68 & 271.04379 & -16.46242 & 18.3 & 1.237 & 0.378 & \\
\hline $012.9+03.3$ & PHR J1801-1602 & 4145164441661090944 & 0.47 & 270.39503 & -16.03876 & 21.0 & & & \\
\hline $012.9+06.6$ & BMP J1749-1429 & 4148751048613304192 & 0.83 & 267.41539 & -14.48836 & 20.4 & -1.497 & 0.901 & $\cdots$ \\
\hline $013.0-04.3$ & Pe 2-14 & 4092859608471896064 & 0.82 & 277.49779 & -19.67720 & 19.5 & & & \\
\hline $013.2-05.0$ & PHR J1832-1949 & 4092070227797242752 & 0.75 & 278.19129 & -19.82482 & 19.6 & 0.401 & 0.432 & \\
\hline $013.3+32.7$ & Sn 1 & 4407638066429175424 & 1.00 & 245.26831 & -0.26965 & 14.7 & -0.109 & 0.051 & $11.56_{-183}^{+1.98}$ \\
\hline $013.4+08.8$ & PHR J1742-1256 & 4161909453988004224 & 0.45 & 265.74159 & -12.94645 & 21.0 & & & \\
\hline $013.4-03.9$ & M 1-48 & 4093104043570072192 & 0.31 & 277.37523 & -19.09554 & 19.0 & 0.532 & 0.506 & $\cdots$ \\
\hline
\end{tabular}


Table A.1: continued.

\begin{tabular}{|c|c|c|c|c|c|c|c|c|c|}
\hline PN G & PN name & Gaia source ID & reliability & $\begin{array}{c}\text { RA } \\
\text { (deg) }\end{array}$ & $\begin{array}{l}\text { Dec } \\
\text { (deg) }\end{array}$ & $\begin{array}{c}\mathrm{G} \\
(\mathrm{mag})\end{array}$ & $\begin{array}{c}\omega \\
(\mathrm{mas})\end{array}$ & $\begin{array}{c}\sigma_{\omega} \\
(\mathrm{mas})\end{array}$ & $\begin{array}{c}\text { distance } \\
(\mathrm{kpc})\end{array}$ \\
\hline $013.4-04.2$ & V-V 3-4 & 4092899019087172608 & 0.90 & 277.64203 & -19.24602 & 19.4 & & & \\
\hline $013.7-04.7$ & РТВ 37 & 4092855824530810496 & 0.99 & 278.14434 & -19.23536 & 18.8 & 0.617 & 0.265 & \\
\hline $013.7-10.6$ & Y-C 2-32 & 4084782767771399040 & 1.00 & 283.87778 & -21.82776 & 16.0 & 0.084 & 0.067 & \\
\hline $013.8-02.8$ & SaWe 3 & 4096221021593115264 & 0.99 & 276.51532 & -18.20958 & 16.5 & 0.539 & 0.073 & $1.79_{-0.20}^{+0.24}$ \\
\hline $013.8-07.9$ & PC 21 & 4080123484141978752 & 1.00 & 281.39704 & -20.58324 & 17.7 & 0.370 & 0.131 & \\
\hline $014.0+04.8$ & РTB 19 & 4148581650775253120 & 0.80 & 269.60850 & -14.42324 & 18.5 & 0.154 & 0.198 & $4.83_{-1.24}^{+1.34}$ \\
\hline $014.0-02.5$ & $\operatorname{Tan} 2$ & 4096278711670069248 & 0.83 & 276.32128 & -17.94130 & 16.1 & 0.238 & 0.057 & \\
\hline $014.0-05.5$ & V-V 3-5 & 4092472408529260672 & 1.00 & 279.13475 & -19.32422 & 17.1 & 0.405 & 0.118 & $\ldots$ \\
\hline $014.1+02.7$ & Pa J1806.3-1520 & 4146705269757414272 & 0.93 & 271.58492 & -15.33544 & 19.1 & 0.102 & 0.319 & $\ldots$ \\
\hline $014.2+00.2$ & $\begin{array}{l}\text { MSX6C } \\
\text { G014.2361+00.2138 }\end{array}$ & 4097646121790662784 & 0.81 & 273.89353 & -16.47453 & 20.8 & & ( & $\cdots$ \\
\hline $014.2+03.8$ & PM 1-205 & 4146998736319570816 & 0.71 & 270.65964 & -14.70066 & 17.6 & -0.196 & 0.117 & \\
\hline $014.2+04.2$ & Sa 3-111 & 4147061236690945280 & 0.97 & 270.27752 & -14.50599 & 18.4 & 0.375 & 0.230 & $6.28_{-208}^{+2.13}$ \\
\hline $014.2-03.4$ & PTB 39 & 4093582365563693824 & 0.92 & 277.25165 & -18.17968 & 18.4 & 0.100 & 0.210 & \\
\hline $014.2-07.3$ & M 3-31 & 4080221267663409408 & 1.00 & 281.00739 & -19.91465 & 14.7 & & & $\ldots$ \\
\hline $014.3-07.2$ & PTB 40 & 4080225974947722752 & 1.00 & 280.91593 & -19.80906 & 17.3 & 0.024 & 0.100 & \\
\hline $014.4+06.9$ & Pa 81 & 4149764008058852352 & 0.58 & 267.97691 & -12.97115 & 20.1 & -0.031 & 0.709 & \\
\hline $014.4-06.1$ & SB 19 & 4092512742510443136 & 1.00 & 279.91691 & -19.23682 & 16.0 & 0.149 & 0.052 & $8.23_{-232}^{+3.14}$ \\
\hline $014.5-07.3$ & PN PM 1-254 & 4080250924463314944 & 0.97 & 281.12221 & -19.71255 & 17.4 & -0.061 & 0.093 & \\
\hline $014.7-11.8$ & SaWe 4 & 4081863633097152512 & 0.94 & 285.57341 & -21.44757 & 19.2 & 0.150 & 0.294 & $4.84_{-126}^{+1.32}$ \\
\hline $014.8-02.7$ & PTB 41 & 4096709995088279040 & 0.38 & 276.86088 & -17.40299 & 20.6 & & & \\
\hline $014.8-08.4$ & SB 20 & 4085994773196437888 & 1.00 & 282.35076 & -19.87038 & 15.0 & 0.360 & 0.052 & $\ldots$ \\
\hline $014.8-25.6$ & HaWe 14 & 6754240246564390784 & 1.00 & 299.55446 & -26.47115 & 18.5 & 1.528 & 0.233 & \\
\hline $014.9-03.1$ & SaSt 3-166 & 4096653198454533888 & 0.95 & 277.29693 & -17.45365 & 17.4 & 0.103 & 0.111 & . \\
\hline $015.1+02.8$ & MPA J1807-1425 & 4146916238566202368 & 0.82 & 271.91318 & -14.42486 & 19.9 & 0.195 & 0.631 & $\ldots$ \\
\hline $015.3-11.0$ & Kn 80 & 4084972983264857344 & 1.00 & 284.96750 & -20.58020 & 19.0 & 0.427 & 0.241 & \\
\hline $015.4+05.6$ & $\mathrm{~Pa} 44$ & 4150564551275781120 & 0.98 & 269.56681 & -12.79908 & 15.0 & 0.068 & 0.030 & \\
\hline $015.4-04.5$ & M 1-53 & 4093677434572665216 & 0.99 & 278.95146 & -17.60241 & 15.4 & 0.186 & 0.044 & $5.01_{-0.84}^{+1.10}$ \\
\hline $015.5+02.8$ & BMP J1808-1406 & 4146962654265880064 & 1.00 & 272.14622 & -14.11193 & 19.1 & 0.993 & 0.316 & $1.14_{-024}^{+0.28}$ \\
\hline $015.5-00.0$ & PHR J1818-1526 & 4098119397120573824 & 0.26 & 274.74713 & -15.43925 & 17.3 & & & $\ldots .24$ \\
\hline $015.8+02.7$ & PM 1-215 & 4147350232111461248 & 0.28 & 272.40249 & -13.80944 & 17.6 & 0.226 & 0.131 & - \\
\hline $015.9+03.3$ & M 1-39 & 4147559379838377344 & 0.97 & 271.87795 & -13.47995 & 16.5 & 0.279 & 0.074 & \\
\hline $016.0+13.5$ & Abell 42 & 4167707586821183360 & 1.00 & 262.87111 & -8.31945 & 19.8 & 0.289 & 0.385 & $2.71_{-068}^{+0.72}$ \\
\hline $016.0-04.3$ & M 1-54 & 4099764713235026688 & 1.00 & 279.03483 & -16.99918 & 19.6 & & & \\
\hline $016.0-07.6$ & SB 21 & 4098431349179300224 & 1.00 & 282.04611 & -18.49354 & 18.1 & 0.280 & 0.136 & $5.95_{-1.85}^{+2.09}$ \\
\hline $016.1+07.7$ & PTB 20 & 4163729038353275520 & 1.00 & 268.06256 & -11.17688 & 19.6 & & & $\ldots$ \\
\hline $016.1-04.7$ & M 1-56 & 4099723653388696320 & 0.91 & 279.44282 & -17.09631 & 16.2 & -0.307 & 0.221 & $\ldots$ \\
\hline $016.3-02.3$ & PHR J1828-1549 & 4103148941329438208 & 0.68 & 277.23240 & -15.82155 & 18.8 & 0.159 & 0.223 & \\
\hline $016.4+01.0$ & & 4146563226597613440 & 0.41 & 274.24909 & -14.16912 & 20.0 & 0.063 & 0.752 & \\
\hline $016.4-01.9$ & M 1-46 & 4103910524954236928 & 0.96 & 276.98466 & -15.54846 & 12.8 & 0.417 & 0.016 & $2.24_{-0.08}^{+0.08}$ \\
\hline $016.6-04.0$ & РTB 43 & 4102825336944868480 & 0.21 & 278.98311 & -16.35601 & 17.3 & 0.354 & 0.101 & $\ldots$ \\
\hline $016.6-10.5$ & Kn 55 & 4085678319975164928 & 0.38 & 285.14292 & -19.23700 & 19.7 & -0.141 & 0.398 & \\
\hline $016.7-07.3$ & SB 22 & 4098926576110700160 & 0.93 & 282.12683 & -17.73206 & 19.5 & -0.224 & 0.476 & $\ldots$ \\
\hline $016.8+07.0$ & PHR J17 & 4152110709452698240 & 0.67 & 269.08945 & -10.96016 & 20.9 & & & \\
\hline $016.8-01.7$ & BMP J1827-1504 & 4103988212315867008 & 0.83 & 276.96149 & -15.07340 & 19.2 & 0.347 & 0.340 & \\
\hline $016.9-02.0$ & Sa 3-134 & 4103960690161695232 & 0.94 & 277.33256 & -15.12776 & 18.1 & & & \\
\hline $016.9-09.7$ & РTB 44 & 4086522779285431680 & 1.00 & 284.41671 & -18.60463 & 16.3 & 0.334 & 0.055 & $3.14_{-049}^{+0.71}$ \\
\hline $017.0+11.1$ & GLMP 621 & 4165254065284620032 & 0.98 & 265.56022 & -8.72183 & 14.7 & 0.277 & 0.038 & $3.29_{-0.035}^{+0.49}$ \\
\hline $017.2-01.7$ & MPA J1828-1441 & 4104039335228566528 & 0.57 & 277.12742 & -14.68955 & 18.8 & -0.353 & 0.227 & \\
\hline $017.3-21.9$ & Abell 65 & 6864617817991978624 & 1.00 & 296.64254 & -23.13696 & 15.5 & 0.655 & 0.038 & $1.45_{-0.08}^{+0.08}$ \\
\hline $017.5+01.0$ & MPA J1819-1307 & 4152688193573412096 & 0.40 & 274.75948 & -13.11782 & 20.9 & & & \\
\hline $017.5-07.4$ & SB 23 & 4099164787867999104 & 0.64 & 282.55475 & -17.03928 & 18.2 & & & \\
\hline $017.5-09.2$ & SB 24 & 4086888981052416768 & 1.00 & 284.31887 & -17.84695 & 18.5 & 0.341 & 0.165 & \\
\hline $017.6-10.2$ & Abell 51 & 4086643583803222400 & 1.00 & 285.25584 & -18.20429 & 15.3 & 0.552 & 0.031 & $1.74_{-0.09}^{+0.10}$ \\
\hline $017.7-02.9$ & M 1-52 & 4103314314784098688 & 0.75 & 278.49388 & -14.87376 & 19.8 & & & \\
\hline $017.9-04.8$ & M 3-30 & 4100345805124045696 & 0.98 & 280.31204 & -15.56157 & 17.8 & 0.249 & 0.136 & $4.53_{-1.16}^{+1.34}$ \\
\hline $018.0+00.8$ & IRAS 18179-1249 & 4152816355413325440 & 0.99 & 275.18481 & -12.79799 & 18.3 & -0.040 & 0.219 & \\
\hline $018.0+20.1$ & $\mathrm{Na} 1$ & 4366642333006411264 & 1.00 & 258.21616 & -3.26665 & 16.6 & 0.204 & 0.063 & $5.14_{-101}^{+1.24}$ \\
\hline $018.2+01.6$ & DeGaPe 28 & 4153656760260335744 & 0.53 & 274.58319 & -12.24684 & 20.9 & & & \\
\hline $018.6-02.2$ & M 3-54 & 4104555933892707456 & 0.94 & 278.26565 & -13.73894 & 18.4 & 0.158 & 0.176 & $8.11^{+2.69}$ \\
\hline $018.8-01.6$ & MPA J1831-1319 & 4104803809373495296 & 0.25 & 277.83658 & -13.32734 & 18.5 & 0.239 & 0.199 & \\
\hline $018.8-01.9$ & РТВ 25 & 4104789412629934848 & 1.00 & 278.01896 & -13.43757 & 18.0 & 0.153 & 0.167 & $4.31_{-0.99}^{+1.09}$ \\
\hline $018.9+03.6$ & M 4-8 & 4157058752294046848 & 0.92 & 273.04001 & -10.71626 & 16.1 & 0.141 & 0.062 & \\
\hline $019.2-01.6$ & MPA J1831-1256 & 4104867439280324864 & 0.64 & 277.97089 & -12.93723 & 19.9 & 0.452 & 0.626 & \\
\hline $019.2-04.4$ & PM 1-251 & 4103595858442901888 & 0.65 & 280.60333 & -14.25308 & 17.2 & 0.289 & 0.089 & \\
\hline $019.4-01.6$ & Mul 14 & 4104964814776705280 & 0.82 & 278.15204 & -12.76724 & 18.5 & -0.209 & 0.231 & \\
\hline $019.4-13.6$ & DeHt 3 & 4084551148755614720 & 1.00 & 289.26713 & -18.02660 & 20.4 & 0.692 & 0.732 & $4.94_{-145}^{+1.45}$ \\
\hline $019.4-19.6$ & K 2-7 & 6868431267910764160 & 1.00 & 295.12304 & -20.45202 & 19.2 & 0.463 & 0.253 & $2.78+0.77$ \\
\hline $019.5-04.9$ & PHR J1844-1410 & 4105051680551805568 & 0.64 & 281.18233 & -14.18116 & 18.7 & 0.159 & 0.251 & \\
\hline $019.6+00.7$ & MPA J1824-1126 & 4154057188649467776 & 0.53 & 276.01665 & -11.43775 & 19.0 & -0.312 & 0.296 & $9.31_{-2.52}^{+2.62}$ \\
\hline
\end{tabular}


Table A.1: continued.

\begin{tabular}{|c|c|c|c|c|c|c|c|c|c|}
\hline PN G & PN name & Gaia source ID & reliability & $\begin{array}{c}\text { RA } \\
(\operatorname{deg})\end{array}$ & $\begin{array}{c}\text { Dec } \\
(\mathrm{deg})\end{array}$ & $\begin{array}{c}\mathrm{G} \\
(\mathrm{mag})\end{array}$ & $\begin{array}{c}\omega \\
(\mathrm{mas})\end{array}$ & $\begin{array}{c}\sigma_{\omega} \\
(\mathrm{mas})\end{array}$ & $\begin{array}{c}\text { distance } \\
(\mathrm{kpc})\end{array}$ \\
\hline $019.6+01.1$ & $\begin{array}{l}\text { MSX6C } \\
\text { G019.6095+01.1873 }\end{array}$ & 4154115428412694656 & 0.88 & 275.62519 & -11.27890 & 20.2 & & & \\
\hline $019.7-04.5$ & M 1-60 & 4105129677124985216 & 0.73 & 280.90872 & -13.74702 & 17.4 & & & \\
\hline $019.7-10.7$ & MPA J1906-1634 & 4088731114003376512 & 1.00 & 286.63663 & -16.56675 & 18.4 & 0.580 & 0.160 & $2.04_{-040}^{+0.48}$ \\
\hline $019.8+05.6$ & CTS 1 & 4158432626439950464 & 0.99 & 271.74918 & -8.92583 & 19.9 & & & \\
\hline $019.8-23.7$ & Abell 66 & 6864496115795567488 & 1.00 & 299.38126 & -21.61287 & 18.1 & 0.871 & 0.181 & $1.16_{-017}^{+0.20}$ \\
\hline $020.3-06.9$ & $\mathrm{~Pa} 120$ & 4101903087213994496 & 1.00 & 283.43106 & -14.33282 & 14.3 & 0.185 & 0.021 & $\ldots$ \\
\hline $020.4+02.0$ & MPA J1820-1009 & 4154521904099495296 & 0.98 & 275.21196 & -10.16191 & 20.6 & -2.879 & 1.690 & $\ldots$ \\
\hline $020.4-02.9$ & $\mathrm{~Pa} 111$ & 4106270248674345216 & 0.88 & 279.80578 & -12.42771 & 21.0 & $\ldots$ & & $\ldots$ \\
\hline $020.4-07.0$ & MPA J1854-1420 & 4101861447517419392 & 1.00 & 283.56140 & -14.33873 & 20.6 & & & \\
\hline $020.7-05.9$ & Sa $1-8$ & 4102207686291028864 & 0.99 & 282.68477 & -13.51734 & 14.3 & 0.251 & 0.026 & $3.55_{-0.31}^{+0.37}$ \\
\hline $020.7-08.0$ & MPA J1858-1430 & 4101453112078146432 & 1.00 & 284.58031 & -14.50719 & 16.4 & 0.725 & 0.063 & $1.37_{-0.11}^{+0.13}$ \\
\hline $020.9-01.1$ & M 1-51 & 4154696829542155392 & 1.00 & 278.37083 & -11.12401 & 18.0 & 0.339 & 0.156 & $2.54_{-0.01}^{+0.60}$ \\
\hline $020.9-11.3$ & PHR J1911-1546 & 4088900713689049728 & 1.00 & 287.76863 & -15.76875 & 17.5 & 0.535 & 0.130 & $2.05_{-0.35}^{+0.41}$ \\
\hline $021.0+03.4$ & MPA J1817-0856 & 4157866790265313152 & 0.93 & 274.27166 & -8.94718 & 18.0 & 0.669 & 0.622 & \\
\hline $021.0-04.1$ & PHR J1844-1226 & 4105553298336857600 & 1.00 & 281.03174 & -12.44726 & 18.1 & 0.186 & 0.136 & $11.78_{-369}^{+3.75}$ \\
\hline $021.1+00.4$ & GLMP 781 & 4155731538695389312 & 0.91 & 277.00548 & -10.23585 & 21.2 & & & $\ldots$ \\
\hline $021.2+02.9$ & MPA J1819-0901 & 4157853420013987072 & 0.71 & 274.77778 & -9.03074 & 18.7 & -0.005 & 0.293 & $\ldots$ \\
\hline $021.3+02.2$ & MPA J1822-0914 & 4159096181688213760 & 0.94 & 275.52381 & -9.23594 & 20.8 & & & . \\
\hline $021.4+02.5$ & $\mathrm{~Pa} J 1820.9-0901$ & 4159153218883234176 & 0.94 & 275.24726 & -9.02807 & 15.5 & 0.096 & 0.033 & $\ldots$ \\
\hline $022.0-03.1$ & M 1-58 & 4107013823419786624 & 0.85 & 280.73753 & -11.11482 & 18.7 & 0.469 & 0.292 & \\
\hline $022.0-04.3$ & AS 321 & 4106005536895223040 & 0.83 & 281.76660 & -11.68668 & 15.4 & 0.112 & 0.051 & $8.06_{-183}^{+2.22}$ \\
\hline $022.1-02.4$ & M 1-57 & 4107103777208876928 & 0.97 & 280.08438 & -10.66314 & 15.5 & 0.220 & 0.083 & $4.20_{-0.85}^{+1.83}$ \\
\hline $022.2+00.9$ & IRAS 18257-0908 & 4156149211359545984 & 0.81 & 277.12103 & -9.10333 & 20.3 & -1.713 & 1.121 & \\
\hline $022.5+01.0$ & $\mathrm{MaC} 1-13$ & 4156237687681900288 & 0.76 & 277.14698 & -8.72301 & 18.5 & 0.571 & 0.315 & $2.95_{-1.09}^{+1.31}$ \\
\hline $022.5+04.8$ & MA 2 & 4160551209235451904 & 0.89 & 273.80567 & -6.95350 & 18.5 & 0.215 & 0.269 & $\ldots$ \\
\hline $022.9+22.7$ & Alves 5 & 4387775530611235200 & 1.00 & 258.17347 & +1.92590 & 17.3 & 1.130 & 0.124 & 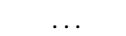 \\
\hline $023.0+04.3$ & MA 3 & 4160638521643128448 & 0.95 & 274.45584 & -6.80599 & 16.8 & 0.018 & 0.064 & $\ldots$ \\
\hline $023.1-02.5$ & Pre 48 & 4155290114832089728 & 0.88 & 280.69469 & -9.84667 & 19.8 & -0.147 & 0.534 & \\
\hline $023.2-02.6$ & MPA J18 & 4155290565857226112 & 0.98 & 280.74037 & -9.83066 & 18.6 & 0.220 & 0.216 & 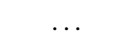 \\
\hline $023.8-01.7$ & K 3-11 & 4155606331827457408 & 0.39 & 280.28058 & -8.93316 & 16.3 & 0.200 & 0.064 & \\
\hline $023.8-06.2$ & BMP J1857-1054 & 4202075953819742080 & 1.00 & 284.29092 & -10.91419 & 19.0 & 0.722 & 0.240 & $1.91_{-0.48}^{+0.58}$ \\
\hline $023.9+01.2$ & MA 13 & 4159799662991309696 & 0.99 & 277.62663 & -7.46064 & 18.4 & 0.107 & 0.198 & \\
\hline $023.9-06.5$ & MPA J1858-1054 & 4202078904419339904 & 1.00 & 284.63659 & -10.90818 & 18.2 & 0.040 & 0.273 & $\ldots$ \\
\hline $024.0+05.7$ & Pa 97 & 4173439448308706944 & 0.99 & 273.64104 & -5.17117 & 20.3 & 0.083 & 1.255 & \\
\hline $024.1+03.8$ & M 2-40 & 4161093027951979776 & 0.98 & 275.34941 & -6.03218 & 16.1 & 0.128 & 0.053 & $5.97_{-1.05}^{+1.24}$ \\
\hline $024.2+05.9$ & M 4-9 & 4173456868697818880 & 0.85 & 273.57642 & -4.98916 & 19.7 & & & \\
\hline $024.2-05.2$ & M 4-11 & 4202377048194457344 & 0.89 & 283.57360 & -10.08686 & 18.0 & 0.347 & 0.159 & $3.57^{+0.98}$ \\
\hline $024.3-03.3$ & Pe $1-17$ & 4203399937651147520 & 0.30 & 281.95312 & -9.15219 & 20.7 & & & $\ldots$ \\
\hline $024.4+00.9$ & MPA J1832-0706 & 4159840031367633024 & 0.47 & 278.09532 & -7.11601 & 19.3 & 0.662 & 0.432 & \\
\hline $024.7+03.9$ & PHR J1822-0529 & 4161218617089810816 & 0.60 & 275.61315 & -5.48766 & 20.6 & -0.179 & 1.768 & $\ldots$ \\
\hline $024.8-01.4$ & Sab 8 & 4252568654478440064 & 0.20 & 280.44218 & -7.85822 & 19.5 & 0.777 & 0.393 & $\ldots$ \\
\hline $024.8-02.7$ & M 2-46 & 4251616408769092352 & 0.93 & 281.64423 & -8.46726 & 16.7 & 0.117 & 0.078 & \\
\hline $025.0-11.6$ & Abell 60 & 4186077574260953984 & 1.00 & 289.82413 & -12.24337 & 18.5 & 0.264 & 0.218 & $3.81_{-0.93}^{+1.01}$ \\
\hline $025.1-04.6$ & $\mathrm{~Pa} 121$ & 4203637225993816576 & 1.00 & 283.51906 & -9.02994 & 15.3 & 0.011 & 0.030 & $\ldots$ \\
\hline $025.1-08.4$ & MPA J1907-1043 & 4199466808402502656 & 1.00 & 286.88527 & -10.72920 & 18.3 & 0.032 & 0.180 & \\
\hline $025.3+40.8$ & IC 4593 & 4457218245479455744 & 1.00 & 242.93557 & +12.07140 & 11.2 & 0.376 & 0.045 & $2.38_{-023}^{+0.27}$ \\
\hline $025.3-04.6$ & K 4-8 & 4203838127400757248 & 0.99 & 283.58342 & -8.79257 & 16.2 & & & \\
\hline $025.4-04.7$ & IC 1295 & 4203649492421426816 & 0.98 & 283.65478 & -8.82707 & 16.8 & 0.649 & 0.064 & $1.54_{-0.13}^{+0.15}$ \\
\hline $025.4-16.4$ & Kn 56 & 4183190948217334400 & 1.00 & 294.43240 & -13.85566 & 20.3 & 1.203 & 0.633 & $\ldots$ \\
\hline $025.5-06.8$ & MPA J 1 & 4202650994066662656 & 1.00 & 285.65616 & -9.66832 & 17.4 & 0.178 & 0.091 & \\
\hline $025.6+02.8$ & MPA J1827-0510 & 4257140836167961984 & 0.93 & 276.98914 & -5.17308 & 18.7 & 0.122 & 0.280 & $\ldots$ \\
\hline $025.6-03.6$ & PM 1-259 & 4251974334170636416 & 0.79 & 282.76557 & -8.12223 & 14.5 & -0.000 & 0.033 & \\
\hline $025.8-17.9$ & NGC 6818 & 4182562817846811904 & 1.00 & 295.99048 & -14.15328 & 16.3 & 0.349 & 0.255 & $2.06_{-041}^{+0.46}$ \\
\hline $025.9+02.7$ & $\mathrm{~Pa}$ J1828.6-0457 & 4257162242283481088 & 0.92 & 277.17151 & -4.95999 & 19.6 & -0.709 & 0.435 & \\
\hline $025.9+10.3$ & MCS 1 & 4178943603525569280 & 1.00 & 270.46702 & -1.43850 & 16.5 & 0.124 & 0.081 & $8.13_{-2.00}^{+2.21}$ \\
\hline $025.9-02.1$ & Pe $1-15$ & 4252321092669536000 & 0.96 & 281.60246 & -7.24293 & 17.7 & 0.211 & 0.125 & $\ldots$ \\
\hline $026.0+46.6$ & WPS 4 & 1192780832638095872 & 1.00 & 237.72804 & +14.99269 & 14.9 & 0.315 & 0.038 & \\
\hline $026.0-09.4$ & PHR J1913-1021 & 4200764064607279616 & 0.42 & 288.27322 & -10.35289 & 20.3 & & & $\ldots$ \\
\hline $026.1-17.6$ & Pa 161 & 4183333506776770688 & 1.00 & 295.86910 & -13.74983 & 13.3 & 0.975 & 0.015 & $\ldots$ \\
\hline $026.2-03.4$ & PHR J1851-0732 & 4252076348209607296 & 0.75 & 282.88030 & -7.54148 & 18.3 & 0.225 & 0.205 & . \\
\hline $026.3-02.2$ & Pe $1-16$ & 4252368268565393920 & 0.71 & 281.88435 & -6.90114 & 19.3 & & . & $\ldots$ \\
\hline $026.4-01.7$ & PN PM 1-256 & 4253146413602687872 & 0.33 & 281.44335 & -6.54912 & 19.9 & & & $\ldots$ \\
\hline $026.5+02.0$ & Mul 13 & 4257218175601609984 & 0.98 & 278.13293 & -4.76982 & 18.0 & 0.225 & 0.145 & \\
\hline $026.5-03.0$ & Pe 1-19 & 4252307211263954688 & 0.99 & 282.43605 & -7.02649 & 15.9 & 0.229 & 0.064 & $\ldots$ \\
\hline $026.6-01.5$ & K 4-5 & 4253203278927003648 & 0.99 & 281.40255 & -6.30961 & 18.5 & 0.484 & 0.234 & . \\
\hline $026.8-08.5$ & Kn 65 & 4201133736742954880 & 1.00 & 287.71413 & -9.28030 & 18.6 & 0.203 & 0.210 & \\
\hline $026.9+04.4$ & FP J1824-0319 & 4269678120544342144 & 1.00 & 276.17028 & -3.33329 & 14.8 & 5.122 & 0.053 & $0.19_{-0.00}^{+0.00}$ \\
\hline $026.9-17.7$ & Kn J1945.0-1307 & 4183488121298349056 & 0.99 & 296.27049 & -13.12921 & 19.9 & -1.314 & 0.566 & $\ldots$ \\
\hline $027.3-01.1$ & Tan 1 & 4253538625585673344 & 0.25 & 281.36694 & -5.51719 & 17.9 & 0.139 & 0.129 & \\
\hline $027.4-03.5$ & Vy $1-4$ & 4253934209252777344 & 0.96 & 283.50786 & -6.43899 & 15.7 & 0.173 & 0.047 & $\ldots$ \\
\hline
\end{tabular}


Table A.1: continued.

\begin{tabular}{|c|c|c|c|c|c|c|c|c|c|}
\hline PN G & PN name & Gaia source ID & reliability & $\begin{array}{c}\text { RA } \\
(\operatorname{deg})\end{array}$ & $\begin{array}{c}\text { Dec } \\
(\mathrm{deg})\end{array}$ & $\begin{array}{c}\mathrm{G} \\
(\mathrm{mag})\end{array}$ & $\begin{array}{c}\omega \\
(\mathrm{mas})\end{array}$ & $\begin{array}{c}\sigma_{\omega} \\
(\mathrm{mas})\end{array}$ & $\begin{array}{c}\text { distance } \\
(\mathrm{kpc})\end{array}$ \\
\hline $027.5+01.0$ & PHR J1838-0417 & 4256963986573979136 & 0.86 & 279.50881 & -4.29007 & 20.7 & & & \\
\hline $027.6+04.2$ & M 2-43 & 4270080782317422208 & 1.00 & 276.66686 & -2.71597 & 16.3 & 0.147 & 0.224 & $6.24_{108}^{+2.14}$ \\
\hline $027.6+08.4$ & PN PM 1-217 & 4274355099417395072 & 1.00 & 272.91645 & -0.81726 & 17.3 & 0.025 & 0.110 & \\
\hline $027.6+10.5$ & Pre 21 & 4275388365469550720 & 0.98 & 271.08165 & +0.13460 & 20.0 & & & \\
\hline $027.6+16.9$ & DeHt 2 & 4376331092036268032 & 0.99 & 265.42040 & +3.11594 & 15.0 & 0.573 & 0.042 & $1.73_{-012}^{+0.14}$ \\
\hline $027.6-00.8$ & PHR J1844-0503 & 4253635490089186176 & 0.89 & 281.18983 & -5.06520 & 19.5 & & & \\
\hline $027.6-09.6$ & IC 4846 & 4201385082534035200 & 0.99 & 289.11759 & -9.04355 & 15.2 & -0.185 & 0.135 & $8.17_{-168}^{+1.79}$ \\
\hline $027.7+00.7$ & M 2-45 & 4256918322511326336 & 0.98 & 279.84101 & -4.33085 & 19.7 & -0.073 & 0.702 & $3.00_{-0.87}^{+0.68}$ \\
\hline $027.8+02.3$ & MPA J1833-0325 & 4258017864153209856 & 0.97 & 278.40966 & -3.43220 & 17.5 & 0.076 & 0.102 & \\
\hline $027.8-00.7$ & MPA J1844-0454 & 4253646519540446208 & 0.81 & 281.20681 & -4.90002 & 20.8 & & & 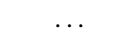 \\
\hline $027.8-13.7$ & Kn 57 & 4188025535210308864 & 0.48 & 292.90467 & -10.67821 & 20.1 & -0.169 & 0.822 & \\
\hline $027.9-01.4$ & PHR J1847-0507 & 4255077121584499072 & 0.94 & 281.86880 & -5.12334 & 13.8 & 0.255 & 0.019 & \\
\hline $028.0+10.2$ & WeSb 3 & 4275480995019447552 & 1.00 & 271.50320 & +0.37740 & 17.4 & 0.185 & 0.089 & $5.11_{-109}^{+1.26}$ \\
\hline $028.2-04.0$ & Pe $1-20$ & 4253881230894735616 & 0.82 & 284.32238 & -5.99783 & 20.4 & 0.201 & 1.280 & \\
\hline $028.5+01.6$ & M 2-44 & 4259355871048895232 & 0.21 & 279.40397 & -3.09920 & 18.7 & -0.058 & 0.300 & $3.68_{-0.91}^{+0.99}$ \\
\hline $028.5+05.1$ & K 3-2 & 4271262276290004864 & 0.99 & 276.25234 & -1.51460 & 17.1 & 0.127 & 0.086 & $7.58_{-1.92}^{+2.15}$ \\
\hline $028.7+02.7$ & K 3-7 & 4259650192241859840 & 0.95 & 278.55667 & -2.46024 & 19.0 & 0.824 & 0.282 & \\
\hline $028.7-03.9$ & Pe $1-21$ & 4254114494800691328 & 1.00 & 284.45713 & -5.46125 & 18.2 & 0.098 & 0.171 & $7.80_{-215}^{+2.28}$ \\
\hline $028.8-00.2$ & PHR J1845-0343 & 4258289069890784384 & 0.89 & 281.27570 & -3.72676 & 19.4 & 0.599 & 0.396 & \\
\hline $029.0+00.4$ & Abell 48 & 4258557110213184896 & 0.94 & 280.69550 & -3.22148 & 16.8 & 0.276 & 0.068 & $2.32_{-0.23}^{+0.26}$ \\
\hline $029.0+02.2$ & MPA J1836-0227 & 4259642190765140992 & 0.96 & 279.04459 & -2.45387 & 20.1 & & & \\
\hline $029.2-05.9$ & NGC 6751 & 4206136209740612352 & 1.00 & 286.48141 & -5.99232 & 13.7 & 0.291 & 0.045 & $2.96_{-0.31}^{+0.36}$ \\
\hline $029.3-01.2$ & PHR J1849-0347 & 4258331057475279616 & 0.25 & 282.31305 & -3.78312 & 16.6 & 0.745 & 0.058 & -0 \\
\hline $029.4-02.3$ & MPA J1853-0414 & 4255056711905102976 & 0.44 & 283.37791 & -4.23553 & 20.1 & 2.161 & 1.817 & \\
\hline $029.5-00.2$ & $\begin{array}{l}\text { IPHAS J184616.33- } \\
030626.0\end{array}$ & 4258616312027640448 & 0.70 & 281.56805 & -3.10724 & 20.2 & & & $\ldots$ \\
\hline $029.9+03.7$ & $\begin{array}{l}\text { IPHASX } \quad J 183249.6- \\
005638\end{array}$ & 4272105636060191232 & 0.99 & 278.20701 & -0.94402 & 19.3 & -1.241 & 0.589 & $\ldots$ \\
\hline $029.9+05.8$ & $\mathrm{~Pa} 34$ & 4273177483801926272 & 0.90 & 276.31368 & +0.03478 & 19.2 & 0.401 & 0.290 & $\ldots$ \\
\hline $030.0-04.2$ & PHR J1901-0429 & 4254656180365848448 & 0.98 & 285.31104 & -4.49257 & 19.9 & 0.175 & 0.571 & $\ldots$ \\
\hline $030.5+01.5$ & IRAS $18390-0128$ & 4260148618930827008 & 1.00 & 280.41847 & -1.42159 & 20.7 & & & . \\
\hline $030.5-18.4$ & Fr 2-42 & 4190499986125543296 & 1.00 & 298.40011 & -10.32486 & 16.4 & 7.663 & 0.061 & \\
\hline $030.6-16.4$ & $\mathrm{Fe} 4$ & 4193856211011004672 & 1.00 & 296.62850 & -9.35559 & 16.1 & 0.158 & 0.051 & $9.21_{-260}^{+3.23}$ \\
\hline $030.8+03.4 \mathrm{a}$ & $\begin{array}{l}\text { IPHAS } \\
001550.0\end{array}$ & 4272532521447280384 & 0.98 & 278.88046 & -0.26395 & 17.7 & 1.872 & 0.220 & \\
\hline $031.0+04.1$ & K 3-6 & 4273359521695801472 & 0.99 & 278.32290 & +0.19633 & 18.3 & & & \\
\hline $031.0-10.8$ & M 3-34 & 4207957000957467392 & 1.00 & 291.75790 & -6.58466 & 16.0 & 0.225 & 0.062 & $4.95_{-105}^{+1.36}$ \\
\hline $031.2+05.9$ & K 3-3 & 4276358851977975168 & 0.98 & 276.78884 & +1.24076 & 19.9 & -0.315 & 0.629 & $4.50_{-1.36}^{+1.43}$ \\
\hline $032.0+01.7$ & PHR J1843+0002 & 4272429678461294464 & 0.40 & 280.93832 & +0.03951 & & 0.450 & 0.926 & -1. \\
\hline $032.0-03.0$ & K 3-18 & 4261880968243745152 & 0.76 & 285.14510 & -2.19946 & 16.9 & 0.100 & 0.412 & \\
\hline $032.1+07.0$ & PC 19 & 4277099858391308800 & 0.99 & 276.18551 & +2.49109 & 16.9 & 0.033 & 0.140 & $8.71_{-220}^{+2.33}$ \\
\hline $032.1-06.2$ & FP J1912-0331 & 4260597185313614976 & 1.00 & 288.13102 & -3.52566 & 14.0 & 8.034 & 0.036 & \\
\hline $032.3-04.5$ & $\begin{array}{l}\text { IPHASX J190631.5- } \\
023236\end{array}$ & 4261710097262261248 & 0.26 & 286.63167 & -2.54307 & 19.6 & 0.540 & 0.537 & \\
\hline $032.5-00.4$ & MPA J1852-0033 & 4266 & 0.82 & 283.10643 & -0.55734 & 19.8 & -0.424 & 0.667 & $\ldots$ \\
\hline $032.5-03.2$ & K 3-20 & 4262011019866089856 & 0.98 & 285.54231 & -1.81264 & 15.7 & -0.062 & 0.057 & $\ldots$ \\
\hline $032.5-05.2$ & MPA J1909-0242 & 4261269291843584000 & 1.00 & 287.38185 & -2.70823 & 19.3 & 0.577 & 0.402 & $\ldots$ \\
\hline $032.6+00.7$ & PM 1-258 & 4266422531037747200 & 0.23 & 282.00490 & +0.08011 & 19.7 & 0.633 & 0.706 & $\ldots$ \\
\hline $032.9+07.8$ & K 3-1 & 4278089212706036736 & 0.97 & 275.84048 & +3.60772 & 18.5 & -0.277 & 0.263 & \\
\hline $033.0-05.3$ & Abell 55 & 4261355470375324672 & 1.00 & 287.60737 & -2.33964 & 19.7 & 0.687 & 0.690 & $2.99_{-0.91}^{+0.91}$ \\
\hline $033.1-06.3$ & NGC 6772 & 4261038467432411776 & 0.97 & 288.65144 & -2.70676 & 18.4 & 1.110 & 0.184 & $1.00_{-0.15}^{+0.20}$ \\
\hline $033.2-01.9$ & Sa 3-151 & 4592 & $0 \mathrm{c}$ & 284.7 & -0.54931 & 18.9 & & & \\
\hline $033.2-04.0$ & PHR J1906-0133 & 4262198413530027520 & 1.00 & 286.56370 & -1.55599 & 19.4 & 0.239 & 0.380 & $\ldots$ \\
\hline $033.3-05.0$ & Рa 36 & 4261389417811016064 & 0.97 & 287.53789 & -1.87491 & 19.3 & 0.554 & 0.372 & . \\
\hline $033.4-06.9$ & PHR J1917-0240 & 4261120724620894720 & 0.98 & 289.31903 & -2.67389 & 20.4 & 0.565 & 0.885 & $\ldots$ \\
\hline $033.6+06.6$ & $\mathrm{~Pa} 17$ & 4283305879985226240 & 1.00 & 277.29236 & +3.66387 & 19.5 & -0.150 & 0.356 & \\
\hline $033.7-02.0$ & CBSS 1 & 4265789624653994624 & 0.89 & 285.06730 & -0.24249 & 19.5 & -0.174 & 0.645 & $10.63_{-411}^{+4.29}$ \\
\hline $033.7-02.0 \mathrm{a}$ & PHR J1900-0014 & 4265769356705057792 & 030 & 285.01404 & -0.23392 & 20.2 & -0.688 & 1.063 & \\
\hline $033.8+01.5$ & PHR J1847+0132 & 4278692805923570048 & 0.76 & 281.93923 & +1.54642 & 19.0 & 0.507 & 0.305 & $\ldots$ \\
\hline $033.9+01.2$ & $\begin{array}{l}\text { IPHAS } \\
\text { J184853.00+012852.2 }\end{array}$ & 4278677447120276096 & 0.98 & 282.22080 & +1.48114 & 19.9 & 0.295 & 0.577 & \\
\hline $033.9-07.3$ & $\mathrm{~Pa} 37$ & 4214612997681357568 & 0.35 & 289.87223 & -2.49996 & 18.8 & -0.509 & 0.341 & \\
\hline $034.1-10.5$ & HaWe 13 & 4213334746695221888 & 0.99 & 292.78011 & -3.70899 & 16.7 & 0.526 & 0.069 & $1.97_{-0.24}^{+0.32}$ \\
\hline $034.3+06.2$ & K 3-5 & 4283434037501295744 & 0.99 & 277.94095 & +4.08589 & 17.7 & 0.352 & 0.103 & $4.46_{-1.54}^{+2.54}$ \\
\hline $034.5+07.7$ & $\mathrm{~Pa} 16$ & 4284399924112532608 & 1.00 & 276.64571 & +4.98488 & 19.6 & -1.093 & 0.375 & \\
\hline $034.5-06.7$ & NGC 6778 & 4262769064373735296 & 1.00 & 289.60396 & -1.59645 & 16.7 & 0.161 & 0.080 & $3.65_{-0.52}^{+0.58}$ \\
\hline $034.5-11.7$ & PM 1-308 & 4210278482327706496 & 1.00 & 294.07299 & -3.89032 & 13.1 & 0.106 & 0.016 & $7.36_{-0.81}^{+1.52}$ \\
\hline $034.6+11.8$ & NGC 6572 & 4472051344537031808 & 0.98 & 273.02632 & +6.85361 & 12.6 & 0.537 & 0.058 & $1.73_{-0.15}^{+0.11}$ \\
\hline $034.7-06.2$ & PHR J1917-0112 & 4262994116345873024 & 0.91 & 289.28121 & -1.20716 & 14.8 & 0.170 & 0.026 & $\cdots$ \\
\hline $035.1+03.4$ & $\mathrm{~Pa} J 1843.0+0334$ & 4280446153312965376 & 0.72 & 280.76552 & +3.57461 & 19.1 & 0.696 & 0.416 & \\
\hline $035.2+05.2$ & $\mathrm{~Pa} 10$ & 4283781551897715072 & 1.00 & 279.29429 & +4.47182 & 18.6 & 0.066 & 0.230 & $6.32_{-1.62}^{+1.69}$ \\
\hline
\end{tabular}


Table A.1: continued.

\begin{tabular}{|c|c|c|c|c|c|c|c|c|c|}
\hline PN G & PN name & Gaia source ID & reliability & $\begin{array}{c}\text { RA } \\
(\mathrm{deg})\end{array}$ & $\begin{array}{l}\text { Dec } \\
(\mathrm{deg})\end{array}$ & $\begin{array}{c}\mathrm{G} \\
(\mathrm{mag})\end{array}$ & $\begin{array}{c}\omega \\
(\mathrm{mas})\end{array}$ & $\begin{array}{c}\sigma_{\omega} \\
(\mathrm{mas})\end{array}$ & $\begin{array}{c}\text { distance } \\
(\mathrm{kpc})\end{array}$ \\
\hline $035.7+19.2$ & $\mathrm{~Pa} 157$ & 4489876627044035456 & 1.00 & 266.78544 & +11.00581 & 16.3 & 0.308 & 0.054 & \\
\hline $035.7-05.0$ & K 3-26 & 4264027902125405056 & 0.96 & 288.66317 & +0.22667 & 19.5 & 0.802 & 0.643 & \\
\hline $035.9-01.1$ & Sh $2-71$ & 4268419317167114240 & 1.00 & 285.49981 & +2.15448 & 19.9 & 0.826 & 0.698 & $1.35^{+0.44}$ \\
\hline $035.9-02.9$ & Pa 50 & 4267584719119811968 & 0.68 & 286.88096 & +1.30600 & 20.4 & 1.314 & 0.883 & \\
\hline $036.0+17.6$ & Abell 43 & 4488953930631143168 & 1.00 & 268.38446 & +10.62340 & 14.7 & 0.458 & 0.033 & $2.14^{+0.16}$ \\
\hline $036.1-57.1$ & NGC 7293 & 6628874205642084224 & 1.00 & 337.41079 & -20.83717 & 13.5 & 5.012 & 0.044 & $0.20^{+0.00}$ \\
\hline $037.3-07.1$ & MPA J1924+0038 & 4263701961311861760 & 1.00 & 291.24049 & +0.64418 & 19.1 & 0.002 & 0.437 & -0.00 \\
\hline $037.5-05.1$ & Abell 58 & 4264850714784262272 & 0.66 & 289.58558 & +1.78301 & 21.0 & $\ldots$ & $\ldots$ & $\ldots$ \\
\hline $037.6-04.7$ & BMP J1917+0200 & 4264872636281775488 & 0.62 & 289.28052 & +2.00283 & 20.6 & & & $\ldots$ \\
\hline $037.7-05.9$ & PHR J1921+0136 & 4264623145896445184 & 1.00 & 290.33705 & +1.60386 & 17.7 & 0.077 & 0.104 & $\cdots$ \\
\hline $037.7-06.0$ & MPA J1921+0132 & 4264619130140417664 & 0.99 & 290.43543 & +1.54450 & 19.0 & 0.162 & 0.270 & \\
\hline $037.7-34.5$ & NGC 7009 & 6889338034837425920 & 1.00 & 316.04510 & -11.36349 & 12.8 & 0.812 & 0.056 & $1.19_{-0.07}^{+0.08}$ \\
\hline $037.8-02.5$ & Pa 52 & 4269008140031070208 & 0.99 & 287.39542 & +3.16781 & 19.1 & -1.076 & 0.491 & \\
\hline $037.9-03.4$ & Abell 56 & 4268179207028750592 & 1.00 & 288.27536 & +2.87979 & 19.4 & 0.661 & 0.319 & $1.69^{+0.45}$ \\
\hline $038.1-25.4$ & Abell 70 & 6907822573352460032 & 1.00 & 307.88832 & -7.08838 & 17.7 & 0.254 & 0.121 & $5.45^{-2.03}$ \\
\hline $038.2+12.0$ & Cn 3-1 & 4482121324862387968 & 1.00 & 274.39207 & +10.15087 & 12.6 & 0.138 & 0.035 & $5.63_{-0.78}^{+0.68}$ \\
\hline $038.3-02.5$ & $\begin{array}{l}\text { IPHASX } \\
\text { J191027.4+034046 }\end{array}$ & 4269096822545006848 & 0.93 & 287.61423 & +3.67977 & 20.8 & $\ldots$ & $\ldots$ & $\begin{array}{l}-0.78 \\
\ldots\end{array}$ \\
\hline $038.4-03.3$ & K 4-19 & 4268281088010981248 & 0.76 & 288.34417 & +3.41671 & 14.5 & 0.176 & 0.023 & $\ldots$ \\
\hline $039.0-04.0$ & $\begin{array}{l}\text { IPHASX } \\
\text { J191716.4+033447 }\end{array}$ & 4292267621344388864 & 0.84 & 289.31829 & +3.57980 & 14.3 & 0.246 & 0.019 & $\cdots$ \\
\hline $039.1-02.2$ & PM 1-285 & 4293351602342300160 & 0.57 & 287.72234 & +4.45786 & 20.9 & & & \\
\hline $039.5-02.7$ & M 2-47 & 4293191412929039232 & 0.92 & 288.39393 & +4.63444 & 16.1 & 0.201 & 0.051 & $4.64_{-079}^{+1.01}$ \\
\hline $039.7+06.0$ & Pa 18 & 4287278827831316864 & 0.99 & 280.50488 & +8.77357 & 20.8 & & & \\
\hline $039.8-06.9$ & PHR J1928+0253 & 4289193352436323072 & 0.22 & 292.21383 & +2.89818 & 20.3 & 0.409 & 0.751 & $\ldots$ \\
\hline $040.1+03.2$ & $\begin{array}{l}\text { IPHAS } \\
\text { XJ185309.4+075241 }\end{array}$ & 4310230750781889920 & 0.97 & 283.28925 & +7.87799 & 20.0 & 0.228 & 0.487 & $\ldots$ \\
\hline $040.4-02.0$ & Pa 129 & 4293609094235062528 & 0.90 & 288.17879 & +5.76284 & 17.7 & 0.251 & 0.124 & \\
\hline $040.4-03.1$ & K 3-30 & 4293331879884359680 & 0.97 & 289.11540 & +5.22204 & 17.9 & -0.032 & 0.180 & $8.50_{-2.19}^{+2.31}$ \\
\hline $040.7+03.4$ & $\begin{array}{l}\text { IPHASX } \\
\text { J185322.1+083018 }\end{array}$ & 4310333898714839936 & 0.34 & 283.34223 & +8.50516 & 19.7 & -0.593 & 0.405 & \\
\hline $040.8-09.7$ & WHTZ 1 & 4289684146940013184 & 1.00 & 295.18265 & +2.50883 & 16.9 & 0.555 & 0.071 & $1.91_{-0.23}^{+0.29}$ \\
\hline $040.9+08.3$ & Kn 1 & 4480747347648736384 & 1.00 & 278.96424 & +10.95539 & 20.0 & 0.111 & 0.513 & \\
\hline $041.2-00.6$ & HaTr 14 & 4306360401133813888 & 0.35 & 287.30710 & +7.09562 & 20.4 & -1.583 & 1.597 & $12.12_{-352}^{+3.54}$ \\
\hline $041.4-09.6$ & $\mathrm{~Pa} 13$ & 4289787771600957824 & 1.00 & 295.33764 & +3.12162 & 16.6 & 0.119 & 0.058 & $\ldots^{-3}$ \\
\hline $041.5+01.7$ & PM 1-273 & 4310136536377607040 & 0.93 & 285.27384 & +8.42665 & 21.1 & & & \\
\hline $041.5-04.6$ & $\begin{array}{l}\text { IPHAS } \\
\text { J192348.79+052759.9 }\end{array}$ & 4293002400007096832 & 0.98 & 290.95331 & +5.46653 & 18.5 & 0.391 & 0.188 & $\cdots$ \\
\hline $041.8+04.4$ & K 3-15 & 4311505638850116096 & 0.99 & 282.92309 & +9.91454 & 15.8 & -0.480 & 0.258 & \\
\hline $041.8-02.9$ & NGC 6781 & 4294123077230164736 & 1.00 & 289.61703 & +6.53873 & 16.7 & 2.004 & 0.068 & $0.49_{-0.02}^{+0.02}$ \\
\hline $042.0+05.4$ & K 3-14 & 4503757445981896832 & 1.00 & 282.13670 & +10.59740 & 15.0 & 0.008 & 0.040 & -0.0 \\
\hline $042.0-02.2$ & Pa J1916.3+0702 & 4306174446223256960 & 0.84 & 289.09816 & +7.03988 & 17.5 & -0.069 & 0.344 & $\ldots$ \\
\hline $042.2+01.1$ & $\begin{array}{l}\text { IPHASX } \\
\text { J190417.9+084916 }\end{array}$ & 4307548389086262656 & 0.88 & 286.07461 & +8.82110 & 19.7 & 0.196 & 1.265 & $\cdots$ \\
\hline $042.5-14.5$ & NGC 6852 & 4237745794618477440 & 1.00 & 300.16337 & +1.72801 & 17.9 & 0.390 & 0.117 & $3.82_{-1.02}^{+1.27}$ \\
\hline $042.6+01.3$ & $\begin{array}{l}\text { IPHASX } \\
\text { J190432.9+091656 }\end{array}$ & 4310668974884821248 & 0.54 & 286.13751 & +9.28224 & 19.9 & 0.034 & 0.547 & \\
\hline $042.7+00.8$ & [GKF2010] MN102 & 4307651880622158208 & 0.25 & 286.64027 & +9.12240 & 19.9 & -0.686 & 0.487 & $\ldots$ \\
\hline $043.0+01.7$ & $\begin{array}{l}\text { IPHASX } \\
\text { J190340.7+094639 }\end{array}$ & 4310749346635888640 & 0.75 & 285.91976 & +9.77747 & 17.5 & 0.272 & 0.102 & . \\
\hline $043.1+03.8$ & M 1-65 & 4311868924367555200 & 0.89 & 284.14013 & +10.86929 & 14.5 & 0.152 & 0.022 & $5.61_{-0.60}^{+0.74}$ \\
\hline $043.1+37.7$ & NGC 6210 & 1299564195037054592 & 1.00 & 251.12297 & +23.79980 & 12.5 & 0.486 & 0.032 & $1.95_{-0.11}^{+0.13}$ \\
\hline $043.2-02.0$ & PM 2-40 & 4308012932750878720 & 0.99 & 289.46067 & +8.25231 & 15.5 & 0.515 & 0.190 & \\
\hline $043.3+02.2$ & PM 1-276 & 4311037792327567744 & 0.64 & 285.57416 & +10.29245 & 17.3 & 0.306 & 0.091 & $3.55_{-076}^{+0.99}$ \\
\hline $043.3+10.4$ & Kn 2 & 4508624498600685440 & 1.00 & 278.16668 & +13.96732 & 17.3 & 0.107 & 0.079 & $6.43_{-1.25}^{+0.36}$ \\
\hline $043.3+11.6$ & M 3-27 & 4509558434941251584 & 1.00 & 276.95109 & +14.48499 & 13.1 & 0.147 & 0.051 & \\
\hline $043.5-13.4$ & Abell 67 & 4241183550857632896 & 1.00 & 299.61257 & +3.04997 & 19.5 & 0.556 & 0.327 & $3.88_{-1.16}^{+1.19}$ \\
\hline $044.0+05.2$ & K 3-16 & 4504427250399940864 & 0.93 & 283.25663 & +12.26661 & 18.2 & 0.789 & 0.235 & $\ldots$ \\
\hline $044.1+01.5$ & PM 1-281 & 4312523473047613568 & 0.97 & 286.63392 & +10.72321 & 20.6 & & & \\
\hline $044.1+05.8$ & CTSS 2 & 4504643171308707072 & 0.99 & 282.69526 & +12.62494 & 16.5 & 0.114 & 0.050 & \\
\hline $044.3+10.4$ & We 3-1 & 4509807233807831936 & 1.00 & 278.51105 & +14.82205 & 15.2 & 0.313 & 0.079 & $2.48_{-031}^{+0.35}$ \\
\hline $044.3-04.1$ & $\begin{array}{l}\text { IPHAS } \\
\text { J192717.94+081429.4 }\end{array}$ & 4296182638654046592 & 1.00 & 291.82477 & +8.24147 & 17.6 & 0.347 & 0.093 & \\
\hline $044.3-05.6$ & K 3-36 & 4295152602412852480 & 1.00 & 293.16468 & +7.46442 & 17.1 & 0.178 & 0.092 & $7.54_{-209}^{+2.38}$ \\
\hline $044.4+03.1$ & $\begin{array}{l}\text { IPHASX } \\
\text { J190115.5+114150 }\end{array}$ & 4313447582543796480 & 0.45 & 285.31447 & +11.69761 & 20.3 & -0.108 & 1.063 & \\
\hline $044.6+00.4$ & IRAS $19089+1032$ & 4312377345401979008 & 0.63 & 287.82142 & +10.62615 & 19.1 & 0.072 & 0.361 & $\ldots$ \\
\hline $044.9+00.8$ & $\begin{array}{l}\text { IPHASX } \\
\text { J191022.1+110538 }\end{array}$ & 4312451631147640960 & 0.96 & 287.59210 & +11.09411 & 20.9 & & $\ldots$ & \\
\hline $044.9+02.5$ & $\begin{array}{l}\text { IPHAS } \\
\text { J190414.21+115206.6 }\end{array}$ & 4313495308224822528 & 1.00 & 286.05921 & +11.86848 & 21.0 & & . & $\ldots$ \\
\hline
\end{tabular}


Table A.1: continued.

\begin{tabular}{|c|c|c|c|c|c|c|c|c|c|}
\hline PN G & PN name & Gaia source ID & reliability & $\begin{array}{c}\text { RA } \\
(\mathrm{deg})\end{array}$ & $\begin{array}{c}\text { Dec } \\
(\mathrm{deg})\end{array}$ & $\begin{array}{c}\mathrm{G} \\
(\mathrm{mag})\end{array}$ & $\begin{array}{c}\omega \\
(\mathrm{mas})\end{array}$ & $\begin{array}{c}\sigma_{\omega} \\
(\mathrm{mas})\end{array}$ & $\begin{array}{c}\text { distance } \\
(\mathrm{kpc})\end{array}$ \\
\hline $045.0-12.4$ & WHTZ 3 & 4248349003725092608 & 0.99 & 299.49724 & +4.79214 & 20.1 & -0.617 & 0.694 & $4.74_{-1.23}^{+1.26}$ \\
\hline $045.1-20.9$ & DSH J2027.7+0042 & 4230632362398259712 & 1.00 & 306.93266 & +0.71174 & 13.0 & 1.447 & 0.018 & $\ldots$ \\
\hline $045.3+00.9$ & Pa 127 & 4312855667292920960 & 0.56 & 287.71269 & +11.49728 & 20.8 & $\ldots$ & $\cdots$ & $\ldots$ \\
\hline $045.4-02.7$ & Vy 2-2 & 4308704598615929728 & 0.99 & 291.09257 & +9.89893 & 12.8 & & & \\
\hline $045.6+24.3$ & K 1-14 & 4556040392088374912 & 1.00 & 265.65281 & +21.45011 & 16.1 & 0.561 & 0.121 & $2.54_{-0.68}^{+1.20}$ \\
\hline $045.7-04.5$ & NGC 6804 & 4296362443149857920 & 1.00 & 292.89639 & +9.22532 & 14.0 & 1.417 & 0.067 & $0.70_{-0.03}^{+0.83}$ \\
\hline $045.9-01.9$ & K 3-33 & 4309563832565090432 & 0.98 & 290.61110 & +10.68922 & 18.8 & 0.600 & 0.351 & \\
\hline $046.3-03.1$ & PB 9 & 4314739847970750976 & 0.98 & 291.93671 & +10.40573 & 17.3 & 0.070 & 0.088 & $5.38_{-0.99}^{+1.11}$ \\
\hline $046.4-04.1$ & NGC 6803 & 4314488781356419456 & 0.99 & 292.81867 & +10.05598 & 16.8 & 0.934 & 0.410 & $4.05_{-1.54}^{+1.50}$ \\
\hline $046.6-01.4$ & $\mathrm{~Pa} 131$ & 4315858566694277504 & 0.82 & 290.52500 & +11.54467 & 18.4 & -0.010 & 0.148 & \\
\hline $046.7-13.7$ & $\mathrm{~Pa} \mathbf{J} 2005.8+0535$ & 4248742109181848704 & 0.75 & 301.45527 & +5.58447 & 18.8 & 0.536 & 0.211 & \\
\hline $046.8+03.8$ & Sh 2-78 & 4506484097383382272 & 1.00 & 285.79198 & +14.11631 & 17.6 & 1.432 & 0.104 & $0.70_{-0.05}^{+0.05}$ \\
\hline $046.9+24.3$ & PaGo 2 & 4556431199748103936 & 1.00 & 266.06398 & +22.60756 & 17.1 & 1.685 & 0.069 & \\
\hline $047.0+42.4$ & Abell 39 & 1305573511415857536 & 1.00 & 246.89046 & +27.90927 & 15.6 & 0.850 & 0.042 & $1.19_{-0.06}^{+0.06}$ \\
\hline $047.1+03.9$ & $\begin{array}{l}\text { IPHASX } \\
\text { J190319.0+142524 }\end{array}$ & 4506633764087096704 & 1.00 & 285.82930 & +14.42347 & 20.6 & $\cdots$ & $\ldots$ & $\ldots$ \\
\hline $047.1-02.6$ & Fr 2-28 & 4314870419308920832 & 0.98 & 291.78462 & +11.38087 & 14.1 & 0.905 & 0.021 & \\
\hline $047.1-04.2$ & Abell 62 & 4314617943916816384 & 1.00 & 293.32452 & +10.61656 & 18.6 & 0.186 & 0.213 & $1.98_{-032}^{+0.36}$ \\
\hline $047.2+01.7$ & PM 1-286 & 4314096397510483456 & 0.88 & 287.89931 & +13.51980 & 18.3 & -0.427 & 0.327 & \\
\hline $048.0-02.3$ & PB 10 & 4315832144050466304 & 0.99 & 292.06002 & +12.32674 & 19.0 & 0.233 & 0.404 & $3.71_{-1.05}^{+1.11}$ \\
\hline $048.0-04.4$ & $\begin{array}{l}\text { IPHASX } \\
\text { J193532.1+112115 }\end{array}$ & 4315045722705910528 & 1.00 & 293.88379 & +11.35434 & 19.2 & 0.207 & 0.278 & \\
\hline $048.1+01.1$ & K 3-29 & 4320225109682166784 & 0.98 & 288.87737 & +14.06385 & 19.4 & & & \\
\hline $048.1-03.2$ & $\begin{array}{l}\text { IPHASX } \\
\text { J193127.0+115622 }\end{array}$ & 4315370731432156160 & 0.86 & 292.86256 & +11.94006 & 20.3 & 0.135 & 0.642 & . \\
\hline $048.2+01.9$ & $\begin{array}{l}\text { IPHASX } \\
\text { J191255.4+143248 }\end{array}$ & 4320501361909209728 & 0.46 & 288.23090 & +14.54679 & 21.1 & $\cdots$ & $\ldots$ & . \\
\hline $048.5+04.2$ & K 4-16 & 4512994821132127360 & 0.98 & 286.21425 & +15.79350 & 20.1 & & & \\
\hline $048.7+01.9$ & M 4-13 & 4320554035406988672 & 0.99 & 288.41008 & +14.98862 & 17.7 & -0.124 & 0.130 & $4.82_{-0.85}^{+0.93}$ \\
\hline $048.8+08.4$ & Kn 66 & 4511470416959624576 & 0.98 & 282.47857 & +17.95415 & 17.7 & -0.201 & 0.137 & \\
\hline $048.9+04.3$ & $\begin{array}{l}\text { IPHASX } \\
\text { J190512.4+161347 }\end{array}$ & 4513118103860843392 & 1.00 & 286.30206 & +16.22984 & 20.1 & 0.147 & 0.477 & .. \\
\hline $048.9+04.3 \mathrm{a}$ & Pa 19 & 4513118413125457792 & 1.00 & 286.28602 & +16.25572 & 16.9 & 0.129 & 0.061 & \\
\hline $049.4+02.4$ & Hen $2-428$ & 4513170261953875840 & 0.76 & 288.27270 & +15.77770 & 15.5 & 0.224 & 0.075 & $3.38_{-051}^{+0.59}$ \\
\hline $049.6-07.7$ & Pa 54 & 4303586921715070592 & 1.00 & 297.60195 & +11.04404 & 19.9 & 0.541 & 0.703 & $\ldots$ \\
\hline $050.0+01.7$ & $\begin{array}{l}\text { IPHAS } \\
\text { J191701.33+155947.8 }\end{array}$ & 4321039328043783040 & 0.94 & 289.25551 & +15.99662 & 17.2 & 0.024 & 0.080 & . \\
\hline $050.2-11.9$ & $\mathrm{~Pa} 144$ & 4299561781443125504 & 1.00 & 301.63254 & +9.43910 & 18.4 & 0.087 & 0.182 & \\
\hline $050.4+05.2$ & Abell 52 & 4514604677947726976 & 1.00 & 286.13473 & +17.95213 & 17.7 & 0.338 & 0.114 & $4.04_{-1.03}^{+1.25}$ \\
\hline $050.4-01.6$ & K 4-28 & 4321218380945757568 & 0.92 & 292.56936 & +14.78936 & 19.5 & -0.249 & 0.907 & $\ldots$ \\
\hline $050.8+02.3$ & $\begin{array}{l}\text { IPHAS } \\
\text { J191621.43+165638.9 }\end{array}$ & 4513368517631376128 & 1.00 & 289.08932 & +16.94411 & 21.3 & 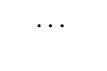 & $\ldots$ & . \\
\hline $051.0+03.0$ & Hen $2-430$ & 4513645762039397760 & 0.95 & 288.51722 & +17.52584 & 17.6 & -1.024 & 0.469 & $5.64_{-1.38}^{+1.45}$ \\
\hline $051.0-04.5$ & PC 22 & 4317178603426318720 & 1.00 & 295.51449 & +13.84372 & 18.0 & -0.104 & 0.143 & $6.18_{-1.24}^{+1.33}$ \\
\hline $051.3+01.8$ & PM 1-295 & 4514868732516293760 & 0.34 & 289.82793 & +17.19690 & 14.4 & 0.409 & 0.019 & \\
\hline $051.4+09.6$ & Hu 2-1 & 4530814090658118656 & 1.00 & 282.44817 & +20.84424 & 12.8 & 0.419 & 0.066 & $2.51_{-037}^{+0.51}$ \\
\hline $051.5+00.2$ & KLW 1 & 4322399045963388160 & 0.43 & 291.41868 & +16.55159 & 20.6 & -0.528 & 0.833 & $\ldots$ \\
\hline $051.5+05.1$ & Kn 4 & 4514801073882782336 & 1.00 & 286.81597 & +18.87788 & 18.0 & 0.046 & 0.118 & $\ldots$ \\
\hline $051.5+06.1$ & K 1-17 & 4517888747373052800 & 0.84 & 285.90567 & +19.35623 & 20.5 & $\ldots$ & $\ldots$ & $\ldots$ \\
\hline $051.8+01.7$ & IRAS $19183+1727$ & 4514907211117285504 & 0.97 & 290.13175 & +17.54692 & 21.1 & & & $\ldots$ \\
\hline $051.9-02.5$ & $\begin{array}{l}\text { IPHASX } \\
\text { J193633.5+153345 }\end{array}$ & 4318746640156604416 & 0.49 & 294.13987 & +15.56264 & 19.0 & 0.647 & 0.223 & \\
\hline $051.9-03.8$ & M 1-73 & 4318420669299100288 & 0.93 & 295.28872 & +14.94970 & 14.5 & 0.222 & 0.026 & $4.00_{-036}^{+0.43}$ \\
\hline $052.2+07.6$ & K 4-10 & 4518973106345424000 & 1.00 & 284.76565 & +20.61696 & 16.9 & -0.010 & 0.074 & $8.54_{-1.57}^{+1.70}$ \\
\hline $052.4-09.9$ & Kn 5 & 1805970255468857728 & 1.00 & 300.93443 & +12.37377 & 20.2 & 0.476 & 0.610 & \\
\hline $052.5-02.9$ & Me 1-1 & 4318785810234714752 & 0.98 & 294.79091 & +15.94673 & 12.5 & 0.270 & 0.020 & $3.70^{+0.30}$ \\
\hline $052.9+02.7$ & K 3-31 & 4515390553873264896 & 1.00 & 289.76102 & +19.03917 & 19.6 & & & \\
\hline $052.9-02.7$ & K 3-41 & 4318903049992391552 & 1.00 & 294.81614 & +16.34678 & 18.1 & 0.101 & 0.144 & $15.37_{-4.43}^{+4.50}$ \\
\hline $053.2-01.5$ & K 3-38 & 4322208834752173952 & 0.95 & 293.82640 & +17.21682 & & & & \\
\hline $053.3+03.0$ & Abell 59 & 4516549404740191488 & 0.90 & 289.66670 & +19.57574 & 20.6 & -0.791 & 1.140 & $2.00_{-0.60}^{+0.65}$ \\
\hline $053.3+24.0$ & Vy $1-2$ & 4584066702954631552 & 0.83 & 268.59602 & +27.99918 & 16.9 & & & \\
\hline $053.8-03.0$ & Abell 63 & 1820963913284517504 & 1.00 & 295.54286 & +17.08732 & 15.0 & 0.365 & 0.026 & $2.56_{-016}^{+0.18}$ \\
\hline $053.9-33.2$ & Fr 2-21 & 2691081097260836480 & 1.00 & 321.58819 & +0.97608 & 14.2 & 0.611 & 0.056 & \\
\hline $054.1-12.1$ & NGC 6891 & 1803234906762692736 & 1.00 & 303.78685 & +12.70431 & 12.3 & 0.388 & 0.032 & $2.42_{-0.17}^{+0.20}$ \\
\hline $054.2-03.4$ & $\begin{array}{l}\text { IPHASX } \\
\mathrm{J} 194359.5+170901\end{array}$ & 1820974088126464384 & 0.98 & 295.99796 & +17.15025 & 17.7 & 0.171 & 0.098 & \\
\hline $054.3-03.8$ & StDr 26 & 1820914366568717312 & 1.00 & 296.54551 & +17.10250 & 19.2 & 0.397 & 0.226 & $\cdots$ \\
\hline $054.4-02.5$ & M 1-72 & 1824067323576290304 & 1.00 & 295.39155 & +17.75485 & 15.0 & 0.485 & 0.345 & $\ldots$ \\
\hline $054.6+00.7 \mathrm{a}$ & StDr 85 & 2017614183546741120 & 0.93 & 292.45977 & +19.57229 & 20.0 & 0.171 & 0.593 & \\
\hline $055.0-15.1$ & Fr 2-15 & 1755635540865117568 & 1.00 & 306.80204 & +11.88552 & 13.7 & 0.653 & 0.030 & $\cdots$ \\
\hline $055.2+02.8$ & Hen 2-432 & 2018764096614237440 & 0.73 & 290.85335 & +21.13342 & 18.1 & 0.517 & 0.201 & \\
\hline
\end{tabular}


Table A.1: continued.

\begin{tabular}{|c|c|c|c|c|c|c|c|c|c|}
\hline PN G & PN name & Gaia source ID & reliability & $\begin{array}{c}\text { RA } \\
(\mathrm{deg})\end{array}$ & $\begin{array}{l}\text { Dec } \\
(\mathrm{deg})\end{array}$ & $\begin{array}{c}\mathrm{G} \\
(\mathrm{mag})\end{array}$ & $\begin{array}{c}\omega \\
(\mathrm{mas})\end{array}$ & $\begin{array}{c}\sigma_{\omega} \\
(\mathrm{mas})\end{array}$ & $\begin{array}{c}\text { distance } \\
(\mathrm{kpc})\end{array}$ \\
\hline $055.3+02.7$ & Hen 1-1 & 2018762967094831488 & 0.41 & 290.94546 & +21.11090 & 21.6 & & & \\
\hline $055.3+06.6$ & Abell 54 & 4521168453101490048 & 0.30 & 287.16495 & +22.98337 & & 0.521 & 0.744 & $4.63_{-1.41}^{+1.42}$ \\
\hline $055.4+16.0$ & Abell 46 & 4585381817643702528 & 0.99 & 277.82745 & +26.93670 & 15.0 & 0.404 & 0.029 & $2.43_{-0.16}^{+0.41}$ \\
\hline $055.5-00.5$ & M 1-71 & 1825515616543459584 & 0.92 & 294.11217 & +19.70667 & 17.4 & -0.342 & 0.393 & $3.26_{-0.76}^{+0.82}$ \\
\hline $055.6+02.1$ & Hen $1-2$ & 2018024881202947200 & 0.95 & 291.65725 & +21.15747 & 15.3 & 0.099 & 0.026 & $\begin{array}{l}7.02_{-0.90}^{+0.08} \\
+1.08\end{array}$ \\
\hline $056.0+02.0$ & K 3-35 & 2018131400729448960 & 0.96 & 291.93352 & +21.50113 & 20.5 & & & \\
\hline $056.1-03.8$ & RaMul 2 & 1821559921653783552 & 1.00 & 297.47376 & +18.67077 & 15.6 & 0.108 & 0.035 & $\ldots$ \\
\hline $056.4-00.9$ & K 3-42 & 1825749743804313600 & 0.98 & 294.89911 & +20.31717 & 20.1 & & 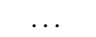 & $\ldots$ \\
\hline $056.6-00.8$ & StDr 30 & 1825965492917941376 & 0.47 & 294.94963 & +20.56945 & 21.1 & & & \\
\hline $056.8-06.9$ & K $3-51$ & 1821791540605697152 & 1.00 & 300.65142 & +17.61411 & 17.2 & 0.172 & 0.084 & $6.61_{-1.71}^{+2.06}$ \\
\hline $056.9-11.7$ & $\mathrm{~Pa} 26$ & 1805339376318567040 & 1.00 & 304.94233 & +15.23533 & 16.9 & -0.082 & 0.074 & \\
\hline $057.0+07.8$ & Pa 20 & 2035653626010277376 & 0.97 & 286.88256 & +24.99934 & 12.2 & 0.619 & 0.014 & \\
\hline $057.2-08.9$ & NGC 6879 & 1809552460055588608 & 1.00 & 302.61120 & +16.92255 & 14.7 & 0.125 & 0.040 & $6.90_{-124}^{+1.53}$ \\
\hline $057.6+01.8$ & PM 1-305 & 2019351991760108672 & 0.94 & 292.92205 & +22.72740 & 19.2 & 0.444 & 0.247 & \\
\hline $057.9+01.7$ & K 4-30 & 2019455895594998784 & 1.00 & 293.28760 & +22.97600 & 20.1 & 0.553 & 0.602 & \\
\hline $057.9-01.5$ & Hen 2-447 & 1827414443108647168 & 1.00 & 296.34234 & +21.33441 & 17.7 & 1.030 & 0.344 & $6.11_{-3.65}^{+2.98}$ \\
\hline $057.9-09.8$ & Alves 6 & 1809885066631267072 & 1.00 & 303.79164 & +17.05029 & 13.4 & 0.597 & 0.019 & $1.59_{-0.05}^{+0.05}$ \\
\hline $058.1-03.7$ & $\begin{array}{l}\text { IPHASX } \\
\text { J195343.7+202635 }\end{array}$ & 1826327227947840512 & 0.42 & 298.43216 & +20.44304 & 20.4 & -1.622 & 0.757 & \\
\hline $058.2+04.5$ & Kn 8 & 2023015633218874880 & 0.98 & 290.66130 & +24.55055 & 20.7 & 0.169 & 0.904 & $\ldots$ \\
\hline $058.3-02.9$ & $\begin{array}{l}\text { IPHAS } \\
\text { J195116.18+205954.2 }\end{array}$ & 1826560255678412672 & 1.00 & 297.81739 & +20.99838 & 20.2 & 0.035 & 0.629 & $\ldots$ \\
\hline $058.3-08.2$ & $\mathrm{~Pa} 23$ & 1810256976445430656 & 1.00 & 302.58938 & +18.19724 & 18.7 & 0.270 & 0.209 & \\
\hline $058.3-10.9$ & IC 4997 & 1808888702934248704 & 1.00 & 305.03648 & +16.73156 & 11.7 & 0.140 & 0.049 & $5.46_{-091}^{+1.10}$ \\
\hline $058.5-13.3$ & $\mathrm{~Pa} 146$ & 1805554841937332096 & 1.00 & 307.28922 & +15.61682 & 17.2 & 0.327 & 0.088 & \\
\hline $058.6+00.9$ & PM 1-309 & 2020219403339189760 & 0.99 & 294.37223 & +23.16293 & 21.3 & & & \\
\hline $058.6+06.1$ & Abell 57 & 2024098484670541952 & 0.95 & 289.27356 & +25.62577 & 17.5 & 0.470 & 0.074 & $2.31_{-0.36}^{+0.51}$ \\
\hline $058.6-03.6$ & V458 Vul & 1826449965231860608 & 1.00 & 298.60262 & +20.88113 & 18.1 & 0.193 & 0.113 & $9.70_{-2.89}^{+3.00}$ \\
\hline $058.6-05.5$ & WeSb 5 & 1823095492701560704 & 1.00 & 300.42505 & +19.91115 & 18.1 & & & $\ldots$ \\
\hline $058.8+07.4$ & $\operatorname{Dr} 24$ & 1822392423722386432 & 1.00 & 302.14089 & +19.02348 & 15.0 & 0.788 & 0.034 & . \\
\hline $058.8-16.9$ & Kn 121 & 1762118192700900736 & 1.00 & 310.50805 & +13.85416 & 15.6 & 0.783 & 0.043 & \\
\hline $058.9+01.3$ & K 3-40 & 2021005760309552256 & 0.93 & 294.09096 & +23.66323 & 17.6 & -0.318 & 0.087 & $9.32_{-149}^{+1.61}$ \\
\hline $058.9+09.0$ & Si $1-2$ & 2036937138334923648 & 1.00 & 286.52986 & +27.21621 & 19.1 & 0.498 & 0.201 & $4.88_{-1.61}^{+1.68}$ \\
\hline $059.0-01.7$ & Hen 1-3 & 1827574864387670400 & 1.00 & 297.10999 & +22.14368 & 19.1 & -0.272 & 0.273 & $8.05_{-2.09}^{-2.61}$ \\
\hline $059.1+03.3$ & $\begin{array}{l}\text { IPHASX } \\
\text { J192902.5+244646 }\end{array}$ & 2022884520783629312 & 0.99 & 292.26061 & +24.77961 & 19.9 & -0.459 & 0.430 & \\
\hline $059.1-00.7$ & Kn 9 & 1827853320031235712 & 1.00 & 296.24636 & +22.76318 & 16.2 & 2.550 & 0.045 & \\
\hline $059.2+01.0$ & Ou 3 & 2020279846433107200 & 0.96 & 294.57305 & +23.76350 & 20.5 & 0.476 & 0.948 & \\
\hline $059.4+02.3$ & K 3-37 & 2021325275814159360 & 0.99 & 293.44477 & +24.54081 & 19.8 & -1.360 & 0.453 & $9.84_{-2.58}^{+2.70}$ \\
\hline $059.7-00.8$ & $\begin{array}{l}\text { IPHASX } \\
\text { J194633.0+231659 }\end{array}$ & 2020051560320985856 & 0.77 & 296.63748 & +23.28316 & 15.5 & 0.309 & 0.034 & \\
\hline $059.7-01.0$ & $\begin{array}{l}\text { IPHASX } \\
\text { J194727.5+230816 }\end{array}$ & 1827892485806779776 & 0.46 & 296.86484 & +23.13783 & 19.2 & -0.366 & 0.279 & $\ldots$ \\
\hline $059.7-18.7$ & Abell 72 & 1761341417799128320 & 1.00 & 312.50856 & +13.55817 & 16.0 & 0.548 & 0.064 & $1.93_{-0.21}^{+0.26}$ \\
\hline $059.8-00.5$ & $\begin{array}{l}\text { 2MASS } \\
\mathrm{J} 19453289+2328105\end{array}$ & 2020070320738847232 & 0.99 & 296.38709 & +23.46956 & 20.8 & & (2. & $\ldots$ \\
\hline $059.9+00.6$ & PM 1-311 & 2020400444804204544 & 0.48 & 295.31917 & +24.22 & 20.6 & 1.188 & 0.926 & $\ldots$ \\
\hline $059.9+02.0$ & К 3-39 & 2021426048629219072 & 0.96 & 293.97681 & +24.91392 & 16.7 & 0.056 & 0.067 & $\ldots$ \\
\hline $060.0-04.3$ & Abell 68 & 1826936121144576896 & 1.00 & 300.04417 & +21.71553 & 18.8 & 0.482 & 0.187 & $\ldots$ \\
\hline $060.3-01.4$ & $\begin{array}{l}\text { IPHAS } \\
\text { J194957.59+232600.6 }\end{array}$ & 1828288000756444672 & 0.99 & 297.48992 & +23.43347 & 20.9 & 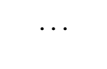 & 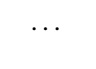 & $\ldots$ \\
\hline $060.3-02.0$ & $\begin{array}{l}\text { IPHAS } \\
\mathrm{J} 195224.67+230856.2\end{array}$ & 1828035525404444416 & 0.96 & 298.10283 & +23.14891 & 20.5 & 0.436 & 0.753 & \\
\hline $060.3-05.0$ & Kn 12 & 1823929193070538624 & 1.00 & 300.84391 & +21.59786 & 18.4 & 0.334 & 0.165 & \\
\hline $060.3-07.3$ & Hen 1-5 & 1828750899461025408 & 0.98 & 302.98357 & +20.33452 & 19.3 & 0.682 & 0.223 & $2.92_{-1.03}^{+1.23}$ \\
\hline $060.4+01.5$ & HuDo 1 & 2021265455564470144 & 0.41 & 294.71710 & +25.09233 & 17.0 & 0.006 & 0.077 & $18.21_{-4.95}^{+5.21}$ \\
\hline $060.5+01.8$ & Hen $2-440$ & 2021459171418312832 & 0.99 & 294.53482 & +25.26134 & 17.6 & 0.074 & 0.141 & \\
\hline $060.5+05.6$ & $\mathrm{~Pa} 21$ & 2025814135133944064 & 0.98 & 290.81254 & +27.12613 & 19.7 & 7.646 & 1.297 & $\ldots$ \\
\hline $060.5-05.8$ & Kn 13 & 1829680085557106176 & 0.89 & 301.70482 & +21.35701 & 20.0 & -0.487 & 0.440 & $\ldots$ \\
\hline $060.7-00.5$ & $\begin{array}{l}\text { IPHAS } \\
\text { J194727.51+241502.1 }\end{array}$ & 2020543724912372352 & 0.81 & 296.86454 & +24.25054 & 20.4 & 0.837 & 0.775 & \\
\hline $060.8-03.6$ & NGC 6853 & 1827256624493300096 & 1.00 & 299.90156 & +22.72121 & 14.0 & 2.570 & 0.037 & $0.39_{-0.01}^{+0.01}$ \\
\hline $060.8-07.9$ & $\mathrm{~Pa} 25$ & 1828581643391652992 & 1.00 & 303.75730 & +20.42257 & 18.3 & 0.120 & 0.138 & \\
\hline $060.9-00.5$ & IRAS & 2020643612977496704 & 0.92 & 297.05950 & +24.45749 & 18.6 & -0.479 & 0.253 & \\
\hline $061.0+08.0$ & K 3-27 & 2038257376937231488 & 1.00 & 288.62483 & +28.67924 & 17.3 & 0.125 & 0.063 & $7.55_{-1.52}^{+1.71}$ \\
\hline $061.2+04.0$ & StDr 86 & 2024908721680108672 & 1.00 & 292.74205 & +26.97875 & 21.0 & & & \\
\hline $061.4-09.5$ & NGC 6905 & 1816547660416810880 & 1.00 & 305.59577 & +20.10450 & 14.6 & 0.368 & 0.028 & $2.57_{-0.16}^{+0.18}$ \\
\hline $061.5-02.6$ & & 1834171384397003264 & 0.70 & 299.34681 & +23.88008 & 18.1 & 0.213 & 0.107 & \\
\hline $061.8+03.0$ & Pa 134 & 2025050524351107584 & 0.24 & 294.06395 & +26.95914 & 20.2 & 0.820 & 0.736 & \\
\hline $061.9+02.9$ & CGMW 5-11005 & 2025146800299621888 & 0.91 & 294.14962 & +27.06917 & 21.1 & $\cdots$ & & \\
\hline $061.9+41.3$ & DdDm 1 & 1331566790929938432 & 1.00 & 250.07562 & +38.70556 & 15.2 & 0.078 & 0.091 & $17.07_{-4.88}^{+5.02}$ \\
\hline
\end{tabular}


Table A.1: continued.

\begin{tabular}{|c|c|c|c|c|c|c|c|c|c|}
\hline PN G & PN name & Gaia source ID & reliability & $\begin{array}{c}\text { RA } \\
(\operatorname{deg})\end{array}$ & $\begin{array}{c}\text { Dec } \\
(\mathrm{deg})\end{array}$ & $\begin{array}{c}\mathrm{G} \\
(\mathrm{mag})\end{array}$ & $\begin{array}{c}\omega \\
(\mathrm{mas})\end{array}$ & $\begin{array}{c}\sigma_{\omega} \\
(\mathrm{mas})\end{array}$ & $\begin{array}{l}\text { distance } \\
(\mathrm{kpc})\end{array}$ \\
\hline $062.0+02.5$ & $\begin{array}{l}\text { IPHASX } \\
\text { J193827.8+265752 }\end{array}$ & 2025098146936552576 & 1.00 & 294.61613 & +26.96447 & 18.2 & 0.146 & 0.109 & $\ldots$ \\
\hline $062.1+03.1$ & $\begin{array}{l}\text { IPHASX } \\
\text { J193617.5+272051 }\end{array}$ & 2025166763313254400 & 1.00 & 294.07331 & +27.34781 & 20.2 & -0.605 & 0.491 & $\ldots$ \\
\hline $062.4+09.5$ & NGC 6765 & 2039515046435901440 & 1.00 & 287.77732 & +30.54545 & 17.6 & 0.276 & 0.078 & $3.87_{-0.74}^{+0.93}$ \\
\hline $062.4-00.2$ & M 2-48 & 2026955977938203264 & 0.97 & 297.61893 & +25.90850 & 22.0 & $\cdots$ & $\ldots$ & $\ldots$ \\
\hline $062.7+00.0$ & $\begin{array}{l}\text { IPHASX } \\
\text { J194940.9+261521 }\end{array}$ & 2027015592098419200 & 0.71 & 297.42040 & +26.25573 & 20.9 & $\ldots$ & $\ldots$ & $\ldots$ \\
\hline $062.7-00.7$ & $\begin{array}{l}\text { IPHASX } \\
\text { J195248.8+255359 }\end{array}$ & 2026853242333963904 & 0.84 & 298.20345 & +25.90007 & 21.0 & $\ldots$ & $\ldots$ & $\ldots$ \\
\hline $062.9-25.2$ & Fr 2-16 & 1746496503290277888 & 1.00 & 319.57805 & +12.02556 & 16.4 & 1.921 & 0.068 & \\
\hline $063.1+13.9$ & NGC 6720 & 2090486618786534784 & 1.00 & 283.39625 & +33.02915 & 15.6 & 1.270 & 0.044 & $0.78_{-0.03}^{+0.03}$ \\
\hline $063.2+02.3$ & Pa 53 & 2031177518533766016 & 0.83 & 295.45302 & +27.86731 & 17.1 & 0.094 & 0.058 & \\
\hline $063.2-04.9$ & Kn 14 & 1833636265835954048 & 0.99 & 302.42580 & +24.12752 & 20.0 & 0.195 & 0.407 & $\ldots$ \\
\hline $063.3+02.2$ & $\begin{array}{l}\text { IPHASX } \\
\text { J194240.5+275109 }\end{array}$ & 2031165664373595648 & 0.43 & 295.66939 & +27.85244 & 21.0 & & & . \\
\hline $063.3+15.8$ & PaStDr 5 & 2091480508577583488 & 1.00 & 281.42226 & +33.93456 & 16.7 & 1.674 & 0.048 & $\ldots$ \\
\hline $063.7+00.7$ & $\begin{array}{l}\text { IPHASX } \\
\text { J194930.9+273028 }\end{array}$ & 2027999723751718400 & 0.34 & 297.37876 & +27.50757 & 20.9 & $\ldots$ & $\ldots$ & $\ldots$ \\
\hline $063.8+00.1$ & K 3-48 & 2027934401557339520 & 0.78 & 298.03791 & +27.30853 & 18.9 & 0.475 & 0.179 & $\ldots$ \\
\hline $063.8-03.3$ & K 3-54 & 1834720521736114816 & 0.99 & 301.24430 & +25.44367 & 17.9 & -0.041 & 0.130 & \\
\hline $063.9-01.2$ & Te 1 & 2027268853416275456 & 1.00 & 299.34289 & +26.65228 & 20.1 & 0.860 & 0.425 & $1.71_{-046}^{+0.51}$ \\
\hline $064.1-04.9$ & $\begin{array}{l}\text { IPHASX } \\
\text { J201144.3+245006 }\end{array}$ & 1833780718479339264 & 0.96 & 302.93473 & +24.83512 & 19.8 & 0.333 & 0.329 & -0.40 \\
\hline $064.5+03.4$ & Kn 15 & 2031657180462828288 & 1.00 & 295.16806 & +29.50288 & 17.7 & 0.171 & 0.087 & $\because$ \\
\hline $064.5-06.9$ & Kn 16 & 1831949378782925952 & 0.99 & 305.04808 & +24.07696 & 20.7 & 0.478 & 1.175 & \\
\hline $064.6+48.2$ & NGC 6058 & 1380199049219990784 & 1.00 & 241.11060 & +40.68302 & 13.7 & 0.360 & 0.029 & $2.83_{-0.22}^{+0.26}$ \\
\hline $064.6-04.7$ & $\mathrm{~Pa} 145$ & 1835499250843571712 & 1.00 & 303.06571 & +25.37050 & 20.6 & 0.614 & 1.164 & \\
\hline $064.7+05.0$ & $\mathrm{BD}+303639$ & 2032744769234150016 & 1.00 & 293.68846 & +30.51634 & 10.3 & 0.618 & 0.032 & $1.58_{-008}^{+0.09}$ \\
\hline $064.9+15.5$ & M 1-64 & 2093171999835941504 & 1.00 & 282.50874 & +35.24322 & 19.3 & 0.797 & 0.248 & \\
\hline $064.9-02.1$ & K 3-53 & 1835203619659154688 & 0.99 & 300.84355 & +27.01520 & 18.4 & & & $\ldots$ \\
\hline $064.9-09.1 \mathrm{a}$ & $\mathrm{Pa} 15$ & 1830887113098897920 & 1.00 & 307.28160 & +23.18599 & 16.7 & 0.268 & 0.072 & $\ldots$ \\
\hline $065.2+02.2$ & $\mathrm{~Pa} 1$ & 2031795718947429248 & 0.94 & 296.76113 & +29.50702 & 21.3 & & & \\
\hline $065.2-05.6$ & Hen 1-6 & 1835268185902868992 & 0.84 & 304.33904 & +25.36308 & 20.6 & -1.878 & 1.152 & $3.78_{-1.01}^{+1.04}$ \\
\hline $065.4+03.1$ & TaWe 2 & 2032079697828332544 & 0.38 & 295.94960 & +30.23537 & 20.9 & 2.450 & 1.803 & $13.02_{-4.08}^{+4.05}$ \\
\hline $065.4+20.2$ & StDr 29 & 2096246444804803328 & 1.00 & 277.25865 & +37.33494 & 17.8 & 1.371 & 0.088 & $\ldots^{-4}$ \\
\hline $065.7+04.8$ & Kn 17 & 2033168076901487744 & 0.51 & 294.34508 & +31.24935 & 19.2 & 0.210 & 0.261 & \\
\hline $065.9+00.5$ & NGC 6842 & 2028777318992611072 & 1.00 & 298.75964 & +29.28828 & 16.1 & 0.456 & 0.038 & $2.11_{-0.15}^{+0.18}$ \\
\hline $066.5+15.1$ & Ra 1 & 2093708870751911040 & 1.00 & 283.69115 & +36.50334 & 18.7 & -0.042 & 0.131 & \\
\hline $066.5-14.8$ & Kn 45 & 1814597642173959168 & 1.00 & 313.26642 & +21.00303 & 18.5 & 0.484 & 0.175 & $2.72_{-063}^{+0.73}$ \\
\hline $066.7-28.2$ & NGC 7094 & 1770058865674512896 & 1.00 & 324.22072 & +12.78859 & 13.5 & 0.604 & 0.034 & $1.62_{-0.08}^{+0.09}$ \\
\hline $066.8+02.9$ & $\begin{array}{l}\text { IPHASX } \\
\text { J194751.9+311818 }\end{array}$ & 2033745393571783808 & 0.99 & 296.96630 & +31.30504 & 16.5 & 0.046 & 0.043 & $\cdots$ \\
\hline $066.9-05.2$ & Hen 1-7 & 1835909441720893952 & 0.99 & 304.90885 & +27.00310 & 17.8 & -0.094 & 0.141 & \\
\hline $066.9-07.8$ & Kn 19 & 1832550223218972800 & 1.00 & 307.33582 & +25.54421 & 19.9 & 0.176 & 0.468 & $3.60_{-0.95}^{+0.98}$ \\
\hline $067.5+02.8$ & Pa 137 & 2033791985369345152 & 0.98 & 297.47533 & +31.89961 & 21.0 & & & $\ldots$ \\
\hline $067.6+01.4$ & $\begin{array}{l}\text { IPHAS } \\
\text { J195549.80+311340.0 }\end{array}$ & 2033545522920043904 & 0.96 & 298.95744 & +31.22777 & 19.7 & -0.356 & 0.304 & te \\
\hline $067.9-00.2$ & K $3-52$ & 2030592853188209408 & 0.95 & 300.79761 & +30.54270 & 19.3 & & & \\
\hline $068.0+00.7$ & StDr 92 & 2030916109598082560 & 0.32 & 299.91612 & +31.23545 & 19.7 & 0.971 & 0.293 & \\
\hline $068.1+11.0$ & ETHOS 1 & 2050526964622031744 & 1.00 & 289.13119 & +36.16321 & 17.2 & 0.067 & 0.051 & $9.81_{-1.87}^{+2.07}$ \\
\hline $068.2+00.9$ & $\begin{array}{l}\text { IPHASX } \\
\text { J195919.0+312534 }\end{array}$ & 2030933937990031232 & 0.63 & 299.82948 & +31.42608 & 19.7 & 0.214 & 0.308 & -1. \\
\hline $068.2-16.0$ & $\mathrm{Kn} 44$ & 057377917056 & 1.00 & 315.33532 & +21.66836 & 18.2 & 0.318 & 0.152 & $\ldots$ \\
\hline $068.3+02.6$ & Ou 7 & 2033917432781399808 & 0.72 & 298.18896 & +32.42782 & 18.2 & 0.551 & 0.110 & \\
\hline $068.3-02.7$ & Hen $2-459$ & 1837452503203933184 & 0.98 & 303.49120 & +29.56548 & 15.8 & 0.991 & 0.300 & $1.83_{-0.83}^{+4.03}$ \\
\hline $068.6+01.1$ & Hen 1-4 & 2033978627428592000 & 0.90 & 299.82597 & +31.91056 & 20.4 & 0.257 & 0.663 & $2.31_{-0.69}^{+0.95}$ \\
\hline $068.6-00.2$ & $\begin{array}{l}\text { IPHASX } \\
\text { J200457.3+311416 }\end{array}$ & 2030777017063842176 & 0.99 & 301.24082 & +31.23820 & 19.0 & 0.059 & 0.193 & $a^{-0.09}$ \\
\hline 068.7 & K 4-41 & 0 & 1.00 & 2 & +3 & 18.7 & -0.047 & 0.206 & $9.98_{-3.07}^{+3.29}$ \\
\hline $068.7+14.8$ & Sp 4-1 & 2100120299148175872 & 0.99 & 285.11060 & +38.35201 & 15.7 & 0.090 & 0.081 & \\
\hline $069.2+01.2$ & KLW 5 & 2034099745530098432 & 0.89 & 300.17525 & +32.46142 & 20.5 & 0.704 & 0.706 & . \\
\hline $069.2+02.8$ & K 3-49 & 2035106004835235712 & 0.98 & 298.50255 & +33.37011 & 16.5 & -0.500 & 0.151 & \\
\hline $069.4-02.6$ & NGC 6894 & 2053683628140774528 & 0.99 & 304.09980 & +30.56501 & 18.2 & 0.686 & 0.113 & $1.51_{-0.20}^{+0.25}$ \\
\hline $069.6+15.7$ & Kn J1857.7+3931 & 2103243049609815296 & 1.00 & 284.42598 & +39.51670 & 15.6 & 0.447 & 0.037 & $\ldots$ \\
\hline $069.6-00.9$ & PM 1-319 & 2054027397321560960 & 0.99 & 302.65695 & +31.63247 & 21.2 & & & \\
\hline $070.0+01.8 \mathrm{a}$ & IRAS $19581+3320$ & 2034409361147890432 & 0.92 & 300.02871 & +33.48359 & 15.5 & 0.031 & 0.029 & \\
\hline $070.2+01.6$ & $\begin{array}{l}\text { 2MASS } \\
\mathrm{J} 20011443+3331220\end{array}$ & 2058239110989583744 & 0.92 & 300.31019 & +33.52279 & 20.8 & 1.453 & 1.160 & . \\
\hline $070.2+02.2$ & $\begin{array}{l}\text { IPHAS } \\
\text { J195912.66+335003.9 }\end{array}$ & 477359075364992 & 0.99 & 299.80275 & +33.83440 & 20.3 & -0.557 & 0.578 & $\ldots$ \\
\hline $070.2-08.7$ & Kn 21 & 1856444397601252224 & 1.00 & 310.32522 & +27.58500 & 19.0 & -0.040 & 0.227 & $\cdots$ \\
\hline
\end{tabular}


Table A.1: continued.

\begin{tabular}{|c|c|c|c|c|c|c|c|c|c|}
\hline PN G & PN name & Gaia source ID & reliability & $\begin{array}{c}\text { RA } \\
(\text { deg })\end{array}$ & $\begin{array}{c}\text { Dec } \\
(\mathrm{deg})\end{array}$ & $\begin{array}{c}\mathrm{G} \\
(\mathrm{mag})\end{array}$ & $\begin{array}{c}\omega \\
(\mathrm{mas})\end{array}$ & $\begin{array}{c}\sigma_{\omega} \\
(\mathrm{mas})\end{array}$ & $\begin{array}{l}\text { distance } \\
(\mathrm{kpc})\end{array}$ \\
\hline $070.5+11.0$ & Kn 61 & 2052811676760671872 & 1.00 & 290.41223 & +38.31588 & 18.2 & 0.139 & 0.111 & $5.88_{-124}^{+1.35}$ \\
\hline $070.9+02.2$ & PM 1-316 & 2058539140238094208 & 0.98 & 300.22048 & +34.47280 & 16.2 & 0.092 & 0.039 & -1 \\
\hline $071.3-00.6$ & $\begin{array}{l}\text { IPHASX } \\
\text { J201339.0+331507 }\end{array}$ & 2055401576400630528 & 0.96 & 303.41264 & +33.25194 & 16.5 & 0.229 & 0.038 & \\
\hline $071.3-05.8$ & $\mathrm{~Pa} 40$ & 1860817881199485696 & 1.00 & 308.40456 & +30.27254 & 20.7 & 1.491 & 0.928 & \\
\hline $071.6-02.3$ & M 3-35 & 2054325021390284032 & 1.00 & 305.26568 & +32.48998 & 16.6 & 1.005 & 0.307 & $2.20_{-100}^{+1.54}$ \\
\hline $072.0-24.6$ & $\mathrm{~Pa} 165$ & 1786272195418292992 & 1.00 & 324.72018 & +18.67099 & 12.9 & 0.699 & 0.040 & $\ldots$ \\
\hline $072.1+00.1$ & K 3-57 & 2055672679038891520 & 0.23 & 303.19919 & +34.34212 & 21.6 & & & $\ldots$ \\
\hline $072.3-05.3$ & Kn 23 & 1862554383714292608 & 0.55 & 308.60943 & +31.30901 & 20.3 & -0.398 & 0.605 & \\
\hline $072.7-17.1$ & Abell 74 & 1840395547924993152 & 1.00 & 319.21807 & +24.14751 & 17.0 & 1.520 & 0.086 & $0.67^{+0.04}$ \\
\hline $073.0+03.6$ & $\mathrm{NeVe} 2$ & 2060147622645380224 & 0.99 & 300.07781 & +36.99279 & 20.9 & -1.377 & 1.273 & \\
\hline $073.0-02.4$ & K 3-76 & 2055990571034870016 & 0.99 & 306.27029 & +33.58068 & 18.0 & 0.187 & 0.102 & $\ldots$ \\
\hline $073.4+01.5$ & IRAS 20084+3604 & 2059170912744153216 & 0.34 & 302.57493 & +36.21924 & 16.6 & 0.119 & 0.043 & $\ldots$ \\
\hline $073.7+00.0$ & $\begin{array}{l}\text { IPHAS } \\
\text { J201714.26+353927.4 }\end{array}$ & 2057383136875448192 & 0.96 & 304.30945 & +35.65765 & 20.4 & -0.506 & 0.670 & $\ldots$ \\
\hline $075.0-07.2$ & $\mathrm{~Pa} 27$ & 1859955657931121536 & 1.00 & 312.24320 & +32.30410 & 12.3 & 0.686 & 0.011 & $\ldots$ \\
\hline $075.3+05.5$ & $\mathrm{~Pa} 22$ & 2072773550888053888 & 1.00 & 299.55449 & +39.91134 & 20.1 & -1.381 & 0.487 & \\
\hline $075.5+01.7$ & Ju 1 & 2060926897208561280 & 1.00 & 303.83939 & +38.04550 & 19.3 & 0.876 & 0.241 & $1.65_{-0.43}^{+0.56}$ \\
\hline $075.7+35.8$ & $\mathrm{Sa} 4-1$ & 1414294114523211904 & 1.00 & 258.45980 & +49.26971 & 13.7 & 0.183 & 0.028 & $5.87_{-0.84}^{+0.17}$ \\
\hline $075.9+11.6$ & AMU 1 & 2125895669204184832 & 1.00 & 292.78700 & +43.41602 & 13.6 & 0.630 & 0.026 & $1.58_{-0.06}^{+0.07}$ \\
\hline $076.3+14.1$ & Pa 5 & 2127040806264002944 & 1.00 & 289.87708 & +44.76183 & 15.7 & 0.434 & 0.042 & $2.35_{-0.20}^{+0.23}$ \\
\hline $076.3-02.5$ & MaCCo 2 & 2056719860792120192 & 1.00 & 308.81675 & +36.19729 & 12.7 & 0.313 & 0.010 & \\
\hline $076.4+01.8$ & KjPn 3 & 2061352305127905280 & 1.00 & 304.31442 & +38.83993 & 17.5 & 0.223 & 0.076 & $12.72_{-580}^{+6.06}$ \\
\hline $076.6+05.7$ & DSH J2 & 2075178839939632128 & 0.45 & 300.17382 & +41.15800 & 20.4 & 2.244 & 0.612 & \\
\hline $076.8-08.1$ & $\mathrm{~Pa} 28$ & 1865874672618774656 & 1.00 & 314.54561 & +33.14254 & 18.8 & 0.295 & 0.163 & \\
\hline $077.5+03.7$ & KjPn 1 & 2062501642691193984 & 0.99 & 303.10009 & +40.75815 & 19.7 & -1.673 & 0.545 & $7.13_{-171}^{+1.78}$ \\
\hline $077.6+14.7$ & Abell 61 & 2127684982639844224 & 1.00 & 289.79251 & +46.24777 & 17.3 & 0.641 & 0.061 & $1.69^{+0.19}$ \\
\hline $077.7+03.1$ & $\mathrm{KjPn} 2$ & 2062521502600693376 & 0.71 & 303.84241 & +40.57921 & 21.6 & & & $\ldots$ \\
\hline $078.4-07.2$ & Pa 29 & 1866682878077985920 & 0.98 & 314.93108 & +34.90647 & 20.7 & -0.130 & 1.301 & \\
\hline $078.5+18.7$ & NGC 6742 & 2131594128728400128 & 1.00 & 284.83272 & +48.46537 & 19.6 & 0.243 & 0.290 & $4.96_{-1.44}^{+1.51}$ \\
\hline $079.1-02.1$ & StDr 27 & 2063541643232593408 & 0.96 & 310.53166 & +38.63572 & 20.5 & 1.038 & 0.618 & $\ldots$ \\
\hline $079.6+05.8$ & M 4-17 & 2081048892007659648 & 0.99 & 302.25800 & +43.72867 & 20.5 & 0.329 & 0.551 & \\
\hline $079.8+08.6$ & Kn 27 & 2079241535416131712 & 0.87 & 299.00363 & +45.38786 & 17.8 & 0.285 & 0.081 & \\
\hline $079.8-10.2$ & Alves 1 & 1866922365452368768 & 1.00 & 318.77773 & +33.97186 & 18.3 & 0.604 & 0.127 & $1.83_{-029}^{+0.36}$ \\
\hline $079.9+06.4$ & K 3-56 & 2081894618310233856 & 1.00 & 301.73070 & +44.23855 & 20.4 & 0.692 & 0.699 & \\
\hline $080.3-10.4$ & MWP 1 & 1855295171732158080 & 1.00 & 319.28448 & +34.20766 & 13.0 & 1.991 & 0.035 & $0.50_{-0.01}^{+0.01}$ \\
\hline $081.2-14.9$ & Abell 78 & 1850685091269441792 & 1.00 & 323.87241 & +31.69596 & 13.1 & 0.588 & 0.028 & $1.66_{-0.07}^{+0.08}$ \\
\hline $082.1+07.0$ & NGC 6884 & 2082491657427348352 & 0.97 & 302.59850 & +46.46098 & 16.5 & -0.465 & 0.119 & $4.93_{-0.66}^{+0.01}$ \\
\hline $082.1-07.8$ & Kn 24 & 1868658082001121664 & 1.00 & 318.40706 & +37.26046 & 19.3 & 0.534 & 0.241 & $1.76_{-0.34}^{+0.66}$ \\
\hline $082.5+02.4$ & StDr Objet 2 & 2069825901942263936 & 0.96 & 308.37967 & +44.14585 & 16.0 & 0.764 & 0.033 & \\
\hline $082.5+11.3$ & NGC 6833 & 2086764737566091136 & 1.00 & 297.44402 & +48.96112 & 13.0 & 1.014 & 0.384 & $17.05_{-5.56}^{+5.28}$ \\
\hline $082.5-06.2$ & Kn 25 & 1872003075545516032 & 0.93 & 317.33438 & +38.60184 & 20.8 & $\ldots$ & $\ldots$ & $\ldots$ \\
\hline $083.3+02.9$ & $\begin{array}{l}\text { IPHAS } \\
\text { J203346.47+450840.1 }\end{array}$ & 2070731860869105920 & 1.00 & 308.44366 & +45.14443 & 20.9 & $\ldots$ & $\ldots$ & 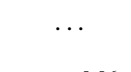 \\
\hline $083.5+12.7$ & NGC 6826 & 2135352396915239808 & 1.00 & 296.20056 & +50.52502 & 10.6 & 0.771 & 0.036 & $1.29_{-0.06}^{+0.06}$ \\
\hline $083.9-08.4$ & K 3-81 & 1964102533181902080 & 1.00 & 320.56404 & +38.12088 & 15.7 & 0.170 & 0.039 & \\
\hline $084.2-04.2$ & K 3-80 & 1969244880344005888 & 1.00 & 316.91528 & +40.96454 & 17.7 & 0.150 & 0.080 & $8.82^{+3.04}$ \\
\hline $084.6-07.9$ & Kn 26 & 1965268354805776896 & 1.00 & 320.78888 & +38.97003 & 17.1 & 0.445 & 0.063 & \\
\hline $084.9+04.4$ & Abell 71 & 2071605220993676544 & 0.98 & 308.09665 & +47.34726 & 19.2 & 1.027 & 0.208 & $1.11_{-0.19}^{+0.25}$ \\
\hline $084.9-03.4$ & NGC 7027 & 1969656406922355072 & 1.00 & 316.75735 & +42.23603 & 15.8 & 2.166 & 0.341 & $0.53_{-0.09}^{+0.12}$ \\
\hline $085.3+52.3$ & Jacoby 1 & 1595941441250636672 & 1.00 & 230.44399 & +52.36779 & 15.6 & 1.295 & 0.041 & $0.78_{-0.02}^{+0.03}$ \\
\hline $086.1+05.4$ & We $1-10$ & 2179832585761932032 & 1.00 & 307.96817 & +48.88048 & 18.0 & 0.490 & 0.082 & $2.07_{-0.31}^{+0.31}$ \\
\hline $086.5-08.8$ & Hu 1-2 & 1965932567203447296 & 0.97 & 323.28461 & +39.63598 & 17.4 & -0.614 & 0.220 & $6.07_{-118}^{+1.26}$ \\
\hline $086.9-03.4$ & Ou 5 & 1970016153397634048 & 1.00 & 318.58346 & +43.69333 & 18.6 & -0.013 & 0.109 & $7.35_{-1.50}^{+1.61}$ \\
\hline $088.7+04.6$ & K 3-78 & 2168422854828955776 & 1.00 & 311.34454 & +50.37773 & 20.4 & 1.740 & 0.823 & $14.24_{-4.22}^{+4.15}$ \\
\hline $088.7-01.6$ & NGC 7048 & 2164192930525717248 & 1.00 & 318.56328 & +46.28807 & 19.0 & 0.627 & 0.212 & $1.78_{-0.37}^{+0.45}$ \\
\hline $089.0+00.3$ & NGC 7026 & 2165238733564304768 & 1.00 & 316.57737 & +47.85187 & 15.5 & 0.313 & 0.030 & $2.74_{-0.18}^{-0.37}$ \\
\hline $089.1+08.7$ & Pa 39 & 2181841672689968128 & 1.00 & 306.39402 & +53.21242 & 14.7 & 0.161 & 0.020 & -0 \\
\hline $089.3+12.8$ & $\mathrm{~Pa} 4$ & 2233941236591666688 & 0.41 & 300.61788 & +55.55523 & 19.1 & 0.457 & 0.190 & \\
\hline $089.3-02.2$ & M 1-77 & 1971995510535755648 & 1.00 & 319.78069 & +46.31309 & 11.9 & 0.344 & 0.010 & $2.65_{-007}^{+0.07}$ \\
\hline $089.8-00.6$ & Sh $1-89$ & 2164519416761365248 & 1.00 & 318.53146 & +47.77253 & 20.0 & 0.532 & 0.403 & $1.89_{-0.46}^{+0.56}$ \\
\hline $089.8-05.1$ & IC 5117 & 1971049178931721600 & 0.20 & 323.12881 & +44.59699 & 18.5 & & & \\
\hline $090.8+06.1$ & $\mathrm{~Pa} 3$ & 2182418607053195776 & 1.00 & 311.54434 & +52.95147 & 15.9 & 0.986 & 0.028 & $\cdots$ \\
\hline $091.0+20.0$ & WPS 28 & 2239709863001566848 & 0.99 & 289.59958 & +59.99820 & 14.8 & 9.440 & 0.033 & \\
\hline $091.0-06.3$ & Sh2-123 & 1973817302524302080 & 1.00 & 325.56460 & +44.46788 & 18.1 & 1.504 & 0.108 & \\
\hline $091.6-04.8$ & K 3-84 & 1977145043182913408 & 1.00 & 324.70416 & +46.00768 & 19.4 & -0.977 & 0.376 & $9.38_{-228}^{+2.37}$ \\
\hline $093.3-00.9$ & K 3-82 & 2170991623233531264 & 0.87 & 322.71446 & +50.00233 & 19.2 & -0.119 & 0.208 & $3.96_{-0.85}^{+0.93}$ \\
\hline $093.3-02.4$ & M 1-79 & 1978860762361597312 & 1.00 & 324.25540 & +48.93445 & 19.1 & 0.602 & 0.187 & $2.21_{-0.56}^{+0.72}$ \\
\hline $093.4+05.4$ & NGC 7008 & 2188752549939971200 & 1.00 & 315.13687 & +54.54317 & 13.7 & 1.554 & 0.075 & $0.64_{-0.03}^{+0.03}$ \\
\hline $093.4-03.2$ & StDr 2 & 1978028088466446848 & 1.00 & 325.09209 & +48.36450 & 17.9 & 1.552 & 0.089 & $\ldots$ \\
\hline
\end{tabular}


Table A.1: continued.

\begin{tabular}{|c|c|c|c|c|c|c|c|c|c|}
\hline PN G & PN name & Gaia source ID & reliability & $\begin{array}{c}\text { RA } \\
(\text { deg })\end{array}$ & $\begin{array}{c}\text { Dec } \\
(\mathrm{deg})\end{array}$ & $\begin{array}{c}\mathrm{G} \\
(\mathrm{mag})\end{array}$ & $\begin{array}{c}\omega \\
(\mathrm{mas})\end{array}$ & $\begin{array}{c}\sigma_{\omega} \\
(\mathrm{mas})\end{array}$ & $\begin{array}{c}\text { distance } \\
(\mathrm{kpc})\end{array}$ \\
\hline $093.9-00.1$ & IRAS $21282+5050$ & 2171652769005709568 & 0.70 & 322.49360 & +51.06675 & 13.8 & 0.268 & 0.019 & $3.40^{+0.23}$ \\
\hline $094.0+27.4$ & K 1-16 & 2160562927224840576 & 1.00 & 275.46708 & +64.36482 & 15.0 & 0.589 & 0.035 & $1.75_{-0.10}^{+0.12}$ \\
\hline $094.5-00.8 \mathrm{a}$ & LDu 1 & 2171509218320989440 & 1.00 & 324.02398 & +50.90258 & 21.1 & & & \\
\hline $095.2+00.7$ & K 3-62 & 2172595604536445184 & 1.00 & 322.95910 & +52.56432 & 18.7 & 0.257 & 0.242 & $6.90_{-229}^{+2.40}$ \\
\hline $095.2+07.8$ & Abell 73 & 2190074983250182400 & 0.98 & 314.11231 & +57.43414 & 20.7 & 0.640 & 1.638 & $2.53_{-073}^{+0.94}$ \\
\hline $095.2+25.4$ & Kn 59 & 2256870662532650624 & 1.00 & 280.42456 & +65.19950 & 20.9 & & & \\
\hline $095.7-00.7$ & $\mathrm{~Pa} \mathbf{J} 2141.2+5146$ & 2173012693091458304 & 0.95 & 325.31122 & +51.76638 & 17.8 & 0.332 & 0.087 & \\
\hline $096.3+02.3$ & K 3-61 & 2177492137825896576 & 0.97 & 322.50313 & +54.45755 & 18.1 & 0.108 & 0.110 & $7.83_{-1.99}^{+2.16}$ \\
\hline $096.4+29.9$ & NGC 6543 & 1633325248915154176 & 1.00 & 269.63918 & +66.63296 & 11.2 & 0.732 & 0.029 & $1.31_{-0.05}^{+0.05}$ \\
\hline $097.4+12.3$ & $\mathrm{KnFe} 1$ & 2195278765626033024 & 1.00 & 309.53828 & +61.91747 & 20.4 & -0.784 & 0.737 & \\
\hline $097.6-02.4$ & M 2-50 & 1981056379700822144 & 0.97 & 329.42409 & +51.69424 & 18.4 & 0.001 & 0.146 & $6.95_{-159}^{+1.70}$ \\
\hline $098.1+02.4$ & K 3-63 & 2174674334350252416 & 0.84 & 324.79956 & +55.76784 & 18.6 & 0.062 & 0.168 & $8.51_{-2.47}^{+2.65}$ \\
\hline $098.1-28.5$ & Fr 2-18 & 1885477727979289088 & 1.00 & 347.86713 & +29.49273 & 15.4 & 3.947 & 0.045 & $\ldots$ \\
\hline $098.2+04.9$ & K 3-60 & 2178857907371556864 & 0.79 & 321.86068 & +57.65149 & 19.1 & & & \\
\hline $098.3-04.9$ & $\mathrm{~Pa} 41$ & 1976783887984709120 & 1.00 & 332.55684 & +50.07593 & 17.4 & 0.469 & 0.072 & \\
\hline $099.1+05.7$ & KTC 1 & 2179544655458448512 & 0.91 & 322.04572 & +58.87639 & 17.4 & 0.196 & 0.061 & $6.22_{-1.53}^{+1.97}$ \\
\hline $099.7-08.8$ & HaWe 15 & 1986574557983855104 & 1.00 & 337.63930 & +47.52314 & 18.3 & 0.830 & 0.122 & $1.35_{-0.18}^{+0.23}$ \\
\hline $100.0-08.7$ & Me 2-2 & 1986956260321582720 & 1.00 & 337.93198 & +47.80107 & 14.1 & & & \\
\hline $100.3+02.8$ & Cr 1 & 2202260634408052224 & 1.00 & 327.29870 & +57.45547 & 17.2 & 0.733 & 0.063 & $1.33_{-0.10}^{+0.12}$ \\
\hline $100.4+04.6$ & PM 1-333 & 2179381824658713856 & 1.00 & 325.24607 & +58.97845 & 18.9 & 0.415 & 0.176 & $2.66_{-0.60}^{+0.70}$ \\
\hline $100.6-05.4$ & IC 5217 & 2000514441288746752 & 0.99 & 335.98212 & +50.96678 & 15.6 & 0.189 & 0.073 & $4.83_{-0.91}^{+1.06}$ \\
\hline $101.4-01.9$ & StDr 43 & 2005036900103208704 & 1.00 & 334.07596 & +54.31167 & 18.9 & 0.769 & 0.177 & \\
\hline $101.8+04.3$ & Pa 57 & 2202763076866529024 & 0.63 & 327.75694 & +59.59041 & 16.5 & 0.131 & 0.047 & \\
\hline $101.8+08.7$ & NGC 7076 & 2216998964996270208 & 0.98 & 321.59815 & +62.89241 & 17.1 & 0.294 & 0.052 & $3.07_{-0.36}^{+0.43}$ \\
\hline $101.9+17.0$ & ETHOS 4 & 2250116087725032192 & 0.77 & 305.90560 & +68.12069 & 18.8 & & & \\
\hline $102.5+02.6$ & 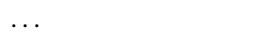 & 2199751926819942144 & 0.71 & 330.77463 & +58.65315 & 20.6 & -0.473 & 0.912 & $\ldots$ \\
\hline $102.7+03.7$ & $\begin{array}{l}\text { IPHAS } \\
\text { J215919.15+594009.9 }\end{array}$ & 2202908173733325312 & 0.98 & 329.82979 & +59.66938 & 20.1 & 0.058 & 0.419 & s. \\
\hline $102.8-05.0$ & Abell 80 & 2002155050070646912 & 1.00 & 338.69003 & +52.43494 & 19.6 & 0.621 & 0.287 & $2.12_{-0.53}^{+0.60}$ \\
\hline $102.9-02.3$ & Abell 79 & 2004936573978252672 & 0.99 & 336.57192 & +54.82728 & 16.6 & 0.224 & 0.047 & $3.67_{-0.43}^{+0.53}$ \\
\hline $103.2+00.6$ & M 2-51 & 2198557685406698368 & 0.76 & 334.01667 & +57.47584 & 19.8 & 0.283 & 0.367 & $1.95_{-0.43}^{+0.43}$ \\
\hline $103.7+07.2$ & Kn 30 & 2216463232261024896 & 0.98 & 326.85124 & +63.08598 & 20.1 & -0.165 & 0.502 & $\ldots$ \\
\hline $103.9+05.5$ & Dr 5 & 2204179277897545856 & 1.00 & 329.39798 & +61.85803 & 20.4 & -0.176 & 0.498 & \\
\hline $103.9-03.7$ & StDr 96 & 2003108635889408384 & 0.40 & 339.25172 & +54.04738 & 20.8 & -0.771 & 1.463 & $\ldots$ \\
\hline $104.1+01.0$ & B1 2-1 & 2200102945912268800 & 0.81 & 335.06939 & +58.23786 & 19.5 & & & \\
\hline $104.1+07.9$ & NGC 7139 & 2216734669893916800 & 1.00 & 326.53564 & +63.79140 & 18.7 & 0.117 & 0.301 & $2.23_{-0.45}^{+0.49}$ \\
\hline $104.2-29.6$ & Jn 1 & 2871119705335735552 & 1.00 & 353.97219 & +30.46843 & 16.0 & 1.011 & 0.065 & $0.99_{-0.06}^{+0.07}$ \\
\hline $104.4-01.6$ & M 2-53 & 2006751661516849536 & 0.98 & 338.07381 & +56.17383 & 20.8 & & & $\ldots$ \\
\hline $105.4-14.0$ & Kn 130 & 1941078175572093696 & 1.00 & 348.27200 & +45.43838 & 16.5 & 0.497 & 0.054 & \\
\hline $105.9-20.0$ & Fr $1-6$ & 1923253820774422272 & 1.00 & 351.81644 & +40.02323 & 14.8 & 0.702 & 0.034 & \\
\hline $106.5-17.6$ & NGC 7662 & 1924818288379268736 & 1.00 & 351.47430 & +42.53494 & 13.9 & 0.569 & 0.033 & $1.65_{-0.08}^{+0.09}$ \\
\hline $107.0+21.3$ & K 1-6 & 2288467186442571008 & 1.00 & 301.05949 & +74.42665 & 12.3 & 3.864 & 0.030 & $0.26_{-0.00}^{+0.00}$ \\
\hline $107.2+14.9$ & Dr 21 & 1938255660503776000 & 1.00 & 350.95788 & +45.28171 & 17.5 & 0.648 & 0.095 & \\
\hline $107.4-02.6$ & K 3-87 & 2004012155267889792 & 0.98 & 343.77888 & +56.70861 & 19.9 & 0.071 & 0.372 & $11.56_{-351}^{+3.55}$ \\
\hline $107.6-13.3$ & Vy $2-3$ & 1941633699525901440 & 1.00 & 350.74125 & +46.89946 & 14.6 & 0.161 & 0.031 & $5.98_{-0.94}^{+1.27}$ \\
\hline $107.7+07.8$ & IsWe 2 & 2218688261534278912 & 1.00 & 333.34383 & +65.89871 & 18.1 & 1.221 & 0.095 & $0.77_{-0.05}^{+0.05}$ \\
\hline $107.8+02.3$ & NGC 7354 & 2201080755349789568 & 1.00 & 340.08277 & +61.28574 & 17.3 & 0.476 & 0.069 & $1.81_{-0.17}^{+0.19}$ \\
\hline $109.4+07.7$ & Kn 31 & 2218796151111558016 & 1.00 & 336.91327 & +66.73599 & 20.1 & 0.590 & 0.409 & $3.80_{-1.11}^{+1.13}$ \\
\hline $110.1+01.9$ & PM 1-3 & 2206963279991284224 & 0.50 & 344.71540 & +61.96186 & 21.4 & & & $\ldots$ \\
\hline $110.2+03.3$ & $\begin{array}{l}\text { IPHASX } \\
\text { J225453.2+631827 }\end{array}$ & 2208061000617183872 & 0.30 & 343.72194 & +63.30734 & 20.2 & 1.418 & 0.472 & $\ldots$ \\
\hline $110.6-12.9$ & K 1-20 & 4 & 1.00 & 12 & +48.20 & 20.6 & -1.008 & 1.106 & \\
\hline $111.2+07.0$ & KjPn 6 & 2212671428967795840 & 0.96 & 342.25906 & +67.02729 & 16.8 & 0.082 & 0.053 & $\ldots$ \\
\hline $111.8-02.8$ & $\mathrm{Hb} 12$ & 2009637806507452672 & 1.00 & 351.56177 & +58.18182 & 11.8 & & & \\
\hline $112.9-10.2$ & Abell 84 & 1944244047502655232 & 1.00 & 356.93355 & +51.39920 & 18.5 & 0.418 & 0.142 & $2.22_{-0.37}^{+0.43}$ \\
\hline $113.3+06.4$ & Kn 32 & 2212362019520749312 & 0.71 & 347.43169 & +67.39398 & 20.3 & & 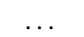 & \\
\hline $114.0-04.6$ & Abell 82 & 1998212476247082880 & 0.58 & 356.44893 & +57.06622 & 14.4 & 0.522 & 0.017 & $1.81_{-0.05}^{+0.06}$ \\
\hline $114.4+00.0$ & $\begin{array}{l}\text { IPHASX } \\
\text { J233841.2+614146 }\end{array}$ & 2012536875076571776 & 0.92 & 354.67157 & +61.69606 & 20.0 & 0.252 & 0.359 & \\
\hline $114.7-01.2$ & $\begin{array}{l}\text { IPHASX } \\
\text { J234403.8+603242 }\end{array}$ & 2011797217286839168 & 0.23 & 356.01663 & +60.54490 & 15.4 & 0.592 & 0.026 & \\
\hline $116.0-04.8$ & $\begin{array}{l}\text { IPHASX } \\
\text { J000021.4+572207 }\end{array}$ & 422587471554771968 & 1.00 & 0.08912 & +57.36880 & 16.7 & 26 & 0.050 & \\
\hline $116.2+08.5$ & M 2-55 & 2215132685747021056 & 0.98 & 352.96454 & +70.37042 & 19.7 & -0.425 & 0.291 & $3.01_{-0.57}^{+0.61}$ \\
\hline $117.5+05.3$ & Dr 27 & 422428519103328128 & 1.00 & 2.76432 & +57.17670 & 17.7 & 1.008 & 0.091 & \\
\hline $117.5+18.9$ & IC 1454 & 2286089355109979008 & 1.00 & 340.60251 & +80.44201 & 18.8 & -0.131 & 0.170 & $5.75_{-1.18}^{+1.27}$ \\
\hline $117.9+01.6$ & IRAS $00032+6348$ & 431675381825745408 & 0.99 & 1.47282 & +64.08769 & 19.2 & 0.627 & 0.239 & \\
\hline $118.0-08.6$ & PN VV 2 & 419326659360627968 & 1.00 & 4.67573 & +53.87216 & 14.1 & 0.196 & 0.031 & \\
\hline $118.8-74.7$ & NGC 246 & 2376592910265354368 & 1.00 & 11.76385 & -11.87198 & 11.8 & 1.799 & 0.079 & $0.54_{-0.02}^{+0.02}$ \\
\hline $119.1+12.4$ & Kn 50 & 2229266628545001984 & 1.00 & 358.54726 & +74.92611 & 19.3 & 0.083 & 0.245 & $2.89_{-0.58}^{+0.63}$ \\
\hline
\end{tabular}


Table A.1: continued.

\begin{tabular}{|c|c|c|c|c|c|c|c|c|c|}
\hline PN G & PN name & Gaia source ID & reliability & $\begin{array}{c}\text { RA } \\
(\text { deg })\end{array}$ & $\begin{array}{c}\text { Dec } \\
(\mathrm{deg})\end{array}$ & $\begin{array}{c}\mathrm{G} \\
(\mathrm{mag})\end{array}$ & $\begin{array}{c}\omega \\
(\mathrm{mas})\end{array}$ & $\begin{array}{c}\sigma_{\omega} \\
(\mathrm{mas})\end{array}$ & $\begin{array}{c}\text { distance } \\
(\mathrm{kpc})\end{array}$ \\
\hline $119.6-06.1$ & Hu 1-1 & 421287436486020608 & 0.99 & 7.06489 & +55.96518 & 19.3 & 1.522 & 0.541 & $5.31^{+1.84}$ \\
\hline $120.0+09.8$ & NGC 40 & 537481007814722688 & 1.00 & 3.25413 & +72.52195 & 11.5 & 0.561 & 0.017 & $1.62_{-0.04}^{+0.05}$ \\
\hline $120.2-05.3$ & Sh 2-176 & 421836329014389248 & 0.38 & 7.97199 & +57.38022 & 18.5 & 0.901 & 0.145 & $0.97_{-0.09}^{+0.11}$ \\
\hline $120.4-01.3$ & Ou 2 & 430204780732841600 & 1.00 & 7.73643 & +61.40952 & 19.3 & 0.768 & 0.218 & $\ldots$ \\
\hline $121.7+11.4$ & Hu 2 & 538591274040363520 & 1.00 & 8.48961 & +74.31093 & 20.2 & 0.437 & 0.504 & \\
\hline $122.1-04.9$ & Abell 2 & 425100439735384192 & 1.00 & 11.39479 & +57.95980 & 19.9 & 0.637 & 0.422 & $3.99_{-1.21}^{+1.22}$ \\
\hline $123.0+04.6$ & $\mathrm{~Pa} 30$ & 526287189172936320 & 1.00 & 13.29666 & +67.50066 & 15.4 & 0.407 & 0.026 & $2.33_{-0.13}^{-0.215}$ \\
\hline $123.6+09.9$ & $\mathrm{~Pa} 154$ & 535212951548319616 & 0.98 & 15.35452 & +72.76480 & 20.9 & & & \\
\hline $123.6+34.5$ & IC 3568 & 1720138697015345024 & 1.00 & 188.27862 & +82.56393 & 12.9 & 0.441 & 0.037 & $2.20_{-0.16}^{+0.19}$ \\
\hline $124.0+02.9$ & KLSS 2-7 & 525857348844254208 & 0.99 & 15.60190 & +65.77569 & 21.2 & & & \\
\hline $124.3-07.7$ & WeSb 1 & 423384961080344960 & 1.00 & 15.22543 & +55.06667 & 14.8 & 0.271 & 0.024 & $3.21_{-0.21}^{+0.24}$ \\
\hline $125.4+08.9$ & Dr 36 & 411078093067339136 & 1.00 & 16.91690 & +53.87332 & 18.8 & 0.851 & 0.196 & \\
\hline $126.3+02.9$ & K 3-90 & 525275913347840512 & 1.00 & 21.24421 & +65.64336 & 18.8 & 0.405 & 0.168 & $5.92_{-2.23}^{+2.42}$ \\
\hline $126.6+01.3$ & $\begin{array}{l}\text { IPHASX } \\
\text { J012507.9+635652 }\end{array}$ & 512781539949346816 & 1.00 & 21.28314 & +63.94799 & 18.9 & 0.326 & 0.198 & $\ldots$ \\
\hline $126.7-15.4$ & Fr 2-30 & 401414034397108352 & 0.23 & 18.32132 & +47.23320 & 19.4 & 1.198 & 0.267 & $\ldots$ \\
\hline $127.6-01.1$ & $\begin{array}{l}\text { IPHASX } \\
\text { J013108.9+612258 }\end{array}$ & 510742396851462400 & 1.00 & 22.78751 & +61.38302 & 20.5 & 0.177 & 0.640 & \\
\hline $127.6-13.5$ & PaStDr 3 & 400211958948711552 & 1.00 & 19.87035 & +49.01925 & 13.4 & 1.240 & 0.033 & \\
\hline $128.0-04.1$ & Sh 2-188 & 509206447837376128 & 1.00 & 22.63829 & +58.41396 & 17.4 & 1.060 & 0.073 & $0.85_{-0.04}^{+0.05}$ \\
\hline $129.2-02.0$ & We $2-5$ & 509636112062310016 & 1.00 & 25.65783 & +60.16310 & 20.3 & 0.221 & 0.568 & $2.09_{-0.51}^{+0.54}$ \\
\hline $129.6+03.4$ & $\begin{array}{l}\text { IPHASX } \\
\text { J015624.9+652830 }\end{array}$ & 518353624360945152 & 0.99 & 29.10449 & +65.47513 & 18.8 & 0.761 & 0.176 & $\ldots$ \\
\hline $129.6-05.6$ & KLSS 2-8 & 409721566300550656 & 1.00 & 25.02435 & +56.58183 & 19.8 & 1.127 & 0.318 & $3.06_{-1.57}^{+1.63}$ \\
\hline $130.2+01.3$ & IC 1747 & 511904404556131200 & 1.00 & 29.39870 & +63.32180 & 15.8 & 0.262 & 0.038 & $3.48_{-0.38}^{+0.45}$ \\
\hline $130.3-11.7$ & M 1-1 & 405830570741274752 & 0.96 & 24.33067 & +50.46982 & 17.7 & 0.144 & 0.125 & $8.32_{-2.32}^{+2.58}$ \\
\hline $130.9-10.5$ & NGC 650/1 & 406328443354164480 & 0.90 & 25.58192 & +51.57541 & 17.4 & 0.294 & 0.203 & $1.36_{-0.17}^{+0.19}$ \\
\hline $131.0+03.7$ & $\mathrm{Fe} 8$ & 515511318086256768 & 1.00 & 32.54356 & +65.42064 & 19.3 & 0.511 & 0.219 & \\
\hline $131.4-05.4$ & BV 5-3 & 504819018483991168 & 1.00 & 28.26254 & +56.40537 & 20.5 & 0.264 & 0.724 & $5.44_{-1.55}^{+1.57}$ \\
\hline $131.5+02.6$ & Abell 3 & 515007024509005440 & 0.30 & 33.02775 & +64.15065 & 17.2 & 0.436 & 0.066 & $2.27_{-0.28}^{+0.36}$ \\
\hline $132.8+02.0$ & $\begin{array}{l}\text { IPHASX } \\
\text { J022045.0+631134 }\end{array}$ & 513965507818493312 & 0.97 & 35.18811 & +63.19284 & 20.2 & 0.759 & 0.691 & \\
\hline $134.3-43.2$ & Pre 8 & 2786087079519147520 & 1.00 & 21.65025 & +18.85510 & 18.1 & 0.215 & 0.152 & \\
\hline $135.6+01.0$ & WeBo 1 & 465640807845756160 & 1.00 & 40.05989 & +61.15465 & 13.8 & 0.635 & 0.023 & $1.49_{-0.05}^{+0.05}$ \\
\hline $135.9+55.9$ & TS 1 & 846615127231002880 & 1.00 & 178.35307 & +59.66584 & 18.0 & 0.026 & 0.117 & \\
\hline $136.1+04.9$ & Abell 6 & 467936205865972352 & 1.00 & 44.67447 & +64.50172 & 18.4 & 0.869 & 0.143 & $1.20_{-017}^{+0.21}$ \\
\hline $136.3+05.5$ & HFG 1 & 468033345145186816 & 1.00 & 45.94596 & +64.90984 & 14.0 & 1.413 & 0.015 & $0.69_{-0.01}^{+0.81}$ \\
\hline $136.6+61.9$ & PN G136.7+61.9 & 1573011744729727104 & 1.00 & 181.86839 & +54.02471 & 15.9 & 0.873 & 0.055 & $1.17_{-0.07}^{+0.88}$ \\
\hline $136.8-13.2$ & Kn 58 & 354941216942517504 & 1.00 & 33.11600 & +47.45280 & 19.5 & 0.153 & 0.344 & $5.76_{-153}^{+1.57}$ \\
\hline $137.6-30.0$ & Fr 2-22 & 304311150320629376 & 1.00 & 27.72617 & +31.12964 & 14.3 & 1.371 & 0.037 & \\
\hline $138.1+04.1$ & Sh 2-200 & 466746538582576640 & 1.00 & 47.74525 & +62.79858 & 16.2 & 1.106 & 0.286 & $0.88_{-014}^{+0.17}$ \\
\hline $138.8+02.8$ & IC 289 & 463228376251556224 & 1.00 & 47.58040 & +61.31690 & 16.4 & 0.634 & 0.049 & $1.54_{-0.11}^{+0.14}$ \\
\hline $139.0+03.2$ & $\begin{array}{l}\text { IPHASX } \\
\text { J031345.5+613707 }\end{array}$ & 463616778732232064 & 1.00 & 48.43800 & +61.61833 & 20.6 & 2.493 & 0.920 & \\
\hline $139.3+04.8$ & KK 26 & 463781877277363712 & 0.71 & 50.77212 & +62.78515 & 16.3 & 0.562 & 0.062 & \\
\hline $141.7-07.8$ & Abell 5 & 440314343470162304 & 0.98 & 43.06289 & +50.59830 & 19.7 & 0.581 & 0.385 & $2.98_{-0.95}^{+1.02}$ \\
\hline $142.1+03.4$ & K 3-94 & 450339621774948736 & 0.98 & 54.03368 & +60.06273 & 20.9 & & & -0.95 \\
\hline $143.5+38.7$ & WPS 46 & 1071088328781771392 & 1.00 & 141.96402 & +69.23905 & 10.7 & 1.030 & 0.017 & \\
\hline $144.1+06.1$ & NGC 1501 & 473712872456844544 & 1.00 & 61.74749 & +60.92061 & 14.2 & 0.579 & 0.019 & $1.63_{-0.05}^{+0.05}$ \\
\hline $144.3-15.5$ & Abell 4 & 337112571475961344 & 1.00 & 41.34855 & +42.55127 & 19.7 & -0.078 & 0.519 & $7.76_{-2.28}^{+2.33}$ \\
\hline $144.8+65.8$ & LTNF 1 & 786919754746647424 & 1.00 & 179.43686 & +48.93836 & 15.1 & 0.708 & 0.026 & $1.36_{-0.05}^{+0.09}$ \\
\hline $145.4+05.7$ & $\operatorname{Dr} 26$ & 470580325172742912 & 1.00 & 61.70656 & +59.72939 & 19.0 & 0.900 & 0.246 & -0.05 \\
\hline $146.1-08.1$ & Fr 2-23 & 435527383380748416 & 1.00 & 48.69130 & +48.20162 & 14.3 & 4.320 & 0.039 & \\
\hline $146.7+07.6$ & M 4-18 & 471908436438311680 & 1.00 & 66.46182 & +60.12021 & 13.7 & 0.153 & 0.016 & $5.52_{-0.46}^{+0.55}$ \\
\hline $147.1-09.0$ & HaWe 3 & 434853485833190528 & 1.00 & 49.14183 & +46.89369 & 17.9 & 0.106 & 0.111 & $7.62_{-1.85}^{+0.46}$ \\
\hline $147.2+08.3$ & Kn 33 & 471551198232719744 & 0.74 & 68.15899 & +60.33732 & 19.6 & 1.008 & 0.323 & \\
\hline $147.4-02.3$ & M 1-4 & 443728125217394688 & 1.00 & 55.43092 & +52.28340 & 18.1 & 0.161 & 0.202 & $5.29_{-139}^{+1.48}$ \\
\hline $147.8+04.1$ & M 2-2 & 469056092819323392 & 0.99 & 63.31262 & +56.94957 & 15.8 & 0.211 & 0.053 & $4.68_{-0.84}^{+1.08}$ \\
\hline $148.4+57.0$ & NGC 3587 & 843950873117830528 & 1.00 & 168.69880 & +55.01903 & 15.7 & 1.235 & 0.046 & $0.81_{-0.03}^{+0.03}$ \\
\hline $149.0+04.4$ & K 4-47 & 276843494433439872 & 0.98 & 65.18842 & +56.30337 & 21.8 & & $\ldots$ & \\
\hline $149.4-09.2$ & HaWe 4 & 241918950690107264 & 1.00 & 51.81422 & +45.40569 & 17.1 & 1.092 & 0.071 & $0.92_{-0.06}^{+0.06}$ \\
\hline $149.7-03.3$ & IsWe 1 & 250358801943821952 & 1.00 & 57.27473 & +50.00410 & 16.5 & 2.350 & 0.057 & $0.43_{-0.01}^{+0.061}$ \\
\hline $150.0-00.3$ & Te 5 & 251042010983671936 & 1.00 & 60.87305 & +52.14056 & 21.0 & & & $\ldots$ \\
\hline $150.1-04.5$ & $\begin{array}{l}\text { IPHASX } \\
\text { J034659.8+484900 }\end{array}$ & 248726366476289280 & 0.45 & 56.74863 & +48.81681 & 16.6 & 0.449 & 0.061 & \\
\hline $150.9-10.1$ & Bode 1 & 238540495056450048 & 0.99 & 52.80000 & +43.90422 & 12.6 & 2.306 & 0.041 & $\ldots$ \\
\hline $151.0-00.4$ & Ou 1 & 250719647917343232 & 1.00 & 61.83989 & +51.40621 & 19.3 & 0.697 & 0.294 & \\
\hline $153.7+22.8$ & Abell 16 & 1004051581576523008 & 1.00 & 100.98093 & +61.79018 & 18.6 & 0.762 & 0.173 & $1.82_{-0.44}^{+0.62}$ \\
\hline $155.9+07.7$ & $\mathrm{~Pa} 153$ & 266157955103568256 & 1.00 & 77.28176 & +53.17450 & 17.9 & 0.436 & 0.122 & $\cdots$ \\
\hline
\end{tabular}


Table A.1: continued.

\begin{tabular}{|c|c|c|c|c|c|c|c|c|c|}
\hline$\overline{\mathrm{PN} \mathrm{G}}$ & PN name & Gaia source ID & reliability & $\begin{array}{c}\text { RA } \\
(\mathrm{deg})\end{array}$ & $\begin{array}{l}\text { Dec } \\
(\mathrm{deg})\end{array}$ & $\begin{array}{c}\mathrm{G} \\
(\mathrm{mag})\end{array}$ & $\begin{array}{c}\omega \\
(\mathrm{mas})\end{array}$ & $\begin{array}{c}\sigma_{\omega} \\
(\mathrm{mas})\end{array}$ & $\begin{array}{l}\text { distance } \\
(\mathrm{kpc})\end{array}$ \\
\hline $156.3+12.5$ & HaWe 6 & 268129413812162816 & 1.00 & 84.48446 & +55.53763 & 16.5 & 3.413 & 0.066 & $T=\frac{1}{2}$ \\
\hline $157.1+04.4$ & $\begin{array}{l}\text { IPHASX } \\
\text { J045627.6+501720 }\end{array}$ & 259560266863352576 & 0.34 & 74.11753 & +50.28823 & 19.6 & 1.948 & 0.356 & $\ldots$ \\
\hline $157.3+10.3$ & $\mathrm{Hu} 4$ & 263363241360588288 & 0.28 & 82.08892 & +53.52180 & 18.6 & 3.931 & 0.723 & $\ldots$ \\
\hline $158.3-05.6 \mathrm{a}$ & Sh $2-215$ & 229213505415604864 & 0.99 & 64.36590 & +42.60117 & 18.9 & 0.112 & 0.252 & \\
\hline $158.6+00.7$ & Sh 2-216 & 254092090595748096 & 1.00 & 70.83874 & +46.70156 & 12.6 & 7.840 & 0.048 & $0.13_{-0.00}^{+0.00}$ \\
\hline $158.8+37.1$ & Abell 28 & 1040417211407001984 & 1.00 & 130.39817 & +58.23007 & 16.5 & 2.606 & 0.071 & $0.39_{-0.01}^{+0.00}$ \\
\hline $158.9+17.8$ & PuWe 1 & 997854527884948992 & 1.00 & 94.89142 & +55.61210 & 15.5 & 2.535 & 0.059 & $0.39^{+0.01}$ \\
\hline $159.0-15.1$ & IC 351 & 219833434282576000 & 1.00 & 56.88742 & +35.04684 & 16.4 & 0.305 & 0.094 & $4.69_{-1.25}^{+1.55}$ \\
\hline $160.5-00.5$ & We $1-2$ & 204885577998383872 & 1.00 & 71.67934 & +44.46706 & 20.8 & & & \\
\hline $161.2-14.8$ & IC 2003 & 218544737933650944 & 1.00 & 59.09162 & +33.87520 & 16.1 & 0.049 & 0.070 & $5.57_{-0.86}^{+0.96}$ \\
\hline $161.9-44.0$ & Fr 2-2 & 24913597751278976 & 1.00 & 40.19729 & +10.35067 & 9.2 & 3.253 & 0.105 & \\
\hline $162.1+47.9$ & WPS 54 & 1020641700210987520 & 1.00 & 147.85829 & +53.15853 & 15.3 & 3.177 & 0.045 & $\ldots$ \\
\hline $162.9-01.6$ & Weinberger 92 & 203426487411436160 & 1.00 & 72.57369 & +41.91474 & 19.7 & 1.194 & 0.573 & $\ldots$ \\
\hline $163.1-00.8$ & We $1-3$ & 204926805386682752 & 1.00 & 73.62916 & +42.27801 & 21.0 & & & \\
\hline $164.8+31.1$ & JnEr 1 & 936605992140011392 & 1.00 & 119.46507 & +53.42137 & 17.1 & 1.065 & 0.079 & $0.97_{-0.07}^{+0.08}$ \\
\hline $164.8-09.8$ & Kn 51 & 176269718435866112 & 1.00 & 66.36192 & +35.10217 & 18.2 & 1.042 & 0.184 & \\
\hline $165.4-05.2$ & Kn 37 & 175340665471792000 & 1.00 & 71.15732 & +37.65421 & 19.0 & 0.321 & 0.286 & \\
\hline $165.5-06.5$ & K 3-67 & 175182924208301824 & 0.93 & 69.94965 & +36.76194 & 17.4 & & & \\
\hline $165.5-15.2$ & NGC 1514 & 168937010969340160 & 1.00 & 62.32074 & +30.77598 & 9.3 & 2.203 & 0.017 & $0.45^{+0.00}$ \\
\hline $165.5-27.6$ & Kn 67 & 58511404626001152 & 1.00 & 53.06231 & +21.66189 & 20.3 & -1.963 & 0.807 & \\
\hline $166.1+10.4$ & IC 2149 & 196996680850087936 & 1.00 & 89.09955 & +46.10474 & 11.3 & 0.535 & 0.037 & $1.72^{+0.12}$ \\
\hline $166.6-14.7$ & Kn 132 & 165965786951040640 & 0.79 & 63.58859 & +30.39184 & 18.9 & 0.820 & 0.336 & \\
\hline $167.0-00.9$ & Abell 8 & 188439147494309632 & 1.00 & 76.66008 & +39.13572 & 20.1 & 0.703 & 0.657 & $3.40_{-108}^{+1.10}$ \\
\hline $167.4-09.1$ & K 3-66 & 172188296914590208 & 1.00 & 69.15516 & +33.65835 & 15.3 & 0.255 & 0.128 & $7.05_{-2.27}^{+2.48}$ \\
\hline $168.4+01.6$ & $\mathrm{CoMaC} 2$ & 187946497564416384 & 1.00 & 80.48764 & +39.51735 & 15.2 & 0.205 & 0.036 & $\ldots$ \\
\hline $169.4+03.8$ & Dr 32 & 191146557438088064 & 1.00 & 83.52788 & +39.93421 & 19.2 & 0.387 & 0.309 & \\
\hline $170.3+15.8$ & NGC 2242 & 965066369588651648 & 1.00 & 98.53062 & +44.77716 & 16.8 & 0.338 & 0.075 & $4.09_{-0.97}^{+1.40}$ \\
\hline $170.7+04.6$ & K 3-69 & 190211633254265472 & 1.00 & 85.34221 & +39.25222 & 17.1 & 4.237 & 0.479 & \\
\hline $171.3-25.8$ & Ba 1 & 50865027106484096 & 1.00 & 58.40166 & +19.49402 & 18.0 & 0.744 & 0.169 & $2.09^{+1.05}$ \\
\hline $173.5+03.2$ & $\mathrm{Pu} 2$ & 3455834079110379648 & 1.00 & 85.64230 & +36.15204 & 19.5 & 0.026 & 0.389 & $6.96_{-1.91}^{+1.96}$ \\
\hline $173.7-05.8$ & K 2-1 & 156455900431326976 & 1.00 & 76.78460 & +30.82179 & 18.1 & 0.448 & 0.172 & $2.04_{-037}^{+0.44}$ \\
\hline $173.7-09.2$ & & 155075188702503424 & 1.00 & 73.64034 & +28.82471 & 18.9 & -0.003 & 0.268 & \\
\hline $174.2-14.6$ & Н 3-29 & 147952487953283712 & 1.00 & 69.34764 & +25.04414 & 18.2 & 0.137 & 0.171 & $3.75^{+0.86}$ \\
\hline $174.5-03.0$ & $\begin{array}{l}\text { IPHAS } \\
\text { J052015.87+314938.8 }\end{array}$ & 180731845860251264 & 1.00 & 80.06613 & +31.82740 & 19.2 & 0.785 & 0.358 & -0.79 \\
\hline $174.6-05.2$ & $\begin{array}{l}\text { IPHASX } \\
\text { J051152.2+302751 }\end{array}$ & 156214321404677376 & 1.00 & 77.96756 & +30.46425 & 17.3 & 0.264 & 0.088 & . . \\
\hline $175.6+11.4$ & Kn 62 & 955255358615209856 & 1.00 & 95.98093 & +38.25402 & 18.6 & 0.252 & 0.191 & $\ldots$ \\
\hline $176.7-33.8$ & Alves 3 & 36321786805002880 & 1.00 & 55.80988 & +10.49393 & 16.5 & 6.887 & 0.071 & $\ldots$ \\
\hline $177.0+00.5$ & Te 2 & 3447765931507028864 & 1.00 & 85.18648 & +31.74202 & 20.9 & & & $\ldots$ \\
\hline $177.5+03.1$ & $\begin{array}{l}\text { IPHASX } \\
\text { J055226.2+323724 }\end{array}$ & 3451205783698632704 & 1.00 & 88.10914 & +32.62356 & 19.2 & 1.020 & 0.315 & . \\
\hline $178.1-04.0$ & $\begin{array}{l}\text { IPHASX } \\
\text { J052531.1+281945 }\end{array}$ & 3445383766552820352 & 0.85 & 81.37996 & +28.32918 & 19.8 & 0.847 & 0.741 & $\ldots$ \\
\hline $178.3-02.5$ & K $3-68$ & 3445837383814681472 & 1.00 & 82.89939 & +28.97812 & 19.3 & -0.598 & 0.434 & $7.75_{-1.98}^{+2.04}$ \\
\hline $181.5+00.9$ & $\mathrm{Pu} 1$ & 3431140972097482368 & 1.00 & 88.20190 & +28.09977 & 20.8 & & & $\ldots$ \\
\hline $183.0+00.0$ & $\begin{array}{l}\text { IPHASX } \\
\text { J055242.8+262116 }\end{array}$ & 3430688759283627776 & 1.00 & 88.17840 & +26.35445 & 17.1 & 0.357 & 0.087 & \\
\hline $184.0-02.1$ & M 1-5 & 3428522004121565568 & 1.00 & 86.70838 & +24.36745 & 15.9 & 0.200 & 0.086 & $5.92_{-1.54}^{+1.83}$ \\
\hline $184.6+00.6$ & K 3-70 & 3429576298334956800 & 1.00 & 89.68898 & +25.31220 & 20.8 & .. & $\ldots$ & $\ldots$ \\
\hline $185.1-03.8$ & $\begin{array}{l}\text { IPHASX } \\
\text { J054310.5+223111 }\end{array}$ & 3403487704903666048 & 1.00 & 85.79350 & +22.51951 & 20.9 & 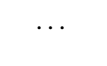 & 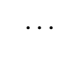 & \\
\hline $188.6+04.4$ & $\mathrm{HoCr} 1$ & 3377578125711130752 & 0.97 & 95.42076 & +23.58720 & 20.7 & & & .. $>$ > \\
\hline $189.0-00.3$ & Ra 30 & 3422942188768395264 & 1.00 & 91.13333 & +20.95022 & 20.5 & 1.784 & 1.376 & \\
\hline $189.1+19.8$ & NGC 2371-72 & 885587110718845568 & 1.00 & 111.39453 & +29.49064 & 14.8 & 0.581 & 0.046 & $1.70_{-012}^{+0.14}$ \\
\hline $189.1-07.6$ & $\mathrm{~Pa} 9$ & 3397405992554648960 & 1.00 & 84.49151 & +17.10514 & 20.4 & 0.122 & 0.799 & $5.06_{-1.09}^{+1.40}$ \\
\hline $189.8+07.7$ & M 1-7 & 3382861038566672000 & 0.99 & 99.33717 & +24.00983 & 19.8 & 1.376 & 1.217 & $6.45_{-1.198}^{+1.39}$ \\
\hline $190.3-17.7$ & $\mathrm{~J} 320$ & 3291783576991686784 & 1.00 & 76.39283 & +10.70637 & 14.5 & 0.306 & 0.058 & $3.40_{-0.59}^{+0.88}$ \\
\hline $191.0+18.3$ & Kn 68 & 872908298540811520 & 1.00 & 110.51297 & +27.22583 & 19.6 & 1.075 & 0.431 & \\
\hline $191.4+33.1$ & TK 1 & 708990321934310784 & 1.00 & 126.77302 & +31.50225 & 15.7 & 1.814 & 0.058 & $0.55_{-0.02}^{+0.02}$ \\
\hline $192.5+07.2$ & HaWe 8 & 3378885131503804544 & 1.00 & 100.04035 & +21.41713 & 18.8 & 0.829 & 0.286 & \\
\hline $192.8-24.7$ & WPS 62 & 3281864642080410112 & 1.00 & 71.76880 & +4.97804 & 16.2 & 2.271 & 0.062 & \\
\hline $193.0-04.5$ & KLSS $1-5$ & 3348734254928894208 & 1.00 & 89.28330 & +15.42532 & 20.0 & 1.051 & 0.503 & $3.88_{-1.30}^{+1.26}$ \\
\hline $193.6-09.5$ & H 3-75 & 3340384082588168960 & 0.74 & 85.18738 & +12.35625 & 13.8 & 0.249 & 0.019 & $3.47_{-0.21}^{+0.24}$ \\
\hline $194.2+02.5$ & J 900 & 3370531286968467200 & 1.00 & 96.48864 & +17.79087 & 17.1 & -0.819 & 0.289 & $4.84_{-0.07}^{+1.03}$ \\
\hline $195.4-04.0$ & $\begin{array}{l}\text { IPHASX } \\
\text { J060416.2+133250 }\end{array}$ & 3345072060869540352 & 1.00 & 91.06777 & +13.54732 & 15.0 & 0.166 & 0.030 & \\
\hline $196.6-10.9$ & NGC 2022 & 14380977664 & 1.00 & 85.52579 & +9.08627 & 15.7 & 0.438 & 0.053 & $2.20_{-0.22}^{+0.28}$ \\
\hline $197.0+05.8$ & KLSS $1-6$ & 3358740704094434176 & 1.00 & 100.85885 & +16.81428 & 18.5 & 1.439 & 0.214 & $\ldots$ \\
\hline
\end{tabular}


Table A.1: continued.

\begin{tabular}{|c|c|c|c|c|c|c|c|c|c|}
\hline $\mathrm{PN} \mathrm{G}$ & PN name & Gaia source ID & reliability & $\begin{array}{c}\text { RA } \\
(\mathrm{deg})\end{array}$ & $\begin{array}{c}\text { Dec } \\
(\mathrm{deg})\end{array}$ & $\begin{array}{c}\mathrm{G} \\
(\mathrm{mag})\end{array}$ & $\begin{array}{c}\omega \\
(\mathrm{mas})\end{array}$ & $\begin{array}{c}\sigma_{\omega} \\
\text { (mas) }\end{array}$ & $\begin{array}{c}\text { distance } \\
(\mathrm{kpc})\end{array}$ \\
\hline $197.2-14.2$ & Abell 10 & 3333922699432278656 & 1.00 & 82.94037 & +6.93389 & 18.7 & -0.060 & 0.231 & $4.48_{-099}^{+1.06}$ \\
\hline $197.4-06.4$ & WeDe 1 & 3341996555048041856 & 1.00 & 89.85361 & +10.69455 & 17.2 & 1.725 & 0.101 & $0.58_{-0.03}^{+0.84}$ \\
\hline $197.8+17.3$ & NGC 2392 & 865037169677723904 & 1.00 & 112.29486 & +20.91180 & 10.7 & 0.545 & 0.047 & $1.73_{-0.12}^{+0.14}$ \\
\hline $197.8-03.3$ & Abell 14 & 3330404266518636416 & 0.98 & 92.78607 & +11.77884 & 15.1 & 0.218 & 0.029 & $4.06_{-0.44}^{+0.55}$ \\
\hline $198.6-06.3$ & Abell 12 & 3329653849828221696 & 1.00 & 90.58333 & +9.65380 & 18.7 & 0.773 & 0.220 & $1.81_{-0.48}^{+0.65}$ \\
\hline $198.6-06.7$ & Kn 40 & 3329655597880433536 & 1.00 & 90.19664 & +9.47775 & 17.5 & 0.193 & 0.111 & \\
\hline $198.9+00.7$ & PM 1-20 & 3331504774574698752 & 1.00 & 97.01653 & +12.77247 & 19.1 & & & \\
\hline $200.5-13.1$ & Kn 63 & 3320608713128049280 & 1.00 & 85.52796 & +4.71742 & 17.5 & 0.767 & 0.095 & $1.36_{-016}^{+0.20}$ \\
\hline $200.7+08.4$ & Abell 19 & 3353841417717450112 & 0.81 & 104.98736 & +14.60947 & 12.5 & 4.137 & 0.018 & $0.24_{-0.00}^{+0.00}$ \\
\hline $202.9+07.4$ & Kn 60 & 3160750653143994880 & 1.00 & 105.02763 & +12.24479 & 19.7 & -0.215 & 0.441 & \\
\hline $204.0-08.5$ & Abell 13 & 3318052348593263488 & 1.00 & 91.19955 & +3.94319 & 19.6 & -0.002 & 0.424 & $1.98_{-043}^{+0.46}$ \\
\hline $204.4-00.4$ & StDr 13 & 3325917503163188096 & 1.00 & 98.59440 & +7.37224 & 18.1 & 0.837 & 0.162 & \\
\hline $205.0+04.4$ & CaVa 1 & 3158200679523220096 & 0.32 & 103.21414 & +9.05932 & 18.2 & 0.796 & 0.158 & \\
\hline $205.1+14.2$ & Abell 21 & 3163546505053645056 & 1.00 & 112.26128 & +13.24679 & 15.9 & 1.689 & 0.069 & $0.58_{-0.02}^{+0.02}$ \\
\hline $205.8-26.7$ & $\mathrm{MaC} 2-1$ & 3211200438511961088 & 1.00 & 75.92439 & -6.16751 & 15.8 & 0.085 & 0.080 & $12.66_{-4.00}^{+4.44}$ \\
\hline $206.4-40.5$ & NGC 1535 & 3189152962633165056 & 1.00 & 63.56572 & -12.73943 & 12.1 & 0.728 & 0.059 & $1.32_{-0.10}^{+0.11}$ \\
\hline $208.5+33.2$ & Abell 30 & 660071056749861888 & 1.00 & 131.72274 & +17.87954 & 14.4 & 0.452 & 0.033 & $2.12_{-014}^{+0.16}$ \\
\hline $208.9-07.8$ & TaWe 1 & 3121886520577421568 & 1.00 & 94.06408 & -0.00694 & 18.6 & 0.592 & 0.194 & $2.26_{-0.54}^{+0.64}$ \\
\hline $209.1-08.2$ & PHR J0615-0025 & 3121659647518519552 & 1.00 & 93.83483 & -0.43044 & 20.2 & 0.101 & 0.843 & $3.35_{-0.96}^{+0.98}$ \\
\hline $209.4+09.4$ & EGB 9 & 3154775735159860096 & 1.00 & 109.74140 & +7.37308 & 13.0 & 0.275 & 0.025 & \\
\hline $210.0+03.9$ & We 2-34 & 3128416245256794496 & 1.00 & 105.11827 & +4.34182 & 20.0 & 0.941 & 0.733 & $1.95_{-0.57}^{+0.59}$ \\
\hline $210.8-02.7$ & $\begin{array}{l}\text { IPHAS } \\
\text { J063750.80+003644.0 }\end{array}$ & 3119819065057501056 & 1.00 & 99.46169 & +0.61222 & 20.2 & -0.624 & 0.983 & -0 \\
\hline $211.2-03.5$ & M 1-6 & 3119667469890795008 & 1.00 & 98.93801 & -0.09373 & 15.5 & 0.127 & 0.044 & $5.88_{-1.00}^{+1.24}$ \\
\hline $211.4+18.4$ & HaWe 10 & 3147206692815926528 & 1.00 & 118.79710 & +9.55255 & 17.8 & 0.701 & 0.149 & $2.13^{+1.00}$ \\
\hline $212.0+04.3$ & M 1-9 & 3115628448289044992 & 1.00 & 106.33000 & +2.78318 & 15.5 & 0.111 & 0.066 & $8.54_{-210}^{+2.46}$ \\
\hline $212.6-00.0$ & PHR J0650+0013 & 3113542949610601216 & 0.32 & 102.66843 & +0.22832 & 16.5 & 0.179 & 0.064 & $4.95_{-0.95}^{+1.10}$ \\
\hline $214.9+07.8$ & Abell 20 & 3135710272253699584 & 1.00 & 110.74028 & +1.75944 & 16.4 & 0.542 & 0.057 & $1.94_{-0.20}^{+0.25}$ \\
\hline $215.2-24.2$ & IC 418 & 2985789113026163584 & 1.00 & 81.86753 & -12.69729 & 10.1 & 0.734 & 0.029 & $1.33_{-0.05}^{+0.05}$ \\
\hline $215.5-30.8$ & Abell 7 & 2986220396462236032 & 1.00 & 75.78136 & -15.60629 & 15.4 & 1.933 & 0.045 & $0.51_{-0.01}^{+0.01}$ \\
\hline $215.6+03.6$ & NGC 2346 & 3109444657456300288 & 1.00 & 107.34383 & -0.80656 & 11.2 & 0.722 & 0.018 & $1.31_{-0.03}^{+0.03}$ \\
\hline $215.6+11.1$ & Abell 22 & 3135504732298169600 & 1.00 & 114.03299 & +2.70764 & 19.4 & 1.171 & 0.393 & $2.16_{-0.83}^{+0.98}$ \\
\hline $215.7-03.9$ & BMP J0642-0417 & 3103822540968315264 & 1.00 & 100.57666 & -4.29689 & 18.8 & 1.458 & 0.272 & $0.80_{-0.14}^{+0.20}$ \\
\hline $216.0+07.4$ & PHR J0723+0036 & 3110803653827201280 & 0.98 & 110.95198 & +0.60890 & 13.8 & 0.195 & 0.022 & $4.32^{+0.43}$ \\
\hline $216.2-19.9$ & $\mathrm{~Pa} 155$ & 3009232453276290944 & 1.00 & 86.34965 & -11.76363 & 15.4 & 0.555 & 0.038 & \\
\hline $216.3-04.4$ & We $1-5$ & 3103708191756172160 & 1.00 & 100.39412 & -5.04321 & 18.6 & 0.684 & 0.209 & $.66^{+2.51}$ \\
\hline $216.9-05.2$ & MPA J0639-0554 & 3100542667721862912 & 0.99 & 99.99219 & -5.91587 & 18.9 & -0.068 & 0.284 & \\
\hline $217.1+14.7$ & Abell 24 & 3088991026757468800 & 1.00 & 117.90645 & +3.00587 & 17.4 & 1.391 & 0.097 & $0.75_{-0.05}^{+0.06}$ \\
\hline $217.2+00.9$ & PHR J0702 & 3108618546264558080 & 0.25 & 105.64345 & -3.40943 & 19.6 & 1.065 & 0.479 & $\ldots$ \\
\hline $217.4+02.0$ & St 3-1 & 3107985566868370432 & 0.96 & 106.71213 & -3.08588 & 20.8 & & & \\
\hline $218.9-10.7$ & HaWe 7 & 3001563840710096512 & 1.00 & 95.90480 & -10.22330 & 16.1 & 0.928 & 0.045 & \\
\hline $219.1+31.2$ & Abell 31 & 597324024095840512 & 1.00 & 133.55481 & +8.89800 & 15.5 & 1.842 & 0.055 & $0.54_{-0.02}^{+0.02}$ \\
\hline $219.2+07.5$ & RWT 152 & 3061893528332536192 & 1.00 & 112.49365 & -2.11045 & 13.0 & 0.265 & 0.039 & $3.62^{+0.64}$ \\
\hline $219.3+01.1$ & K 1-9 & 3053413132594418688 & 0.93 & 106.81502 & -5.16876 & 16.9 & 0.604 & 0.071 & $1.67_{-018}^{+0.23}$ \\
\hline $219.5+02.8$ & BMP J0713-0432 & 3059502709018185856 & 0.78 & 108.46255 & -4.54761 & 20.7 & & & \\
\hline $220.3-53.9$ & NGC 1360 & 5084896688945791232 & 1.00 & 53.31104 & -25.87154 & 11.2 & 2.520 & 0.065 & $0.39_{-0.01}^{+0.01}$ \\
\hline $221.0-01.4$ & PHR J0701-0749 & 3052395775097859072 & 1.00 & 105.28920 & -7.82329 & 13.1 & 0.319 & 0.015 & \\
\hline $221.3-12.3$ & IC 2165 & 2999839084924027776 & 1.00 & 95.42827 & -12.98723 & 17.5 & 0.802 & 0.343 & $2.83_{-0.92}^{+0.97}$ \\
\hline $221.6+46.4$ & EGB 6 & 615161091995252864 & 0.96 & 148.24571 & +13.74296 & 16.0 & 1.330 & 0.146 & $0.77^{+0.09}$ \\
\hline $222.1+03.9$ & PFP 1 & 3058094200264637312 & 0.60 & 110.57372 & -6.36281 & 15.8 & 1.872 & 0.074 & $0.53_{-0.02}^{+0.02}$ \\
\hline $222.5+02.9$ & WHI B & 3054938425794291072 & 1.00 & 109.91777 & -7.21978 & 20.6 & 0.207 & 0.991 & $4.52_{-1.25}^{+1.26}$ \\
\hline $222.5+07.6$ & BMP J0736-0500 & 3056940091011171456 & 1.00 & 114.09601 & -5.00567 & 20.9 & & $\ldots$ & \\
\hline $222.8-04.2$ & PM 1-23 & 3049278041153797760 & 1.00 & 103.55598 & -10.76063 & 13.9 & 0.044 & 0.016 & $9.48_{-0.97}^{+1.11}$ \\
\hline $224.3+15.3$ & Abell 25 & 3070613063558890240 & 1.00 & 121.69375 & -2.87642 & 18.4 & 0.386 & 0.163 & $3.06_{-0.68}^{+0.37}$ \\
\hline $224.3-05.5$ & PHR J0652-1240 & 2952721193446226432 & 1.00 & 103.08226 & -12.67813 & 19.7 & 0.145 & 0.440 & $1.82_{-0.40}^{+0.48}$ \\
\hline $224.9+01.0$ & We $1-6$ & 3047204259149524480 & 0.98 & 109.35852 & -10.17694 & 15.8 & 0.702 & 0.041 & $1.37_{-0.07}^{+0.08}$ \\
\hline $225.2+00.1$ & PHR J07 & 3047045306696310912 & 0.96 & 108.62018 & -10.86219 & 21.0 & & & $\ldots$ \\
\hline $225.4+00.4$ & PN We 2-37 & 47000364157035648 & 0.99 & 109.03396 & -10.88365 & 21.0 & & & \\
\hline $225.5-02.5$ & MPA J0705-1224 & 3045709473089381120 & 1.00 & 106.40511 & -12.41442 & 17.5 & -0.080 & 0.094 & $\cdots$ \\
\hline $226.4-01.3$ & PHR J0711-1238 & 3045081308353307392 & 0.51 & 107.93554 & -12.63442 & 21.2 & & & \\
\hline $226.4-03.7$ & PB 1 & 2948658394900229504 & 1.00 & 105.69484 & -13.70986 & 16.4 & 0.194 & 0.097 & $5.82_{-1.40}^{+1.59}$ \\
\hline $226.4-15.1$ & & 2943388096370398720 & 1.00 & 95.01051 & -18.62574 & 17.4 & 0.117 & 0.085 & \\
\hline $227.2-03.4$ & PHR J0705-1419 & 2948376958586005376 & 0.87 & 106.41140 & -14.31776 & 17.2 & 0.038 & 0.080 & $\ldots$ \\
\hline $227.3+12.9$ & AlvKn 1 & 3067010689807609472 & 1.00 & 121.01861 & -6.51578 & 16.1 & 1.214 & 0.077 & \\
\hline $228.2-22.1$ & LoTr 1 & 2917223705359238016 & 0.78 & 88.77753 & -22.90066 & 12.4 & 0.550 & 0.017 & $1.79_{-0.05}^{+0.06}$ \\
\hline $228.8+05.3$ & M 1-17 & 3040003385691479424 & 0.98 & 115.09239 & -11.54161 & 18.0 & & & $\ldots$ \\
\hline $229.2-36.3$ & Fr $2-3$ & 4880286371109059712 & 1.00 & 74.30576 & -28.13126 & 13.9 & 7.964 & 0.281 & \\
\hline $229.3+00.0$ & PHR J0 & 3031820167323763712 & 0.86 & 110.56220 & -14.58046 & 20.8 & & & \\
\hline $230.8+04.7$ & FP J0742-1338 & 3030427734631010304 & 0.82 & 115.56768 & -13.64186 & 20.2 & 0.595 & 0.532 & $\cdots$ \\
\hline
\end{tabular}


Table A.1: continued.

\begin{tabular}{|c|c|c|c|c|c|c|c|c|c|}
\hline PN G & PN name & Gaia source ID & reliability & $\begin{array}{c}\text { RA } \\
(\mathrm{deg})\end{array}$ & $\begin{array}{c}\text { Dec } \\
(\mathrm{deg})\end{array}$ & $\begin{array}{c}\mathrm{G} \\
(\mathrm{mag})\end{array}$ & $\begin{array}{c}\omega \\
(\mathrm{mas})\end{array}$ & $\begin{array}{c}\sigma_{\omega} \\
(\mathrm{mas})\end{array}$ & $\begin{array}{c}\text { distance } \\
(\mathrm{kpc})\end{array}$ \\
\hline $231.1+03.9$ & BMP J0739-1418 & 3030005560828868096 & 1.00 & 114.96064 & -14.30718 & 15.6 & 0.458 & 0.042 & $2.12_{-0.17}^{+0.21}$ \\
\hline $231.4+04.3$ & M 1-18 & 3029611626418190080 & 1.00 & 115.51719 & -14.35447 & 20.5 & -0.720 & 0.740 & $5.25_{-150}^{+0.55}$ \\
\hline $231.8+04.1$ & NGC 2438 & 3029214290412318720 & 1.00 & 115.46047 & -14.73540 & 17.1 & 1.383 & 0.222 & $0.84_{-0.14}^{+0.21}$ \\
\hline $232.0+05.7$ & SaSt 2-3 & 3029668728008670208 & 1.00 & 117.01530 & -14.12789 & 13.7 & 0.087 & 0.022 & $9.62_{-1.75}^{+2.54}$ \\
\hline $232.4-01.8$ & M 1-13 & 2931504604759238912 & 0.83 & 110.31218 & -18.14337 & 19.5 & -0.680 & 0.559 & $4.23_{-1.04}^{+1.08}$ \\
\hline $232.6-01.0$ & PHR J0724-1757 & 3027558047942245376 & 1.00 & 111.18050 & -17.96138 & 20.9 & & & \\
\hline $232.8-04.7$ & M 1-11 & 2931008523160833280 & 1.00 & 107.81956 & -19.85079 & 13.8 & 0.217 & 0.028 & $3.98_{-0.38}^{+0.46}$ \\
\hline $233.0+03.5$ & StDr 20 & 3028738476748576768 & 1.00 & 115.57857 & -16.14476 & 17.8 & 0.851 & 0.097 & \\
\hline $233.0-10.1$ & SaWe 1 & 2925763887047836416 & 1.00 & 102.66964 & -22.43652 & 20.6 & 0.317 & 0.699 & \\
\hline $233.5-16.3$ & Abell 15 & 2924036760440812160 & 1.00 & 96.75851 & -25.38045 & 15.9 & 0.166 & 0.042 & $5.90_{-1.01}^{+1.26}$ \\
\hline $234.3-07.2$ & MPA J0704-2221 & 2928626633072247680 & 0.99 & 106.09575 & -22.36442 & 19.4 & 0.654 & 0.318 & $2.33_{-0.64}^{+0.70}$ \\
\hline $234.8+02.4$ & NGC 2440 & 5716990599321527552 & 0.51 & 115.48057 & -18.20864 & 17.5 & -1.473 & 0.451 & $1.76_{-028}^{+0.30}$ \\
\hline $234.9-01.4$ & M 1-14 & 5620439768860185856 & 1.00 & 111.98545 & -20.22295 & 14.3 & 0.097 & 0.036 & $6.11_{-0.80}^{+0.99}$ \\
\hline $234.9-09.7$ & MPA J0656-2356 & 2922355602864621568 & 1.00 & 103.99977 & -23.94705 & 19.1 & 0.322 & 0.199 & $2.93_{-0.64}^{+0.91}$ \\
\hline $235.3-03.9$ & M 1-12 & 2929602385226601344 & 1.00 & 109.83945 & -21.73205 & 14.0 & 0.219 & 0.028 & $4.09_{-0.43}^{+0.55}$ \\
\hline $235.7+07.1$ & PHR J0800-1635 & 5718155802460841472 & 1.00 & 120.24626 & -16.59371 & 19.6 & -0.109 & 0.353 & $3.46_{-0.80}^{+0.85}$ \\
\hline $235.9+23.2$ & Pa 156 & 5756008831004996864 & 1.00 & 134.21994 & -8.06505 & 16.7 & 1.169 & 0.065 & \\
\hline $236.0-10.6$ & HaWe 9 & 2921083334772992896 & 1.00 & 103.58666 & -25.40933 & 19.6 & 0.557 & 0.297 & $1.93_{-0.45}^{+0.50}$ \\
\hline $236.9+08.6$ & PHR J0809-1650 & 5721124453798128640 & 1.00 & 122.25611 & -16.83391 & 17.6 & 0.273 & 0.104 & \\
\hline $237.0+00.7$ & PHR J0740-2055 & 5715387335262528640 & 0.91 & 115.09539 & -20.93177 & 18.1 & 0.655 & 0.122 & $1.79^{+}$ \\
\hline $237.3-08.4$ & BMP J0705-2528 & 5611419577328099840 & 1.00 & 106.43973 & -25.48043 & 18.7 & 0.560 & 0.148 & $3.76_{-1}^{+1}$ \\
\hline 237.4-09.6 & BMP J0700-2607 & 2920762925913505024 & 1.00 & 105.21587 & -26.12179 & 19.0 & 0.718 & 0.182 & $2.31_{-0.68}^{+0.39}$ \\
\hline $238.0+34.8$ & Abell 33 & 3827045525522912128 & 0.93 & 144.78802 & -2.80843 & 15.9 & 1.005 & 0.059 & $1.00_{-0.06}^{+0.06}$ \\
\hline $238.9+07.3$ & Sa $2-21$ & 5713819779576751488 & 1.00 & 122.18439 & -19.23381 & 19.5 & 0.140 & 0.396 & $4.38_{-1.19}^{+1.23}$ \\
\hline $239.6+13.9$ & NGC 2610 & 5709928951521751168 & 1.00 & 128.34757 & -16.14936 & 15.9 & 0.578 & 0.060 & $1.77_{-0.17}^{+0.21}$ \\
\hline $239.6-12.0$ & ESO 427-19 & 5608770995551017600 & 1.00 & 103.80099 & -29.12468 & 20.2 & 0.293 & 0.454 & $\begin{array}{l}7.07_{-2.06}^{+2.07} \\
\text { - }\end{array}$ \\
\hline $240.1-01.0$ & $\mathrm{~Pa} 60$ & 5615067658135032960 & 1.00 & 115.04359 & -24.49758 & 16.3 & 0.218 & 0.046 & \\
\hline $240.3+07.0$ & Y-C 2-5 & 5701379385324474240 & 1.00 & 122.67350 & -20.52564 & 15.6 & 0.154 & 0.046 & \\
\hline $240.3-07.6$ & M 3-2 & 5609860130542365824 & 0.58 & 108.70801 & -27.83978 & 16.3 & 0.077 & 0.041 & $9.86_{-205}^{+2.40}$ \\
\hline $240.6-07.7$ & BMP J0715-2805 & 5609834734391604736 & 1.00 & 108.75948 & -28.09558 & 18.1 & 0.200 & 0.105 & \\
\hline $241.0+02.3$ & M 3-4 & 5710725616423348352 & 1.00 & 118.79749 & -23.63683 & 15.2 & 0.270 & 0.028 & $3.35_{-0.29}^{+0.35}$ \\
\hline $242.3-02.4$ & FP J0739-2709 & 5612254965642151808 & 1.00 & 114.90882 & -27.15841 & 18.9 & 0.329 & 0.168 & $1.72_{-0.24}^{+0.26}$ \\
\hline $242.6-04.4$ & PHR J0732-2825 & 5611737443573911168 & 0.86 & 113.07313 & -28.42162 & 18.4 & -0.036 & 0.127 & \\
\hline $242.6-11.6$ & M 3-1 & 5604523410329326720 & 0.78 & 105.70792 & -31.59143 & 15.6 & -0.014 & 0.047 & $6.73_{-0.85}^{+0.93}$ \\
\hline $243.0+36.5$ & WPS 75 & 3822583947855569280 & 1.00 & 148.59553 & -5.03607 & 18.2 & 2.492 & 0.156 & \\
\hline $243.3-01.0$ & NGC 2452 & 5601582285451511040 & 0.98 & 116.85942 & -27.33521 & 17.2 & 0.342 & 0.079 & $3.13_{-0.54}^{+0.67}$ \\
\hline $243.8-37.1$ & PRTM 1 & 4814231938760137856 & 1.00 & 75.75722 & -39.76237 & 15.5 & 0.130 & 0.035 & $7.78_{-140}^{+1.73}$ \\
\hline $244.2+06.2$ & PHR J0816-2410 & 5699342883632418432 & 0.97 & 124.22171 & -24.18194 & 20.8 & 1.773 & 1.283 & \\
\hline $244.5+12.5$ & Abell 29 & 5703415268543295744 & 1.00 & 130.07883 & -20.91015 & 18.2 & 0.940 & 0.127 & $1.11_{-0.13}^{+0.15}$ \\
\hline $245.0+02.2$ & BMP J0803-2706 & 5697057243772021120 & 1.00 & 120.97602 & -27.10053 & 20.6 & -0.567 & 0.795 & $2.44_{-0.61}^{+0.64}$ \\
\hline $245.1-05.5$ & BMP J0733-3108 & 5593164110894267904 & 1.00 & 113.35038 & -31.13458 & 18.4 & 0.851 & 0.118 & $1.19_{-013}^{+0.15}$ \\
\hline $247.5-04.7$ & HFG 2 & 5594969135329315072 & 1.00 & 115.59902 & -32.79746 & 17.0 & 0.528 & 0.052 & $1.91_{-017}^{+0.20}$ \\
\hline $247.8+04.9$ & FP J0821-2755 & 5693172325603777408 & 0.97 & 125.32640 & -27.92669 & 17.4 & 0.132 & 0.072 & $3.62_{-0.47}^{+0.52}$ \\
\hline $248.0-05.3$ & Dr 2 & 5588907974395422720 & 0.99 & 115.22935 & -33.55665 & 20.4 & 0.058 & 0.540 & \\
\hline $248.5+10.5$ & PHR J0843-2514 & 5653202604024938880 & 1.00 & 130.83248 & -25.24118 & 19.4 & 0.318 & 0.349 & $5.54_{-1.55}^{+1.58}$ \\
\hline $248.7+29.5$ & Abell 34 & 5690534730341025408 & 1.00 & 146.39725 & -13.17111 & 16.4 & 0.845 & 0.066 & $1.22_{-0.09}^{+0.31}$ \\
\hline $248.8-08.5$ & M 4-2 & 5589719276543497216 & 1.00 & 112.22426 & -35.75388 & 18.8 & 0.957 & 0.220 & $1.79_{-0.69}^{+3.14}$ \\
\hline $249.3-05.4$ & Abell 23 & 5588562590306924416 & 0.99 & 115.82585 & -34.75310 & 20.5 & 0.167 & 0.623 & $3.00_{-0.82}^{+0.85}$ \\
\hline $249.8+07.1$ & PHR J0834-2819 & 5645669433248890496 & 1.00 & 128.57531 & -28.31760 & 18.8 & 0.257 & 0.162 & $3.43_{-0.76}^{+0.86}$ \\
\hline $250.5+01.9$ & BMP J0816-3150 & 5547940441729900800 & 0.89 & 124.08688 & -31.84989 & 19.7 & -0.175 & 0.352 & \\
\hline $250.6+09.3$ & BMP J0844-2737 & 5645989184974881536 & 1.00 & 131.15768 & -27.62070 & 19.3 & 0.173 & 0.266 & $4.08_{-0.99}^{+1.05}$ \\
\hline $252.5-01.6$ & Pre 50 & 5544890332054286208 & 0.99 & 121.81582 & -35.44412 & 17.8 & 0.073 & 0.078 & \\
\hline $253.5+10.7$ & K 1-2 & 5647809392112960000 & 1.00 & 134.44150 & -28.96015 & 17.0 & 0.358 & 0.073 & $3.02_{-0.52}^{+0.68}$ \\
\hline $253.9+05.7$ & M 3-6 & 5639472001599302528 & 1.00 & 130.16761 & -32.37598 & 13.2 & 0.346 & 0.033 & $2.68_{-0.22}^{+0.26}$ \\
\hline $254.7-18.2$ & Fr 2-24 & 5558802624472332928 & 1.00 & 103.61871 & -44.97574 & 15.3 & 0.398 & 0.019 & $2.06_{-0.07}^{+0.07}$ \\
\hline $255.3-03.6$ & WRAY 15-158 & 5540866394373376384 & 0.97 & 121.61802 & -38.89009 & 17.0 & -0.226 & 0.264 & \\
\hline $255.3-59.6$ & Lo 1 & 4755520010701141504 & 1.00 & 44.24360 & -44.17156 & 15.1 & 1.238 & 0.039 & $0.80_{-0.03}^{+0.03}$ \\
\hline $255.7+03.3$ & Wray $16-22$ & 5638577690630389888 & 0.96 & 129.06882 & -35.25053 & 20.9 & & & \\
\hline $255.8+10.9$ & FP J0905-3033 & 5635240088724202496 & 1.00 & 136.27225 & -30.55336 & 16.4 & 1.300 & 0.048 & $0.77_{-0.03}^{+0.03}$ \\
\hline $257.8-05.4$ & KLSS $1-10$ & 5533596865866854144 & 0.83 & 121.39033 & -41.93792 & 20.5 & 2.090 & 1.469 & \\
\hline $258.0-03.2$ & BMP J0815-4053 & 5539487911728155520 & 1.00 & 123.98685 & -40.88548 & 20.0 & 0.046 & 0.360 & \\
\hline $258.0-15.7$ & Lo 3 & 5509004952576699904 & 1.00 & 108.70594 & -46.96087 & 16.7 & 0.467 & 0.074 & $2.18_{-0.28}^{+0.35}$ \\
\hline $258.1-00.3$ & Hen 2-9 & 5528078623162786688 & 1.00 & 127.11648 & -39.39444 & 16.3 & 0.203 & 0.051 & $4.00_{-0.54}^{+0.60}$ \\
\hline $258.5-01.3$ & RCW 24 & 5527934587141415168 & 1.00 & 126.44808 & -40.21942 & 18.2 & 1.186 & 0.102 & $0.83_{-0.06}^{+0.84}$ \\
\hline $259.1+00.9$ & Hen $2-11$ & 5528673875570999680 & 1.00 & 129.28478 & -39.41896 & 17.5 & 0.611 & 0.071 & $1.37_{-0.10}^{+0.00}$ \\
\hline $259.2+02.9$ & St 45 & 5528999017473732736 & 1.00 & 131.32454 & -38.30306 & 19.7 & 1.021 & 0.295 & $\ldots$ \\
\hline $259.6+13.2$ & $\mathrm{~Pa} 163$ & 5631349393807930752 & 1.00 & 141.23000 & -31.75046 & 17.8 & 0.482 & 0.102 & \\
\hline $260.9-08.5$ & Dr 1 & 5519032361187182848 & 1.00 & 119.95456 & -46.17954 & 19.6 & 0.428 & 0.295 & $\ldots$ \\
\hline
\end{tabular}


Table A.1: continued.

\begin{tabular}{|c|c|c|c|c|c|c|c|c|c|}
\hline PN G & PN name & Gaia source ID & reliability & $\begin{array}{c}\text { RA } \\
(\text { deg) }\end{array}$ & $\begin{array}{c}\text { Dec } \\
(\mathrm{deg})\end{array}$ & $\begin{array}{c}\mathrm{G} \\
(\mathrm{mag})\end{array}$ & $\begin{array}{c}\omega \\
\text { (mas) }\end{array}$ & $\begin{array}{c}\sigma_{\omega} \\
(\mathrm{mas})\end{array}$ & $\begin{array}{c}\text { distance } \\
(\mathrm{kpc})\end{array}$ \\
\hline $261.0+32.0$ & NGC 3242 & 5668874905325843456 & 1.00 & 156.19220 & -18.64230 & 12.2 & 0.745 & 0.050 & $1.27_{-0.07}^{+0.08}$ \\
\hline $261.2-04.6$ & PHR J0819-4423 & 5520199316680704128 & 0.31 & 124.83183 & -44.38491 & 19.5 & 0.655 & 0.323 & \\
\hline $261.6+03.0$ & Hen $2-15$ & 5525558890406420224 & 0.94 & 133.37867 & -40.06188 & 21.3 & & & \\
\hline $261.9+08.5$ & NGC 2818 & 5623380240906854656 & 0.30 & 139.00619 & -36.62707 & 19.0 & 0.032 & 0.211 & $2.64_{-047}^{+0.52}$ \\
\hline $261.9-05.3$ & BMP J0818-4517 & 5519846442169441408 & 1.00 & 124.57008 & -45.29912 & 19.9 & 1.073 & 0.387 & \\
\hline $262.6-04.6$ & Wray $17-18$ & 5516878589711567360 & 1.00 & 125.97416 & -45.51927 & 20.2 & 0.741 & 0.481 & $5.22_{-183}^{+1.82}$ \\
\hline $263.0-05.5$ & PB 2 & 5516695417937365376 & 0.91 & 125.16744 & -46.38291 & 15.8 & 0.251 & 0.066 & $5.44_{-1.162}^{+2.83}$ \\
\hline $264.1-08.1$ & Hen 2-7 & 5516068971190925184 & 0.97 & 122.88287 & -48.72138 & 17.2 & 0.170 & 0.085 & $4.02_{-0.65}^{+0.73}$ \\
\hline $264.4-12.7$ & Hen 2-5 & 5492948986137201664 & 1.00 & 116.83350 & -51.25094 & 14.7 & 0.088 & 0.042 & $7.64_{-1.131}^{+1.52}$ \\
\hline $264.5+05.0$ & FP J0911-4051 & 5428634329076290944 & 1.00 & 137.94013 & -40.86624 & 18.9 & 1.014 & 0.160 & \\
\hline $264.6+03.8$ & BMP J0907-4146 & 5428189503611241984 & 1.00 & 136.85124 & -41.77053 & 18.9 & 1.120 & 0.155 & $0.98_{-013}^{+0.17}$ \\
\hline $265.1-02.2$ & Hen 2-13 & 5329858293258344448 & 1.00 & 130.86707 & -46.11110 & 18.3 & & & \\
\hline $265.1-04.2$ & LoTr 3 & 5521499734013832448 & 1.00 & 128.52842 & -47.27736 & 20.6 & 0.506 & 1.013 & $4.45_{-137}^{+1.37}$ \\
\hline $265.4+04.2$ & PHR J0911-4205 & 5427382290279252736 & 0.48 & 137.95240 & -42.08628 & 19.9 & 0.850 & 0.347 & $\ldots$ \\
\hline $265.6+09.5$ & MPA J0932-3827 & 5429861276680613248 & 1.00 & 143.07440 & -38.45799 & 19.0 & -0.035 & 0.192 & \\
\hline $265.7+04.1$ & NGC 2792 & 5427326253844953216 & 1.00 & 138.11090 & -42.42773 & 16.7 & 0.397 & 0.058 & $2.58_{-0.32}^{+0.41}$ \\
\hline $268.2+03.1$ & PHR J0918-4454 & 5423882514704132096 & 0.36 & 139.50650 & -44.91056 & 21.0 & & & \\
\hline $268.9-00.4$ & BRAN 229 & 5327072405662519808 & 0.68 & 136.42096 & -47.90141 & 12.1 & 0.647 & 0.011 & \\
\hline $269.7-03.6$ & PB 3 & 5324856717933777792 & 0.98 & 133.57649 & -50.53961 & 20.1 & & & \\
\hline $270.1+24.8$ & K $1-28$ & 5455506530699796608 & 1.00 & 158.62755 & -29.18758 & 16.5 & 0.490 & 0.067 & $2.28_{-0.32}^{+0.45}$ \\
\hline $270.1-02.9$ & Wray $17-23$ & 5325030612573630464 & 0.97 & 134.76218 & -50.39451 & 20.3 & & & \\
\hline $272.1+12.3$ & NGC 3132 & 5420219732228461184 & 0.81 & 151.75731 & -40.43643 & 10.0 & 1.320 & 0.034 & $0.75_{-0.02}^{+0.02}$ \\
\hline $272.4-05.9$ & MeWe 1-1 & 5317493563454092288 & 1.00 & 133.40345 & -54.08562 & 19.1 & -0.028 & 0.239 & $2.61_{-0.48}^{+0.52}$ \\
\hline $272.8+01.0$ & PHR J0928-4936 & 5313911079687293440 & 1.00 & 142.17070 & -49.61296 & 16.9 & 0.411 & 0.056 & $\ldots .40$ \\
\hline $272.8-02.3$ & PM 1-47 & 5311937623810283776 & 1.00 & 138.38765 & -52.04489 & 20.1 & & & $\ldots$ \\
\hline $272.9-21.7$ & Kn 69 & 5286831276515953664 & 1.00 & 107.42271 & -62.23992 & 17.7 & 0.231 & 0.076 & $\ldots$ \\
\hline $273.2-00.3$ & PHR J0924 & 5313376648321659264 & 0.39 & 141.02787 & -50.89056 & 20.0 & -0.196 & 0.378 & 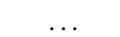 \\
\hline $273.2-03.7$ & Hen 2-18 & 5311360105332008448 & 0.99 & 137.16688 & -53.32050 & 20.9 & & & $\ldots$ \\
\hline $273.4+00.7$ & $\mathrm{~Pa} 168$ & 5313831944902282880 & 0.98 & 142.60424 & -50.27523 & 21.0 & & & \\
\hline $273.6+06.1$ & HbDs 1 & 5411328565822822528 & 1.00 & 148.18549 & -46.27978 & 12.4 & 1.341 & 0.042 & . \\
\hline $274.2-09.7$ & Fr 2-6 & 5314960460454288768 & 0.25 & 130.06838 & -57.91238 & 14.7 & 1.017 & 0.032 & \\
\hline $274.3+09.1$ & Lo 4 & 5414927915911816704 & 1.00 & 151.44074 & -44.35931 & 16.6 & 0.330 & 0.052 & $3.49_{-056}^{+0.79}$ \\
\hline $274.6+02.1$ & Hen 2-35 & 5409038794190643840 & 0.96 & 145.40621 & -49.96626 & 16.0 & 0.090 & 0.048 & $8.49_{-171}^{+2.08}$ \\
\hline $274.6+03.5$ & Hen 2-37 & 5409429846673479424 & 1.00 & 146.85330 & -48.97073 & 20.6 & 2.145 & 0.851 & $3.63_{-145}^{+1.33}$ \\
\hline $274.8-04.2$ & MPA J0913-5445 & 5310271421338065024 & 0.98 & 138.33598 & -54.76019 & 20.0 & 0.176 & 0.401 & \\
\hline $275.0-04.1$ & PB 4 & 5310278598198854016 & 1.00 & 138.78224 & -54.87883 & 16.3 & 0.191 & 0.045 & $4.51^{+0.74}$ \\
\hline $275.1-03.5$ & PHR J0918-5431 & 5310620069579969664 & 0.36 & 139.61061 & -54.52651 & 18.0 & 0.018 & 0.104 & \\
\hline $275.2-02.9$ & Hen 2-28 & 5310734178295067008 & 0.91 & 140.52821 & -54.16094 & 16.5 & 0.186 & 0.044 & $5.35_{-0.91}^{+1.16}$ \\
\hline $275.5-01.3$ & Pe 2-4 & 5312206939763090304 & 0.97 & 142.70158 & -53.16653 & 20.0 & -0.803 & 0.730 & $4.32_{-1.29}^{+1.36}$ \\
\hline $275.7-04.9$ & PHR J0914-5555 & 5310074162075205248 & 0.98 & 138.51469 & -55.92230 & 16.5 & 0.108 & 0.037 & $\ldots$ \\
\hline $275.8-02.9$ & Hen 2-29 & 5310519051961769984 & 0.99 & 141.19098 & -54.60431 & 21.0 & & & $\ldots$ \\
\hline $276.1-11.9$ & MPA J0835-6039 & 5302075627283541760 & 1.00 & 128.78036 & -60.66185 & 17.0 & 0.129 & 0.047 & \\
\hline $276.2-06.6$ & PHR J0907-5722 & 5303880196458455808 & 0.38 & 136.96246 & -57.38130 & 18.7 & 0.703 & 0.152 & $1.60_{-027}^{+0.34}$ \\
\hline $276.5+03.1$ & MPA J0954-5026 & 5406067501454862592 & 1.00 & 148.65607 & -50.44912 & 19.6 & 0.045 & 0.289 & \\
\hline $277.1+03.3$ & MeWe 2-2 & 5405299634331490944 & 0.73 & 149.54284 & -50.65941 & 20.7 & & & $\ldots$ \\
\hline $277.1-01.5$ & Wray $16-55$ & 5308757256383765504 & 0.83 & 144.46587 & -54.45240 & 18.1 & -0.088 & 0.282 & \\
\hline $277.1-03.8$ & NGC 2899 & 5307241888842877696 & 0.61 & 141.76315 & -56.10570 & 15.9 & 0.446 & 0.028 & $2.03_{-0.10}^{+0.12}$ \\
\hline $277.2-03.0$ & PHR J0931-5535 & 5307358711931763456 & 0.25 & 142.85857 & -55.59527 & 21.0 & & & -0 \\
\hline $277.2-36.0$ & RP 4785 & 4662151269979497216 & 0.56 & 74.22729 & -66.47339 & 20.5 & 3.825 & 0.737 & \\
\hline $277.7-03.5$ & Wray $17-31$ & 5307054800062198400 & 1.00 & 142.83547 & -56.29431 & 17.9 & 0.736 & 0.084 & $1.44_{-0.15}^{+0.19}$ \\
\hline $278.1-05.9$ & NGC 2867 & 5300450617836957312 & 0.99 & 140.35572 & -58.31127 & 15.8 & 0.345 & 0.056 & $2.61_{-029}^{+0.34}$ \\
\hline $278.2-33.4$ & HD 269404 & 4658744330153413760 & 1.00 & 80.37383 & -67.85189 & 14.8 & -1.577 & 0.355 & \\
\hline $278.3-04.3$ & PHR J0930-5716 & 5306569636249042944 & 0.71 & 142.70513 & -57.27235 & 19.0 & 0.581 & 0.226 & $\ldots$ \\
\hline $278.7-28.9$ & RP 4791 & 5282784004280482816 & 0.98 & 92.36173 & -68.65654 & 20.2 & -0.268 & 0.372 & \\
\hline $278.8+04.9$ & PB 6 & 5358679035649615232 & 1.00 & 153.31648 & -50.33320 & 17.1 & 0.149 & 0.064 & $5.93_{-1.13}^{+1.32}$ \\
\hline $279.0+00.6$ & PHR J0957-5357 & 5308313122421945472 & 0.82 & 149.35980 & -53.95239 & 16.4 & 0.261 & 0.040 & \\
\hline $279.1-00.4$ & IRAS 09517-5438 & 5308167437119835136 & 0.99 & 148.36275 & -54.87773 & 20.4 & -0.483 & 0.798 & $\ldots$ \\
\hline $279.6+02.2$ & PHR J1007-5304 & 5356821582547892352 & 0.92 & 151.80082 & -53.07903 & 18.6 & 0.253 & 0.144 & \\
\hline $279.6-03.1$ & Hen 2-36 & 5306043043221261568 & 1.00 & 145.85675 & -57.28207 & 11.2 & 0.246 & 0.013 & $3.70_{-0.16}^{+0.17}$ \\
\hline $280.0+02.9$ & Sa 2-56 & 5356894356478901760 & 1.00 & 152.99033 & -52.63806 & 17.7 & 0.201 & 0.088 & $6.26_{-1.57}^{+1.80}$ \\
\hline $280.1-05.1$ & BMP J0936-5905 & 5305458103016583552 & 1.00 & 144.18165 & -59.08801 & 20.8 & -0.751 & 1.270 & $3.00_{-0.80}^{+0.81}$ \\
\hline $281.0-05.6$ & IC 2501 & 5257233312554459392 & 0.86 & 144.69659 & -60.09187 & 14.4 & 0.133 & 0.054 & $4.12_{-0.50}^{+0.56}$ \\
\hline $282.6-00.4$ & PM 1-51 & 5258954533593291136 & 1.00 & 153.33198 & -56.92563 & 20.8 & & & $\ldots$ \\
\hline $282.9+03.8$ & Hen $2-48$ & 5357078902646466432 & 0.99 & 157.88328 & -53.55846 & 19.6 & -0.392 & 0.451 & \\
\hline $283.3-03.4$ & PHR1004-5946 & 5256731110621038336 & 0.88 & 151.17454 & -59.78092 & 17.4 & 0.082 & 0.069 & $\ldots$ \\
\hline $283.4+01.1$ & PHR J1024-5600 & 5354962720694112768 & 0.96 & 156.14092 & -56.00881 & 20.3 & 0.899 & 0.558 & $\ldots$ \\
\hline $283.4+02.8$ & PHR J1030-5437 & 5352520220035609600 & 1.00 & 157.73224 & -54.63287 & 20.3 & 0.851 & 0.506 & \\
\hline $283.4-01.3$ & MeWe 1-2 & 5258596741332679040 & 1.00 & 153.60030 & -58.19776 & 18.8 & 0.425 & 0.187 & $1.75_{-0.28}^{+0.32}$ \\
\hline $283.6+25.3$ & K $1-22$ & 5399388969045865344 & 0.83 & 171.68244 & -34.36970 & 16.7 & 0.724 & 0.461 & $1.38_{-0.32}^{+0.35}$ \\
\hline $283.7-05.1$ & PHR J0958-6126 & 5256159162714165376 & 0.84 & 149.63393 & -61.44519 & 17.6 & 0.122 & 0.069 & $\ldots$ \\
\hline
\end{tabular}


Table A.1: continued.

\begin{tabular}{|c|c|c|c|c|c|c|c|c|c|}
\hline PN G & PN name & Gaia source ID & reliability & $\begin{array}{c}\text { RA } \\
(\mathrm{deg})\end{array}$ & $\begin{array}{c}\text { Dec } \\
(\mathrm{deg})\end{array}$ & $\begin{array}{c}\mathrm{G} \\
(\mathrm{mag})\end{array}$ & $\begin{array}{c}\omega \\
(\mathrm{mas})\end{array}$ & $\begin{array}{c}\sigma_{\omega} \\
\text { (mas) }\end{array}$ & $\begin{array}{c}\text { distance } \\
(\mathrm{kpc})\end{array}$ \\
\hline $283.8+02.2$ & My 60 & 5352413567402723328 & 1.00 & 157.88927 & -55.34743 & 17.9 & 0.406 & 0.131 & $3.42_{-0.88}^{+1.08}$ \\
\hline $283.8-04.2$ & Hen 2-39 & 5256396485463285504 & 0.93 & 150.95497 & -60.73006 & 15.7 & 0.068 & 0.030 & $8.05_{-111}^{+1.89}$ \\
\hline $283.9+09.7$ & DS 1 & 5362804330246457344 & 1.00 & 163.66888 & -48.78408 & 12.1 & 1.226 & 0.031 & $0.79_{-0.02}^{+0.02}$ \\
\hline $284.2-05.3$ & PHR J1001-6152 & 5255947536784791936 & 1.00 & 150.32822 & -61.86771 & 16.7 & 0.268 & 0.048 & \\
\hline $284.4+07.8$ & PHR J1052-5042 & 5360264943724607360 & 0.32 & 163.11089 & -50.69953 & 16.3 & 0.470 & 0.038 & \\
\hline $284.5+03.8$ & PHR J1040-5417 & 5353877945092382336 & 1.00 & 160.18824 & -54.30347 & 16.6 & 0.406 & 0.047 & $2.34_{-0202}^{+0.26}$ \\
\hline $284.6-04.5$ & PHR J1007-6124 & 5256301476452488320 & 0.88 & 151.96390 & -61.40672 & 19.8 & 0.022 & 0.393 & \\
\hline $285.4+01.2$ & Pe $1-2$ & 5351224999667770880 & 0.98 & 159.88620 & -57.10391 & 19.2 & 1.235 & 0.608 & $4.44_{-2.67}^{+2.89}$ \\
\hline $285.4+01.5$ & Pe 1-1 & 5352007199082209792 & 0.83 & 159.61494 & -56.78534 & 17.9 & & & \\
\hline $285.4-05.3$ & IC 2553 & 5252805514840414592 & 0.99 & 152.33688 & -62.61347 & 16.2 & 0.268 & 0.124 & $3.48_{-070}^{+0.80}$ \\
\hline $285.4-12.2$ & Kn 70 & 5246931889329817344 & 1.00 & 141.78244 & -67.85693 & 20.4 & 0.733 & 0.531 & \\
\hline $285.5-03.3$ & BRAN 303 & 5254677364660203648 & 0.69 & 154.86439 & -60.98622 & 18.0 & 0.282 & 0.119 & \\
\hline $285.6-02.7$ & Мy 59 & 5254793942926363392 & 0.99 & 155.78805 & -60.54503 & 12.9 & 0.223 & 0.023 & $4.22_{-036}^{+0.43}$ \\
\hline $285.7-14.9$ & IC 2448 & 5222772389050179840 & 1.00 & 136.77625 & -69.94183 & 14.2 & 0.280 & 0.032 & $3.18_{-027}^{+0.31}$ \\
\hline $286.0-06.5$ & Hen 2-41 & 5252614990078889216 & 1.00 & 151.84841 & -63.90835 & 15.5 & & & \\
\hline $286.0-19.9$ & Kn 71 & 5220864014824048384 & 1.00 & 124.17697 & -72.99339 & 17.9 & 0.124 & 0.088 & .. \\
\hline $286.1-02.0$ & MPA J1029-6014 & 5255143179642882304 & 0.99 & 157.28156 & -60.23428 & 18.8 & -0.029 & 0.172 & \\
\hline $286.2-06.9$ & Wray $17-40$ & 5246408689299887488 & 1.00 & 151.74826 & -64.36384 & 16.5 & 0.388 & 0.044 & $2.60_{-0.25}^{+0.30}$ \\
\hline $286.3+02.8$ & Hen 2-55 & 5352847947540781568 & 1.00 & 162.18008 & -56.05267 & 17.0 & 0.229 & 0.059 & $4.42_{-0.86}^{+1.166}$ \\
\hline $286.3-03.1$ & PHR J1025-6115 & 5254617681813177472 & 0.56 & 156.44257 & -61.25654 & 17.2 & 0.287 & 0.057 & \\
\hline $286.3-04.8$ & NGC 3211 & 5252991607174388736 & 0.97 & 154.45965 & -62.67069 & 17.5 & 0.413 & 0.099 & $2.81_{-054}^{+0.68}$ \\
\hline $286.4-30.9$ & & 4648624798759687040 & 1.00 & 84.12871 & -75.11777 & 19.8 & 0.069 & 0.296 & \\
\hline $286.5+11.6$ & Lo 5 & 5374348102823679744 & 1.00 & 168.47573 & -47.95008 & 16.9 & 0.695 & 0.064 & $1.50_{-0.13}^{+0.16}$ \\
\hline $286.8-29.5$ & K 1-27 & 4648434175230114048 & 1.00 & 89.25908 & -75.67288 & 16.0 & 0.253 & 0.035 & $4.18_{-0.52}^{+0.67}$ \\
\hline $288.2+00.4$ & MPA J1053-5903 & 5338812711647438848 & 0.91 & 163.38315 & -59.05072 & 19.5 & 0.449 & 0.257 & \\
\hline $288.4+00.3$ & Hf 38 & 5338808043069762816 & 0.44 & 163.64649 & -59.16297 & 20.0 & -0.896 & 0.531 & $2.66_{-0.61}^{+0.65}$ \\
\hline $288.7+08.1$ & ESO 216-2 & 5348444781415074432 & 1.00 & 169.54068 & -52.16739 & 15.6 & 0.277 & 0.036 & $3.62_{-0.44}^{+0.57}$ \\
\hline $288.8-05.2$ & Hen 2-51 & 5251802141752703360 & 0.49 & 158.94042 & -64.31987 & 15.1 & 0.275 & 0.027 & $3.32_{-0.27}^{+0.43}$ \\
\hline $289.0+03.3$ & PHR J1107-5642 & 5340787645002807424 & 1.00 & 166.92854 & -56.70751 & 20.0 & -0.535 & 0.388 & $2.15_{-0.41}^{+0.44}$ \\
\hline $289.5-03.8$ & MPA J1046-6328 & 5241401655115772800 & 1.00 & 161.69054 & -63.46975 & 17.2 & -0.009 & 0.057 & -0.41 \\
\hline $289.7+06.9$ & MPA J1121-5339 & 5346800736642214144 & 0.90 & 170.47306 & -53.65142 & 18.3 & 0.380 & 0.116 & 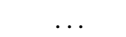 \\
\hline $289.8+07.7$ & Hen 2-63 & 5348165093140615808 & 0.55 & 171.00434 & -52.85521 & 19.1 & & & . \\
\hline $290.1-02.5$ & MPA J1057-6233 & 5241688387109076608 & 0.89 & 164.29036 & -62.56129 & 19.9 & 0.356 & 0.348 & \\
\hline $290.5+07.9$ & Fg 1 & 5348195948185156736 & 1.00 & 172.15084 & -52.93445 & 14.5 & 0.391 & 0.027 & $2.38_{-0.14}^{+0.15}$ \\
\hline $290.8+00.5$ & Pre 43 & 5337772681029635840 & 0.99 & 168.11382 & -59.97729 & 20.8 & 1.044 & 1.384 & -0. \\
\hline $290.9-00.6$ & DeGaPe 27 & 5337295660486717568 & 0.61 & 167.35721 & -61.06934 & 19.9 & 0.624 & 0.421 & $\ldots$ \\
\hline $291.3+03.7$ & PHR J1124-5711 & 5339786505301392256 & 0.26 & 171.21139 & -57.18573 & 20.9 & & & \\
\hline $291.3+08.4$ & PHR J1134-5243 & 5345597458614614400 & 1.00 & 173.66061 & -52.72555 & 13.0 & 0.040 & 0.018 & $9.73_{-1.05}^{+1.18}$ \\
\hline $291.4+19.2$ & LoTr 4 & 5380413425574962944 & 1.00 & 178.12186 & -42.29416 & 16.5 & 0.147 & 0.066 & $6.96_{-1.53}^{+1.79}$ \\
\hline $291.4-00.3$ & NDK 1 & 5337430973471180160 & 0.40 & 168.63304 & -61.00064 & 17.6 & 0.050 & 0.077 & $\ldots$ \\
\hline $291.6-04.8$ & IC 2621 & 5240291148370061952 & 0.93 & 165.08366 & -65.24948 & 14.5 & & & \\
\hline $291.7+03.7$ & Hen 2-64 & 5342772538383389824 & 1.00 & 171.85106 & -57.29969 & 15.5 & 0.225 & 0.071 & $5.62_{-1.54}^{+2.13}$ \\
\hline $292.3+00.5$ & PHR J1123-6030 & 5337515429719994240 & 0.72 & 170.96294 & -60.51382 & 17.9 & & & \\
\hline $292.4+04.1$ & PB 8 & 5342835824697866880 & 0.95 & 173.32417 & -57.10414 & 14.3 & 0.170 & 0.026 & $5.06_{-056}^{+0.68}$ \\
\hline $292.6+01.2$ & NGC 3699 & 5336133687170599040 & 1.00 & 171.99097 & -59.95790 & 17.6 & 0.733 & 0.081 & $1.44_{-0.15}^{+0.19}$ \\
\hline $292.7+01.9$ & Wray $16-93$ & 5336505352135928448 & 1.00 & 172.70201 & -59.28498 & 18.4 & 0.438 & 0.129 & $4.92_{-2.10}^{+3.111}$ \\
\hline $292.8+01.1$ & He 2-67 & 5336037857882804736 & 0.69 & 172.19741 & -60.11048 & 17.6 & & & \\
\hline $292.9+01.0$ & PHR J1129-6012 & 5335989479389919104 & 0.35 & 172.45944 & -60.20221 & 17.2 & 0.345 & 0.063 & \\
\hline $293.1-00.0$ & BMP J1128-6121 & 5334404876960214656 & 0.95 & 172.05363 & -61.35083 & 18.9 & & & $\cdots$ \\
\hline $293.2-09.5$ & Fr 2-7 & 5231586379205084416 & 1.00 & 163.61411 & -70.21998 & 17.4 & 0.209 & 0.068 & \\
\hline $293.4+00.1$ & PHR J1130-6114 & 5334421541393316480 & 0.98 & 172.74558 & -61.24748 & 19.2 & 0.372 & 0.202 & \\
\hline $293.6+01.2$ & Hen $2-70$ & 5335879596943573888 & 0.98 & 173.79564 & -60.28361 & 15.7 & 0.110 & 0.038 & $4.74_{-0.52}^{+0.59}$ \\
\hline $293.6+10.9$ & BIDz 1 & 5369311141281715072 & 1.00 & 178.27780 & -50.84912 & 18.3 & 0.737 & 0.128 & $1.54_{-0.25}^{+0.34}$ \\
\hline $294.1+14.4$ & Lo 6 & 6142985384373962496 & 1.00 & 180.17948 & -47.55300 & 19.7 & 0.529 & 0.362 & $3.42_{-0.98}^{+1.02}$ \\
\hline $294.1+43.6$ & NGC 4361 & 3519614068578061568 & 1.00 & 186.12811 & -18.78492 & 13.1 & 0.965 & 0.044 & $1.01_{-0.04}^{+0.05}$ \\
\hline $294.2+01.3$ & PHR J1140-6022 & 5335831424542410368 & 0.81 & 175.15336 & -60.36937 & 20.9 & & & \\
\hline $294.6+04.7$ & NGC 3918 & 5342260406474812416 & 0.71 & 177.57403 & -57.18250 & 15.4 & 0.220 & 0.074 & $2.49_{-0.25}^{+0.28}$ \\
\hline $294.9-00.6$ & Hf 69 & 5333350548355207168 & 1.00 & 175.40533 & -62.48189 & 19.4 & 0.430 & 0.248 & $1.89_{-0.39}^{+0.494}$ \\
\hline $294.9-04.3$ & Hen 2-68 & 5237194683112374400 & 0.89 & 172.93936 & -65.97059 & 15.2 & 0.132 & 0.044 & $7.34_{-1.51}^{+1.88}$ \\
\hline $295.3-09.3$ & Hen 2-62 & 5228539671146215552 & 1.00 & 169.42977 & -70.82561 & 14.9 & 0.292 & 0.410 & $10.81_{-3.15}^{+3.15}$ \\
\hline $296.0-06.2$ & MPA J1137-6806 & 5234306472200493568 & 1.00 & 174.31494 & -68.11237 & 20.3 & -0.128 & 0.570 & $2.87_{-0.71}^{+0.34}$ \\
\hline $296.2-01.9$ & PaMo 1 & 5333115321569621760 & 0.50 & 177.43152 & -63.98079 & 20.9 & $\ldots$ & $\ldots$ & $\cdots$ \\
\hline $296.3-03.0$ & Hen 2-73 & 5332582092827700224 & 0.42 & 177.15873 & -65.14346 & 17.5 & & & \\
\hline $296.4-06.9$ & Hen $2-71$ & 5234243559525347968 & 1.00 & 174.79659 & -68.86915 & 13.8 & 0.076 & 0.062 & $7.45_{-1.48}^{+1.68}$ \\
\hline $296.6-20.0$ & NGC 3195 & 5198687896083997312 & 1.00 & 152.33683 & -80.85842 & 17.7 & 0.369 & 0.079 & $2.53_{-0.34}^{+0.48}$ \\
\hline $297.0+06.5$ & BMP J1209-5553 & 6075587378880414976 & 1.00 & 182.37135 & -55.89275 & 19.1 & -0.059 & 0.225 & $14.00_{-3.78}^{+3.85}$ \\
\hline $297.4+03.7$ & Hen $2-78$ & 6071280561769960576 & 0.99 & 182.29248 & -58.71035 & 15.5 & 0.038 & 0.028 & $14.56_{-315}^{+3.80}$ \\
\hline $298.3+06.6$ & Po 1 & 6072903883317795968 & 1.00 & 184.63642 & -55.90128 & 18.0 & 0.627 & 0.155 & \\
\hline $298.3-04.8$ & NGC 4071 & 5860341901722274688 & 1.00 & 181.06113 & -67.31013 & 18.5 & 0.376 & 0.153 & $2.22_{-038}^{+0.44}$ \\
\hline
\end{tabular}


Table A.1: continued.

\begin{tabular}{|c|c|c|c|c|c|c|c|c|c|}
\hline PN G & PN name & Gaia source ID & reliability & $\begin{array}{c}\text { RA } \\
(\mathrm{deg})\end{array}$ & $\begin{array}{c}\text { Dec } \\
(\mathrm{deg})\end{array}$ & $\begin{array}{c}\mathrm{G} \\
(\mathrm{mag})\end{array}$ & $\begin{array}{c}\omega \\
(\mathrm{mas})\end{array}$ & $\begin{array}{c}\sigma_{\omega} \\
\text { (mas) }\end{array}$ & $\begin{array}{c}\text { distance } \\
(\mathrm{kpc})\end{array}$ \\
\hline $298.7-07.5$ & PHR J1202-7001 & 5855902039382099968 & 0.21 & 180.73111 & -70.01583 & 19.9 & 0.322 & 0.384 & \\
\hline $299.0+18.4$ & K 1-23 & 6133278036730727296 & 1.00 & 187.71903 & -44.23781 & 18.7 & 0.325 & 0.217 & $3.01_{-0.69}^{+0.76}$ \\
\hline $299.1-08.4$ & MPA J1205-7101 & 5854994461239756800 & 1.00 & 181.41673 & -71.03245 & 17.5 & 0.077 & 0.079 & \\
\hline $299.2+01.0$ & PHR J1220-6134 & 6057925446869129600 & 0.38 & 185.03673 & -61.57128 & 20.9 & -0.892 & 1.278 & $9.10_{-2.75}^{+2.78}$ \\
\hline $299.2-04.1$ & PHR J1213-6643 & 5860474835237734144 & 0.84 & 183.39899 & -66.72021 & 18.7 & 0.444 & 0.152 & \\
\hline $299.5+02.4$ & Hen 2-82 & 6058295295126423936 & 0.89 & 185.97308 & -60.22027 & 20.9 & 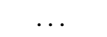 & . & $\ldots$ \\
\hline $299.7+00.1$ & PHR J1223-6236 & 6054613271184095232 & 0.21 & 185.98972 & -62.60614 & 21.1 & & & 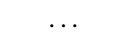 \\
\hline $299.7-01.5$ & MPA J1221-6413 & 6053490910387217920 & 0.97 & 185.46606 & -64.21819 & 20.7 & $\ldots$ & $\ldots$ & $\ldots$ \\
\hline $299.8-01.3$ & Hen 2-81 & 6053503382938739584 & 0.50 & 185.75581 & -64.02936 & 21.2 & & & \\
\hline $300.1+04.1$ & BMP J1229-5839 & 6059295438380078592 & 1.00 & 187.49004 & -58.65166 & 19.9 & 0.489 & 0.374 & \\
\hline $300.2+00.6$ & Hen 2-83 & 6054755761030163328 & 0.98 & 187.18321 & -62.09309 & 17.2 & 0.298 & 0.104 & $4.29_{-1.21}^{+1.64}$ \\
\hline $300.4-00.9$ & Hen 2-84 & 6053533482078258304 & 0.99 & 187.19519 & -63.74363 & 19.2 & 2.022 & 0.592 & $1.96_{-1.26}^{+1.69}$ \\
\hline $300.5-01.1$ & Hen 2-85 & 6053470775539684736 & 0.79 & 187.53185 & -63.88354 & 18.0 & 0.916 & 0.200 & $1.53_{-0.43}^{-0.26}$ \\
\hline $300.7-02.0$ & He $2-86$ & 5861205190154456192 & 1.00 & 187.62681 & -64.86825 & 17.4 & & & $\ldots$ \\
\hline $300.8-03.4$ & ESO 95-12 & 5860136258689477632 & 1.00 & 187.60217 & -66.24049 & 20.1 & -0.457 & 0.479 & \\
\hline $301.1-01.4$ & PM 1-68 & 5861374033935241088 & 0.94 & 188.65005 & -64.30470 & 18.6 & & & $\ldots$ \\
\hline $301.2-04.0$ & PHR J1234-6651 & 5859816060266111616 & 0.24 & 188.56938 & -66.86372 & 21.0 & & & $\ldots$ \\
\hline $301.3+09.0$ & MPA J1240-5347 & 6074692891815161728 & 1.00 & 190.18471 & -53.79069 & 19.5 & 0.330 & 0.343 & $\ldots$ \\
\hline $301.8+25.6$ & qparker-3 & 4040067691446079616 & 0.37 & 266.60532 & -37.21349 & 17.6 & 0.917 & 0.384 & $\ldots$ \\
\hline $301.9-02.1$ & MPA J1242-6459 & 5860308641513003136 & 0.73 & 190.60125 & -64.99017 & 19.8 & & & \\
\hline $302.0-01.6$ & MPA J1243-6428 & 5862586520319889920 & 0.85 & 190.83088 & -64.46704 & 18.4 & -0.587 & 0.297 & \\
\hline $302.1+00.3$ & RCW 69 & 6055200341668022400 & 1.00 & 191.11426 & -62.52191 & 19.4 & 0.607 & 0.268 & $1.32_{-0.23}^{+0.27}$ \\
\hline $302.1-05.3$ & Y-C 2-14 & 5856545180665018496 & 0.97 & 190.83902 & -68.21141 & 18.5 & 0.179 & 0.124 & $\ldots$ \\
\hline $302.2+02.5$ & Wray $16-120$ & 6056638537222985472 & 0.93 & 191.47734 & -60.33768 & 20.5 & & & \\
\hline $302.3-00.5$ & WRAY 17-56 & 5862901770945929472 & 0.91 & 191.61209 & -63.40775 & 16.8 & 0.270 & 0.056 & .. \\
\hline $303.7-01.9$ & Pre 22 & 5861683164163760896 & 0.74 & 194.71141 & -64.80956 & 20.2 & 0.283 & 0.540 & \\
\hline $304.2+05.9$ & Wray $16-122$ & 6060618082125746816 & 1.00 & 195.17175 & -56.89460 & 18.9 & -0.073 & 0.204 & $5.17_{-1.31}^{+1.46}$ \\
\hline $304.5-04.8$ & IC 4191 & 5857187746464509696 & 0.96 & 197.19711 & -67.64376 & 17.2 & $\ldots$ & 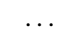 & $\ldots$ \\
\hline $304.7-02.1$ & PHR J1309-6457 & 5858960227897169024 & 0.36 & 197.25581 & -64.95659 & 21.0 & & & \\
\hline $304.8+05.1$ & Hen 2-88 & 6057322738402565632 & 1.00 & 196.45091 & -57.65680 & 14.8 & 0.143 & 0.026 & $6.05_{-0.88}^{+1.28}$ \\
\hline $304.8-01.4$ & MPA J1309-6415 & 5862040715937754240 & 0.78 & 197.37886 & -64.25899 & 21.1 & & & \\
\hline $305.1+17.1$ & ETHOS 3 & 170225876096 & 1.00 & 195.92000 & -45.62303 & 16.8 & 0.087 & 0.075 & $\ldots$ \\
\hline $305.3+03.0$ & Wray $17-58$ & 6055932925675151616 & 0.66 & 197.58203 & -59.75309 & 17.4 & 0.195 & 0.076 & \\
\hline $305.6-13.1$ & ESO 40-11 & 5790663302917359872 & 1.00 & 203.55954 & -75.77540 & 15.7 & 0.432 & 0.039 & $2.30_{-019}^{+0.23}$ \\
\hline $305.7-03.4$ & Wray $17-59$ & 5857924556722638208 & 1.00 & 199.87455 & -66.15226 & 15.9 & 0.211 & 0.033 & -0. \\
\hline $305.9+04.6$ & PHR J1314-5806 & 6063021270982325248 & 0.87 & 198.51805 & -58.10794 & 17.3 & 0.234 & 0.077 & \\
\hline $305.9-01.6$ & MPA J1319-6418 & 5859084610246081280 & 0.97 & 199.85016 & -64.31485 & 21.0 & & & \\
\hline $306.4-00.6$ & Th 2-A & 5865192156852977536 & 0.99 & 200.64144 & -63.35056 & 17.1 & 0.448 & 0.086 & $2.37_{-0.37}^{+0.48}$ \\
\hline $306.5-31.1$ & Fr $1-4$ & 6343834891409105664 & 1.00 & 335.46109 & -84.91065 & 16.0 & 0.639 & 0.043 & \\
\hline $306.7+06.6$ & PHR J1318-5601 & 6063529314064047616 & 0.33 & 199.57663 & -56.01955 & 20.5 & 1.756 & 0.636 & $2.16_{-0.96}^{+0.94}$ \\
\hline $306.7-01.5$ & MPA J1326-6407 & 5864899201285839616 & 0.38 & 201.63608 & -64.11972 & 20.1 & & & $\ldots$ \\
\hline $306.9-00.7$ & [GKF2010] MN12 & 5865160167931372672 & 0.90 & 201.82434 & -63.32441 & 19.5 & -0.810 & 0.465 & \\
\hline $307.0-02.0$ & MPA J1330-6438 & 5864092335920241536 & 0.40 & 202.54619 & -64.64092 & 18.2 & 0.595 & 0.241 & \\
\hline $307.2-03.4$ & NGC 5189 & 5863702868275424384 & 0.99 & 203.38685 & -65.97419 & 14.5 & 0.681 & 0.018 & $1.39_{-0.03}^{+0.04}$ \\
\hline $307.2-05.3$ & MPA J1337-6751 & 5850553155864892032 & 1.00 & 204.34326 & -67.85183 & 20.0 & 0.649 & 0.397 & $\ldots$ \\
\hline $307.2-09.0$ & Hen 2-97 & 5840002203581948160 & 1.00 & 206.34318 & -71.48226 & 13.9 & & & \\
\hline $307.3+02.0$ & PHR J1327-6032 & 5869500043300991232 & 0.75 & 201.80847 & -60.53524 & 20.8 & 1.395 & 1.202 & $1.62_{-049}^{+0.50}$ \\
\hline $307.3+02.0 \mathrm{a}$ & MPA J1327-6031 & 5869499631021431936 & 0.95 & 201.87690 & -60.53297 & 19.0 & & & $\ldots$ \\
\hline $307.3+05.0$ & Wray $16-128$ & 6062769826395293440 & 1.00 & 201.09135 & -57.52212 & 18.2 & 0.383 & 0.130 & \\
\hline $307.3-01.6$ & PHR J1332-6412 & 5864859623191472384 & 0.35 & 203.02318 & -64.20791 & 19.8 & 0.716 & 0.434 & \\
\hline $307.5-04.9$ & MyCn 18 & 5851342841411810304 & 1.00 & 204.89601 & -67.38105 & 14.2 & 0.247 & 0.079 & $3.21_{-0.48}^{+0.56}$ \\
\hline $307.7-03.1$ & PHR J1337-6535 & 5863831854666599424 & 1.00 & 204.46303 & -65.58841 & 19.8 & 0.415 & 0.350 & -0.48 \\
\hline $307.8-02.5$ & PKP1338-6456 & 5863912359619754624 & 0.97 & 204.60887 & -64.94512 & 18.7 & 0.143 & 0.169 & \\
\hline $308.2+03.5$ & PHR J1332-5851 & 5870222594291491840 & 0.44 & 203.05846 & -58.86088 & 19.6 & -1.648 & 0.346 & \\
\hline $308.2+07.7$ & MeWe 1-3 & 6064085976174124544 & 1.00 & 202.02048 & -54.69970 & 17.2 & 0.140 & 0.082 & $7.63_{-1.88}^{+2.11}$ \\
\hline $308.2-02.1$ & PHR J1341-6427 & 5863958161112598912 & 0.34 & 205.28141 & -64.46515 & 19.5 & -0.517 & 0.862 & \\
\hline $308.4+02.4$ & PHR J1335-5956 & 5869171117546506368 & 0.22 & 203.84780 & -59.94232 & 21.1 & & & \\
\hline $308.4-00.1$ & $\begin{array}{l}\text { 2MASS } \quad \text { J13395637- } \\
6230325\end{array}$ & 5865477578769818368 & 0.76 & 204.98485 & -62.50907 & 19.3 & 0.133 & 0.254 & \\
\hline $308.5+02.5$ & PHR J1336-5953 & 5869170013686928000 & 1.00 & 204.09599 & -59.89011 & 19.0 & 0.209 & 0.198 & \\
\hline $308.6-12.2$ & Hen 2-105 & 5796437220731788288 & 1.00 & 213.85311 & -74.21281 & 14.6 & 0.271 & 0.032 & $3.36_{-0.30}^{+0.35}$ \\
\hline $309.0+00.8$ & Hen 2-96 & 5865873853960686208 & 0.99 & 205.65063 & -61.37468 & 17.5 & 3.314 & 0.695 & $0.36_{-0.09}^{+0.39}$ \\
\hline $309.0-04.2$ & Hen 2-99 & 5851865148069389568 & 1.00 & 208.12778 & -66.39072 & 13.2 & 0.207 & 0.013 & $4.03_{-0.19}^{+0.21}$ \\
\hline $309.1-04.3$ & NGC 5315 & 5851109332606802432 & 0.98 & 208.48733 & -66.51414 & 13.1 & 1.037 & 0.200 & $1.17_{-0.24}^{+0.39}$ \\
\hline $309.2+01.3$ & VBRC 5 & 5868906135192925696 & 0.45 & 205.99924 & -60.82895 & 20.8 & 0.744 & 1.122 & \\
\hline $309.5-02.9$ & $\mathrm{MaC} 1-2$ & 5852194829861430272 & 0.85 & 208.61411 & -64.99230 & 19.1 & & & \\
\hline $309.6-04.8$ & MPA J1400-6647 & 5850867753619609216 & 1.00 & 210.15443 & -66.79952 & 20.6 & 0.353 & 0.765 & $4.56_{-1.30}^{+1.31}$ \\
\hline $309.8-01.6$ & MPA J1354-6337 & 5864421983242008832 & 0.96 & 208.59329 & -63.62163 & 20.4 & -0.017 & 0.817 & $\ldots$ \\
\hline $310.1-03.7$ & PHR J1402-6535 & 5851256431021374208 & 0.92 & 210.54134 & -65.59258 & 19.5 & 0.343 & 0.303 & \\
\hline $310.3+24.7$ & Lo 8 & 6162542191540458112 & 1.00 & 201.40622 & -37.60408 & 12.9 & 0.670 & 0.061 & $1.47_{-0.12}^{+0.15}$ \\
\hline $310.6+01.4$ & WKG 3 & 5867401487901832832 & 1.00 & 208.60258 & -60.45619 & 18.2 & 0.517 & 0.113 & \\
\hline
\end{tabular}


Table A.1: continued.

\begin{tabular}{|c|c|c|c|c|c|c|c|c|c|}
\hline PN G & PN name & Gaia source ID & reliability & $\begin{array}{c}\text { RA } \\
(\mathrm{deg})\end{array}$ & $\begin{array}{c}\text { Dec } \\
(\mathrm{deg})\end{array}$ & $\begin{array}{c}\mathrm{G} \\
(\mathrm{mag})\end{array}$ & $\begin{array}{c}\omega \\
(\mathrm{mas})\end{array}$ & $\begin{array}{c}\sigma_{\omega} \\
\text { (mas) }\end{array}$ & $\begin{array}{c}\text { distance } \\
(\mathrm{kpc})\end{array}$ \\
\hline $310.7-02.9$ & Hen 2-103 & 5852071439644421504 & 0.49 & 211.40322 & -64.68368 & 20.5 & -0.356 & 1.100 & $3.46_{-10}^{+1.05}$ \\
\hline $310.8-05.9$ & $\operatorname{LoTr} 7$ & 5847795443251442304 & 1.00 & 213.85047 & -67.53176 & 19.4 & -0.154 & 0.252 & \\
\hline $311.0+02.4$ & SuWt 2 & 5870592987893097984 & 0.99 & 208.93010 & -59.37773 & 11.9 & 0.443 & 0.015 & $2.09^{+0.07}$ \\
\hline $311.1-06.0$ & WRAY $16-150$ & 5847622201453884672 & 0.90 & 214.78111 & -67.53673 & 17.2 & 0.061 & 0.079 & \\
\hline $311.4+02.8$ & Hen 2-102 & 5870632570311652608 & 1.00 & 209.55786 & -58.90879 & 17.1 & 0.121 & 0.076 & $5.10^{+1.00}$ \\
\hline $311.5-02.7$ & WRAY 16-146 & 5853602028546852352 & 0.79 & 212.94274 & -64.27333 & 15.0 & 0.058 & 0.114 & \\
\hline $311.7+07.3$ & PHR J1351-5429 & 6064797940000480512 & 1.00 & 207.94352 & -54.49483 & 17.7 & 0.257 & 0.122 & $6.69^{+2.19}$ \\
\hline $312.0+04.1$ & PHR J1359-5728 & 5871340243484051456 & 0.96 & 209.91576 & -57.48050 & 18.8 & 0.252 & 0.195 & \\
\hline $312.3+05.7$ & BMP J1358-5552 & 5872363137226865536 & 0.90 & 209.69027 & -55.87967 & 18.4 & 0.310 & 0.214 & \\
\hline $312.3+10.5$ & NGC 5307 & 6093370471935953152 & 1.00 & 207.76350 & -51.20581 & 14.7 & 0.288 & 0.056 & $3.19_{-043}^{+0.53}$ \\
\hline $312.6-01.8$ & Hen 2-107 & 5854138766383247232 & 0.65 & 214.68051 & -63.11948 & 14.6 & 0.259 & 0.021 & $3.43_{-023}^{+0.27}$ \\
\hline $312.7+09.5$ & $\mathrm{~Pa} 42$ & 6090071387288211968 & 0.52 & 208.70704 & -52.08019 & 20.8 & & & \\
\hline $313.0+03.2$ & MPA J1408-5804 & 5868135858621005440 & 0.96 & 212.20597 & -58.07353 & 19.2 & -0.281 & 0.270 & $\ldots$ \\
\hline $313.1+02.1$ & PM 1-79 & 5867815316585092608 & 0.81 & 214.21576 & -58.88612 & 19.6 & $\ldots$ & $\ldots$ & $\ldots$ \\
\hline $313.3+00.3$ & CGR 1 & 5866663749945999360 & 0.29 & 214.61441 & -60.78638 & 21.1 & & & $\ldots$ \\
\hline $313.3+01.1$ & WRAY 17-66 & 5866938490411910528 & 0.67 & 213.97198 & -60.02715 & 20.5 & & & 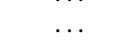 \\
\hline $313.4+06.2$ & MPA J1405-5507 & 5896420348552980608 & 1.00 & 211.38445 & -55.12918 & 16.5 & 0.031 & 0.068 & . \\
\hline $313.7-37.1$ & $\mathrm{~Pa} 162$ & 6357229863612184448 & 1.00 & 333.06473 & -76.43430 & 15.1 & 0.292 & 0.032 & \\
\hline $313.8+10.3$ & Fr $2-8$ & 6090256513264898816 & 1.00 & 210.17371 & -51.04106 & 16.1 & 0.461 & 0.045 & $2.08^{+0.21}$ \\
\hline $313.8-01.7$ & MPA J1428-6234 & 5853510704716141184 & 0.30 & 217.21192 & -62.57044 & 19.5 & 0.145 & 0.337 & \\
\hline $313.8-05.7$ & BMP J1442-6615 & 5848879424277154176 & 0.95 & 220.69393 & -66.25074 & 20.3 & -0.565 & 0.628 & $5.01_{-129}^{+1.32}$ \\
\hline $313.8-12.6$ & LoTr 11 & 5795257990207545984 & 1.00 & 230.29353 & -72.22385 & 18.3 & 0.665 & 0.116 & $1.91_{-037}^{-0.55}$ \\
\hline $313.9+02.8$ & PHR J1416-5809 & 5891918673080594176 & 0.99 & 214.15699 & -58.15893 & 16.2 & 0.171 & 0.047 & \\
\hline $314.0+10.6$ & MeWe 2-4 & 6090271154808162048 & 1.00 & 210.31417 & -50.66927 & 17.9 & 0.848 & 0.109 & $1.25_{-0.15}^{+0.18}$ \\
\hline $314.4+02.2$ & PM 1-81 & 5891103385192215296 & 0.75 & 215.33286 & -58.63937 & 16.2 & 0.701 & 0.045 & \\
\hline $315.0-00.3$ & Hen 2-111 & 5878442989838460416 & 0.94 & 218.32696 & -60.82677 & 16.5 & 0.358 & 0.054 & $2.53_{-028}^{+0.33}$ \\
\hline $315.1-13.0$ & Hen 2-131 & 5794858077215766656 & 1.00 & 234.29665 & -71.91471 & 10.9 & 0.368 & 0.030 & $2.56_{-0.18}^{+0.28}$ \\
\hline $315.4-08.4$ & PHR J1510-6754 & 5800264758167606144 & 1.00 & 227.59173 & -67.90670 & 15.5 & 0.888 & 0.026 & $1.08^{+0.03}$ \\
\hline $315.7+02.1$ & St 58 & 5890988417518712064 & 0.82 & 217.68456 & -58.26072 & 19.2 & 0.682 & 0.294 & \\
\hline $315.7+05.5$ & LoTr 8 & 5895881038094691200 & 0.98 & 215.49969 & -55.03803 & 18.1 & 0.687 & 0.214 & $3.95_{-1.95}^{+2.53}$ \\
\hline $315.7+42.0$ & EC $13290-1933$ & 6292958495525136768 & 1.00 & 202.94284 & -19.80717 & 14.4 & 0.803 & 0.040 & \\
\hline $315.7-04.2$ & Wray $16-158$ & 5873884758224326144 & 0.79 & 223.15061 & -64.03949 & 19.8 & & & \\
\hline $315.9+08.2$ & MeWe 1-4 & 5897337302564816384 & 0.33 & 214.37685 & -52.43920 & 18.6 & 1.010 & 0.277 & $1.60_{-0.47}^{+0.63}$ \\
\hline $316.0+02.9$ & MPA J1430-5721 & 5891638606833232640 & 0.78 & 217.60431 & -57.36280 & 19.8 & 0.435 & 0.418 & \\
\hline $316.1+08.4$ & Hen 2-108 & 5897352631316651264 & 1.00 & 214.53700 & -52.17764 & 12.7 & 0.239 & 0.022 & $3.64_{-0.26}^{+0.31}$ \\
\hline $316.2+00.8$ & GLMP 387 & 5879195502480164864 & 0.96 & 219.58332 & -59.19617 & 19.8 & & & \\
\hline $316.3+08.8$ & PHR J1418-5144 & 5897438771177986432 & 1.00 & 214.60745 & -51.74406 & 17.2 & 0.857 & 0.081 & $1.19_{-010}^{+0.12}$ \\
\hline $316.7+01.6$ & MPA J1439-5816 & 5879307068557653120 & 0.90 & 219.85434 & -58.26712 & 21.1 & & & \\
\hline $316.7-05.8$ & MPA J1508-6455 & 5872884511895152768 & 1.00 & 227.02695 & -64.93023 & 13.5 & 0.216 & 0.017 & $4.01_{-0.26}^{+0.30}$ \\
\hline $316.9+01.8$ & MPA J1440-5802 & 5879404203516670592 & 0.78 & 220.11401 & -58.03873 & 20.6 & 1.222 & 1.388 & \\
\hline $316.9+03.7$ & Pa 61 & 5891821366296551808 & 0.97 & 218.64216 & -56.30914 & 20.2 & 0.006 & 0.570 & $\ldots$ \\
\hline $316.9+04.1$ & PHR J1433-5557 & 5892592020849979136 & 0.99 & 218.36112 & -55.95531 & 18.1 & 0.342 & 0.123 & \\
\hline $317.1-05.7$ & NGC 5844 & 5873264285765311232 & 1.00 & 227.66986 & -64.67387 & 17.9 & 0.716 & 0.105 & $1.44^{+0.22}$ \\
\hline $317.2+08.6$ & PHR J1424-5138 & 5898716231900175616 & 0.96 & 216.13476 & -51.64421 & 13.0 & 0.403 & 0.036 & $2.34_{-0.18}^{+0.18}$ \\
\hline $317.3-03.8$ & PHR J1503-6257 & 5873669043473542016 & 0.41 & 225.99495 & -62.96201 & 19.1 & 0.796 & 0.231 & -0. \\
\hline $318.2-02.3$ & PHR J1504-6113 & 5874664140219303808 & 0.61 & 226.16528 & -61.21960 & 20.1 & -0.274 & 0.514 & \\
\hline $318.3-02.0$ & Hen 2-114 & 5876361167674415104 & 1.00 & 226.03588 & -60.88894 & 20.6 & 2.337 & 1.386 & $3.24_{-1.24}^{+1.21}$ \\
\hline $318.3-02.5$ & Hen 2-116 & 5876147965487613056 & 1.00 & 226.50780 & -61.35571 & 19.9 & 0.440 & 0.416 & $2.48_{-0.65}^{+0.69}$ \\
\hline $318.4+41.4$ & Abell 36 & 6292074655679874688 & 1.00 & 205.17236 & -19.88201 & 11.5 & 2.391 & 0.061 & $0.41_{-0.01}^{-0.65}$ \\
\hline $318.4-03.0$ & ESO 135-04 & 5876077734143104768 & 0.98 & 227.18150 & -61.73699 & 20.7 & -0.596 & 1.482 & $\ldots$ \\
\hline $318.5-02.0$ & WRAY 16-161 & 5876367043212127616 & 0.79 & 226.38496 & -60.81421 & 19.7 & & & \\
\hline $318.5-02.3$ & Pre 37 & 5876172841955547392 & 0.92 & 226.57760 & -61.07269 & 20.5 & 1.968 & 1.067 & $\ldots$ \\
\hline $319.0-02.7$ & MPA J1511-6108 & 5876133465670724864 & 0.98 & 227.83731 & -61.14797 & 18.1 & 0.303 & 0.157 & $\ldots$ \\
\hline $319.1+02.1$ & PHR1454-5648 & 5881036394197872384 & 0.99 & 223.53883 & -56.80473 & 11.3 & 0.735 & 0.025 & $\ldots$ \\
\hline $319.2+06.8$ & Hen $2-112$ & 5895030978161528192 & 0.96 & 220.12888 & -52.58248 & 18.3 & $\ldots$ & $\ldots$ & . \\
\hline $319.3-02.6$ & BMP J1513-6052 & 5876220911242751488 & 0.44 & 228.40182 & -60.86768 & 21.1 & & $\ldots$ & $\ldots$ \\
\hline $319.5-01.1$ & PrePa 3 & 5876600410193992960 & 0.97 & 227.23505 & -59.55450 & 20.9 & & & \\
\hline $319.6+15.7$ & IC 4406 & 6102472263541814272 & 1.00 & 215.60891 & -44.15061 & 17.2 & 0.876 & 0.117 & $1.24_{-0.16}^{+0.21}$ \\
\hline $320.0+03.9$ & PHR J1453-5448 & 5893638201857294464 & 0.96 & 223.42114 & -54.80256 & 19.1 & 0.012 & 0.273 & $\cdots$ \\
\hline $320.1-02.7$ & PHR J1519-6032 & 5875878722577809280 & 0.37 & 229.85483 & -60.54419 & 21.0 & & & \\
\hline $320.1-09.6$ & Hen $2-138$ & 5822458022416453120 & 1.00 & 239.00700 & -66.15259 & 10.9 & 0.203 & 0.026 & $4.41_{-0.42}^{+0.50}$ \\
\hline $320.3-28.8$ & Hen 2-434 & 6367469439307095936 & 1.00 & 293.45563 & -74.54963 & 14.1 & 0.143 & 0.040 & $6.26_{-1.10}^{+0.40}$ \\
\hline $320.6-04.8$ & PHR J1532- & 5827410630783549312 & 0.96 & 233.24741 & -62.05096 & 16.6 & 0.062 & 0.051 & $10.52_{-2.29}^{+2.57}$ \\
\hline $320.7+04.2$ & BMP J1457-5413 & 5893724513518808832 & 0.98 & 224.25950 & -54.23281 & 18.7 & 0.564 & 0.212 & $\ldots$ \\
\hline $320.7-11.4$ & $\mathrm{Kn} 72$ & 5821376785848327552 & 1.00 & 243.30298 & -67.08312 & 17.6 & 0.100 & 0.077 & \\
\hline $320.8+01.6$ & $\mathrm{~Pa} 65$ & 5880534364018604672 & 0.89 & 226.63646 & -56.43468 & 20.8 & & & \\
\hline $320.9+00.0$ & IRAS $15093-5732$ & 5880168742050611456 & 0.44 & 228.30424 & -57.72442 & 19.9 & -0.872 & 0.823 & \\
\hline $320.9+02.0$ & Hen $2-117$ & 5881321133340445696 & 1.00 & 226.49663 & -55.98795 & 17.8 & 0.794 & 0.205 & $1.71^{+0.73}$ \\
\hline $321.0+01.4$ & Pa 179 & 5880493815227788160 & 0.68 & 227.12938 & -56.55035 & 19.5 & 0.010 & 0.300 & \\
\hline $321.0+03.9$ & Hen 2-113 & 5899715786733345536 & 0.70 & 224.97280 & -54.30209 & 11.6 & 0.482 & 0.027 & $1.97_{-010}^{+0.11}$ \\
\hline
\end{tabular}


Table A.1: continued.

\begin{tabular}{|c|c|c|c|c|c|c|c|c|c|}
\hline PN G & PN name & Gaia source ID & reliability & $\begin{array}{c}\text { RA } \\
\text { (deg) }\end{array}$ & $\begin{array}{c}\text { Dec } \\
(\mathrm{deg})\end{array}$ & $\begin{array}{c}\mathrm{G} \\
(\mathrm{mag})\end{array}$ & $\begin{array}{c}\omega \\
(\mathrm{mas})\end{array}$ & $\begin{array}{c}\sigma_{\omega} \\
(\mathrm{mas})\end{array}$ & $\begin{array}{c}\text { distance } \\
(\mathrm{kpc})\end{array}$ \\
\hline $321.0+08.3$ & MeWe 1-5 & 5904325489235726464 & 1.00 & 221.64627 & -50.39020 & 19.4 & & & \\
\hline $321.0-00.6$ & [GKF2010] MN18 & 5877088151005347072 & 0.96 & 229.17079 & -58.37389 & 12.2 & 0.171 & 0.019 & $\ldots$ \\
\hline $321.0-03.8$ & HaTr 2 & 5875572813518489856 & 1.00 & 232.57741 & -61.02759 & 18.3 & 0.061 & 0.148 & $\ldots$ \\
\hline $321.2-01.9$ & MPA J1522-5917 & 5876787705124856320 & 0.92 & 230.74254 & -59.29567 & 19.7 & 0.339 & 0.433 & \\
\hline $321.3+02.8$ & Hen 2-115 & 5887420772835970944 & 0.93 & 226.31996 & -55.18625 & 16.7 & -0.395 & 0.198 & $5.94_{-120}^{+1.28}$ \\
\hline $321.3-16.7$ & He 2-185 & 5808014459690304000 & 1.00 & 255.32179 & -70.10091 & 16.0 & -0.472 & 0.386 & $\ldots$ \\
\hline $321.4-05.1$ & MPA J1539-6147 & 5827334493954740992 & 0.99 & 234.96814 & -61.78960 & 17.1 & 0.114 & 0.064 & \\
\hline $321.6+02.2$ & CVMP 1 & 5886591161255356416 & 1.00 & 227.35484 & -55.55159 & 20.2 & 0.145 & 0.655 & $1.41^{+0.35}$ \\
\hline $321.8+01.9$ & Hen $2-120$ & 5886595490588171392 & 1.00 & 227.98431 & -55.66298 & 20.2 & 0.071 & 0.625 & $2.42^{+0.744}$ \\
\hline $322.1-06.6$ & Мy 91 & 5832448803513249792 & 0.96 & 238.04457 & -62.51297 & 19.0 & $\ldots$ & $\ldots$ & $\ldots$ \\
\hline $322.2-00.4$ & BMP J1522-5729 & 5883150961168950144 & 0.59 & 230.74519 & -57.49984 & 21.7 & $\ldots$ & . & $\ldots$ \\
\hline $322.4-00.1$ & Pe $2-8$ & 5883255655307867008 & 0.98 & 230.92868 & -57.15701 & 17.8 & & & \\
\hline $322.4-02.6$ & Mz 1 & 5881838006914886784 & 1.00 & 233.56927 & -59.15228 & 18.1 & 0.794 & 0.128 & $1.41_{-0.29}^{+0.29}$ \\
\hline $322.5-05.2$ & NGC 5979 & 5833343531156787840 & 1.00 & 236.92156 & -61.21822 & 16.3 & 0.263 & 0.051 & $3.46_{-0.44}^{+0.52}$ \\
\hline $322.6-04.2$ & PM 1-101 & 5833618615230285440 & 0.99 & 235.78017 & -60.42256 & 18.6 & & & $\ldots$ \\
\hline $322.7-01.4$ & MPA J1530-5801 & 5882224618322318208 & 0.96 & 232.71210 & -58.02467 & 19.8 & 0.241 & 0.473 & \\
\hline $323.1-02.5$ & Hen 2-132 & 5881952420494531328 & 1.00 & 234.50510 & -58.74515 & 16.9 & 0.269 & 0.065 & $3.53^{+0.74}$ \\
\hline $323.6-04.5$ & KFW 11 & 5833546803381125632 & 1.00 & 237.62063 & -59.98047 & 14.9 & -0.162 & 0.134 & \\
\hline $323.7-04.8$ & PHR J1552-6009 & 5833868479190000768 & 0.86 & 238.09743 & -60.15500 & 18.5 & 0.222 & 0.157 & $\ldots$ \\
\hline $323.9+00.2$ & GLIPN J1530-5557 & 5883716385032254720 & 0.90 & 232.67322 & -55.95766 & 17.8 & 0.264 & 0.140 & \\
\hline $323.9+02.4$ & Hen 2-123 & 5887196472462012928 & 0.99 & 230.58071 & -54.13687 & 16.7 & 0.178 & 0.073 & $4.24_{-069}^{+0.80}$ \\
\hline $324.0+03.5$ & PM 1-89 & 5888049732191403904 & 0.43 & 229.78650 & -53.16382 & 14.8 & 0.299 & 0.024 & \\
\hline $324.0-01.6$ & PHR J1539-5727 & 5882594058567764224 & 0.99 & 234.89496 & -57.45063 & 16.3 & 0.353 & 0.048 & \\
\hline $324.1+09.0$ & ESO 223-10 & 5903448633025639936 & 1.00 & 225.41955 & -48.35078 & 16.7 & 0.263 & 0.072 & $5.85_{-1.84}^{+2.90}$ \\
\hline $324.2+02.5$ & Hen 2-125 & 5887229462174068608 & 0.83 & 230.90140 & -53.85783 & 15.9 & 0.114 & 0.045 & $7.11_{-1.37}^{+1.66}$ \\
\hline $324.3-11.9$ & Kn 73 & 5816227639772060032 & 1.00 & 249.88137 & -64.80274 & 16.4 & 0.096 & 0.054 & -1 \\
\hline $324.4+03.8$ & PHR J1520- & 5888086084760690432 & 0.69 & 230.11440 & -52.72341 & 20.8 & & & \\
\hline $324.8-01.1$ & Hen 2-133 & 5882747955832834944 & 1.00 & 235.49503 & -56.60714 & 18.9 & 1.369 & 0.605 & \\
\hline $325.0+03.2$ & Hen 2-129 & 5888795544644516608 & 0.94 & 231.38603 & -52.84388 & 17.6 & -0.478 & 0.160 & $8.69^{+1.92}$ \\
\hline $325.4-04.0$ & Hen 2-141 & 5835567460898896128 & 0.99 & 239.78649 & -58.39769 & 18.1 & 0.174 & 0.137 & $4.35_{-0.95}^{+1.68}$ \\
\hline $325.7+02.2$ & BMP J1533 & 5888601549601893632 & 0.72 & 233.33609 & -53.31693 & 16.8 & 0.128 & 0.064 & \\
\hline $325.8+04.5$ & Hen 2-128 & 5889777649042432256 & 0.99 & 231.28264 & -51.32838 & 15.8 & -0.692 & 0.165 & $\ldots$ \\
\hline $325.8-05.4$ & BMP J1608-5913 & 5834543476289552768 & 1.00 & 242.03954 & -59.22859 & 18.7 & 0.077 & 0.189 & \\
\hline $325.8-12.8$ & Hen 2-182 & 5817579661117922688 & 1.00 & 253.64639 & -64.24129 & 13.1 & -0.120 & 0.056 & $9.15_{-1.33}^{+1.44}$ \\
\hline $325.9+09.4$ & BMP J1509-4707 & 5903941763984739072 & 1.00 & 227.43509 & -47.11880 & 15.8 & 0.113 & 0.044 & \\
\hline $326.0-02.4$ & FP J1554-5651 & 5836034414070315136 & 0.96 & 238.71239 & -56.86606 & 20.3 & -0.263 & 0.805 & $3.15_{-0.82}^{+0.84}$ \\
\hline $326.0-06.5$ & Hen 2-151 & 5831451649598626560 & 1.00 & 243.92618 & -59.90029 & 12.7 & 0.318 & 0.136 & $10.94_{-425}^{+4.10}$ \\
\hline $326.1-01.9$ & vBe 3 & 5836064513277457664 & 0.96 & 238.24591 & -56.40724 & 20.1 & 0.461 & 0.617 & $7.99_{-241}^{+2.41}$ \\
\hline $326.1-03.3$ & WRAY 16-197 & 5835757577647289856 & 0.98 & 239.94778 & -57.50159 & 17.4 & -0.709 & 0.263 & \\
\hline $326.4+01.5$ & PHR J1539-5325 & 5885927846499087360 & 0.25 & 234.95531 & -53.42356 & 20.1 & 0.694 & 0.697 & \\
\hline $326.4+07.0$ & $\mathrm{NeVe} 3-2$ & 5902564934924665984 & 1.00 & 229.93283 & -48.99859 & 15.3 & 0.245 & 0.030 & $3.55_{-0.33}^{+0.40}$ \\
\hline $326.5-03.4$ & PHR J1602-5714 & 5835747059266833664 & 0.43 & 240.61516 & -57.23538 & 20.4 & -2.828 & 0.834 & $\ldots$ \\
\hline $326.6+05.7$ & BMP J1525-4957 & 5890463813006531840 & 0.96 & 231.30861 & -49.96119 & 19.9 & -0.102 & 0.527 & \\
\hline $326.6+42.2$ & IC 972 & 6291509197468092672 & 0.54 & 211.10797 & -17.22794 & 17.3 & 0.449 & 0.105 & $2.79_{-0.64}^{+0.93}$ \\
\hline $326.9+02.2$ & PM 1-97 & 5886032300143619328 & 0.26 & 234.92930 & -52.60939 & 17.0 & 0.189 & 0.064 & $\ldots$ \\
\hline $327.1-01.8$ & He $2-140$ & 5836494250511506432 & 0.85 & 239.53371 & -55.69735 & 16.1 & 0.083 & 0.054 & \\
\hline $327.1-02.2$ & Hen 2-142 & 5836438239720535040 & 0.98 & 239.99012 & -55.92585 & 14.8 & 0.273 & 0.040 & $3.41_{-0.41}^{+0.53}$ \\
\hline $327.1-09.2$ & PHR J1637-6059 & 5830575338803704448 & 0.79 & 249.42643 & -60.98411 & 17.9 & 0.169 & 0.105 & $\ldots$ \\
\hline $327.2-02.5$ & MPA J1601-5608 & 5836233490046582912 & 0.90 & 240.44834 & -56.14047 & 17.8 & -0.042 & 0.110 & $\ldots$ \\
\hline $327.3-02.5$ & MPA J1602-5603a & 5836239919701660672 & 0.67 & 240.52982 & -56.06174 & 20.8 & -2.596 & 2.321 & 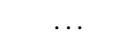 \\
\hline $327.5+13.3$ & Hen $2-118$ & 6004438325045425664 & 1.00 & 226.55714 & -42.99903 & 14.5 & & & \\
\hline $327.5-02.2$ & MPA J1602-5543 & 5836526376829511808 & 0.96 & 240.54660 & -55.72516 & 15.9 & 0.191 & 0.038 & \\
\hline $327.7-05.4$ & KoRe 1 & 5835120578152346240 & 1.00 & 244.79327 & -57.98203 & 18.8 & 0.444 & 0.194 & $14.77_{-530}^{+5.10}$ \\
\hline $327.8+10.0$ & NGC 5882 & 6000019147299399936 & 1.00 & 229.20812 & -45.64963 & 13.3 & 0.462 & 0.030 & $1.99_{-011}^{+0.12}$ \\
\hline $327.8-01.6$ & He 2-143 & 5836606709883180160 & 0.96 & 240.24649 & -55.09438 & 18.2 & & & \\
\hline $327.8-06.1$ & Hen 2-158 & 5832053288628607488 & 0.99 & 245.87767 & -58.32302 & 14.9 & -0.007 & 0.056 & $15.14_{-3.44}^{+3.70}$ \\
\hline $327.8-07.2$ & Hen 2-163 & 5831765457042662528 & 0.94 & 247.37994 & -59.15705 & 20.2 & 0.554 & 0.609 & $4.74_{-1.58}^{+1.60}$ \\
\hline $328.0-01.6$ & PM 1-105 & 5836638797516192256 & 0.49 & 240.46188 & -54.89448 & 18.6 & 0.084 & 0.467 & $\ldots$ \\
\hline $328.2+01.3$ & Lo 10 & 5981924591832069888 & 0.98 & 237.37094 & -52.50487 & 20.1 & 0.122 & 0.604 & \\
\hline $328.2+14.3$ & Mu 1 & 6004659807917992320 & 1.00 & 226.57130 & -41.75515 & 18.1 & 0.196 & 0.155 & $4.50_{-1.02}^{+1.12}$ \\
\hline $328.2-03.9$ & BMP J1613-5633 & 5835460572057022336 & 0.53 & 243.46524 & -56.55452 & 20.2 & $\ldots$ & & \\
\hline $328.4-02.8$ & PM 1-106 & 5932373141911732352 & 0.70 & 242.33415 & -55.60285 & 18.9 & & & \\
\hline $328.5+06.0$ & PHR J1533-4834 & 5986526735217715456 & 1.00 & 233.39162 & -48.57347 & 18.7 & 0.822 & 0.244 & $2.60_{-095}^{+1.14}$ \\
\hline $328.5+06.2$ & PHR J1533-4824 & 5986532468951904128 & 1.00 & 233.27861 & -48.41239 & 19.3 & 0.468 & 0.321 & $2.68_{-0.71}^{+0.77}$ \\
\hline $328.7-04.6$ & MPA J1619-5638 & 5931322799003585024 & 1.00 & 244.97872 & -56.63736 & 20.5 & 0.184 & 0.872 & \\
\hline $328.8+13.5$ & $\mathrm{~Pa} 33$ & 6003852732024802816 & 1.00 & 227.80480 & -42.17312 & 18.1 & 0.708 & 0.145 & $2.14_{-057}^{+1.01}$ \\
\hline $329.0+01.9$ & Sp 1 & 5982072132545824128 & 1.00 & 237.92059 & -51.52461 & 13.7 & 0.718 & 0.017 & $1.32_{-0.03}^{+0.03}$ \\
\hline $329.1-01.4$ & MPA J1606-5407 & 5932794976387644288 & 0.99 & 241.66145 & -54.12251 & 19.9 & & & \\
\hline $329.3-02.8$ & $\mathrm{Mz} 2$ & 5931763045992356736 & 0.98 & 243.63499 & -54.95130 & 16.8 & 0.109 & 0.075 & $3.24_{-0.39}^{+0.43}$ \\
\hline $329.4-02.7$ & Hen 2-149 & 5931790744348033792 & 0.90 & 243.60070 & -54.79413 & 18.3 & 0.304 & 0.691 & \\
\hline
\end{tabular}


Table A.1: continued.

\begin{tabular}{|c|c|c|c|c|c|c|c|c|c|}
\hline PN G & PN name & Gaia source ID & reliability & $\begin{array}{c}\text { RA } \\
(\mathrm{deg})\end{array}$ & $\begin{array}{c}\text { Dec } \\
(\mathrm{deg})\end{array}$ & $\begin{array}{c}\mathrm{G} \\
(\mathrm{mag})\end{array}$ & $\begin{array}{c}\omega \\
(\mathrm{mas})\end{array}$ & $\begin{array}{c}\sigma_{\omega} \\
\text { (mas) }\end{array}$ & $\begin{array}{c}\text { distance } \\
(\mathrm{kpc})\end{array}$ \\
\hline $329.5+01.7$ & VBRC 7 & 5982091545782770944 & 0.67 & 238.71184 & -51.37643 & 19.3 & 0.820 & 0.365 & $1.53_{-038}^{+0.44}$ \\
\hline $329.5-02.2$ & $\mathrm{HeFa} 1$ & 5932562670265887616 & 0.96 & 243.14107 & -54.39455 & 15.3 & 0.311 & 0.039 & $3.00_{-0.32}^{+0.38}$ \\
\hline $329.6-02.7$ & PHR J1615-5437 & 5931796379368641792 & 0.85 & 243.83473 & -54.62317 & 17.8 & 0.092 & 0.144 & \\
\hline $329.7+06.9$ & PHR J1536-4711 & 5987071543201574784 & 0.99 & 234.01556 & -47.19256 & 20.8 & & & \\
\hline $329.8-02.1$ & BMP J1613-5406 & 5932573046823712256 & 1.00 & 243.25850 & -54.10898 & 20.0 & 1.942 & 0.894 & $1.53^{+0.52}$ \\
\hline $329.9+03.7$ & BMP J1548-4936 & 5982837598830962048 & 0.93 & 237.21726 & -49.61340 & 19.6 & 0.095 & 0.479 & \\
\hline $330.1+02.6$ & MPA J1554-5022 & 5982575983753257344 & 0.90 & 238.53719 & -50.37765 & 18.9 & -0.500 & 0.314 & $\ldots$ \\
\hline $330.2+04.2$ & PHR J1548-4903 & 5985891251822728320 & 0.53 & 237.11030 & -49.05922 & 20.8 & & & \\
\hline $330.2+05.9$ & Lo 9 & 5986249726990491520 & 0.82 & 235.55424 & -47.67923 & 20.4 & 1.255 & 0.730 & $\ldots$ \\
\hline $330.5-01.4$ & IRAS $16097-5257$ & 5933051471867770240 & 0.99 & 243.42349 & -53.08092 & 21.1 & & & \\
\hline $330.6-02.1$ & Hen 2-153 & 5933070468426290944 & 0.62 & 244.30984 & -53.53489 & 20.2 & 0.973 & 0.957 & $4.27_{-1.67}^{+1.68}$ \\
\hline $330.6-03.6$ & Hen 2-159 & 5931925601916493312 & 0.97 & 246.08906 & -54.60090 & 16.1 & 0.157 & 0.206 & $4.84_{-1.23}^{+1.81}$ \\
\hline $330.9+04.3$ & Wray 16-189 & 5983021831411466240 & 1.00 & 237.83272 & -48.43544 & 18.5 & 0.444 & 0.189 & $4.12_{-1.47}^{+1.97}$ \\
\hline $331.0-02.7$ & Hen $2-157$ & 5932279305469535360 & 0.92 & 245.55967 & -53.68173 & 16.8 & 0.210 & 0.103 & $7.25_{-2.47}^{+3.06}$ \\
\hline $331.1-05.7$ & PC 11 & 5928675582979093376 & 1.00 & 249.42787 & -55.70736 & 11.8 & 0.032 & 0.233 & \\
\hline $331.3+16.8$ & NGC 5873 & 6199243477361421440 & 0.98 & 228.21189 & -38.12545 & 16.2 & -0.156 & 0.117 & $6.65_{-1.21}^{+1.30}$ \\
\hline $331.3-12.1$ & Hen 3-1357 & 5915762510400725760 & 0.98 & 259.08777 & -59.48987 & 15.4 & -3.501 & 0.746 & $6.36^{+1.56}$ \\
\hline $331.4+00.5$ & Pe 1-4 & 5983038839485182592 & 0.26 & 242.24509 & -51.03231 & 18.7 & 0.452 & 0.185 & \\
\hline $331.4-03.5$ & Hen 2-162 & 5933514606761839872 & 0.99 & 246.96216 & -54.02459 & 12.8 & -0.150 & 0.076 & \\
\hline $331.5-02.7$ & Hen 2-161 & 5933777110776156928 & 1.00 & 246.15773 & -53.37608 & 16.7 & 0.178 & 0.066 & $4.45_{-0.73}^{+0.85}$ \\
\hline $331.5-03.9$ & Hen 2-165 & 5933495227888260352 & 1.00 & 247.49880 & -54.15743 & 19.0 & 0.355 & 1.124 & $2.47_{-0.69}^{+8.70}$ \\
\hline $331.7-01.0$ & Mz 3 & 5934701559547878144 & 0.98 & 244.30577 & -51.98633 & 13.2 & 0.366 & 0.086 & \\
\hline $332.0+04.2$ & PHR J1556- & 5984598157441661952 & 0.47 & 239.15041 & -47.89465 & 20.3 & 1.046 & 0.681 & \\
\hline $332.0-03.3$ & Hen 2-164 & 5933557517709071872 & 0.98 & 247.47202 & -53.38737 & 17.8 & 0.281 & 0.113 & $3.75_{-0.81}^{+0.95}$ \\
\hline $332.0-04.3$ & PHR J1634-5405 & 5930511221989592448 & 0.82 & 248.58558 & -54.08445 & 19.2 & -1.249 & 0.519 & $\ldots$ \\
\hline $332.1+01.0$ & DeGaPe 64 & 5934781415925167104 & 0.91 & 244.76411 & -51.70235 & 20.7 & & & \\
\hline $332.1-05.8$ & PHR J1642-5501 & 5930256749426536064 & 0.45 & 250.73268 & -55.03109 & 17.9 & 0.955 & 0.123 & $\ldots$ \\
\hline $332.2+03.5$ & Wra 16-199 & 5984378392554625280 & 0.90 & 240.09181 & -48.25988 & 17.6 & 0.184 & 0.101 & \\
\hline $332.3+07.0$ & PHR J1547-4533 & 5988081337237556608 & 0.94 & 236.93698 & -45.54740 & 17.1 & 0.441 & 0.127 & $2.46_{-049}^{+0.61}$ \\
\hline $332.3-04.2$ & Hen 2-170 & 5930562899068086272 & 1.00 & 248.83846 & -53.83650 & 14.6 & & & \\
\hline $332.4-01.4$ & BMP J1622-5144 & 5934018728455510528 & 0.91 & 245.64181 & -51.74885 & 18.6 & 0.413 & 0.167 & \\
\hline $332.5-16.9$ & HaTr 7 & 5911656865276078080 & 1.00 & 268.53934 & -60.83274 & 14.8 & 0.587 & 0.034 & $1.65_{-0.09}^{+0.10}$ \\
\hline $332.8-16.4$ & HaTr 6 & 5911772863745103872 & 1.00 & 267.97007 & -60.38937 & 19.8 & 0.340 & 0.458 & $8.61_{-250}^{+2.50}$ \\
\hline $332.9-09.9$ & Hen 3-1333 & 5917195002285834240 & 0.99 & 257.25385 & -56.91332 & 11.4 & 0.678 & 0.047 & $1.45_{-0.09}^{+0.11}$ \\
\hline $333.2-00.6$ & PHR J1622-5038 & 5935234242865345280 & 0.34 & 245.66894 & -50.64462 & 20.9 & $\ldots$ & $\ldots$ & $\ldots$ \\
\hline $333.4+01.1$ & Pe $1-5$ & 5935593302133958528 & 0.24 & 243.83305 & -49.22217 & 20.1 & & & \\
\hline $333.4-04.0$ & HaTr 3 & 5931008441737475840 & 1.00 & 249.90624 & -52.82020 & 19.2 & 0.516 & 0.291 & \\
\hline $333.4-04.3$ & PHR J1641-5302 & 5930814309267559552 & 1.00 & 250.26825 & -53.04021 & 16.4 & 0.099 & 0.067 & $8.63_{-205}^{+2.32}$ \\
\hline $333.7-05.9$ & PHR J1650-5350 & 5929999296203805056 & 0.36 & 252.54944 & -53.83970 & 18.1 & 0.256 & 0.122 & \\
\hline $334.2-06.2$ & PHR J1654-5338 & 5929832131772573440 & 1.00 & 253.60439 & -53.64558 & 18.6 & -0.180 & 0.212 & \\
\hline $334.3-09.3$ & IC 4642 & 5923374773032038528 & 1.00 & 257.93834 & -55.40050 & 15.9 & 0.243 & 0.061 & $3.55_{-0.51}^{+0.62}$ \\
\hline $334.3-13.4$ & PHR J1736-5736 & 5918991329378768640 & 0.98 & 264.14017 & -57.60592 & 18.4 & 0.423 & 0.211 & \\
\hline $334.4+02.3$ & BMP J1614-4742 & 5989858079305553152 & 0.29 & 243.71748 & -47.70300 & 19.9 & 0.361 & 0.669 & . \\
\hline $334.6+01.3$ & Kn 82 & 5941689643134631424 & 0.63 & 245.06765 & -48.25626 & 20.8 & -0.856 & 1.695 & $\ldots$ \\
\hline $334.6-06.7$ & MPA J165 & 5935774927750122112 & 1.00 & 254.57100 & -53.69588 & 16.0 & 0.081 & 0.043 & 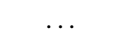 \\
\hline $334.8-03.6$ & BMP J1643-5129 & 5937189621284937216 & 0.85 & 250.84857 & -51.49133 & 16.4 & 0.084 & 0.055 & $\ldots$ \\
\hline $334.8-07.4$ & Hen 3-1312 & 5923760667266961408 & 0.99 & 255.76195 & -53.93168 & 11.1 & 0.159 & 0.052 & .. \\
\hline $334.9-12.8$ & PHR J1734-5649 & 5919316887904278528 & 1.00 & 263.74707 & -56.82350 & 20.4 & 0.467 & 0.728 & \\
\hline $335.2-03.6$ & HaTr 4 & 5937103069115240192 & 1.00 & 251.25075 & -51.20586 & 16.8 & 0.302 & 0.061 & $3.30_{-0.52}^{+0.67}$ \\
\hline $335.4+09.2$ & K 1-31 & 5995639350115110656 & 1.00 & 238.30185 & -41.84063 & 20.5 & 4.345 & 1.684 & $5.89_{-1.98}^{+0.1920}$ \\
\hline $335.4-01.1$ & Hen 2-169 & 5940494061671568896 & 0.78 & 248.55584 & -49.35340 & 22.0 & & & \\
\hline $335.5+12.4$ & DS 2 & 6008325614048284416 & 1.00 & 235.77093 & -39.30411 & 12.3 & 1.217 & 0.048 & $0.80^{+0.03}$ \\
\hline $335.6+01.7$ & Kn 83 & 5941903811721563008 & 0.80 & 245.57319 & -47.22916 & 15.4 & 0.305 & 0.034 & $\ldots$ \\
\hline $335.6-04.0$ & MeWe 1-8 & 5937073863334704896 & 1.00 & 252.16701 & -51.15544 & 17.8 & 0.012 & 0.122 & $\ldots$ \\
\hline $335.7+01.7$ & Kn 84 & 5941857288593503104 & 0.76 & 245.68426 & -47.20285 & 18.7 & 0.058 & 0.207 & $\cdots$ \\
\hline $335.8-01.6$ & MPA J1 & 5940454414906570624 & 0.96 & 249.50747 & -49.45528 & 20.1 & & & 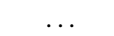 \\
\hline $335.9-01.3$ & MPA J1637-4911 & 5940883018248096640 & 0.93 & 249.30909 & -49.18778 & 17.2 & -0.057 & 0.109 & $\ldots$ \\
\hline $335.9-03.6$ & MeWe 1-7 & 5937506104459267840 & 0.99 & 251.98776 & -50.71327 & 18.4 & 0.001 & 0.181 & \\
\hline $336.0-00.4$ & PHR1633-4831 & 5941113533392568192 & 0.47 & 248.35879 & -48.53309 & 18.5 & 0.551 & 0.198 & \\
\hline $336.0-02.3$ & VRMF 104 & 5937798510171668480 & 0.96 & 250.52608 & -49.75159 & 18.3 & -0.155 & 0.187 & $\ldots$ \\
\hline $336.1+03.2$ & MPA J1618-4551 & 5990431204071731328 & 0.97 & 244.60710 & -45.85676 & 17.1 & 0.139 & 0.084 & \\
\hline $336.1+04.1$ & PM 2-17 & 5990663544616290304 & 0.21 & 243.76087 & -45.19810 & 22.0 & & & \\
\hline $336.2+01.9$ & Pe $1-6$ & 5942251600953774208 & 0.94 & 245.97736 & -46.70393 & 19.6 & -0.625 & 0.557 & $4.90_{-1.27}^{+1.32}$ \\
\hline $336.2-06.9$ & PC 14 & 5924318978646258304 & 0.97 & 256.56150 & -52.50016 & 16.9 & 0.464 & 0.224 & $5.33_{-1.84}^{+1.96}$ \\
\hline $336.3+08.0$ & PHR J1601-4209 & 5995171263094143360 & 0.94 & 240.25765 & -42.15000 & 19.0 & 0.196 & 0.308 & $\ldots$ \\
\hline $336.3+21.4$ & Pa J1519.5-3138 & 6207958343963986176 & 0.74 & 229.89692 & -31.63232 & 20.0 & -0.250 & 0.595 & \\
\hline $336.3-05.6$ & Hen 2-186 & 5936426147853199104 & 0.90 & 254.90028 & -51.70182 & 17.4 & & & $\cdots$ \\
\hline $336.5+05.5$ & MPA J1611-4356 & 5991591150152485376 & 0.99 & 242.80373 & -43.93945 & 17.6 & 0.183 & 0.115 & \\
\hline $336.5-05.8$ & MPA J1700-5136 & 5936471674477724160 & 1.00 & 255.24466 & -51.61187 & 19.0 & 0.297 & 0.255 & \\
\hline $336.8-07.2$ & K 2-17 & 5924383368793138560 & 0.95 & 257.39944 & -52.21734 & 17.2 & 0.094 & 0.083 & $5.64_{-1.07}^{+1.20}$ \\
\hline $336.9+08.3$ & WRAY 15-1407 & 5995280428294970880 & 1.00 & 240.55435 & -41.56003 & 15.4 & 0.144 & 0.044 & $\ldots$ \\
\hline
\end{tabular}


Table A.1: continued.

\begin{tabular}{|c|c|c|c|c|c|c|c|c|c|}
\hline PN G & PN name & Gaia source ID & reliability & $\begin{array}{c}\text { RA } \\
(\mathrm{deg})\end{array}$ & $\begin{array}{c}\text { Dec } \\
(\mathrm{deg})\end{array}$ & $\begin{array}{c}\mathrm{G} \\
(\mathrm{mag})\end{array}$ & $\begin{array}{c}\omega \\
(\mathrm{mas})\end{array}$ & $\begin{array}{c}\sigma_{\omega} \\
\text { (mas) }\end{array}$ & $\begin{array}{c}\text { distance } \\
(\mathrm{kpc})\end{array}$ \\
\hline $336.9-11.5$ & MeWe 1-10 & 5921422315225774336 & 1.00 & 263.61752 & -54.48270 & 18.8 & 0.665 & 0.235 & $3.41_{-114}^{+1.23}$ \\
\hline $337.0+08.4$ & PHR J1602-4127 & 5995289705424137472 & 0.98 & 240.57562 & -41.44712 & 16.7 & 1.497 & 0.081 & $0.66_{-0.03}^{+0.04}$ \\
\hline $337.1-05.2$ & PHR J1700-5047 & 5936569462322610688 & 0.85 & 255.15733 & -50.79001 & 17.3 & 0.110 & 0.115 & \\
\hline $337.2-04.6$ & MPA J1657-5017 & 5938282810641197184 & 0.92 & 254.49894 & -50.29217 & 18.9 & 0.474 & 0.241 & $\ldots$ \\
\hline $337.3+47.4$ & Fr 2-9 & 6328952378754074368 & 1.00 & 215.66797 & -9.28956 & 12.0 & 2.938 & 0.060 & $\ldots$ \\
\hline $337.3-02.3$ & MPA J1647-4846 & 5939408092078163712 & 0.82 & 251.82061 & -48.77985 & 19.9 & -0.258 & 0.693 & \\
\hline $337.4+01.6$ & Pe 1-7 & 5943697901113034368 & 0.97 & 247.60776 & -46.04748 & 15.6 & -0.319 & 0.160 & $\ldots$ \\
\hline $337.4+02.6$ & PHR J1625-4523 & 5942477172668108032 & 0.83 & 246.46192 & -45.37747 & 17.1 & 0.678 & 0.083 & $\ldots$ \\
\hline $337.4-09.1$ & Wray 16-266 & 5924816851223108864 & 1.00 & 260.65414 & -52.77630 & 17.8 & 0.408 & 0.131 & \\
\hline $337.5-05.1$ & Hen 2-187 & 5938103104921060224 & 1.00 & 255.40417 & -50.38266 & 12.5 & 0.204 & 0.021 & $4.21_{-0.34}^{+0.40}$ \\
\hline $337.8+01.5$ & MPA J1632-4547 & 5943683440033135616 & 0.44 & 248.03103 & -45.79951 & 18.2 & & & $\ldots .0 .34$ \\
\hline $337.9+43.5$ & Fr $1-5$ & 6324298665725984512 & 1.00 & 218.08641 & -12.38006 & 16.0 & 0.441 & 0.054 & $\ldots$ \\
\hline $338.0+02.4$ & PHR J1629-4505 & 5943952511091634048 & 0.63 & 247.27113 & -45.09591 & 21.0 & & & 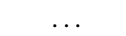 \\
\hline $338.1-04.8$ & PHR J1702-4943 & 5938219382596554240 & 0.73 & 255.67484 & -49.72259 & 20.5 & 1.787 & 1.502 & \\
\hline $338.1-08.3$ & NGC 6326 & 5925023250182476416 & 0.99 & 260.19335 & -51.75435 & 16.6 & 0.199 & 0.061 & $3.93_{-0.55}^{+0.63}$ \\
\hline $338.4+03.6$ & Kn 86 & 5992188940881265024 & 0.87 & 246.48239 & -43.96522 & 17.9 & 0.034 & 0.140 & -0.53 \\
\hline $338.4-02.0$ & MPA J1650-4747 & 5939391388953453696 & 0.97 & 252.54091 & -47.78469 & 20.6 & -1.580 & 1.430 & $\ldots$ \\
\hline $338.4-02.7$ & MPA J1653-4812 & 5939320062437196160 & 0.89 & 253.30147 & -48.20187 & 20.6 & & & $\ldots$ \\
\hline $338.5-02.1$ & Kn 87 & 5939388502731608704 & 0.76 & 252.79331 & -47.77850 & 20.2 & 0.374 & 0.721 & 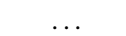 \\
\hline $338.6+01.1$ & BMP J1636-4529 & 5943035346640784000 & 0.95 & 249.24483 & -45.49144 & 18.9 & & & $\ldots$ \\
\hline $338.8+01.7$ & MPA J1635-4458 & 5943816996281726848 & 0.90 & 248.79317 & -44.97801 & 20.1 & & & \\
\hline $338.8+05.6$ & IC 4599 & 5992575625377163264 & 1.00 & 244.84670 & -42.26020 & 16.5 & 0.226 & 0.075 & $3.45_{-051}^{+0.59}$ \\
\hline $338.9+04.6$ & PHR J1624-4252 & 5992302744664872064 & 0.28 & 246.00337 & -42.88014 & 20.5 & 1.679 & 1.068 & $\ldots$ \\
\hline $339.1+03.0$ & MPA J1631-4354 & 5944477287388543360 & 0.94 & 247.77940 & -43.90857 & 19.7 & & & $\ldots$ \\
\hline $339.2+03.9$ & PHR J1627-4309 & 5944573151050162816 & 0.95 & 246.92174 & -43.15080 & 20.7 & & & $\ldots$ \\
\hline $339.4-03.7$ & PHR J1701-4803 & 5938982512368905344 & 0.86 & 255.45500 & -48.06402 & 19.5 & 0.104 & 0.536 & . \\
\hline $339.4-09.5$ & PHR J1721-5122 & 5925231465889406720 & 1.00 & 262.82857 & -51.36675 & 16.2 & 0.187 & 0.073 & \\
\hline $339.9+88.4$ & LoTr 5 & 3958428334589607552 & 1.00 & 193.89048 & +25.89184 & 8.6 & 2.198 & 0.047 & $0.45_{-0.01}^{+0.01}$ \\
\hline $340.0+02.9$ & PM 1-112 & 5944416569412103424 & 0.36 & 248.67999 & -43.29991 & 18.1 & 0.190 & 0.131 & \\
\hline $340.1-02.2 \mathrm{a}$ & MPA J1657-4633 & 5939874800405169024 & 0.94 & 254.27651 & -46.56656 & & -1.586 & 0.861 & $\ldots$ \\
\hline $340.2+06.4$ & PHR J1621-4040 & 5993876558082591104 & 0.99 & 245.43250 & -40.67334 & 19.7 & 0.375 & 0.458 & $\ldots$ \\
\hline $340.4-07.6$ & PHR J1724-4930 & 5949601561341432064 & 0.72 & 261.14463 & -49.51464 & 18.9 & 1.003 & 0.251 & .. \\
\hline $340.4-14.1$ & Sa $1-6$ & 6701733382321164928 & 0.99 & 270.24781 & -52.73889 & 16.0 & 0.123 & 0.048 & \\
\hline $340.8+10.8$ & Lo 12 & 5998464201638224640 & 1.00 & 242.10967 & -37.14654 & 19.6 & 0.863 & 0.378 & $2.56_{-0.85}^{+0.91}$ \\
\hline $340.8+12.3$ & Lo 11 & 6010827896356669824 & 1.00 & 240.84258 & -36.01494 & 18.6 & 0.247 & 0.198 & $3.79_{-0.91}^{+0.99}$ \\
\hline $340.9-04.6$ & WRAY 15-1633 & 5950881255412836608 & 0.55 & 257.86419 & -47.41722 & 18.7 & & & $\ldots$ \\
\hline $341.0-02.6$ & Kn 89 & 5963209701330219136 & 0.91 & 255.66466 & -46.13373 & 18.5 & -0.165 & 0.210 & \\
\hline $341.2-24.6$ & Lo 18 & 6643159820922279936 & 1.00 & 287.44914 & -55.58622 & 20.8 & 0.569 & 1.169 & $5.71_{-1.64}^{+1.64}$ \\
\hline $341.5+12.1$ & Sand 3 & 6010805807350513920 & 1.00 & 241.61864 & -35.75345 & 13.9 & 0.817 & 0.024 & \\
\hline $341.5-09.1$ & Hen 2-248 & 5946831685377720576 & 1.00 & 264.02892 & -49.42927 & 15.4 & -0.077 & 0.087 & \\
\hline $341.6+13.7$ & NGC 6026 & 6011169161583903488 & 1.00 & 240.33800 & -34.54328 & 13.1 & 0.345 & 0.019 & $2.59_{-0.12}^{+0.13}$ \\
\hline $341.6-03.8$ & Kn 91 & 5963338962666591104 & 0.49 & 257.54050 & -46.28270 & 15.9 & 0.130 & 0.041 & \\
\hline $341.7-01.3$ & Kn 92 & 5964462526103087488 & 0.27 & 254.76290 & -44.71346 & 21.0 & & & \\
\hline $341.7-06.0$ & SB 26 & 5950984089819027200 & 1.00 & 260.26225 & -47.59098 & 17.6 & 0.105 & 0.113 & \\
\hline $341.8+05.4$ & NGC 6153 & 6017034570775817984 & 0.93 & 247.87736 & -40.25352 & 15.3 & 0.719 & 0.046 & $1.32_{-0.07}^{+0.08}$ \\
\hline $341.8-05.6$ & Kn 93 & 5951003850951220480 & 0.48 & 259.85790 & -47.26322 & 20.1 & 0.211 & 0.631 & $\ldots$ \\
\hline $342.0-01.7$ & PHR J1702-4443 & 5963723654320289152 & 0.83 & 255.51794 & -44.72210 & 20.6 & 0.082 & 1.163 & \\
\hline $342.1+10.8$ & NGC 6072 & 6022549519257057280 & 1.00 & 243.24320 & -36.22983 & 18.6 & 1.086 & 0.200 & $1.03_{-0.17}^{+0.21}$ \\
\hline $342.1+27.5$ & Me 2-1 & 6251122593490273792 & 1.00 & 230.58031 & -23.62542 & 18.3 & -0.116 & 0.340 & $5.62_{-1.41}^{+1.47}$ \\
\hline $342.2-02.0$ & Kn 94 & 5963673901427848320 & 0.52 & 255.98732 & -44.83662 & 17.4 & 0.228 & 0.131 & $\ldots$ \\
\hline $342.3-06.0$ & SB 28 & 5950344590663972736 & 0.77 & 260.71873 & -47.04595 & 19.8 & 0.253 & 0.494 & \\
\hline $342.5-05.4$ & PHR J1720-4628 & 5951141186824828032 & 0.38 & 260.14683 & -46.55520 & 19.6 & -0.602 & 0.392 & \\
\hline $342.5-14.3$ & Sp 3 & 6702910375160462720 & 1.00 & 271.81582 & -51.01951 & 13.1 & -0.092 & 0.104 & $3.28_{-0.40}^{+0.44}$ \\
\hline $342.7-02.3$ & MPA J1706-4434 & 5963598821057669760 & 0.72 & 256.70565 & -44.57624 & 20.0 & 0.434 & 0.519 & $\ldots$ \\
\hline $342.8-06.6$ & Cn 1-4 & 5951803883085737216 & 0.84 & 261.95325 & -46.92820 & 17.0 & & & \\
\hline $342.9+05.6$ & Kn 96 & 6017471287373503616 & 0.88 & 248.62633 & -39.31711 & 18.7 & 0.239 & 0.279 & \\
\hline $342.9-02.0$ & Pe 1-8 & 5963798451140343936 & 0.60 & 256.59396 & -44.21972 & 19.6 & 0.118 & 0.354 & $2.33_{-0.52}^{+0.57}$ \\
\hline $342.9-02.3$ & WRAY 16-251 & 5963605319383850624 & 0.98 & 256.87751 & -44.38065 & 21.1 & & & $\ldots$ \\
\hline $343.0-01.7$ & $\mathrm{Vd} 1-9$ & 5965317327666432768 & 0.99 & 256.41217 & -43.93894 & 20.0 & & & $\ldots$ \\
\hline $343.2-10.8$ & BMP J1749-4848 & 5947532001244332032 & 1.00 & 267.45736 & -48.80893 & 15.3 & 0.043 & 0.052 & $\ldots$ \\
\hline $343.3+05.9$ & PHR J1634-3848 & 6017697919880338432 & 0.99 & 248.72517 & -38.80858 & 16.6 & 0.000 & 0.059 & \\
\hline $343.3-07.5$ & BMP J1733-4657 & 5951662286640985216 & 0.62 & 263.36568 & -46.96631 & 16.3 & 0.449 & 0.067 & \\
\hline $343.4+11.9$ & H 1-1 & 6022980081133498368 & 1.00 & 243.36738 & -34.59430 & 17.1 & 0.050 & 0.128 & $14.20_{-3.79}^{+3.90}$ \\
\hline $343.5-07.8$ & PC 17 & 5948679547781501184 & 0.84 & 263.92388 & -46.99704 & 15.2 & 0.184 & 0.055 & $\ldots$ \\
\hline $343.6+01.1$ & PHR J1654-4143 & 5966511431669118848 & 0.78 & 253.71222 & -41.73059 & 14.6 & 0.663 & 0.022 & \\
\hline $343.6+03.7$ & SuWt 3 & 5969207331126400384 & 0.98 & 251.10060 & -40.05566 & 18.8 & -0.111 & 0.283 & $6.54_{-1.77}^{+1.86}$ \\
\hline $343.6+03.7 \mathrm{a}$ & MPA J1644-4002 & 5969208087047630848 & 1.00 & 251.08510 & -40.03703 & 20.4 & -0.160 & 1.036 & $\ldots$ \\
\hline $343.9+00.8$ & H $1-5$ & 5966477793490248960 & 0.94 & 254.34896 & -41.63284 & 20.1 & -0.053 & 1.080 & 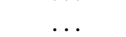 \\
\hline $343.9-05.8$ & SB 30 & 5951996160198892544 & 0.58 & 261.75955 & -45.54399 & 19.0 & & & \\
\hline $344.0+02.5$ & MPA J1650-4030 & 5969819892206749312 & 0.85 & 252.58417 & -40.50097 & 19.4 & 0.405 & 0.410 & $\cdots$ \\
\hline $344.2+01.6$ & IRAS $16515-4050$ & 5969663491005942912 & 0.96 & 253.75191 & -40.92628 & 20.6 & & & \\
\hline $344.2+04.7$ & Vd 1-1 & 5971001081288748032 & 0.98 & 250.63935 & -38.90890 & 15.3 & -0.124 & 0.156 & $\ldots$ \\
\hline
\end{tabular}


Table A.1: continued.

\begin{tabular}{|c|c|c|c|c|c|c|c|c|c|}
\hline PN G & PN name & Gaia source ID & reliability & $\begin{array}{c}\text { RA } \\
(\mathrm{deg})\end{array}$ & $\begin{array}{c}\text { Dec } \\
(\mathrm{deg})\end{array}$ & $\begin{array}{c}\mathrm{G} \\
(\mathrm{mag})\end{array}$ & $\begin{array}{c}\omega \\
(\mathrm{mas})\end{array}$ & $\begin{array}{c}\sigma_{\omega} \\
\text { (mas) }\end{array}$ & $\begin{array}{c}\text { distance } \\
(\mathrm{kpc})\end{array}$ \\
\hline $344.2-01.2$ & H 1-6 & 5965494928876093824 & 0.57 & 256.74622 & -42.68569 & 18.6 & 0.708 & 0.194 & $\ldots$ \\
\hline $344.4+01.8$ & MPA J1654-4041 & 5969680670842881664 & 0.98 & 253.67992 & -40.69625 & 19.8 & 1.964 & 0.922 & . \\
\hline $344.4+02.8$ & Vd 1-5 & 5969867381677383552 & 0.46 & 252.89007 & -40.04856 & 20.4 & $\ldots$ & $\ldots$ & $\ldots$ \\
\hline $344.4-03.5$ & MPA J1717-4351 & 5953484898925238272 & 0.98 & 259.47181 & -43.86617 & 21.1 & & & $\ldots$ \\
\hline $344.4-06.1$ & Wray $16-278$ & 5952198676546036096 & 1.00 & 262.51615 & -45.38078 & 16.6 & 0.170 & 0.080 & . \\
\hline $344.5-12.5$ & Kn 99 & 6707279971809736960 & 1.00 & 270.75778 & -48.49457 & 13.4 & 0.125 & 0.030 & $\ldots$ \\
\hline $344.6-04.5$ & MPA J1723-4419 & 5953237508824133376 & 0.90 & 260.77521 & -44.32128 & 18.7 & 0.056 & 0.227 & 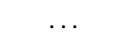 \\
\hline $344.8+03.4$ & Vd 1-3 & 5970789253440454016 & 0.97 & 252.38699 & -39.35257 & 17.8 & 0.274 & 0.130 & $\ldots$ \\
\hline $344.8+06.2$ & PKP J1638-3731 & 6019357224689844096 & 1.00 & 249.69173 & -37.52860 & 18.5 & 0.066 & 0.201 & $\ldots$ \\
\hline $344.8-02.6$ & MPA J1715-4303 & 5953602718496619776 & 0.92 & 258.81646 & -43.06505 & 19.9 & -0.013 & 0.596 & $\ldots$ \\
\hline $344.8-03.5$ & MPA J1719-4336 & 5953333539982068736 & 0.90 & 259.80694 & -43.60963 & 19.2 & 0.121 & 0.281 & \\
\hline $344.9+03.0$ & BMP J1651-3930 & 5970024611844351616 & 1.00 & 252.92211 & -39.50765 & 19.0 & 0.615 & 0.213 & $1.52_{-027}^{+0.32}$ \\
\hline $344.9-01.9$ & PM 1-131 & 5965643642122666880 & 0.91 & 258.09186 & -42.51153 & 19.7 & 0.723 & 0.597 & $\ldots$ \\
\hline $345.0+03.4$ & Vd 1-4 & 5970798427549713792 & 0.93 & 252.60563 & -39.13864 & 17.2 & & & \\
\hline $345.0+04.3$ & Vd 1-2 & 5971070556654899456 & 0.99 & 251.68807 & -38.61613 & 14.6 & -0.017 & 0.054 & \\
\hline $345.0-04.9$ & Cn 1-3 & 5952577144700811136 & 0.99 & 261.55130 & -44.19043 & 13.6 & -0.333 & 0.228 & $8.99_{-219}^{+2.30}$ \\
\hline $345.1-13.6$ & StWr 2-37 & 6706413247400610048 & 1.00 & 272.64709 & -48.43009 & 16.4 & 0.067 & 0.138 & \\
\hline $345.2-01.2$ & H 1-7 & 5965810351256669952 & 0.99 & 257.61409 & -41.88040 & 17.0 & 0.958 & 0.095 & $1.05_{-010}^{+0.12}$ \\
\hline $345.2-08.8$ & IC 1266 & 5954912374289120896 & 1.00 & 266.39702 & -46.08996 & 11.3 & 0.267 & 0.034 & $3.12_{-0.28}^{+0.33}$ \\
\hline $345.3-10.2$ & MeWe 1-11 & 5954082414802884736 & 0.64 & 268.19623 & -46.69969 & 17.7 & 0.191 & 0.112 & $4.73_{-1.03}^{+1.16}$ \\
\hline $345.4+00.1$ & IC 4637 & 5966769881320062208 & 0.99 & 256.29363 & -40.88557 & 12.5 & 0.693 & 0.015 & $1.38_{-0.03}^{+0.03}$ \\
\hline $345.4+04.0$ & Kn 100 & 5970911127486802816 & 0.91 & 252.25673 & -38.46201 & 17.1 & 1.504 & 0.229 & $\ldots$ \\
\hline $345.4-02.3$ & $\mathrm{CBF} 3$ & 5953717720528712704 & 0.31 & 258.94526 & -42.40189 & 19.4 & 1.151 & 0.418 & \\
\hline $345.5+15.1$ & Lo 13 & 6036783899864816768 & 1.00 & 242.44141 & -30.91883 & 17.8 & 0.414 & 0.127 & $3.63_{-0.95}^{+1.17}$ \\
\hline $345.5-08.1$ & Kn 101 & 5955009955937563136 & 0.98 & 265.82551 & -45.47803 & 19.0 & 1.121 & 0.253 & \\
\hline $345.6+05.1$ & Pa J1645.4-3737 & 5971174421816752000 & 0.98 & 251.36865 & -37.61904 & 18.2 & 0.403 & 0.145 & .. \\
\hline $345.6+06.7$ & Hen 2-175 & 6019559637912934912 & 0.99 & 249.86712 & -36.57128 & 19.2 & & & \\
\hline $345.6-07.4$ & MPA J1740-4504 & 5955219790876459264 & 1.00 & 265.02631 & -45.07264 & 18.9 & 0.881 & 0.263 & \\
\hline $345.9+03.0$ & Vd 1-6 & 5970446510755965440 & 0.44 & 253.61401 & -38.73630 & 19.0 & 0.398 & 0.271 & $3.71_{-1.33}^{+1.60}$ \\
\hline $346.1+02.8$ & MPA J1656-3841 & 5970420676566203392 & 0.97 & 254.00730 & -38.69741 & 20.0 & 0.483 & 0.507 & \\
\hline $346.2-05.5$ & Kn 103 & 5958481320307081472 & 1.00 & 263.13916 & -43.49477 & 18.9 & 0.052 & 0.245 & \\
\hline $346.2-08.2$ & IC 4663 & 5955085238132418048 & 0.99 & 266.36929 & -44.90502 & 16.6 & 0.386 & 0.079 & $2.87_{-0.50}^{+0.66}$ \\
\hline $346.5+02.7$ & PPA J1657-3826 & 5970512829351531520 & 0.90 & 254.43689 & -38.44672 & 20.3 & -0.127 & 0.908 & \\
\hline $346.5+13.5$ & PHR J1618-3119 & 6037404849053055488 & 1.00 & 244.62596 & -31.33098 & 14.8 & 0.228 & 0.030 & \\
\hline $346.7-04.4$ & Sa 2-202 & 5958744133656575232 & 0.99 & 262.29154 & -42.54598 & 16.3 & -0.003 & 0.107 & \\
\hline $346.8-00.7$ & MPA J1713-4015 & 5972109938708943360 & 0.85 & 258.29517 & -40.26559 & 20.8 & & & $\ldots$ \\
\hline $346.9+12.4$ & Abell 38 & 6025240677023518336 & 0.92 & 245.82919 & -31.74962 & 19.7 & -0.294 & 0.402 & \\
\hline $346.9-08.9$ & PHR J1750-4438 & 5954720062822377216 & 1.00 & 267.69057 & -44.63943 & 17.8 & 0.070 & 0.146 & $\ldots$ \\
\hline $347.0+12.9$ & BMP J1621-3121 & 6037321732830715264 & 0.66 & 245.40793 & -31.35785 & 19.9 & 0.350 & 0.515 & $\ldots$ \\
\hline $347.3+05.0$ & PHR J1651-3621 & 5971717997197736704 & 0.90 & 252.86172 & -36.35999 & 19.2 & 0.108 & 0.307 & \\
\hline $347.4+01.6$ & PPA J1704-3824 & 5973285041764508032 & 0.78 & 256.24639 & -38.40124 & 20.8 & 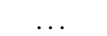 & $\ldots$ & $\ldots$ \\
\hline $347.4+05.8$ & H 1-2 & 6019844995543616256 & 0.99 & 252.22544 & -35.78582 & 14.7 & & $\ldots$ & $\ldots$ \\
\hline $347.6-02.8$ & RPZM 15 & 5960049533140204800 & 0.21 & 261.16841 & -40.87233 & 21.0 & & & . \\
\hline $347.6-14.5$ & Kn 105 & 6707772449939066240 & 1.00 & 275.33478 & -46.56157 & 16.2 & 0.353 & 0.048 & $\ldots$ \\
\hline $347.7+02.0$ & Vd 1-8 & 5976336220892300032 & 0.99 & 256.14078 & -37.88746 & 19.1 & & $\ldots$ & $\ldots$ \\
\hline $347.7-00.7$ & RPZM 8 & 5972248958280794240 & 0.57 & 258.96506 & -39.55223 & 20.9 & & & \\
\hline $347.8-03.9$ & Kn 106 & 5959225105593657856 & 0.54 & 262.56643 & -41.36030 & 18.7 & 0.325 & 0.191 & $\ldots$ \\
\hline $347.9-06.0$ & SB 31 & 5955984123273721984 & 0.83 & 265.01407 & -42.40165 & 18.5 & -0.136 & 0.281 & \\
\hline $348.0+06.3$ & WRAY 15-1537 & 6020091664096478976 & 0.68 & 252.20219 & -35.01617 & 19.6 & -0.062 & 0.787 & \\
\hline $348.0-13.8$ & IC 4699 & 6708047083020633472 & 1.00 & 274.63352 & -45.98383 & 15.3 & 0.150 & 0.050 & $6.01_{-1.14}^{+1.40}$ \\
\hline $348.4+04.9$ & MPA J1655-3535 & 5977790526875496064 & 0.99 & 253.84188 & -35.58998 & 16.3 & -0.041 & 0.091 & ${ }^{-1.14}$ \\
\hline $348.4-04.1$ & H $1-21$ & 5959304437876436224 & 0.97 & 263.19920 & -40.97465 & 16.9 & 0.693 & 0.156 & . \\
\hline $348.5-04.0$ & Kn 109 & 5959362063504880000 & 0.36 & 263.16821 & -40.74954 & 20.7 & & & 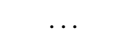 \\
\hline $348.7-02.5$ & MPA J1726-3950 & 5960387839148474368 & 0.32 & 261.64282 & -39.83921 & 19.8 & 0.689 & 0.481 & $\ldots$ \\
\hline $348.8-09.0$ & Hen 2-306 & 5956267659817411712 & 0.98 & 269.14045 & -43.05535 & 15.5 & 0.135 & 0.061 & \\
\hline $348.9+04.6$ & KLSS $1-15$ & 5977832621856619392 & 0.87 & 254.48803 & -35.41494 & 20.3 & -2.111 & 0.879 & $\ldots$ \\
\hline $349.2-03.5$ & H 2-14 & 5961654029849113728 & 0.91 & 263.08368 & -39.85629 & 18.2 & 0.362 & 0.175 & \\
\hline $349.3+02.4$ & K 6-18 & 5976931365924778624 & 0.33 & 256.86909 & -36.39526 & 21.1 & & & $\ldots$ \\
\hline $349.3+07.4$ & PHR J1648-3318 & 6027007008100377856 & 1.00 & 252.18831 & -33.31570 & 19.6 & 0.344 & 0.394 & $\cdots$ \\
\hline $349.3-00.5$ & Pre 68 & 5972784416074384640 & 0.75 & 259.96432 & -38.16089 & 17.4 & 0.373 & 0.098 & \\
\hline $349.3-00.6$ & PreMo 2 & 5972785176284635904 & 0.97 & 260.15160 & -38.21379 & 21.3 & & & \\
\hline $349.3-01.1$ & NGC 6337 & 5972577055062637056 & 0.91 & 260.56530 & -38.48383 & 15.6 & 0.594 & 0.039 & $1.57_{-0.09}^{+0.10}$ \\
\hline $349.3-04.2$ & Lo 16 & 5960852520245103744 & 0.55 & 263.92450 & -40.19070 & 16.0 & 0.549 & 0.045 & $1.67_{-0.11}^{+0.12}$ \\
\hline $349.5+02.0$ & PHR J1709-3629 & 5976873641579140608 & 0.37 & 257.39154 & -36.49011 & 21.1 & & & $\ldots .11$ \\
\hline $349.6+03.1$ & PHR J1706-3544 & 5977201364758715136 & 0.85 & 256.50777 & -35.74349 & 20.6 & & & \\
\hline $349.7+06.2$ & PPA J1654-3343 & 6026276240884866432 & 0.99 & 253.53443 & -33.73298 & 19.2 & -0.124 & 0.310 & \\
\hline $349.8+04.4$ & M 2-4 & 5978248095516721280 & 0.97 & 255.27591 & -34.82741 & 16.7 & 0.141 & 0.155 & $7.67_{-2.11}^{+2.23}$ \\
\hline $349.9+02.6$ & WRAY 15-1624 & 5977166249135940096 & 0.84 & 257.14250 & -35.80188 & 20.6 & & & \\
\hline $350.1-03.9$ & H $1-26$ & 5961023459912201088 & 0.90 & 264.12458 & -39.36578 & 16.8 & 0.010 & 0.092 & $3.76_{-0.52}^{+0.57}$ \\
\hline $350.3+06.4$ & MPA J1655-3310 & 6026680968535085440 & 0.97 & 253.75526 & -33.16982 & 18.6 & 0.167 & 0.197 & \\
\hline $350.4+07.6$ & PM 1-115 & 6027575936660117888 & 0.98 & 252.77636 & -32.38348 & 18.0 & & & $\ldots$ \\
\hline $350.5+03.0$ & K 5-24 & 5977294509695487104 & 0.80 & 257.26795 & -35.08807 & 20.2 & -2.198 & 0.970 & $\cdots$ \\
\hline
\end{tabular}


Table A.1: continued.

\begin{tabular}{|c|c|c|c|c|c|c|c|c|c|}
\hline $\mathrm{PN} \mathrm{G}$ & PN name & Gaia source ID & reliability & $\begin{array}{c}\text { RA } \\
(\mathrm{deg})\end{array}$ & $\begin{array}{c}\text { Dec } \\
(\mathrm{deg})\end{array}$ & $\begin{array}{c}\mathrm{G} \\
(\mathrm{mag})\end{array}$ & $\begin{array}{c}\omega \\
(\mathrm{mas})\end{array}$ & $\begin{array}{c}\sigma_{\omega} \\
(\mathrm{mas})\end{array}$ & $\begin{array}{c}\text { distance } \\
(\mathrm{kpc})\end{array}$ \\
\hline $350.8-02.4$ & H 1-22 & 5962119947915694976 & 0.99 & 263.09229 & -37.95661 & 18.0 & 0.678 & 0.197 & $3.00_{-140}^{+3.78}$ \\
\hline $350.8-03.6$ & MPA J1737-3837 & 5961828950941856896 & 0.68 & 264.27178 & -38.62157 & 19.1 & -0.820 & 0.459 & $\ldots$ \\
\hline $350.8-07.4$ & Kn 110 & 5957121808565782144 & 1.00 & 268.57226 & -40.61717 & 19.6 & -0.223 & 0.374 & \\
\hline $350.9+04.4$ & H 2-1 & 5978397113707361536 & 0.98 & 256.15103 & -33.98856 & 13.0 & 0.340 & 0.025 & $2.70_{-0.17}^{+0.20}$ \\
\hline $350.9-03.7$ & PHR J1738-3839 & 5961451925866334720 & 0.67 & 264.50221 & -38.65395 & 20.4 & . & $\ldots$ & -0.17 \\
\hline $351.0+05.8$ & PHR J1659-3258 & 6026595515973923712 & 0.45 & 254.85661 & -32.97617 & 20.9 & & & \\
\hline $351.0-10.4$ & HaTr 9 & 6725198815882935168 & 1.00 & 272.24535 & -41.81042 & 18.2 & 1.189 & 0.193 & $1.02_{-018}^{+0.27}$ \\
\hline $351.1+02.6$ & PHR J1712-3452 & 5978810843677667840 & 0.61 & 258.07932 & -34.86868 & 18.2 & 0.672 & 0.173 & \\
\hline $351.1+04.8$ & M 1-19 & 5978493325390055296 & 0.96 & 255.94517 & -33.49570 & 16.1 & 0.102 & 0.071 & $6.83_{-1.40}^{+1.59}$ \\
\hline $351.2+05.2$ & M 2-5 & 5978493286612599424 & 0.94 & 255.57953 & -33.16804 & 15.9 & -0.080 & 0.105 & $7.03_{-1.44}^{+1.50}$ \\
\hline $351.3+07.6$ & H 1-4 & 6027623799797947776 & 1.00 & 253.40447 & -31.67589 & 15.6 & 0.201 & 0.092 & \\
\hline $351.5+02.5$ & PM 1-134 & 5978633684806915328 & 0.82 & 258.43085 & -34.64070 & 19.4 & -0.314 & 0.424 & \\
\hline $351.5-06.5$ & SB 34 & 4036440466993936640 & 0.99 & 268.03923 & -39.53750 & 18.0 & -0.145 & 0.138 & $10.38^{+2.49}$ \\
\hline $351.6-06.2$ & H 1-37 & 5958174178599344768 & 0.51 & 267.68581 & -39.29074 & 19.3 & -0.021 & 0.435 & \\
\hline $351.7-06.6$ & SB 35 & 4036448129216710272 & 0.96 & 268.26196 & -39.40252 & 15.6 & 0.106 & 0.037 & $8.46_{-1.85}^{+2.44}$ \\
\hline $351.7-10.9$ & Wray $16-385$ & 6724822782927554176 & 1.00 & 273.22057 & -41.50744 & 16.2 & 0.044 & 0.077 & -1. \\
\hline $351.9+02.4$ & K 5-26 & 5978668800481153792 & 0.66 & 258.75022 & -34.29384 & 20.5 & & & - \\
\hline $351.9+03.9$ & PHR J1709-3327 & 5979760271980432384 & 0.74 & 257.34093 & -33.46301 & 20.5 & & & \\
\hline $351.9+09.0$ & PC 13 & 6031037577201941888 & 1.00 & 252.57115 & -30.33203 & 16.6 & 0.122 & 0.082 & $7.33_{-1.67}^{+1.85}$ \\
\hline $351.9-01.9$ & Wray $16-286$ & 5974600744893097472 & 0.84 & 263.25281 & -36.73137 & 18.5 & 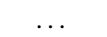 & $\ldots$ & $\ldots$ \\
\hline $352.0-04.6$ & H $1-30$ & 5961349568244600192 & 0.71 & 266.27821 & -38.14726 & 18.6 & & 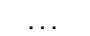 & $\ldots$ \\
\hline $352.1+05.1$ & M 2-8 & 5980037005287502976 & 0.22 & 256.37807 & -32.53540 & 18.6 & & & $\ldots$ \\
\hline $352.1-02.6$ & PHR J17 & 5962549410293600128 & 0.41 & 264.07190 & -36.99645 & 15.6 & 0.225 & 0.038 & - \\
\hline $352.2+14.6$ & Kn J1631.3-2629 & 6045582879087358720 & 0.41 & 247.84933 & -26.49297 & 19.8 & -0.646 & 0.802 & $\ldots$ \\
\hline $352.2-04.3$ & PHR J1743-3749 & 5961559158294341760 & 0.71 & 265.95420 & -37.83004 & 19.1 & 0.645 & 0.286 & \\
\hline $352.2-05.1$ & PHR J1747-3811 & 4039801437840555648 & 0.35 & 266.91649 & -38.19534 & 20.2 & 0.316 & 0.808 & - \\
\hline $352.3+12.2$ & MPA J1640-2757 & 6032515045967171072 & 1.00 & 250.04385 & -27.95930 & 18.6 & -0.641 & 0.249 & $\ldots$ \\
\hline $352.4+04.0$ & WRAY 16-255 & 5979871322676296192 & 0.98 & 257.55718 & -32.98082 & 14.7 & 0.067 & 0.032 & \\
\hline $352.4-02.7$ & PPA J1737-3650 & 5962633587289077504 & 0.64 & 264.40685 & -36.83842 & 18.3 & -0.381 & 0.193 & \\
\hline $352.6+00.1$ & H 1-12 & 5975747702989704064 & 0.99 & 261.60121 & -35.02818 & 19.1 & 0.149 & 0.331 & $2.69_{-0.67}^{+0.75}$ \\
\hline $352.6+02.2$ & PPA J1717-3349 & 5978801356020553344 & 0.83 & 259.48437 & -33.82414 & 19.5 & -1.233 & 0.582 & $\ldots$ \\
\hline $352.6-04.9$ & SB 37 & 4039816242659008256 & 0.56 & 266.96957 & -37.80077 & 19.4 & -2.546 & 1.254 & \\
\hline $352.7+02.6$ & PM 1-136 & 5978994359012496000 & 0.61 & 259.10908 & -33.55664 & 20.3 & & & $\ldots$ \\
\hline $352.7-01.7$ & PPA J1734-3600 & 5974750244145839744 & 0.97 & 263.59283 & -36.01323 & 19.2 & & & $\cdots$ \\
\hline $352.7-02.5$ & PPA J1737-3627 & 5962683516372855424 & 0.90 & 264.40257 & -36.45164 & 17.7 & 0.012 & 0.127 & \\
\hline $352.7-08.4$ & SB 38 & 4035884049752727808 & 1.00 & 270.87169 & -39.35783 & 17.2 & 0.050 & 0.110 & $\ldots$ \\
\hline $352.8+03.0$ & PPA J1715-3313 & 5979063834398849024 & 0.72 & 258.79339 & -33.22986 & 21.0 & & & \\
\hline $352.8+08.4$ & MPA J1654-3000 & 6029558076932364672 & 1.00 & 253.71922 & -30.01448 & 18.2 & -0.082 & 0.164 & \\
\hline $352.8-00.2$ & H $1-13$ & 5975007147549090432 & 1.00 & 262.11491 & -35.12547 & 18.3 & 0.591 & 0.229 & $1.76_{-0.39}^{+0.48}$ \\
\hline $352.8-04.6$ & PPA J1746-3725 & 4040049957552482560 & 0.43 & 266.74873 & -37.42677 & 17.1 & -0.011 & 0.095 & \\
\hline $352.9+02.6$ & PPA J1717-3323 & 5979377397030664960 & 0.46 & 259.34798 & -33.39833 & 17.5 & 1.311 & 0.126 & \\
\hline $352.9+11.4$ & K 2-16 & 6033785806546839936 & 1.00 & 251.20437 & -28.06799 & 12.1 & 0.175 & 0.024 & $4.82_{-0.50}^{+0.62}$ \\
\hline $352.9-07.5$ & $\mathrm{Fg} 3$ & 4035783611879028736 & 0.98 & 270.04922 & -38.83129 & 14.1 & -0.214 & 0.206 & $6.34_{-1.41}^{+1.50}$ \\
\hline $352.9-09.8$ & WRAY 16-376 & 6726004757944446848 & 1.00 & 272.65646 & -39.88740 & 15.0 & -0.037 & 0.040 & $\ldots$ \\
\hline $353.0+08.3$ & MyCn 26 & 6029584057264295168 & 0.85 & 253.94703 & -29.83850 & 15.7 & & & \\
\hline $353.2+05.7$ & K 5-22 & 6028295326608856832 & 0.98 & 256.54711 & -31.30075 & 18.5 & 0.098 & 0.225 & $\ldots$ \\
\hline $353.2-10.4$ & Kn 74 & 6725816913248112128 & 1.00 & 273.54523 & -39.92316 & 16.9 & 0.271 & 0.081 & $\ldots$ \\
\hline $353.3+06.3$ & M 2-6 & 6028519485121240832 & 0.62 & 256.07637 & -30.89138 & 16.3 & 0.066 & 0.095 & $\ldots$ \\
\hline $353.3-01.1$ & RPZM 23 & 5975047043518702208 & 0.89 & 263.34440 & -35.18452 & 21.0 & $x_{-1}+2$ & $\ldots$ & $\ldots$ \\
\hline $353.3-02.9$ & PHR J1740-3607 & 4041146552967012864 & 0.61 & 265.20919 & -36.12968 & 20.9 & & & \\
\hline $353.3-08.3$ & SB 39 & 4036109209068689024 & 0.90 & 271.13258 & -38.79375 & 18.0 & 0.573 & 0.155 & $2.66_{-075}^{+1.04}$ \\
\hline $353.4-04.5$ & K 6-13 & 4040170633255266816 & 0.93 & 267.00393 & -36.83553 & 19.9 & -0.158 & 0.611 & \\
\hline $353.5+06.9$ & K 5-21 & 6028619407659071232 & 1.00 & 255.64603 & -30.32085 & 18.8 & -0.022 & 0.224 & $\ldots$ \\
\hline $353.5-03.3$ & IRAS $17393-3612$ & 4040965648903119488 & 0.26 & 265.71080 & -36.22624 & 20.8 & & & \\
\hline $353.5-05.0$ & JaFu 2 & 4039968048311942400 & 0.27 & 267.54514 & -37.05707 & 16.4 & 2.201 & 0.446 & $0.51_{-010}^{+0.21}$ \\
\hline $353.6-01.3$ & MPA J1735-3502 & 4053328935619251200 & 0.74 & 263.81986 & -35.04723 & 17.8 & 0.142 & 0.140 & \\
\hline $353.6-02.6$ & PN RPZM 31 & 4041193591374943360 & 0.89 & 265.18997 & -35.73276 & 17.7 & 0.125 & 0.173 & $\ldots$ \\
\hline $353.6-03.6$ & K 6-11 & 4040919984780241024 & 0.73 & 266.14826 & -36.23402 & 17.4 & 0.242 & 0.121 & $\ldots$ \\
\hline $353.7+06.3$ & M 2-7 & 6028553986629499136 & 0.98 & 256.30795 & -30.53878 & 17.2 & & & 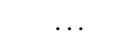 \\
\hline $353.7-01.1$ & PPA J1734-3449 & 4053525713754450816 & 0.59 & 263.62617 & -34.81754 & 18.7 & -0.186 & 0.289 & \\
\hline $353.7-12.8$ & Wray $16-411$ & 6726303683327171200 & 1.00 & 276.67378 & -40.49808 & 17.3 & 0.207 & 0.092 & $5.91_{-143}^{+1.62}$ \\
\hline $353.9+02.7$ & PM 1-140 & 5979532810495670784 & 0.98 & 259.93939 & -32.50897 & 19.9 & 0.876 & 0.759 & \\
\hline $353.9+03.6$ & PHR J1715-3159 & 5980277660869506048 & 0.47 & 258.95132 & -31.98935 & 20.3 & 0.312 & 0.936 & $\cdots$ \\
\hline $354.0+04.8$ & MPA J1711-3112 & 5980610190085763456 & 0.72 & 257.90439 & -31.20599 & 17.1 & -0.734 & 0.262 & \\
\hline $354.0-01.3$ & PPA J1736-3444 & 4053343121809563904 & 0.54 & 264.02382 & -34.73522 & 18.1 & 0.007 & 0.193 & \\
\hline $354.2+04.3$ & M 2-10 & 5980550511636230656 & 0.53 & 258.52925 & -31.32863 & 17.2 & 0.378 & 0.101 & $3.60_{-1.04}^{+1.82}$ \\
\hline $354.3-06.8$ & K 5-38 & 4037053105525701248 & 0.60 & 270.01825 & -37.28365 & 18.8 & 0.345 & 0.301 & $\ldots$ \\
\hline $354.4+05.4$ & K 6-21 & 6028694758571537664 & 0.88 & 257.68085 & -30.54121 & 21.0 & & & \\
\hline $354.4-07.8$ & H $1-52$ & 4036334471597172352 & 0.94 & 271.24005 & -37.63543 & 19.5 & 0.270 & 0.396 & $\cdots$ \\
\hline $354.5+03.3$ & Th $3-4$ & 5980371256845517056 & 0.92 & 259.71641 & -31.65184 & 18.2 & & & \\
\hline $354.5-02.0$ & PPA J17 & 4053388996355024768 & 0.60 & 265.03640 & -34.63750 & 20.4 & & & \\
\hline $354.5-02.0 \mathrm{a}$ & PPA J1740-3437 & 4053394700090930688 & 0.85 & 265.12721 & -34.62149 & 20.7 & 0.231 & 1.386 & $\ldots$ \\
\hline
\end{tabular}


Table A.1: continued.

\begin{tabular}{|c|c|c|c|c|c|c|c|c|c|}
\hline $\mathrm{PN} \mathrm{G}$ & PN name & Gaia source ID & reliability & $\begin{array}{c}\text { RA } \\
(\text { deg })\end{array}$ & $\begin{array}{c}\text { Dec } \\
(\mathrm{deg})\end{array}$ & $\begin{array}{c}\mathrm{G} \\
(\mathrm{mag})\end{array}$ & $\begin{array}{c}\omega \\
(\mathrm{mas})\end{array}$ & $\begin{array}{c}\sigma_{\omega} \\
(\mathrm{mas})\end{array}$ & $\begin{array}{l}\text { distance } \\
(\mathrm{kpc})\end{array}$ \\
\hline $354.5-03.9$ & Sab 41 & 4041043675655024128 & 0.53 & 267.06814 & -35.64182 & 17.7 & 0.789 & 0.131 & $1.46^{+0.36}$ \\
\hline $354.6+04.9$ & Terz N 139 & 5980734233101949696 & 0.29 & 258.22279 & -30.66898 & 20.1 & & & $\ldots$ \\
\hline $354.6+05.2$ & PM 1-130 & 6028777801634423808 & 0.69 & 257.92058 & -30.48045 & 14.7 & 0.130 & 0.059 & \\
\hline $354.6-01.4$ & PPA J1737-3414 & 4053473585749584384 & 0.74 & 264.47466 & -34.24101 & 20.3 & -4.024 & 1.230 & $\ldots$ \\
\hline $354.6-04.5$ & PPA J1750-3548 & 4040652696144746752 & 0.26 & 267.73395 & -35.81385 & 19.5 & -7.033 & 1.391 & $\ldots$ \\
\hline $354.6-11.4$ & $\mathrm{~Pa} 48$ & 6726675833653416832 & 1.00 & 275.37430 & -39.13824 & 14.9 & -0.845 & 0.249 & $\ldots$ \\
\hline $354.7+00.3$ & PPA J1731-3312 & 4054490672673910016 & 0.94 & 262.78231 & -33.20388 & 16.6 & 0.831 & 0.076 & $\ldots$ \\
\hline $354.7-03.9$ & MPA J1748-3530 & 4041421362193585152 & 0.61 & 267.20261 & -35.50814 & 19.4 & 0.681 & 0.919 & $\ldots$ \\
\hline $354.7-07.2$ & SB 40 & 4037160548451676416 & 0.48 & 270.73220 & -37.13733 & 17.6 & 0.342 & 0.146 & $\ldots$ \\
\hline $354.7-10.0$ & SB 41 & 6727598809253337088 & 1.00 & 273.91268 & -38.46570 & 18.4 & 0.363 & 0.197 & $\ldots$ \\
\hline $354.8+01.6$ & PHR J1726-3221 & 4057770309694460032 & 0.98 & 261.60747 & -32.36387 & 15.2 & 0.705 & 0.078 & $\ldots$ \\
\hline $354.8+04.2$ & K 5-28 & 5980676371346246144 & 0.28 & 259.05943 & -30.92682 & 17.2 & -0.046 & 0.089 & $\ldots$ \\
\hline $354.8+05.1$ & PHR J1712-3021 & 6028785605595920768 & 0.62 & 258.16288 & -30.36081 & 20.1 & & & $\ldots$ \\
\hline $354.9+03.5$ & Th 3-6 & 5980423277494359040 & 0.80 & 259.83432 & -31.21138 & 19.4 & 0.438 & 0.447 & $\ldots$ \\
\hline $354.9-05.7$ & K 5-36 & 4040437604099840896 & 0.98 & 269.25259 & -36.25452 & 18.5 & 0.208 & 0.205 & $\ldots$ \\
\hline $355.0-03.7$ & K 5-18 & 4041450327354434304 & 0.93 & 267.12329 & -35.09133 & 19.6 & -0.188 & 0.785 & $\ldots$ \\
\hline $355.0-06.1$ & PN PM 1-196 & 4037422026077765376 & 0.97 & 269.72648 & -36.35458 & 17.9 & 1.021 & 0.432 & 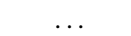 \\
\hline $355.1+02.3$ & Th 3-11 & 4058037456681232512 & 0.94 & 261.10970 & -31.72220 & 19.5 & 0.118 & 0.539 & $\ldots$ \\
\hline $355.2-02.0$ & PPA J1741-3405 & 4053444556158195712 & 0.43 & 265.49614 & -34.09294 & 20.0 & -1.024 & 0.636 & \\
\hline $355.2-02.5$ & H $1-29$ & 4041789389356118272 & 0.62 & 266.05776 & -34.29249 & 17.0 & -1.441 & 0.193 & $12.79_{-552}^{+2.69}$ \\
\hline $355.2-03.1$ & SP J1746-3 & 4041711392643900672 & 0.68 & 266.70100 & -34.60082 & 14.9 & 0.121 & 0.086 & $\ldots$ \\
\hline $355.3+03.1$ & PPA J1721-3110 & 4058127852814158208 & 0.61 & 260.41208 & -31.17627 & 18.2 & -0.099 & 0.164 & $\ldots$ \\
\hline $355.3+03.8$ & MPA J1719-3043 & 4058948024160146176 & 1.00 & 259.83362 & -30.72787 & 15.5 & 0.639 & 0.040 & \\
\hline $355.3-03.2$ & PPA J1747-3435 & 4041711044735017856 & 0.92 & 266.78472 & -34.59532 & 18.8 & -0.789 & 0.229 & $9.14_{-1.93}^{+2.04}$ \\
\hline $355.3-07.5$ & SB 42 & 4038618088434289664 & 1.00 & 271.46903 & -36.76014 & 18.1 & 0.032 & 0.153 & $\ldots$ \\
\hline $355.3-12.9$ & PaKn 1 & 6726848525705542016 & 1.00 & 277.52031 & -39.16982 & 18.0 & 0.188 & 0.135 & $\ldots$ \\
\hline $355.4-02.4$ & M 3-14 & 4041797566848828288 & 0.75 & 266.08599 & -34.11134 & 18.9 & & & \\
\hline $355.4-04.0$ & Hf $2-1$ & 4041465647722562688 & 0.81 & 267.80074 & -34.92311 & 17.4 & 0.073 & 0.249 & $4.95_{-1.25}^{+1.32}$ \\
\hline $355.4-04.3$ & K 5-34 & 4040802611953843584 & 0.81 & 268.09613 & -35.07160 & 19.3 & -0.416 & 0.637 & \\
\hline $355.4-05.7$ & MPA J1757-3547 & 4040485952573587456 & 0.21 & 269.49820 & -35.79939 & 20.0 & 4.572 & 1.130 & $\ldots$ \\
\hline $355.5+03.6$ & PHR J1720-3041 & 4058951288267947136 & 0.23 & 260.12618 & -30.69615 & 19.2 & 0.256 & 0.442 & $\ldots$ \\
\hline $355.5-02.8$ & MPA J1746-3412 & 4041758053185606656 & 0.96 & 266.57697 & -34.21034 & 15.5 & 0.097 & 0.046 & $\ldots$ \\
\hline $355.5-03.7$ & PHR J1749-3438 & 4041487500447313152 & 0.31 & 267.49522 & -34.64403 & 19.8 & 0.087 & 2.364 & \\
\hline $355.6+01.4$ & PPA J1729-3152 & 4057890740553838848 & 0.85 & 262.29583 & -31.87920 & 21.2 & & & . \\
\hline $355.6+05.1$ & MPA J1714-2946 & 4107290561090116224 & 0.95 & 258.70138 & -29.77974 & 17.5 & -0.217 & 0.133 & \\
\hline $355.6-02.3$ & PHR J1744-3355 & 4041804473208281088 & 0.35 & 266.11632 & -33.92221 & 16.6 & 0.258 & 0.139 & $4.20^{+1.37}$ \\
\hline $355.6-02.7$ & H 1-32 & 4041808145324509312 & 0.73 & 266.52637 & -34.06276 & 16.1 & & & $\ldots$ \\
\hline $355.6-02.9$ & SP J1746-3413 & 4041755579283791744 & 0.99 & 266.70024 & -34.22842 & 19.7 & 0.211 & 0.691 & $\ldots$ \\
\hline $355.7-03.0$ & H 1-33 & 4041751181235246720 & 0.96 & 266.95591 & -34.13475 & 18.3 & & . & $\ldots$ \\
\hline $355.7-03.4$ & H 2-23 & 4041553853231641728 & 0.93 & 267.24171 & -34.36479 & 18.5 & & & $\ldots$ \\
\hline $355.7-03.5$ & H 1-35 & 4041552891219095552 & 0.95 & 267.30802 & -34.38135 & 14.5 & 0.318 & 0.186 & $\ldots$ \\
\hline $355.8+04.5$ & PHR J1717-2954 & 4059257536673042944 & 0.98 & 259.43341 & -29.90828 & 19.4 & 0.183 & 0.441 & $\ldots$ \\
\hline $355.8-08.7$ & SB 43 & 4037722639347789056 & 0.75 & 273.09832 & -36.88063 & 20.5 & & 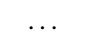 & \\
\hline $355.9+00.7$ & PPA J1732-3201 & 4054847803471988608 & 0.94 & 263.18535 & -32.01875 & 18.7 & $\cdots$ & $\ldots$ & $\ldots$ \\
\hline $355.9+02.7$ & Th 3-10 & 4058241003761354112 & 0.95 & 261.17023 & -30.86642 & 20.5 & & & \\
\hline $355.9+03.6$ & H $1-9$ & 4058987022459300096 & 0.70 & 260.38293 & -30.34686 & 14.8 & 0.012 & 0.231 & $6.02_{-1.66}^{+1.77}$ \\
\hline $355.9+04.1$ & PHR J1719-3003 & 4059196170135477120 & 0.23 & 259.94674 & -30.06534 & 20.7 & & & $\ldots$ \\
\hline $355.9+06.1$ & Terz N 7 & 4107592338352252544 & 0.98 & 257.99778 & -28.85951 & 17.7 & -0.121 & 0.124 & $\ldots$ \\
\hline $355.9-01.4$ & PN RPZM 33 & 4054095707502385408 & 0.26 & 265.36772 & -33.17268 & 18.3 & & & $\ldots$ \\
\hline $355.9-02.3$ & RPZM 37 & 4053847832044717824 & 0.98 & 266.28642 & -33.60223 & 18.9 & -1.206 & 0.452 & \\
\hline $355.9-04.2$ & M $1-30$ & 4040819138994544384 & 0.97 & 268.24558 & -34.63969 & 16.3 & 0.197 & 0.080 & $5.96_{-1.51}^{+1.83}$ \\
\hline $355.9-04.4$ & PHR J17 & 4040837487046953344 & 0.41 & 268.41591 & -34.72778 & 17.7 & 0.493 & 0.116 & ${ }^{-1.51}$ \\
\hline $356.0+02.8$ & PN PBOZ 3 & 4058250212203025792 & 0.96 & 261.24316 & -30.71783 & 20.1 &  & $\ldots$ & $\ldots$ \\
\hline $356.0-01.8$ & PPA J1743-3315 & 4053915250171942016 & 0.31 & 265.77975 & -33.26505 & 20.6 & & & \\
\hline $356.0-04.2$ & PHR J1753-3428 & 4041594668305141888 & 0.94 & 268.27046 & -34.47757 & 18.6 & 0.312 & 0.196 & $7.65_{-2.34}^{+2.37}$ \\
\hline $356.0-07.4 \mathrm{a}$ & SB 44 & 4038767175336290560 & 0.98 & 271.78264 & -36.04588 & 18.9 & & & $\ldots$ \\
\hline $356.0-07.4 \mathrm{~b}$ & SB 45 & 4038672754819227264 & 1.00 & 271.71884 & -36.11127 & 17.4 & -0.017 & 0.101 & $\ldots$ \\
\hline $356.1+02.7$ & Th 3-13 & 4058250070404444416 & 0.65 & 261.33070 & -30.67836 & 17.5 & $\ldots$ & $\ldots$ & $\ldots$ \\
\hline $356.1-02.1$ & PHR J1744-3319 & 4053868039851669760 & 0.97 & 266.21574 & -33.32498 & 21.0 & & & \\
\hline $356.1-03.3$ & H 2-26 & 4041661463727363072 & 0.24 & 267.46165 & -34.00846 & 17.4 & -0.533 & 0.123 & $15.60_{-3.35}^{+3.59}$ \\
\hline $356.1-03.4$ & $\begin{array}{l}\text { CTIOJ175006.71- } \\
340258.6\end{array}$ & 4041660334055294976 & 0.98 & 267.52792 & -34.04961 & 17.5 & 0.089 & 0.094 & $\cdots$ \\
\hline $356.1-08.6$ & SB 46 & 4037941579558523776 & 1.00 & 273.16613 & -36.53041 & 18.2 & -0.010 & 0.195 & $\ldots$ \\
\hline $356.2-00.3$ & Th 3-34 & 4054958446132297600 & 0.99 & 264.43704 & -32.25830 & 19.6 & $\ldots$ & $\ldots$ & $\ldots$ \\
\hline $356.2-03.2$ & PHR J1749-3347 & 4041855806630398208 & 0.53 & 267.34929 & -33.79483 & 18.2 & & & $\ldots$ \\
\hline $356.2-03.6$ & PPA J1751-3401 & 4041669950493674240 & 0.67 & 267.77879 & -34.02818 & 20.4 & & & \\
\hline $356.2-05.1$ & K 5-37 & 4042264240781873792 & 0.75 & 269.31518 & -34.79289 & 16.8 & 0.092 & 0.075 & $\ldots$ \\
\hline $356.2-08.9$ & PHR J1814-3635 & 4037753460036201088 & 1.00 & 273.55668 & -36.59319 & 15.1 & 0.089 & 0.046 & \\
\hline $356.4+03.7$ & K 5-31 & 4059107728241232768 & 0.29 & 260.55377 & -29.87686 & 19.4 & $\ldots$ & $\ldots$ & $\ldots$ \\
\hline $356.4-04.0$ & $\begin{array}{l}\text { CTIOJ175323.14- } \\
340653.7\end{array}$ & 4041622263451048064 & 0.24 & 268.34627 & -34.11487 & 20.0 & & & . \\
\hline $356.4-06.8$ & SB 48 & 4039031333003460736 & 1.00 & 271.31002 & -35.46883 & 17.5 & 0.053 & 0.206 & $\ldots$ \\
\hline
\end{tabular}


Table A.1: continued.

\begin{tabular}{|c|c|c|c|c|c|c|c|c|c|}
\hline PN G & PN name & Gaia source ID & reliability & $\begin{array}{c}\mathrm{RA} \\
(\mathrm{deg})\end{array}$ & $\begin{array}{c}\text { Dec } \\
(\mathrm{deg})\end{array}$ & $\begin{array}{c}\mathrm{G} \\
(\mathrm{mag})\end{array}$ & $\begin{array}{c}\omega \\
(\mathrm{mas})\end{array}$ & $\begin{array}{c}\sigma_{\omega} \\
(\mathrm{mas})\end{array}$ & $\begin{array}{l}\text { distance } \\
(\mathrm{kpc})\end{array}$ \\
\hline $356.5+01.5$ & Th 3-55 & 4058329239618656896 & 0.86 & 262.74524 & -31.01829 & 18.6 & 0.252 & 0.234 & $\ldots$ \\
\hline $356.5+19.1$ & $\mathrm{~Pa} 43$ & 6052674037595549440 & 1.00 & 247.10258 & -20.38565 & 18.9 & 0.281 & 0.226 & \\
\hline $356.5-01.8$ & PPA J1744-3252 & 4054021662258469248 & 0.54 & 266.11670 & -32.86994 & 19.9 & 0.378 & 0.504 & \\
\hline $356.5-02.3$ & M 1-27 & 4053955824662571648 & 0.69 & 266.68948 & -33.14310 & 13.9 & 0.270 & 0.022 & $3.23_{-0.22}^{+0.25}$ \\
\hline $356.5-03.4$ & MPA J1751-3339 & 4041696613843327488 & 0.39 & 267.83645 & -33.65358 & 20.9 & & & \\
\hline $356.5-03.6$ & H 2-27 & 4041691086085898112 & 0.27 & 267.96069 & -33.79345 & 18.2 & 0.186 & 0.199 & $8.70_{-264}^{+2.71}$ \\
\hline $356.5-03.9$ & H 1-39 & 4043128594411861120 & 0.95 & 268.33758 & -33.93293 & 15.5 & 0.031 & 0.045 & $10.88_{-2.29}^{+2.64}$ \\
\hline $356.5-04.1$ & PPA J1754-3358 & 4043113059511396480 & 0.92 & 268.51219 & -33.98112 & 17.8 & 0.250 & 0.115 & \\
\hline $356.6+05.9$ & Kn 124 & 4107710952444522496 & 0.93 & 258.59338 & -28.45761 & 17.4 & 0.280 & 0.091 & $\ldots$ \\
\hline $356.6+07.5$ & K 6-20 & 4108539434533747840 & 0.95 & 257.18728 & -27.51743 & 18.0 & 1.198 & 0.368 & $\ldots$ \\
\hline $356.6-04.7$ & PHR J1756-3414 & 4042344019826411520 & 0.24 & 269.20123 & -34.24223 & 19.7 & & & $\ldots$ \\
\hline $356.6-07.8$ & H 1-57 & 4038787447691293696 & 0.74 & 272.45529 & -35.73686 & 20.9 & & & $\ldots$ \\
\hline $356.7+05.4$ & Kn 125 & 4107508367466356224 & 0.36 & 259.15387 & -28.63996 & 15.5 & 0.108 & 0.036 & \\
\hline $356.7-02.6$ & MGE 356.7274-02.6316 & 4041970220238068608 & 0.34 & 267.05305 & -33.09772 & 19.8 & -0.340 & 0.523 & $\ldots$ \\
\hline $356.7-04.7$ & MPA J1757-3410 & 4042368797554199936 & 0.98 & 269.25233 & -34.17806 & 18.8 & -0.351 & 0.313 & \\
\hline $356.7-04.8$ & H 1-41 & 4042368312133298816 & 0.91 & 269.32961 & -34.16359 & 16.3 & 0.262 & 0.088 & $4.75_{-114}^{+1.40}$ \\
\hline $356.7-06.4$ & H $1-51$ & 4039109982533108736 & 0.62 & 271.12207 & -34.96680 & 20.1 & & & \\
\hline $356.8+03.3$ & Th 3-12 & 4059078758577969792 & 0.49 & 261.27551 & -29.75474 & 17.3 & 0.423 & 0.464 & $16.11_{-4.98}^{+4.95}$ \\
\hline $356.8-03.0$ & K 5-20 & 4041908991157987072 & 0.94 & 267.54481 & -33.23837 & 18.4 & 0.366 & 0.248 & $\ldots$ \\
\hline $356.8-03.6$ & PHR J1752-3330 & 4043198031104721664 & 0.97 & 268.12174 & -33.50121 & 14.8 & 0.039 & 0.032 & \\
\hline $356.8-05.4$ & H 2-35 & 4042115119631704064 & 0.23 & 270.07608 & -34.46121 & 20.6 & & & \\
\hline $356.8-11.7$ & Lo 17 & 6727251191784579072 & 0.98 & 276.96217 & -37.26527 & 18.3 & 0.283 & 0.157 & $2.89_{-0.55}^{+0.61}$ \\
\hline $356.9+00.9$ & PPA J1734-3102 & 4055336089712420480 & 0.85 & 263.64321 & -31.03548 & 20.5 & .. & $\ldots$ & $\ldots$ \\
\hline $356.9+02.2$ & MPA J1729-3016 & 4058603017938390528 & 0.37 & 262.34649 & -30.28002 & 19.8 & & 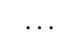 & \\
\hline $356.9+04.4$ & M 3-38 & 4059407207673857024 & 0.66 & 260.26862 & -29.04992 & 16.9 & 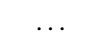 & $\ldots$ & $\ldots$ \\
\hline $356.9+04.5$ & M 2-11 & 4059405489712017280 & 0.72 & 260.13854 & -29.01087 & 17.4 & $\cdots$ & $\ldots$ & $\cdots$ \\
\hline $357.0+00.5$ & $\begin{array}{l}\text { SSTGLMC } \\
\text { G357.0758+00.5440 }\end{array}$ & 4055278919404314624 & 0.50 & 264.08282 & -31.12861 & 20.8 & $\cdots$ & $\cdots$ & $\cdots$ \\
\hline $357.0-04.4$ & PHR J1756-3342 & 4042399682594893056 & 0.25 & 269.16512 & -33.70913 & 18.4 & 0.655 & 0.225 & $6.30_{-2.85}^{+2.67}$ \\
\hline $357.1+03.6$ & M 3-7 & 4059510458668468992 & 0.84 & 261.14353 & -29.40550 & 16.2 & 0.172 & 0.054 & $5.25_{-0.94}^{+1.15}$ \\
\hline $357.1+04.4$ & Terz N 18 & 4059409887739353472 & 0.93 & 260.40827 & -28.92073 & 20.5 & & & \\
\hline $357.1-04.7$ & H 1-43 & 4042380857826322176 & 0.96 & 269.56016 & -33.79381 & 15.0 & 0.571 & 0.061 & $1.68_{-0.16}^{+0.20}$ \\
\hline $357.1-06.1$ & M 3-50 & 4042158138029882752 & 0.72 & 271.02171 & -34.47694 & 20.7 & & & -0.1 \\
\hline $357.2+01.4$ & $\mathrm{Al} 2-\mathrm{H}$ & 4058374899352518784 & 0.80 & 263.32094 & -30.44148 & 19.9 & 3.109 & 0.649 & $\ldots$ \\
\hline $357.2+07.2$ & Terz N 5 & 4108636668226400384 & 0.54 & 257.81191 & -27.19207 & 20.0 & -3.609 & 0.887 & \\
\hline $357.2+07.4$ & M 4-3 & 4108648286156728192 & 0.84 & 257.67406 & -27.14560 & 17.1 & 0.361 & 0.302 & $29_{-2.82}^{+2.80}$ \\
\hline $357.2-04.5$ & H 1-42 & 4042401439317575168 & 0.86 & 269.35488 & -33.59532 & 16.5 & & & $\ldots$ \\
\hline $357.2-09.8$ & SB 49 & 4038191134317113856 & 0.97 & 275.03853 & -36.12261 & 16.9 & 0.416 & 0.127 & . \\
\hline $357.3+02.3$ & K 6-25 & 4058647238955402112 & 0.97 & 262.47244 & -29.95287 & 20.1 & 2.660 & 1.538 & 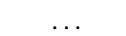 \\
\hline $357.3+02.8$ & PHR J1728-2936 & 4058717053628807424 & 0.84 & 262.03717 & -29.61497 & 20.2 & 0.556 & 1.141 & \\
\hline $357.3+03.3$ & M 3-41 & 4059513619827242496 & 0.94 & 261.49916 & -29.36394 & 15.6 & -1.932 & 0.568 & $6.47_{-1.56}^{+1.63}$ \\
\hline $357.3+04.0$ & H 2-7 & 4059722355127230080 & 0.98 & 260.85388 & -28.98501 & 18.7 & 0.007 & 0.254 & $7.46_{-2.14}^{+2.25}$ \\
\hline $357.3+05.7$ & PHR J17 & 4107751084656945408 & 0.94 & 259.25667 & -27.99151 & 20.0 & 0.037 & 0.715 & $\ldots$ \\
\hline $357.3-02.0$ & PPA J1747-3215 & 4055565445282016512 & 0.79 & 266.86885 & -32.26290 & 19.4 & 0.783 & 0.396 & $\ldots$ \\
\hline $357.3-06.5$ & SB 50 & 4039144990809866880 & 0.97 & 271.53428 & -34.55831 & 18.9 & -0.018 & 0.329 & $\ldots$ \\
\hline $357.4+22.8$ & $\mathrm{KnPa} 2$ & 6246462897568952192 & 1.00 & 244.65458 & -17.40417 & 16.8 & 0.183 & 0.092 & $\ldots$ \\
\hline $357.4-03.1$ & PHR J1752-3244 & 4043447104964040064 & 0.68 & 268.00216 & -32.73572 & 20.6 & & $\ldots$ & 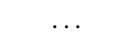 \\
\hline $357.4-03.2$ & M 2-16 & 4043444738331539200 & 0.69 & 268.14321 & -32.76412 & 18.4 & & & \\
\hline $357.4-03.5$ & M 2-18 & 4043426149764410240 & 0.99 & 268.40768 & -32.97999 & 16.7 & 0.067 & 0.190 & $9.80_{-269}^{+2.77}$ \\
\hline $357.4-04.6$ & M 2-22 & 4042499910043679232 & 0.55 & 269.63572 & -33.47690 & 17.1 & -0.086 & 0.110 & $8.63_{-1.92}^{+2.08}$ \\
\hline $357.4-07.2$ & SB 51 & 4039268243468439168 & 0.38 & 272.31982 & -34.79715 & 18.9 & 0.042 & 0.265 & $8.79_{-2.36}^{+2.42}$ \\
\hline $357.5+03.1$ & Th 3-16 & 4059487265900104704 & 0.33 & 261.85150 & -29.35408 & 15.2 & 0.093 & 0.037 & $\ldots$ \\
\hline $357.5+04.5$ & PPA J1722-2831 & 4059811376916989952 & 0.66 & 260.54359 & -28.52617 & 17.1 & 0.469 & 0.088 & $\ldots$ \\
\hline $357.5-05.1$ & PPA J1800-3341 & 4042420440156985472 & 0.71 & 270.17961 & -33.69990 & 16.7 & 0.154 & 0.066 & $\ldots$ \\
\hline $357.5-05.5$ & PPA J1802-3350 & 4042248469749871488 & 0.28 & 270.59899 & -33.84689 & 18.7 & 0.171 & 0.218 & $\ldots$ \\
\hline $357.6-03.0$ & PHR J1752-3233 & 4043502875085890688 & 1.00 & 268.04917 & -32.55210 & 19.0 & 0.299 & 0.423 & \\
\hline $357.6-03.3$ & Н 2-29 & 4043451880845295744 & 0.88 & 268.32007 & -32.67739 & 19.1 & 0.953 & 0.447 & $5.32_{-2.94}^{+3.00}$ \\
\hline $357.6-06.5$ & PHR J1806-3416 & 4039537795681073664 & 0.82 & 271.69709 & -34.26776 & 20.6 & $\ldots$ & $\ldots$ & \\
\hline $357.7+01.4$ & PPA J1734-3004 & 4058480869169238272 & 0.55 & 263.69438 & -30.07248 & 21.0 & & & \\
\hline $357.7-04.8$ & BMP J1759-3321 & 4042513447762858880 & 1.00 & 269.93838 & -33.35352 & 18.1 & 1.137 & 0.137 & $0.90_{-0.10}^{+0.12}$ \\
\hline $357.8+04.9$ & PPA J1721-2805 & 4107887316685844736 & 0.80 & 260.38855 & -28.08899 & 19.0 & -0.059 & 0.337 & \\
\hline $357.9+01.7$ & PPA J1733-2945 & 4058548072417571584 & 0.34 & 263.40998 & -29.75853 & 20.0 & 1.076 & 0.910 & \\
\hline $357.9-03.8$ & H 2-30 & 4043347083815456384 & 0.90 & 269.05805 & -32.62284 & 18.7 & -2.383 & 0.341 & $8.30_{-1.66}^{+1.75}$ \\
\hline $357.9-05.1$ & M 1-34 & 4042471361358211584 & 0.53 & 270.34250 & -33.29547 & 20.3 & . & $\ldots$ & $\ldots^{-1.66}$ \\
\hline $358.0+05.5$ & Kn J171C & 4107975110191710208 & 0.27 & 259.89577 & -27.57920 & 20.8 & & & \\
\hline $358.0+09.3$ & Th 3-1 & 4112252931941023104 & 0.98 & 256.43583 & -25.41709 & 17.2 & 0.086 & 0.098 & $\cdots$ \\
\hline $358.0-02.4$ & PPA J1750-3152 & 4055650107657556736 & 0.46 & 267.70231 & -31.87393 & 19.2 & 0.636 & 0.441 & \\
\hline $358.0-02.7$ & PN Al 2-O & 4043622756199128064 & 0.25 & 267.93871 & -32.05112 & 16.0 & 0.152 & 0.063 & \\
\hline $358.0-04.6$ & Sa 3-107 & 4042539909060351104 & 0.80 & 269.97915 & -32.98663 & 16.7 & 0.030 & 0.074 & $\cdots$ \\
\hline $358.0-05.1$ & Pe 1-11 & 4042468784326847872 & 0.40 & 270.42840 & -33.25679 & 20.6 & & & \\
\hline $358.1+03.5$ & PNG358.10+03.51 & 4059663874932471552 & 0.57 & 261.86157 & -28.63864 & 17.5 & -0.020 & 0.202 & $\ldots$ \\
\hline
\end{tabular}


Table A.1: continued.

\begin{tabular}{|c|c|c|c|c|c|c|c|c|c|}
\hline PN G & PN name & Gaia source ID & reliability & $\begin{array}{c}\text { RA } \\
(\mathrm{deg})\end{array}$ & $\begin{array}{l}\text { Dec } \\
(\mathrm{deg})\end{array}$ & $\begin{array}{c}\mathrm{G} \\
(\mathrm{mag})\end{array}$ & $\begin{array}{c}\omega \\
(\mathrm{mas})\end{array}$ & $\begin{array}{c}\sigma_{\omega} \\
(\mathrm{mas})\end{array}$ & $\begin{array}{c}\text { distance } \\
(\mathrm{kpc})\end{array}$ \\
\hline $358.2+001.0$ & Kn J1737.3-2952 & 4059999638214891392 & 0.87 & 264.33052 & -29.87128 & 19.7 & 0.291 & 0.385 & 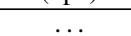 \\
\hline $358.2+01.4$ & $\begin{array}{l}\text { MGE } \\
358.2600+01.4563\end{array}$ & 4058565153580197760 & 0.26 & 263.93162 & -29.63954 & 18.9 & -0.362 & 0.303 & \\
\hline $358.2+03.5$ & H $2-10$ & 4059671537243938944 & 0.93 & 261.88692 & -28.51860 & 19.1 & -1.589 & 0.486 & $8.97_{-2.29}^{+2.39}$ \\
\hline $358.2+04.2$ & M 3-8 & 4059931331159092736 & 0.74 & 261.21707 & -28.09854 & 18.1 & 0.467 & 0.278 & $6.19^{2+.04}$ \\
\hline $358.3+03.0$ & H 1-17 & 4059627659823489024 & 0.92 & 262.41914 & -28.67287 & 16.7 & & & \\
\hline $358.3-07.3$ & SB 52 & 4039592182712537728 & 0.27 & 272.91656 & -34.00643 & 18.8 & -0.226 & 0.402 & \\
\hline $358.3-21.6$ & IC 1297 & 6714803311390242048 & 1.00 & 289.34769 & -39.61287 & 15.7 & 0.225 & 0.054 & $3.92_{-0.58}^{+0.71}$ \\
\hline $358.4+01.6$ & JaSt 3 & 4060084777353646976 & 0.23 & 263.84438 & -29.37158 & 19.7 & -0.264 & 0.505 & $7.20_{-23}^{+2.50}$ \\
\hline $358.4+01.7$ & JaSt 2 & 4060087530475429504 & 0.21 & 263.75379 & -29.37095 & 20.3 & 2.267 & 1.015 & $8.23_{-4.51}^{+4.12}$ \\
\hline $358.4+02.7$ & PHR J1731-2850 & 4058860024479453184 & 0.39 & 262.80953 & -28.84373 & 17.3 & 0.786 & 0.108 & $\ldots$ \\
\hline $358.4+03.3$ & Th $3-19$ & 4059684456483740800 & 0.75 & 262.17418 & -28.45548 & 18.4 & & & $\ldots$ \\
\hline $358.4-04.5$ & K 6-38 & 4043676185562267520 & 0.97 & 269.99596 & -32.59953 & 16.4 & 0.045 & 0.063 & - \\
\hline $358.4-05.5$ & PHR J1804-3306 & 4042860481114840832 & 0.64 & 271.06250 & -33.10841 & 20.0 & & & \\
\hline $358.5+02.6$ & M 3-57 & 4058868309558781952 & 0.99 & 262.94739 & -28.70072 & 18.3 & 0.217 & 0.218 & $1.99_{-035}^{+0.39}$ \\
\hline $358.5+02.9$ & $\mathrm{Al} 2-\mathrm{F}$ & 4059634527341796480 & 0.98 & 262.62682 & -28.59859 & 19.0 & 1.294 & 0.397 & $10.67_{-7.61}^{+5.08}$ \\
\hline $358.5-01.7$ & JaSt 64 & 4055921240421711744 & 0.94 & 267.23341 & -31.11189 & 20.0 & 1.559 & 0.888 & \\
\hline $358.5-02.5$ & PN M 4-7 & 4055661893097358208 & 0.35 & 267.93623 & -31.60027 & 18.9 & 0.143 & 0.242 & \\
\hline $358.5-04.2$ & H 1-46 & 4043695079072981248 & 0.94 & 269.76046 & -32.36209 & 15.1 & -0.447 & 0.242 & $7.85_{-1.82}^{+1.91}$ \\
\hline $358.5-07.3$ & NGC 6563 & 4039600536544395392 & 0.94 & 273.01075 & -33.86850 & 17.3 & 1.071 & 0.129 & $1.00_{-0.12}^{+0.16}$ \\
\hline $358.6+07.8$ & M 3-36 & 4109362831302119552 & 0.99 & 258.16317 & -25.72710 & 16.8 & -0.032 & 0.073 & $\ldots .1$ \\
\hline $358.6-01.1$ & JaSt 58 & 4056008823359403904 & 0.27 & 266.71736 & -30.62824 & 21.1 & & & \\
\hline $358.6-02.4$ & K 6-16 & 4055693053035857152 & 0.25 & 268.00152 & -31.29739 & 19.2 & 0.146 & 0.327 & \\
\hline $358.6-05.5$ & M 3-51 & 4042869590720165888 & 0.92 & 271.23428 & -32.90031 & 18.6 & 0.360 & 0.206 & $5.27_{-1.80}^{+1.96}$ \\
\hline $358.7+05.2$ & M 3-40 & 4108085748534671744 & 0.96 & 260.61787 & -27.14513 & 16.9 & 0.152 & 0.084 & \\
\hline $358.7-03.0$ & К 6-34 & 4043664881255111552 & 0.41 & 268.67153 & -31.52876 & 17.3 & 0.110 & 0.124 & $5.34_{-1.16}^{+1.28}$ \\
\hline $358.7-05.1$ & SB 53 & 4042933568622953344 & 0.33 & 270.86890 & -32.62374 & 19.8 & & & $\ldots$ \\
\hline $358.7-05.2$ & H 1-50 & 4042891061192838400 & 0.70 & 270.97295 & -32.69500 & 15.6 & & & $\ldots$ \\
\hline $358.7-08.8$ & $\mathrm{~Pa} 46$ & 4044593693643693312 & 1.00 & 274.79702 & -34.33849 & 18.7 & 0.107 & 0.189 & \\
\hline $358.8+01.2$ & $\begin{array}{l}\text { MGE } \\
358.8655+01.2862\end{array}$ & 4060102820566222080 & 0.56 & 264.46951 & -29.22020 & 21.3 & & (1) & $\ldots$ \\
\hline $358.8+01.7$ & JaSt 5 & 4060288397548604160 & 0.53 & 263.96786 & -28.97428 & 20.5 & -0.506 & 0.833 & $7.60_{-2.19}^{+2.22}$ \\
\hline $358.8+04.0$ & Th 3-15 & 405992232885 & 0.99 & 261.79441 & -27.73268 & 19.1 & 1.964 & 0.429 & $\ldots$ \\
\hline $358.8+04.1$ & SaWe 2 & 4059970363790649600 & 0.92 & 261.75150 & -27.67610 & 20.4 & & & - \\
\hline $358.8-06.0$ & MPA J1807-3254 & 4042759051082040960 & 0.58 & 271.84153 & -32.90860 & 16.5 & 0.009 & 0.080 & \\
\hline $358.9+03.2$ & H 1-20 & 4061195563412585344 & 0.98 & 262.68254 & -28.06857 & 20.0 & & $\ldots$ & $\ldots$ \\
\hline $358.9+03.4$ & H 1-19 & 4061211506326213760 & 0.68 & 262.51064 & -27.98836 & 18.4 & 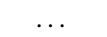 & $\ldots$ & $\ldots$ \\
\hline $358.9+04.7$ & PHR J1724-2716 & 4108036407947330176 & 0.67 & 261.22755 & -27.27306 & 20.3 & & & \\
\hline $358.9-00.7$ & M 1-26 & 4056786556015410816 & 0.98 & 266.49027 & -30.20024 & 12.6 & 0.423 & 0.029 & $2.26_{-0.14}^{+0.16}$ \\
\hline $358.9-01.5$ & JaSt 65 & 4055974360527366272 & 0.27 & 267.33339 & -30.60168 & 17.7 & -1.169 & 0.566 & $\ldots$ \\
\hline $358.9-01.9$ & MB 4403 & 4055764113306052608 & 0.94 & 267.71299 & -30.86853 & 19.2 & -0.752 & 0.471 & \\
\hline $358.9-02.1$ & PHR J1751-3059 & 4055764285137097728 & 0.93 & 267.91158 & -30.99831 & 20.7 & 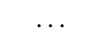 & & \\
\hline $358.9-03.7$ & H 1-44 & 4043963707852231808 & 1.00 & 269.54434 & -31.71557 & 18.6 & 0.481 & 0.270 & $8.50_{-2.94}^{+2.88}$ \\
\hline $359.0+02.8 \mathrm{a}$ & Kn 127 & 4061135949134377984 & 0.23 & 263.07691 & -28.26190 & 17.2 & 0.052 & 0.079 & $\ldots$ \\
\hline $359.0+03.9$ & Kn 126 & 4061424669711218304 & 0.87 & 262.02501 & -27.65479 & 17.3 & 0.004 & 0.090 & - \\
\hline $359.0+09.0$ & K 5-25 & 4112488399242497792 & 0.83 & 257.35735 & -24.72103 & 16.2 & 0.071 & 0.053 & - \\
\hline $359.0-04.1$ & M 3-48 & 4043780192529755520 & 0.27 & 269.98666 & -31.90795 & 19.9 & $\ldots$ & $\ldots$ & $\ldots$ \\
\hline $359.0-04.9$ & PHR J1803-3218 & 4042959407107886464 & 0.32 & 270.82107 & -32.30677 & 20.3 & & & \\
\hline $359.1+15.1$ & Abell 40 & 4127291600965496320 & 1.00 & 252.14368 & -21.01403 & 18.8 & -0.033 & 0.220 & $5.44_{-1.30}^{+1.39}$ \\
\hline $359.1-02.3$ & M 3-16 & 4056131006637615488 & 0.50 & 268.19186 & -30.82643 & 17.1 & 0.015 & 0.113 & $5.17_{-1.00}^{+1.10}$ \\
\hline $359.2+04.7$ & Th 3-14 & 4109555589418641408 & 0.65 & 261.43367 & -26.96334 & 15.3 & 0.064 & 0.037 & $11.45_{-2.70}^{+3.32}$ \\
\hline $359.2-03.1$ & PHR J1756-3112 & 4044074140098515840 & 0.84 & 269.12009 & -31.21073 & 19.8 & & & $\ldots$ \\
\hline $359.2-33.5$ & Hen 3-1863 & 6680314483486887552 & 1.00 & 304.86959 & -41.52434 & 10.7 & 0.336 & 0.040 & \\
\hline $359.3+03.6$ & $\mathrm{Al} 2-\mathrm{E}$ & 4061439208263407616 & 0.24 & 262.56015 & -27.50566 & 20.4 & .. & $\ldots$ & .. \\
\hline $359.3-00.9$ & $\mathrm{Hb} 5$ & 4056807274938145024 & 0.51 & 266.98401 & -29.99424 & 17.7 & & & \\
\hline $359.3-01.8$ & M 3-44 & 4056355822397882880 & 0.52 & 267.82884 & -30.39811 & 16.0 & 0.083 & 0.062 & $6.27_{-1.25}^{+1.51}$ \\
\hline $359.3-03.1$ & M 3-17 & 4044076167334983808 & 0.60 & 269.10688 & -31.07147 & 16.2 & 0.195 & 0.062 & $6.18_{-1.68}^{+2.49}$ \\
\hline $359.4-03.2 \mathrm{a}$ & $\begin{array}{l}\text { CTIOJ175709.24- } \\
310503.8\end{array}$ & 4044065099116926976 & 0.53 & 269.28859 & -31.08428 & 19.5 & 1.531 & 0.818 & \\
\hline $359.4-03.3$ & PHR J1757-3106 & 4044067061987628032 & 0.70 & 269.46543 & -31.10301 & 17.5 & -0.007 & 0.142 & \\
\hline $359.4-03.4$ & Н 2-33 & 4044019130131217408 & 0.78 & 269.55225 & -31.13511 & 18.8 & -0.894 & 0.407 & $8.28_{-244}^{+2.62}$ \\
\hline $359.4-05.6$ & BMP J1807-3215 & 4043004693207939584 & 0.37 & 271.77936 & -32.25613 & 17.6 & 0.422 & 0.118 & \\
\hline $359.4-06.3$ & PPA J1809-3233 & 4042752973769893504 & 0.94 & 272.47010 & -32.56233 & 17.1 & 0.130 & 0.079 & \\
\hline $359.4-08.5$ & SB 55 & 4044653651432842752 & 1.00 & 274.86079 & -33.61864 & 15.8 & 0.166 & 0.059 & $6.62_{-1.65}^{+2.13}$ \\
\hline $359.5-01.2$ & JaSt 66 & 4056429038638084736 & 0.99 & 267.34209 & -29.99097 & 20.3 & -0.272 & 0.945 & $8.42_{-2.62}^{-2.65}$ \\
\hline $359.6+02.2$ & $\mathrm{Al} 2-\mathrm{K}$ & 4060495277524149504 & 0.92 & 264.05911 & -28.01286 & 19.2 & 0.696 & 0.363 & \\
\hline $359.6-04.8$ & H 2-36 & 4043846678596007168 & 0.50 & 271.03227 & -31.65304 & 19.1 & 0.308 & 0.332 & $6.92_{-2.00}^{+2.02}$ \\
\hline $359.7+06.0$ & BMP J1721-2554 & 4109913205565555072 & 0.99 & 260.49193 & -25.90677 & 19.3 & 0.434 & 0.301 & \\
\hline $359.7-01.4$ & JaSt 73 & 4056423373608448000 & 0.44 & 267.69925 & -29.88721 & 17.8 & -0.127 & 0.137 & $22.64_{-7.18}^{+7.59}$ \\
\hline $359.7-01.7$ & RPZM 43 & 4056393407631404416 & 0.60 & 267.95338 & -30.04274 & 18.0 & 0.058 & 0.153 & \\
\hline $359.7-01.8$ & M 3-45 & 4056389975989346816 & 0.58 & 268.02484 & -30.08727 & 17.7 & 0.135 & 0.126 & $5.02_{-1.25}^{+1.47}$ \\
\hline
\end{tabular}


A\&A proofs: manuscript no. aanda

Table A.1: continued.

\begin{tabular}{|c|c|c|c|c|c|c|c|c|c|}
\hline PN G & PN name & Gaia source ID & reliability & $\begin{array}{c}\text { RA } \\
\text { (deg) }\end{array}$ & $\begin{array}{c}\text { Dec } \\
\text { (deg) }\end{array}$ & $\begin{array}{c}\mathrm{G} \\
(\mathrm{mag})\end{array}$ & $\begin{array}{c}\omega \\
(\mathrm{mas})\end{array}$ & $\begin{array}{c}\sigma_{\omega} \\
(\mathrm{mas})\end{array}$ & $\begin{array}{c}\text { distance } \\
(\mathrm{kpc})\end{array}$ \\
\hline $359.7-02.6$ & H $1-40$ & 4056120934862926848 & 0.56 & 268.90021 & -30.55902 & 15.9 & -1.344 & 0.326 & $9.82_{-2.68}^{+2.93}$ \\
\hline $359.7-04.4 \mathrm{a}$ & PPA J1802-3124 & 4043892067774459264 & 0.40 & 270.62622 & -31.40457 & 19.5 & -0.138 & 0.462 & $\ldots$ \\
\hline $359.8+02.4$ & Th 3-33 & 4060520527559308800 & 0.99 & 263.95053 & -27.72234 & 19.3 & $\ldots$ & $\ldots$ & $\ldots$ \\
\hline $359.8+03.5$ & PHR J1731-2709 & 4061545139294728448 & 0.84 & 262.94898 & -27.15521 & 20.8 & $\ldots$ & $\ldots$ & $\ldots$ \\
\hline $359.8+03.7$ & Th 3-25 & 4061467932992418304 & 0.47 & 262.69470 & -27.09997 & 18.1 & 0.172 & 0.220 & $8.00^{+2.53}$ \\
\hline $359.8+05.2$ & Terz N 19 & 4109665712365779968 & 0.86 & 261.34842 & -26.19806 & 19.0 & -1.284 & 0.353 & ${ }^{-2.45}$ \\
\hline $359.8+05.6$ & M 2-12 & 4109870908774509184 & 0.96 & 261.00612 & -25.98981 & 14.2 & 0.170 & 0.032 & $5.20_{-0.75}^{+1.02}$ \\
\hline $359.8-07.2$ & M 2-32 & 4045707288433971072 & 0.99 & 273.71084 & -32.61535 & 15.6 & -0.052 & 0.065 & $9.19^{+1.167}$ \\
\hline $359.9+05.1$ & M 3-9 & 4109665059557150592 & 1.00 & 261.43071 & -26.19874 & 17.7 & 0.653 & 0.131 & $1.93_{-0.44}^{+0.75}$ \\
\hline $359.9-01.8$ & MPA J1752-2953 & 4056399076974094208 & 0.60 & 268.20472 & -29.88356 & 19.2 & -0.710 & 0.701 & $\ldots$ \\
\hline $359.9-04.5$ & M 2-27 & 4043876228021661696 & 0.63 & 270.96905 & -31.29643 & 17.9 & $\ldots$ & $\ldots$ & $\ldots$ \\
\hline $359.9-05.4$ & KFL 9 & 4049041248260620800 & 0.90 & 271.83060 & -31.71563 & 18.0 & -0.294 & 0.275 & \\
\hline $359.9-07.4$ & SB 56 & 4045704990671786112 & 0.53 & 273.88528 & -32.63350 & 17.4 & 0.170 & 0.125 & $\cdots$ \\
\hline $\mathrm{J} 16$ & $\ldots$ & 4688984022393506432 & 1.00 & 12.90273 & -72.53528 & 20.4 & -0.454 & 0.628 & $\ldots$ \\
\hline
\end{tabular}

